University of Louisville

ThinkIR: The University of Louisville's Institutional Repository

Electronic Theses and Dissertations

$5-2011$

\title{
Fluid dynamic analysis of flow in orbiting dishes and the effects of flow on shear stress and endothelial cellular responses.
}

Amlan Chakraborty 1976-

University of Louisville

Follow this and additional works at: https://ir.library.louisville.edu/etd

\section{Recommended Citation}

Chakraborty, Amlan 1976-, "Fluid dynamic analysis of flow in orbiting dishes and the effects of flow on shear stress and endothelial cellular responses." (2011). Electronic Theses and Dissertations. Paper 230. https://doi.org/10.18297/etd/230

This Doctoral Dissertation is brought to you for free and open access by ThinkIR: The University of Louisville's Institutional Repository. It has been accepted for inclusion in Electronic Theses and Dissertations by an authorized administrator of ThinkIR: The University of Louisville's Institutional Repository. This title appears here courtesy of the author, who has retained all other copyrights. For more information, please contact thinkir@louisville.edu. 


\title{
FLUID DYNAMIC ANALYSIS OF FLOW IN ORBITING DISHES AND THE EFFECTS OF FLOW ON SHEAR STRESS AND ENDOTHELIAL CELLULAR RESPONSES
}

\author{
By \\ Amlan Chakraborty \\ B.S., Haldia Institute of Technology, India, 2000 \\ M.S., New Mexico State University, NM, U.S.A, 2006
}

A Dissertation Submitted to the Faculty of the Graduate School of the University of Louisville in Partial Fulfillment of the Requirements of the Degree of

\section{Doctor of Philosophy}

Department of Chemical Engineering

University of Louisville

Louisville, KY, U.S.A

May, 2011 
Copyright 2011 by Amlan Chakraborty

All rights reserved 


\title{
FLUID DYNAMIC ANALYSIS OF FLOW IN ORBITING DISHES AND THE EFFECTS OF FLOW ON SHEAR STRESS AND ENDOTHELIAL CELLULAR RESPONSES
}

\author{
By \\ Amlan Chakraborty \\ B.S., Haldia Institute of Technology, India, 2000 \\ M.S., New Mexico State University, NM, U.S.A, 2006 \\ A Dissertation Approved on \\ March 28, 2011
}

By the following Dissertation Committee:

R. Eric. Berson (Dissertation Director)

Bodduluri Haribabu

Moises Carreon

Venkatakrishna R. Jala

M. Keith Sharp

James Watters 


\section{DEDICATION}

This dissertation is dedicated to my beloved wife

Mrs. Debarchana Chakraborty

and my respected parents

Mrs. Atreyi Chakraborty

\& Mr. Sujit Chakraborty

This work is dedicated to all the people who have supported me by any form which helps me to complete this work successfully. I am especially thankful to my wife Debarchana for providing me continuous morale support. Unless getting that unforgettable assistance, the project couldn't be completed successfully. 


\section{ACKNOWLEDGEMENT}

I especially and sincerely acknowledge my research advisor Dr. R. Eric Berson for his expert guidance, advice in every step of this effort. This work couldn't be completed successfully without his sincere assistance and motivation. Further, I would like to express my sincere acknowledgment and appreciation to Prof. Haribabu Bodduluri for providing me his invaluable technical suggestions and all of his laboratory facilities to continue my research in the last four years. I sincerely acknowledge Dr. M. Keith Sharp for his immense technical support in the field of fluid dynamics. I must say that this effort couldn't be completed successfully unless his expert guidance. I am especially thankful to Dr. V.R. Jala for his continuous help and support for me in obtaining the knowledge and research techniques in advance bio-medical research. Further, I'd like to thank my other committee members, Dr. James Watters and Dr. Moises Carreon for their comments and assistance over the past years.

Last but not least I am thankful to my entire colleague especially Jonathan Thomas and Zhuoliang Ye, all of my lab members especially Dr. Steven Mathis, Ms. Sobha Bodduluri, Dr. Suchismita Satpathy, and Ms. Michelle Smith for their continuous support and assistance in the way of this effort over the past years. 


\title{
ABSTRACT \\ FLUID DYNAMIC ANALYSIS OF FLOW IN ORBITING DISHES AND THE EFFECTS OF FLOW ON SHEAR STRESS AND ENDOTHELIAL CELLULAR RESPONSES
}

\author{
Amlan Chakraborty
}

March 28, 2011

This work presents a novel comprehensive effort to understand the fluid dynamics within orbiting dishes and the effects of the resulting oscillating fluid flow on shear and endothelial cellular responses within the dishes. It is well documented that hemodynamic parameters, especially wall shear stress (WSS), have been shown to play important roles in altering various endothelial cellular responses, intracellular pathways and gene expression, and to have significant impacts on disease progression, such as atherosclerotic plaque development. In practice, wall shear stress (WSS) is oscillatory rather than steady due to the travelling waveform and varies across the surface of the dish at any instant in time. The first part of this effort presents a computational model which provides complete spatial and temporal resolution of oscillatory WSS over the bottom surface of an orbiting Petri dish throughout the orbital cycle. The model was reasonably well validated by the analytical solution and the results were compared to the tangential WSS magnitudes obtained using one-dimensional optical velocimetry at discreet locations on the bottom of an orbiting dish. 
A thorough fluid dynamic analysis was performed in the next part of this work to understand the fluid motion inside the dish by investigating the system properties that affect WSS. To identify the effects of each of those properties on WSS, a dimensional analysis study was performed which includes analyses of three dimensionless parameters - Slope ratio, Froude Number, and Stokes Number. A fourth Reynolds Number was held constant. By analyzing a range from low to high values for each of the parameters, transition points for each of the flow parameters were determined. Further the nature of WSS at different radii $(20 \%, 40 \%, 60 \%$ and $80 \%$ of the maximum radius of the dish) on the bottom surface was studied as a function of combinations of various dimensionless parameters.

In the last part of this study, this model was applied to understand the effects of oscillatory WSS on endothelial cellular responses, including cell proliferation, morphology, and atherogenic gene expression. Since WSS on the bottom of the dish is two-dimensional, a new directional oscillatory shear index (DOSI) was developed to quantify the directionality of oscillating shear. DOSI approached zero for bidirectional oscillatory shear of equal magnitudes near the center and approached one for unidirectional oscillatory shear near the wall, where large tangential WSS dominated a much smaller radial component. Cellular responses including cell proliferation, area, shape index, orientation, and atherogenic gene expressions at mRNA level (I-CAMl, ESelectin, IL-6) were then correlated with different DOSI level under the same flow conditions. A comprehensive statistical analysis demonstrated that DOSI significantly affects all the responses, indicating that, in addition to shear magnitudes, directionality and the oscillatory nature of shear significantly influence cellular responses. 


\section{TABLE OF CONTENTS}

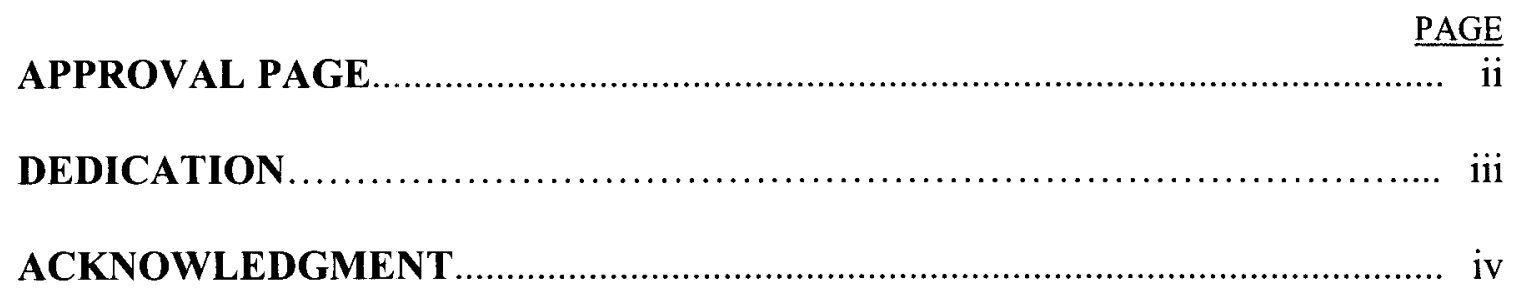

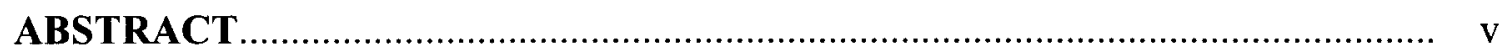

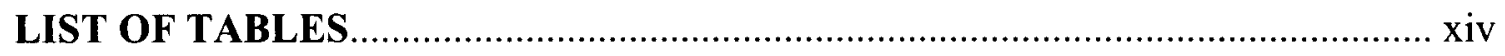

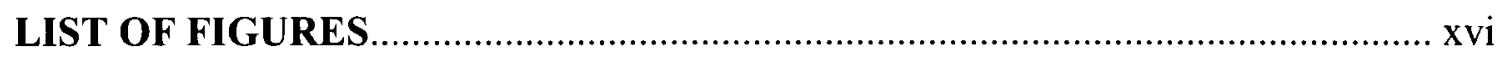

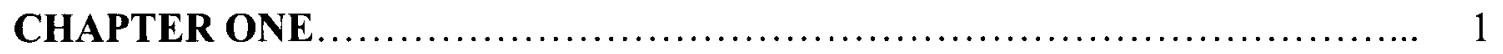

Introduction........................................................................ 1

Objectives................................................ 4

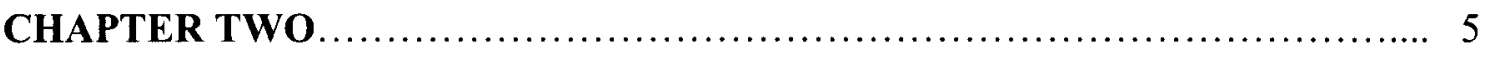

Generation of spatial and temporal resolution of oscillatory fluidic shear in orbiting dishes by CFD and comparison with experimentally and analytically determined

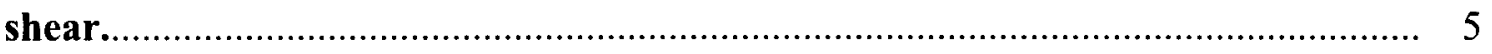

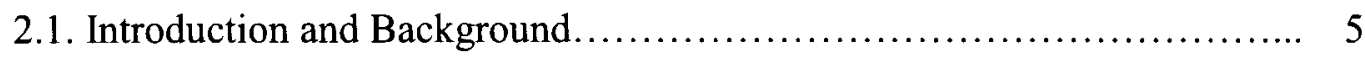

2.1.1. Definition of Shear stress.............................. 5

2.1.2. Experimental methods to determine fluidic shear stress........ 6

2.1.3. Advantages of using orbital shakers for generating shear........ 12

2.1.4. Computational Fluid Dynamics (CFD)..................... 13

2.1.5. Methodology of obtaining a CFD solution for a particular system of 
interest

2.1.6. Basic equations that control fluid flow in CFD................... 20

2.1.7. Methods of discritization of governing partial differential

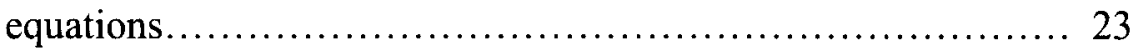

2.1.8. Finite Difference Method (FDM) ........................... 24

2.1.9. CFD techniques in analyzing multiphase flow.............. 29

2.1.9.1. The Euler-Lagrange approach...................... 29

2.1.9.2. The Euler-Euler approach.................... 30

2.1.10. Application of CFD in quantifying wall shear stress... 31

2.2. Materials and Methods........................................ 37

2.2.1. Modeling and computation........................... 37

2.2.2. Case set up procedure.................................... 39

2.3. Results and discussion...................................... 40

2.3.1. Sample simulation result............................ 40

2.3.2. WSS as a function of radius and orbital time............... 41

2.3.3. WSS as a function of orbital velocity ................... 43

2.3.4. Comparison with analytical solution..................... 45

2.3.5. Comparison with experimental oscillatory WSS............. 46

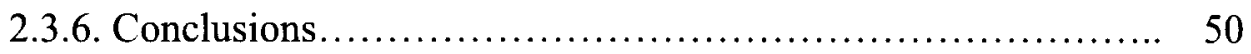

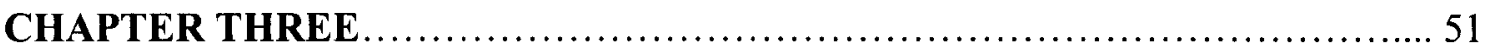

Dimensional analysis of flow in orbiting Petri-dishes $\ldots \ldots \ldots \ldots \ldots \ldots \ldots \ldots \ldots \ldots \ldots$

3.1. Introduction and Background ................................ 51

3.1.1. Brief description about the dimensionless parameters.............. 51 


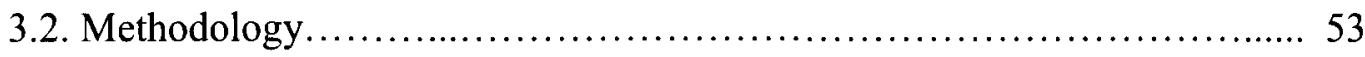

3.2.1. Development of dimensionless parameters........................ 53

3.2.2. Free surface analysis of dimensional parameters in determining

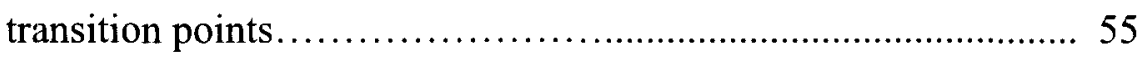

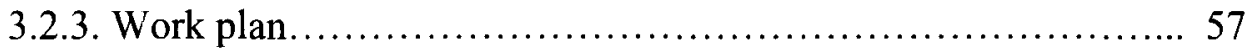

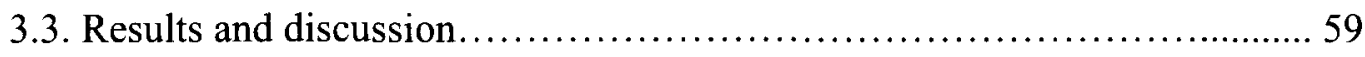

3.3.1. WSS analysis as a function of Slope ratio at constant Stokes

Number and Froude Number......................................................... 59

3.3.2. WSS analysis as a function of Froude Number at constant Stokes

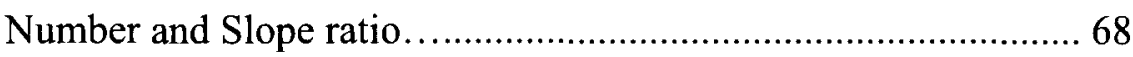

3.3.3. WSS analysis as a function of Stokes Number at constant Froude

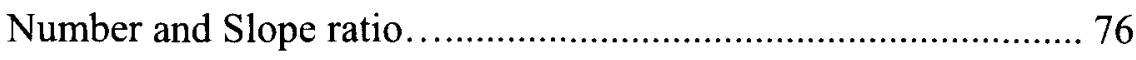

3.3.4. Conclusions...................................................... 84

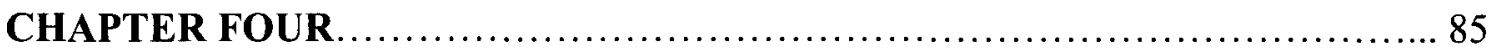

Directional oscillatory shear index (DOSI) and the effects of DOSI and shear magnitudes on endothelial cell proliferation and morphology ................... 85

4.1. Introduction and Background ..................................... 85

4.1.1. Effects of steady unidirectional shear....................... 85

4.1.2. Effects of unidirectional oscillatory shear stress on cellular responses........................................... 92

4.1.3. Unidirectional pulsatile shear effects........................ 94

4.2. Materials and Methods......................................... 97

4.2.1. CFD methodology and case set up procedure.................. 97 
4.2.2. Experimental protocol..................................... 98

4.2.3. Microscopy and image analysis.............................. 99

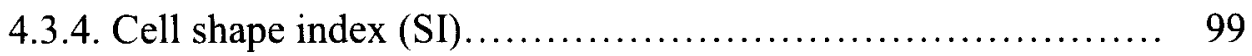

4.2.5. Directional oscillatory shear index (DOSI)................... 100

4.2.6. Statistical methods.................................... 101

4.2.6.1. Poisson distribution.............................. 101

4.2.6.2. Gaussian distribution........................... 103

4.2.6.3. Gamma distribution.............................. 104

4.2.6.4. Circular linear regression....................... 104

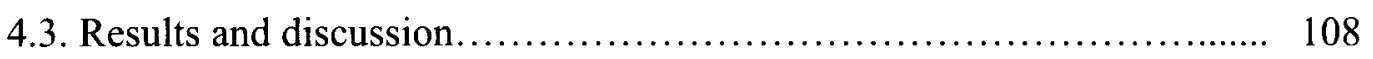

4.3.1. Determination of wall shear stress by CFD.................. 108

4.3.2. Determination of directional oscillatory shear index (DOSI) .... 112

4.3.3. Effects of oscillatory shear on cell proliferation, area and shape

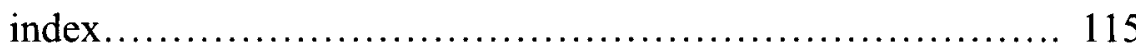

4.3.4. Individual and interacting effects of DOSI, shear magnitude, and orbital speed on cell proliferation............................ 118

4.3.5. Individual and interacting effects of DOSI, shear magnitude, and orbital speed on cell area............................................ 118

4.3.6. Individual and interacting effects of DOSI, shear magnitude, and orbital speed on cell shape index............................... 119

4.3.7. Effects of DOSI, shear magnitude, and orbital speed on cell orientation

4.3.8. Conclusions. 
Effects of DOSI and shear magnitudes of oscillating shear on cell proliferation, morphology, and atherogenic gene expressions at the mRNA level in the presence of

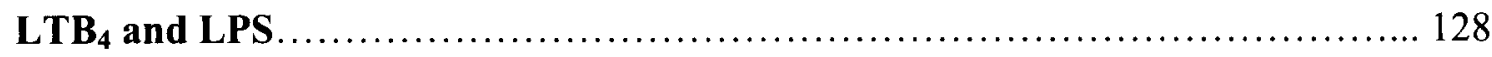

5.1. Introduction and Background...................................... 128

5.1.1. Mechanism of endothelial cellular responses to fluidic shear and effects of hemodynamic parameters on vascular inflammation and atherosclerosis.

5.1.2. Effects of fluid shear stress on various intracellular signaling molecules and gene expressions 129

5.1.3. Inflammation and atherosclerosis.

5.1.4. The role of fluid shear stress on atherosclerosis 133

5.1.5. Role of Leukotrienes and its receptors in atherosclerosis

5.1.6. Importance of bi-directional oscillatory WSS in atherosclerosis plaque formation.

5.2. Materials and Methods. 140

5.2.1. Multiphase CFD model and simulation process. 140

5.2.2. Cell culture and shear stress application. 140

5.2.3. Quantitative Real time polymerase chain reaction (RT-PCR)..... 141

5.2.4. Microscopy and image analysis.

5.2.5. Statistical analysis. 142

5.2.5.1. ANOVA analysis to understand the effects of DOSI and shear magnitude on gene expressions 
5.2.5.2. ANOVA analysis to understand the effects of DOSI and shear magnitude on cell proliferation and morphology. 143

5.3. Results and discussion 144

5.3.1. Determination of WSS by CFD and estimation of DOSI. 144

5.3.2. Effects of DOSI and shear magnitude on cell proliferation and cell area in the presence of $\mathrm{LTB}_{4}$ and LPS

5.3.3. Individual and interacting effects of DOSI and shear magnitude on cell proliferation and cell area in the presence of $\mathrm{LTB}_{4}$ and LPS..151

5.3.4. Effects of DOSI and shear magnitude on aherogenic gene expressions in the presence of $\mathrm{LTB}_{4}$ and LPS

5.3.5. Individual and interacting effects of DOSI and shear magnitude on Gene expressions in the presence of $\mathrm{LTB}_{4}$ and LPS

5.3.6. Conclusions 157

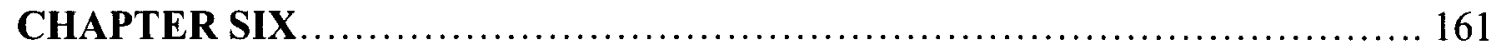

CONCLUSION \& FUTURE RESEARCH............................... 161

6.1. Conclusion......................................................... 161

6.2. Future recommendations...................................... 162

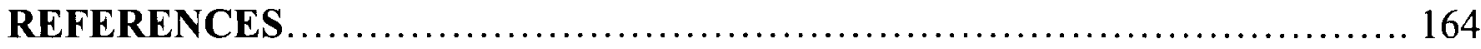

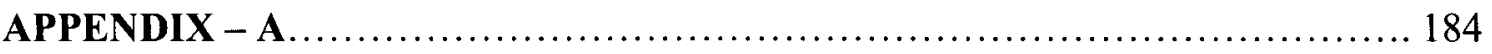

Computer programs for FLUENT simulations ........................ 184

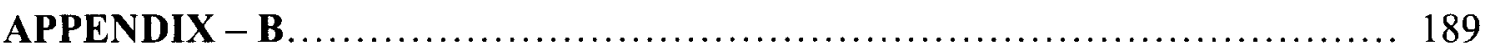

Radial and tangential WSS data for different combinations of dimensionless

Parameters 
Relative gene expression data for atherogenic genes in HUVECs under shear exposure at $100 \mathrm{rpm}$ at the mRNA level, obtained by real time PCR analysis.. 210

APPENDIX - D. 212

Table D1. System properties and dimensionless numbers for Figure 2.10_... 212

Table D2. System properties and related dimensionless numbers corresponding to DOSI analysis cases in Chapter Four. 212

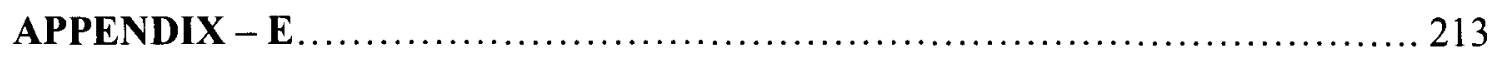

Nomenclature. 213

CURRICULUM VITAE 214 


\section{LIST OF TABLES}

1. Table 2.1. Some popular CFD codes and their developers................... 16

2. Table 2.2. Popular commercial finite element software providers.............. 28

3. Table 3.1. Initial dimensionless parameter values to be examines for determining fluid transitions........................................................ 58

4. Table 3.2A. Initial cases for Setl and related system properties.............. 59

5. Table 3.2B. Transition cases for Set 1 and system properties................. 59

6. Table 3.3A. Initial cases for Set2 and related system properties............... 64

7. Table 3.3B. Transition cases for Set2 and system properties................. 64

8. Table 3.4A. Initial cases for Set3 and related system properties............... 68

9. Table 3.4B. Transition cases for Set3 and system properties................. 69

10. Table 3.5A. Initial cases for Set 4 and related system properties.............. 72

11. Table 3.5B. Transition cases for Set 4 and system properties................. 72

12. Table 3.6A. Initial cases for Set5 and related system properties................ 76

13. Table 3.6B. Transition cases for Set5 and system properties.................. 76

14. Table 3.7A. Initial cases for SET6 and related system properties.............. 80

15. Table 3.7B. Transition cases for SET6 and system properties................ 80

16. Table 4.1. RMS values of wall shear stress at different orbital speeds and radial

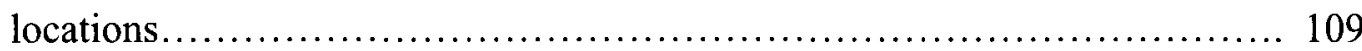

17. Table 4.2. DOSI at different orbital speeds and radial locations............... 114 
18. Table 4.3A. Deviances of Poisson log-linear model of cell proliferation, gamma distribution of cell area and Gaussian distribution for cell shape index responses to DOSI, shear magnitudes, and orbital speeds.......................... 120

19. Table 4.3B. Individual and interacting effects of DOSI, shear magnitude, and orbital speed on cell proliferation, cell area and cell shape index

20. Table 4.4A. Log likelihood of circular regression models of cell orientation to DOSI, shear magnitude on orbital speed.

21. Table 4.4B. Individual and interacting effects of DOSI, shear magnitude and orbital speed on cell orientation by circular regression model.

22. Table 5.1. DOSI at $100 \mathrm{rpm}$ orbital speeds and at radial locations of $20 \%, 60 \%$, and $85 \%$. 146

23. Table 5.2. ANOVA analysis of cell proliferation and cell area responses in the presence of $\mathrm{LTB}_{4}$ and LPS to DOSI and shear magnitude.

24. Table 5.3. ANOVA analyses of gene expression responses to DOSI, shear magnitude, treatment, and replicate. 


\section{LIST OF FIGURES}

1. Figure 2.1. The pipeline of CFD solution............................... 17

2. Figure 2.2. Continuous domain ........................................ 23

3. Figure 2.3. Discrete domain......................................... 24

4. Figure 2.4. Illustration of finite volume method.......................... 26

5. Figure 2.5. Model of orbital motion of a Petri-dish......................... 38

6. Figure 2.6. Example of liquid height profiles throughout one orbit............. 38

7. Figure 2.7. Resultant WSS magnitude and free surface contours at $120 \mathrm{rpm} \ldots . .41$

8. Figure 2.8. Resultant WSS magnitude for different radii at $210 \mathrm{rpm}$ throughout one orbit.

9. Figure 2.9. Resultant WSS magnitude as a function of radius and orbital velocity at radial locations of $16.4 \mathrm{~mm}$ and $4.25 \mathrm{~mm}$ throughout one orbit

10. Figure 2.10. Comparison of area average WSS magnitude from the computational model with values from analytical solution.............................. 46

11. Figure 2.11. Comparison of computational and experimental tangential WSS magnitudes at $12 \mathrm{~mm}$ from the center of the dish at $210 \mathrm{rpm}$

12. Figure 2.12. Comparison of computational and experimental WSS magnitudes at

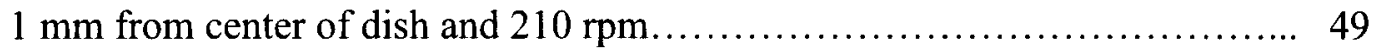

13. Figure 3.1. Dish with seven parameters............................... 53

14. Figure 3.2. Cube of dimensionless parameters............................ 54 
15. Figure 3.3. Example of Froude effect on fluid flow

16. Figure 3.4. Example of Slope ratio effect on fluid flow.................. 56

17. Figure 3.5. Example of Stokes effects on fluid flow $\ldots \ldots \ldots \ldots \ldots \ldots \ldots \ldots \ldots$

18. Figure 3.6. Resultant WSS contours below, at and above the Slope ratio transition for constant low Froude Number of 0.1 and low Stokes Number of

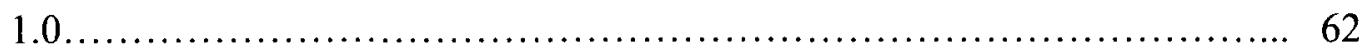

19. Figure 3.7. Tangential vs. radial WSS plots below, at, and above the transition at four radial locations for Set1....

20. Figure 3.8. Resultant WSS contours below, at and above the Slope ratio transition for constant low Froude Number of 0.1 and high Stokes Number of 10.0 66

21. Figure 3.9. Tangential vs. radial WSS plots below, at, and above the transition at four radial locations for Set2. 67

22. Figure 3.10. Resultant WSS contours below, at and above the Froude transition for constant low Slope ratio of 0.1 and low Stokes Number of $1.0 \ldots \ldots \ldots \ldots \ldots 70$

23. Figure 3.11. Tangential vs. radial WSS plots below, at, and above the transition at four radial locations for Set3

24. Figure 3.12. Resultant WSS contours below, at and above the Froude transition for constant low Slope ratio of 0.1 and high Stokes Number of 10.0

25. Figure 3.13. Tangential vs. radial WSS plots below, at, and above the transition at four radial locations for Set4

26. Figure 3.14. Resultant WSS contours below, at and above the Stokes transition for constant low Slope ratio of 0.1 and low Froude Number of 0.1 78 
27. Figure 3.15. Tangential vs. radial WSS plots below, at, and above the transition at four radial locations for Set5. 79

28. Figure 3.16. Resultant WSS contours below, at and above the Stokes transition for constant low Slope ratio of 0.1 and high Froude Number of $1.2 \ldots \ldots \ldots \ldots . \ldots 2$

29. Figure 3.17. Tangential vs. radial WSS plots below, at, and above the transition at four radial locations for Set6.

30. Figure 4.1. Frequency density distribution of cell shape index, normalized cell area, and angle of cell orientation

31. Figure 4.2. Resultant wall shear stress contours $\left(\mathrm{dyn} / \mathrm{cm}^{2}\right)$ viewed from below the dish and corresponding resultant shear waveforms at different radial locations and orbital speeds.

32. Figure 4.3. Bidirectional shear waveforms at different radial locations for different orbital speeds

33. Figure 4.4. An example plot of tangential Vs. radial wall shear stress at different radial locations at $50 \mathrm{rpm}$ orbital speed.

34. Figure 4.5. An example plot of WSS towards $1^{\text {st }}$ principal axes Vs. WSS towards $2^{\text {nd }}$ principal axes at different radial locations at $50 \mathrm{rpm}$.

35. Figure 4.6. Directional oscillatory shear index (DOSI) at different radial locations and orbital speeds.

36. Figure 4.7. Effects of DOSI and shear magnitude on cell proliferation, area, and shape index at three orbital speed at three radial locations.

37. Figure 4.8. Angle of cell orientation relative to tangential flow direction at different orbital speeds and at different radial locations 
38. Figure 5.1. Formation of atherosclerosis plaque in a human artery

39. Figure 5.2. Pathways for synthesis of $\mathrm{LTB}_{4}$

40. Figure 5.3. Flow patterns of different fluid flows through different arteries and aortic arch.

41. Figure 5.4. Resultant wall shear stress contours, plots of radial Vs. tangential WSS components, and Plots of WSS towards $1^{\text {st }}$ principal axes and $2^{\text {nd }}$ principal axes. 146

42. Figure 5.5. Cell images at two radial locations and DOSI levels at $100 \mathrm{rpm}$ orbital speed. 149

43. Figure 5.6. Cell proliferation and area in the presence of $\mathrm{LTB}_{4}$ and LPS under different DOSI levels and shear magnitudes at two radial locations.

44. Figure 5.7. Effects of DOSI and shear magnitudes on gene expressions at mRNA level at different radial locations at orbital speed of $100 \mathrm{rpm}$ 154 


\section{CHAPTER ONE}

\section{INTRODUCTION}

The effects of hemodynamic forces on cellular responses have been studied for the last three decades. Wall shear stresses (WSS) are commonly accepted as the primary influence affecting anchored cells subjected to fluid flow. The significant impacts of WSS on cellular responses in altering the cell proliferation, morphology, gene expressions have been well documented. In addition, previous investigations indicated that cellular responses were altered in different ways under the exposure of different kinds of fluidic shear that includes steady unidirectional shear, oscillatory unidirectional shear, and pulsatile unidirectional shear.

While studying the effects of WSS on cellular responses, shear needed to be generated experimentally. A number of experimental setups have been used to generate different types of shear flow which mainly includes parallel plate flow chambers, cone and plate flow chambers, and orbital shakers. The parallel plate flow chamber generates unidirectional shear simply and controllably, but care must be taken to account for entrance length and the variation of shear across the transverse direction in the channel. The cone and plate apparatus gives nearly uniform tangential shear fields when operated at moderate to low speeds. Oscillatory or pulsatile flow can be created in either apparatus with special pumps or drive motors. A disadvantage of these two setups is that only one experiment can be conducted in the flow chamber at a time, which leads to long time 
requirements for large arrays of experiments. Orbital shakers, on the other hand, are prevalent throughout the cell culture industry and can be used to investigate several cases simultaneously since many culture dishes can fit on its platform. Moreover, the generated shear on the bottom of the dish is pulsatile and bidirectional, which mirrors the character of flow in some parts of the human vasculature. The usefulness of the orbital shaker to generate shear on cells is well documented. Despite the advantages of orbital shakers, it is extremely difficult to quantify the oscillatory shear analytically. Though some analytical approaches exist to quantify steady unidirectional shear, no analytical technique is currently available to quantify oscillatory shear.

Computational Fluid Dynamics (CFD) was applied in this effort to overcome those limitations. With CFD software, a model was created that provides complete temporal and spatial resolution of exerted shear on the bottom of the orbiting dishes where anchored cells reside. This computational model also allowed the system to be examined as unsteady state allowing assessment of oscillating WSS values. The model was further compared and validated with experimentally and analytically determined shear.

Since the generated shear is bidirectional and pulsatile, an effort was made in the next part of the study to understand the effects of directionality of shear and magnitude of shear, on cellular responses including cell proliferation and morphology. The current study was further extended to understand the effects of oscillating shear on inflammatory disease like atherosclerosis in the presence of atherogenic regulators like leukotriene $\mathrm{B}_{4}$ $\left(\mathrm{LTB}_{4}\right)$ and lipopolysaccharide (LPS) as it has been well documented that shear has important effects on atherosclerotic plaque formation. Cell proliferation under different 
oscillating shear ranges at different radial locations of the dish in the presence of $\mathrm{LTB}_{4}$ and LPS was investigated. Furthermore, the shear effects on different atherogenic genes (E-Selectin, I-CAM1, and IL-6) at the mRNA level were investigated in the presence of $\mathrm{LTB}_{4}$ and LPS at different radial locations of the dish.

In the next part of the study, the fluid motion inside the dish was modeled by investigating the system properties that affect the WSS. Fluid flow inside an orbiting dish depends on mainly seven system properties including radius of orbit, orbital speed, dish diameter, viscosity, fluid height, density, and gravity. It is therefore desirable to identify the effects of each of those parameters on WSS. Considering each combination of those parameters would require a massive computational effort, so it was desired to reduce the number of variables to decrease the computational effort. This was accomplished by dimensional analysis which can reduce the seven system properties to four dimensionless parameters: Slope ratio, Froude Number, Stokes Number and Reynolds Number. By considering only laminar flow cases, the Reynolds Number can be kept as constant; leaving just three independent dimensionless parameters. By examining free surface plots for a range of low to high values for each of the parameters, transition points of each of the parameters could be determined. The transition points allow characterizing WSS in terms of high and low ranges for each combination of dimensionless parameters.

Plots of radial versus tangential WSS components were analyzed to assist in understanding to understand flow patterns for the various dimensionless parameter combinations; they also helped to verify the transition point for each of the cases. 


\section{OBJECTIVES:}

The specific objectives of this study are therefore provided below:

1. Generation of spatial and temporal resolution of oscillatory fluidic shear in orbiting dishes by CFD and comparison of computationally derived shear with experimentally and analytically determined shear.

2. Development of a novel hemodynamic parameter - Directional Oscillatory Shear Index (DOSI) to study the effects of directionality of shear on biological responses - cell proliferation and morphology.

3. Study the effects of DOSI and shear magnitudes of oscillating shear on cell proliferation, morphology, and atherogenic gene expressions at the mRNA level in the presence of $\mathrm{LTB}_{4}$ and LPS.

4. Analyze fluid dynamics in orbiting Petri-dishes in order to characterize WSS as a function of Stokes number, Froude Number, and Slope Ratio. 


\title{
CHAPTER TWO
}

\author{
GENERATION OF SPATIAL AND TEMPORAL RESOLUTION OF \\ OSCILLATORY FLUIDIC SHEAR IN ORBITING DISHES BY CFD AND \\ COMPARISON WITH EXPERIMENTALLY AND ANALYTICALLY \\ DETERMINED SHEAR.
}

\subsection{INTRODUCTION AND BACKGROUND}

\subsubsection{Definition of Shear stress}

Shear stress is one of the important properties of any real fluid (liquid or gas) that moves along any solid boundary where the fluid has zero velocity relative to the boundary wall (no slip condition). For instance, in a pipe flow, the fluid velocity is zero at its boundary, whereas it is increased as it moves towards the center. This change of velocity across the direction of the flow is called a velocity profile. The local velocity gradient is known as shear rate, which is reciprocal of the slope of the velocity profile. According to McCabe, et al. (1), for a steady one-dimensional laminar flow of an incompressible fluid along a solid plane surface, shear rate is defined as:

$$
\frac{d u}{d y}=\lim _{\Delta y \rightarrow 0} \frac{\Delta u}{\Delta y}
$$


A shear force therefore exists whenever there is shear in actual flow field. Shear stress is defined as a stress applied parallel or tangentially to a face of a plane and it differs from normal stress which is applied perpendicularly. Shear stress is defined as force per unit area and the formula to calculate the average shear is:

$$
\tau=\frac{F}{A}
$$

where

$\tau=$ shear stress

$F=$ the force applied

$A=$ the area of cross section

Further, for a laminar flow, at steady motion of the fluid, it was shown that (Bird, et al.

$$
\frac{F}{A}=\mu \frac{V}{Y}
$$

where $\mu=$ viscosity of the fluid.

Replacing by new notation, the above equation will be as below:

$$
\tau_{y x}=-\mu \frac{d v_{x}}{d y}
$$

This is known as Newton's law of viscosity.

\subsubsection{Experimental methods to determine fluidic shear stress}

A number of experimental setups have been used to generate different types of shear flow which mainly includes parallel plate flow chambers, cone and plate apparatus, and orbital shakers. The parallel plate flow chamber generates unidirectional shear simply 
and controllably, but care must be taken to account for entrance length and the variation of shear across the transverse direction in the channel. Ohashi, et al. (3) reviewed the experimental and numerical approaches to remodel vascular endothelial cells exposed to fluid shear stress. The discussion included the effects of fluid stress exerted on the endothelial cells which were generated by two experimental configurations - parallel plate and con- and plate flow chambers. Levesque, et al. (4) used a parallel plate flow chamber to exert laminar, steady unidirectional shear stress on endothelial cells to understand the effects of shear on cell morphology. The chamber consisted of a channel with a rectangular cross section and the wall shear stress was determined by the following equation:

$$
\tau_{w}=\frac{6 \mu^{2}}{\rho h^{2}} \operatorname{Re}
$$

where

$\tau_{\mathrm{w}}=$ Wall shear stress

$\mu=$ Fluid viscosity

$\rho=$ Fluid density

$h=$ Height of the rectangular cross sectional channel of the flow chamber

$\operatorname{Re}=$ Reynolds number

Parallel plate flow chambers were widely used thereafter as shear generating equipment for studying the effects of unidirectional shear on different biomedical responses (5-20).

Another type of popular shear generating equipment is the cone and plate apparatus (21-23). It consists of a rotating cone and a stationary plate. The local relative velocity increases linearly with the distance from the cone axis. Thereby it can provide 
constant shear stress. Moreover it gives nearly uniform tangential shear fields when operated at moderate to low speeds. Ley, et al. (24) used a cone and plate apparatus to generate shear stress on endothelial cells to understand shear - dependent inhibition of granulocyte adhesion to cultured endothelium by dextran sulfate. This study indicated that a uniform shear field over the entire cross-sectional area can be generated by a cone and plate flow chamber. The average shear stress was calculated from the actual geometry of the plate and cone apparatus using the area weighted (A) mean shear stress $\tau:$

$$
\tau^{\prime}=\frac{\Sigma(\tau(r) \cdot A(r))}{\Sigma A(r)}
$$

where

$$
\tau(r)=\gamma(r) \eta
$$

with shear rate

$$
\gamma(r)=\frac{2 \cdot \pi \cdot r \cdot f}{h}
$$

where

$\eta=$ Buffer viscosity $=7.5^{*} 10^{-3}$ dynes.s.cm $\mathrm{cm}^{-2}$ at $37^{\circ} \mathrm{C}$.

$\mathrm{h}=\mathrm{Slit}$ height $=50 \mu \mathrm{m}$ for the flat center $(\mathrm{h}=\mathrm{r} . \alpha$ for conical part $)$

$\alpha=$ Cone angle in radian.

$f=$ Rotational frequency.

Oscillatory or pulsatile flow can be created in either of the above apparatus with special pumps or drive motors. 
Another kind of apparatus was used by Owatverot, et al. (25) to generate laminar steady or oscillatory fluidic shear stress in their study to understand the effects of combined cyclic stretch and fluid shear stress on endothelial cell morphology. They used a custom-built device to stretch cells which were cultured on a silicone membrane. The device had the ability to apply in-plane, uniaxial deformation to the central region of the membranes. Since the membrane was incompressible, uniaxial deformation was maintained in the central region of the membrane. The device was intended to produce a Couette flow condition in which the shear stress was predicted by the equation:

$$
\tau=\frac{\mu V}{h}
$$

where

$\mu=$ Fluid viscosity

$\mathrm{V}=$ Belt velocity

$\mathrm{h}=$ Gap height

A novel multi-directional flow chamber (MDFC) was developed by Langerquist, et al. (26) to investigate the endothelial cell morphology dependence on differential shear forces. The design of this kind of flow chamber was based on two flow conditions Couette flow for the rotational component and Poiseuille flow for the axial component. Multi- directional flow was thereby tested with two ratios of axial/rotational shear stress magnitudes. Hsiai, et al. (27) utilized bulk and surface micromaching techniques to fabricate the MEMS shear stress sensor which were operated based on the heat transfer principle. The convective heat from a heated element to the flowing fluid was measured 
and a value of shear stress was measured. They used following linear relation used by Haritonnidis, et al. (28):

$$
\tau^{1 / 3} \infty \frac{V^{2}}{R}
$$

where

$\tau=$ Wall shear stress

$\mathrm{V}=$ Fluid velocity

$\mathrm{R}=$ radius of the cone

In another study, Chiu, et al. (29) used a novel coculture flow system to exert laminar steady shear stress and its effects on vascular endothelial cells and smooth muscle cells. The main advantage of this system was that the chamber allowed direct observation of the cells during the flow experiment via the observation window. The wall shear stress produced by the chamber was calculated by the formula:

$$
\tau=\frac{6 \mu Q}{w h^{2}}
$$

where,

$\mu=$ Viscosity of the perfusate

$\mathrm{Q}=$ Flow rate

$\mathrm{w}=$ Channel width

$\mathrm{h}=$ Channel height 
A novel shear generating method was developed by Inoguchi, et al. (30). They used a custom designed pulsatile circulatory apparatus which was composed of a centrifugal pump, a tube installer chamber, a compliance tank, a flow control valve, and a reservoir. The steady flow at a fixed shear stress was produced by adjusting the rotational speed of the centrifugal pump. The shear stress was determined by the following equation:

$$
\tau=\frac{(4 \eta Q}{\left(\pi r^{3}\right)}
$$

where,

$\eta=$ Viscosity of the medium

$\mathrm{Q}=$ Fluid flow rate

$r=$ Internal radius of the tube.

A disadvantage of all the above arrangements is that only one experiment can be conducted at a time, which may cause long time requirements for large arrays of experiments. Orbital shakers are free from that limitation and are prevalent in the cell culture industry because of their simplicity of use. The major advantage of using an orbital shaker is that several cases can be studied simultaneously since several dishes can fit on a platform and are easily stackable. Moreover, it can be applied to study the effects of oscillatory flow which is similar to the pulsing in the human vasculature. Since generated shear in orbital shakers is not uniform and is oscillatory in nature, it is difficult to accurately determine the magnitude of shear analytically. Ley et al. (24) investigated shear-dependence of endothelial cells in both orbital shaker and cone-and-plate experiments. The shear stresses they reported in the orbital shaker experiments were uniform and estimated analytically $(24,31)$ as 


$$
\tau_{\psi^{*}}=a^{*} \sqrt{\rho^{*} \mu^{*}(2 * \pi * f)^{3}}
$$

where $\tau_{w}$ is shear stress, a is radius of the cylinder, $\rho$ is fluid density, $\mu$ is fluid viscosity and $f$ is frequency of rotation.

Equation (2.11) is known as the solution of Stokes Second problem which describes the orbital motion of a plate of infinite width having a thin layer of fluid. According to Stokes Second problem, Equation (2.11) expresses constant magnitude of shear. Thus, it cannot predict the unsteady oscillatory nature of shear nor can it predict shear at various locations of the dish. Furthermore, no wall effects have been assumed there. Also the equation is limited to the specific condition of low Slope Ratio, low Froude Number, high Stokes Number and low Reynolds Number.

\subsubsection{Advantages of using orbital shakers for generating shear}

Shear on the bottom of the dish is pulsatile and bidirectional, which mirrors the character of flow in some parts of the human vasculature. The usefulness of the orbital shaker to generate shear on cells has been well documented. To investigate the effects of shear stress on 1-Matrix Metalloproteinase expression in the endothelium, Yun, et al. (32) used an orbital shaker to generate shear stress to understand the effect of shear induced transcription factor Spl phosphorylation on membrane type 1-matrix metalloproteinase (MT1-MMP). The results illustrated the complex control MTI-MMP expression in endothelium in response to distinct environmental stimuli (cyclic strain versus shear stress), consisting of both the modulation of specific transcription factor expression (Egr-1) as well as transcription factor post-translational modification (serine phosphorylation of Sp1). In another study, Pearce, et al. (33) used an orbital shaker to 
generate shear stress to study the effects of shear on Cytosolic Phospholipase $\mathrm{A}_{2}\left(\mathrm{cPLA} \mathrm{A}_{2}\right)$ and MAP kinase in human endothelial cells. Kraiss, et al. (34) employed an orbital shaker apparatus to study the effects of shear on a regulator of endothelial cell translation, p70/p85 S6 kinase. Further, Haga, et al. (35) applied an orbital shaker to study the effects of shear on smooth muscle cell proliferation and Akt Phosphorylation. In addition, Dardik, et al. (36) compared the effects of shear stress on endothelial cell responses between an orbital shaker apparatus and in a parallel plate channel. They found that orbital shear increased cell proliferation by $29 \%$ compared to ECs in the parallel plate channel. Further, they observed that cells in the parallel plate channel were aligned towards the direction of shear whereas cells in the center of the orbiting dish were unaligned, similar to cells not exposed to shear stress.

\subsubsection{Computational Fluid Dynamics (CFD)}

Computational fluid dynamics (CFD) is a branch of fluid mechanics which uses numerical methods and algorithms in computational technology to solve and analyze problems that involve fluid flow. Using CFD software, a virtual computational model of systems of interest can be created. Further, by applying the physics and chemistry of relevant fluid flow through this virtual prototype, it is possible to predict fluid dynamics and related physical phenomena. CFD software has the ability to simulate the flows of gases and liquids, heat and mass transfer, moving bodies, multiphase physics, chemical reactions, fluid-structure interaction, and acoustics through computational modeling in most proper and accurate way. Over the last forty years, CFD has been used in both industrial and academic applications. The development of CFD began with the advent of 
digital computers in the early 1950s. The physical aspects of any fluid flow are governed by conservation of mass, Newton's second law, and conservation of energy. These fundamental governing equations of fluid flow are expressed in terms of partial differential equations which are solved numerically in CFD, the values of which are advanced in space and/or time to obtain a final numerical description of the complete flow field of interest (37). The basic tools for solving the partial differential equations in CFD are the finite difference method (FDM) and the finite element method (FEM). Both methods have been developed extensively for solving problems in fluid dynamics, heat transfer, and other applications (38). The high speed digital computer is the key instrument to perform CFD applications since the CFD solutions need the repetitive manipulation of millions of numbers during simulation. Therefore, the advances of CFD and its applications are essentially related to the advances of computer hardware particularly in regard to storage and execution speeds (39). The major application of CFD was first done by Kopal, et al (40). He compiled very large tables of supersonic flow over a sharp cone by solving the governing differential (41) equations numerically which were carried out by a primitive digital computer at the Massachusetts Institute of Technology. The first effective and accurate CFD solutions however were found in the 1950 s and early 1960 s by the simultaneous advent of high speed and efficient computers. Researchers applied CFD then to solve the solutions of high velocity and high temperature body problems that included boundary problems and inviscid flow problems (42-45). The second generation of CFD solutions involved the complex applications of different fluid dynamic problems including subsonic flow, supersonic flow, and viscous flow. Handling of those problems were not amenable to just boundary layer 
approximation. Therefore, the full Navier - Stokes equations and time dependent techniques were incorporated in the mid 1960s which were revolutions in flow field calculations (46). In spite of applying CFD to classical cases of pure experimental or pure theoretical problems, CFD rapidly became a popular and effective tool used to analyze various engineering problems; some such applications of CFD are given by Fluent (4749):

- Aerospace and turbo machinery

- Process design and process equipment design

- Biomedical Science and Engineering

- Automobile - power train and aerodynamics/thermal

- Power generation and oil and gas industry

- Heat transfer and heat exchanger design

- Health care and water

- Electronics and semiconductor

- HVAC and appliances

- Materials processing including glass, plastic, and materials

- Space, defense, and marine

- Architectural design and fire research

Furthermore, CFD can be applied to designing and modeling external/internal automotive flows and in-cylinder flows, high speed aerodynamics, rocket flows, multiphase flows, reacting flows, chemical reactors, bubble columns, mixing vessels, fluidized beds, packed beds, stirred tank, catalytic reactors and many more. CFD has 
especially important applications in chemical process industries in the design of various processes and process equipment.

The Navier-Stokes equations are the governing equations of any CFD solution in solving single and multiphase fluid flow. Those partial differential equations are then discretized by a finite difference method or a finite element method as described previously. The discritization of the spatial domain into small cells forms a computational mesh or grid, and a suitable algorithm is then applied to solve the equations of motion numerically. With rapid demand of CFD solutions in different applications, many effective commercial CFD codes are available nowadays. Table 2.1 provides some of these popular codes generated by different CFD development organizations:

Table 2.1. Some popular CFD codes and their developers.

\begin{tabular}{ccc}
\hline Developer & Code & Web Address \\
\hline ANSYS & FLUENT & www.ansys.com \\
ANSYS & CFX & www.ansys.com \\
CD-Adapco & STAR-CD & www.cd-adapco.com \\
CFDRC & CFD-ACE & www.cfdrc.com \\
CHAM & PHOENICS & www.cham.co.uk \\
Flow Science & FLOW 3D & www.flow3d.com \\
ADINA R\&D Inc & ADINA CFD & www.adina.com \\
EXA & Power Flow & www.exa.com \\
\hline
\end{tabular}




\subsubsection{Methodology of obtaining a CFD solution for a particular system of interest}

$(47,49)$

The fundamental method of any CFD solution is expressed in Figure 2.1. It mainly consists of three major steps: preprocessing, CFD simulation, and post processing. Stage 1 to stage 4 reflects the preprocessing, stage 5 indicates CFD simulation, and stage 6 shows the post processing step.

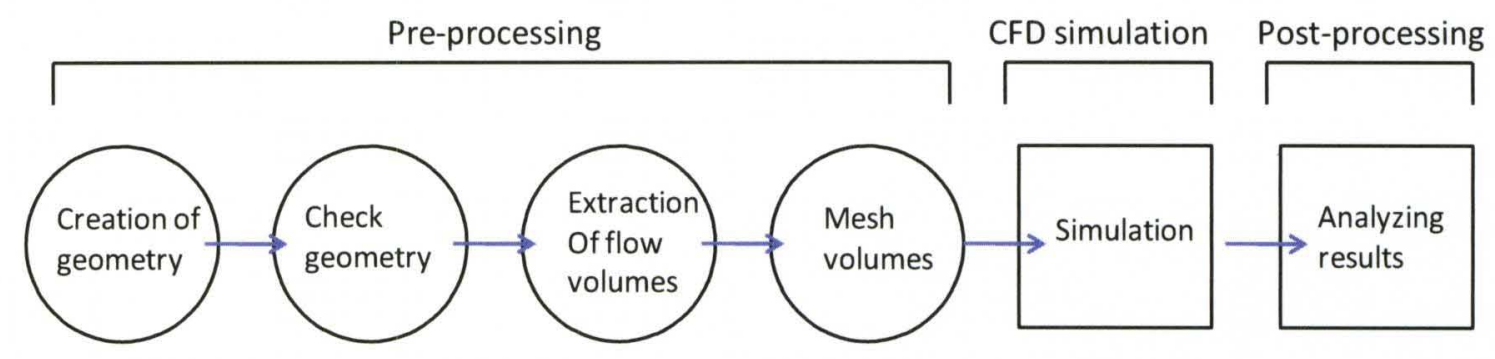

Figure 2.1. The pipeline of CFD solution

- Preprocessing:

i) Creation of the geometric physical domain and the flow regions:

Definition and creation of geometry of flow region is the first step of any CFD analysis. Creation of geometry for any CFD calculation allows fully developed flow over its computational domain.

ii) Mesh Generation:

Mesh generation is one of the most important steps during preprocessing after creation of the domain geometry. In order to solve the flow physics, it is 
required to subdivide the domain into smaller, non overlapping discrete cells which are called control volumes. The mesh or grid is the summation of those control volumes. The equations representing fluid flow through each of the cells are then numerically solved to achieve the discrete values of different flow properties such as velocity, temperature, pressure, and other transport parameters of interest. The accuracy of any CFD solution, therefore, strongly depends on the structure of the mesh. Most commercial CFD codes have their own CAD interface to import the mesh from a solid modeler package. Some of the well known modeling packages are GAMBIT, SOLIDWORKS, PARASOLID, SOLIDEDGE, PRO-ENGINEER and UNIGRAPHICS.

The mesh types include 2D triangular/quadrilateral, 3D tetrahedral /triangular/ hexahedral/wedge/polyhedral, and mixed mesh. The mesh can be structured, unstructured, or a hybrid mesh. For relatively simple geometry, a structured mess is used which is characterized by regular connectivity and is expressed as a two or three dimensional array of a regular distribution of rectangular cells. The major limitation of this kind of mesh is the inability to generate a 2D quadrilateral mesh or a 3D hexahedral mesh. An unstructured mesh is used to handle complicated geometry. It is characterized by -irregular connectivity's which cannot be expressed in simple two or three dimensional arrays. The advantage of unstructured meshes is that they are automated and unlike structured ones, no code development is necessary, therefore it requires little time. Further, it is able to provide solutions of very large and complex problems in a relatively short span of time. On the other hand, the major 
drawback of unstructured meshes is the lack of user control when laying out the mesh. In a hybrid mesh structure; there are existences of both structured and unstructured portions of the mesh. It is designed to take advantage of the positive aspects of both structured and unstructured meshes. The disadvantages of a hybrid mesh are that it is difficult to use and requires advanced level expertise in handling various structured and unstructured grid locations and properties.

iii) Physical Modeling :

The third step of preprocessing is defining the physical model. The governing equations such as equations of motion, conservation of mass, and conservation of energy are incorporated in the model in this step.

iv) Specification of boundary conditions :

Appropriate boundary conditions need to be incorporated to model real physical interpretations of the fluid flow of interest and to simulate the flow properly. Inflow and outflow boundary conditions within the flow domain are incorporated to understand the entering and outgoing fluid behavior. Appropriate boundary conditions are also essential to assign the external stationary solid walls which bound the flow geometry. 


\section{- Numerical Simulation:}

After preprocessing, the properly prepared mesh will be exported to any commercial CFD solver through its CAD graphical interface, and after assigning the appropriate properties, proper residual values and proper time step, the simulation can be started by which the governing equations are solved iteratively either in steady or in unsteady conditions.

\section{- Post-processing:}

After completion of flow simulation, a post processor is used to analyze and visualize the solution.

\subsubsection{Basic equations that control fluid flow in CFD}

Navier-Stokes equations govern the fluid flow of interest in CFD. The basic controlling equations include conservation of mass, momentum, and energy (48). The equations are as follows.

For conservation of mass:

$$
\frac{\partial \rho}{\partial t}+\frac{\partial}{\partial x_{i}}\left(\rho u_{i}\right)=0
$$

For Conservation of momentum:

$$
\rho \frac{\partial u_{i}}{\partial t}+\rho u_{j} \frac{\partial u_{i}}{\partial x_{j}}=-\frac{\partial p}{\partial x_{i}}+\frac{\partial \tau_{j i}}{\partial x_{j}}+\rho g_{i}
$$

where the shear stress $\tau_{\mathrm{ij}}$ is: 


$$
\tau_{j i}=\left[\mu\left(\frac{\partial u_{i}}{\partial x_{j}}+\frac{\partial u_{j}}{\partial x_{i}}\right)\right]-\frac{2}{3} \mu \frac{\partial u_{j}}{\partial x_{j}} \delta_{i j}
$$

For Conservation of energy:

$$
\frac{\partial}{\partial t}(\rho h)+\frac{\partial}{\partial x_{i}}\left(\rho u_{i} h\right)=\frac{\partial p}{\partial x_{i}}\left(k \frac{\partial T}{\partial x_{i}}\right)-\frac{\partial}{\partial x_{i}} \sum h_{j} J_{j}+\frac{\partial p}{\partial t}+u_{i} \frac{\partial p}{\partial x_{i}}+\tau_{i j} \frac{\partial u_{i}}{\partial x_{i}}+S_{h}
$$

where,

$$
h=\sum m_{i} h_{i} \text { and } h_{i}=\int_{T_{r e t}}^{T} C_{p i} d T
$$

Conservation of chemical species:

$$
\frac{\partial}{\partial t}\left(\rho m_{i}\right)+\frac{\partial}{\partial x_{i}}\left(\rho u_{i} m_{i}\right)=\frac{\partial}{\partial x_{i}}\left(J_{i^{\prime}, i^{\prime}}\right)+S_{i^{\prime}}
$$

where

$$
J_{i^{\prime} i^{\prime}}=-\rho D_{i^{\prime}} m \frac{\partial m_{i}}{\partial x_{i}}
$$

The above partial differential equations are reduced to linear algebraic equations by a finite difference method. The algebraic equations, therefore, are in the form:

$$
\phi_{p} \sum_{i}\left(A_{i}-S_{p}\right)=\sum_{i} A_{i} \phi_{i}+S_{c}
$$

where $A_{i}$ is the coefficient contributed from diffusive and convective fluxes.

$S_{p}$ and $S_{c}$ are related as below:

$$
S_{\phi}=S_{c}+S_{p} \phi_{p}
$$


The algebraic summations are performed numerically over each of the neighboring finite difference cells.

A differentiating scheme is used to calculate the derivatives of the flow variables and interpolate between grid points which utilizes the power law. The algebraic equations are numerically solved by a semi-implicit iterative scheme which starts at a defined set of initial conditions and converges to correct solutions after executing a specified number of solutions and tolerance. Each iteration involves specific steps, which are given below:

- Each of $\mathrm{u}, \mathrm{v}, \mathrm{w}$, and momentum equations are solved using initial given pressure values and, thereby, the velocity field is updated.

- Changes in the velocity field essentially cause the changes in pressure and the correction of pressure can be done by using a Poisson type equation derived from continuity and momentum equations. The correction in pressure and velocity, thereby, satisfy the continuity equation.

- Next, auxiliary equations such as equations of enthalpy and chemical species are solved by using previous updated velocities.

- From the new set of values, the fluid properties are updated.

- Finally, the solver checks the set of equations to reach the proper convergence.

- The steps are iterated until the residual difference between the iterations reaches the specified predefined residual value. 


\subsubsection{Methods of discritization of governing partial differential equations}

The fundamental process behind any CFD solution is the discritization of the continuous problem domain. As an example, consider the velocity $(v)$ in any continuous flow domain which can be expressed as:

$$
v=v(x), 0<x<1
$$

The continuous domain is thereby expressed in Figure 2.2.

\section{Continuous domain}

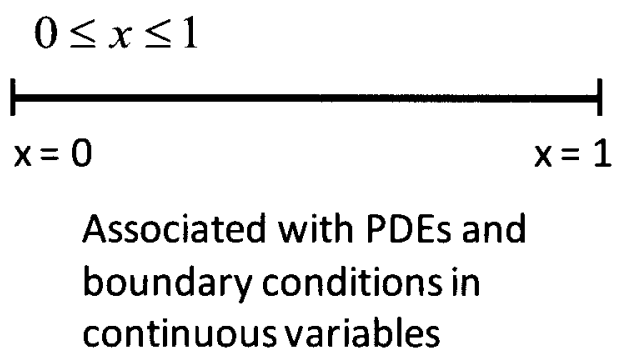

Figure 2.2. Continuous domain

Considering the discrete domain where the flow variable is defined only at the grid points, the velocity will therefore be defined at an assigned number of grid points (say N) and can be expressed as

$$
v_{i}=v\left(x_{i}\right), \text { where } I=1,2, \ldots ., \mathrm{N}
$$

The discrete domain can therefore be expressed as in Figure 2.3. 


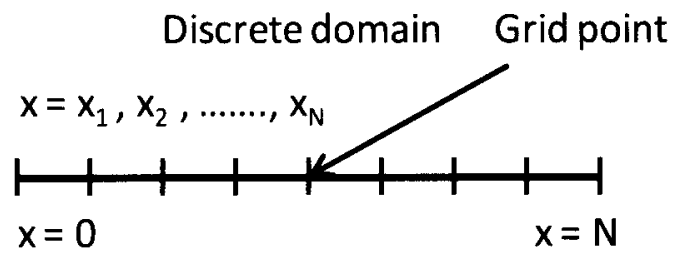

PDEs are converted in linear algebraic equation in discrete variables

Figure 2.3. Discrete domain

Therefore, a finite difference method is necessary to discretized the NavierStokes equations into linear algebraic equations, which are briefly described below:

\subsubsection{Finite Difference Method (FDM) for the discretization process}

The fundamental approach in discretizing the governing equations in CFD is illustrated by considering a simple one dimensional equation (50):

$$
\frac{d u}{d x}+u^{m}=0 ; 0 \leq x \leq 1 ; \mathrm{u}(0)=1
$$

where the equation is linear with $\mathrm{m}=1$ and nonlinear with equation $\mathrm{m}=2$.

At $m=1$, the discrete representation of the above equation will be in the following grid containing four equally spaced points with $\Delta \mathrm{x}=0.33$.

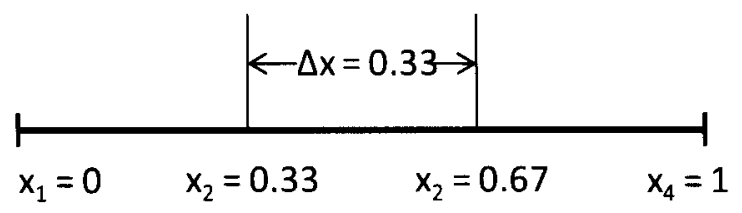

At any grid point, the above governing equation will be

$$
\left(\frac{d u}{d x}\right)_{i}+u_{i}=0
$$


where the subscript $i$ represents the value at any grid point $x_{i}$.

To obtain the expression of $(d u / d x)$ in terms of $u$ at any given grid point, a Taylor's series expansion is employed here, and the expansion is:

$$
u_{i-1}=u_{i}-\Delta x\left(\frac{d u}{d x}\right)_{i}+O\left(\Delta x^{2}\right)
$$

Rearranging,

$$
\left(\frac{d u}{d x}\right)_{i}=\frac{u_{i}-u_{i-1}}{\Delta x}+O\left(\Delta x^{2}\right)
$$

The error $O\left(\Delta \mathrm{x}^{2}\right)$ in the series is a truncation error .

By eliminating the higher order terms in the above Taylor's series expansion, the following discrete equation can be achieved:

$$
\frac{u_{i}-u_{i-1}}{\Delta x}+u_{i}=0
$$

It is important to note that the differential equation here ultimately is converted into a linear algebraic equation. There are two methods widely used by most commercial CFD codes in performing a finite difference method depending on the types of flow past complex geometries: Finite volume method and Finite element method.

\section{Finite Volume method:}

A finite volume method is used by the commercial CFD code, FLUENT. In this method, the quadrilateral grid is called a "cell" and the grid point is referred to as the "node". The cells are usually triangular in 2D, and tetrahedral, hexahedral, or prisms in 3D. In this method, the integral form of the governing conservation equations are applied to the control volume to get the discrete equations for the particular cells. 
In practice, the integral form of the continuity equation for steady incompressible flow can be written as $(50)$ :

$$
\int_{\xi} \cdot \hat{n} d s=0
$$

where, $S$ is the surface of the control volume over which the integration is performed and $\hat{n}$ is the normal at the surface. The above equation implies that the net volume flow into the control volume is zero.

Consider a rectangular cell shown Figure 2.4:

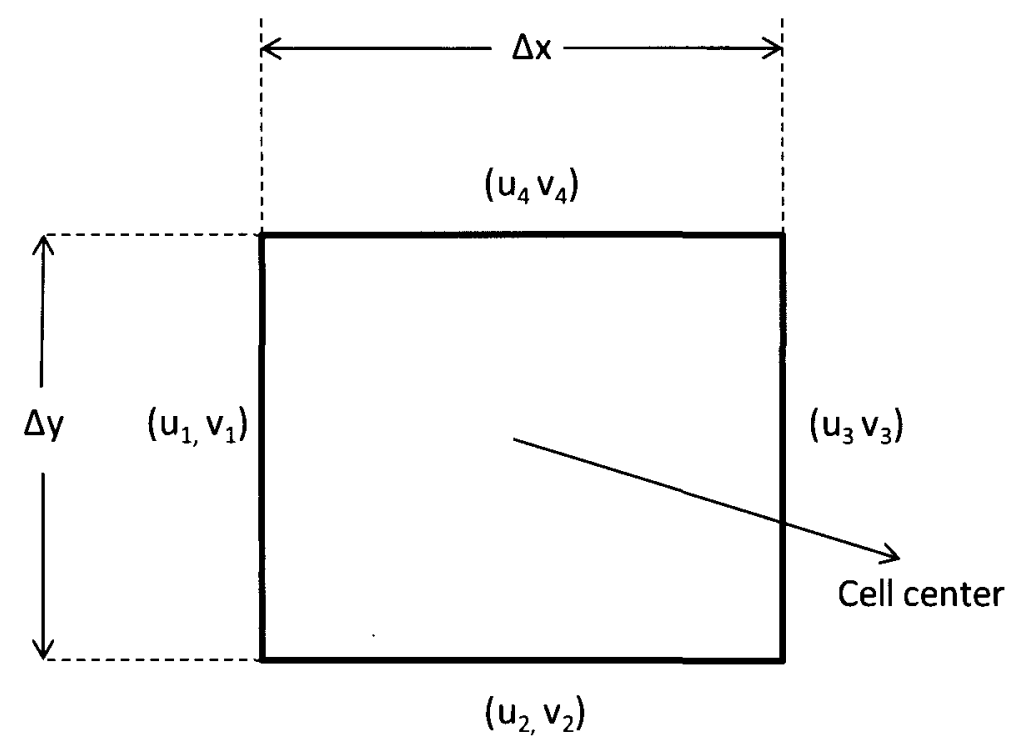

Figure 2.4. Illustration of finite volume method

The velocity at any face $i$ of above rectangle can be expressed as:

$$
\vec{V}_{i}=u_{i} \hat{i}+v_{i} \hat{j}
$$

Applying the mass conservation from equation (2.26), the discrete form of the continuity equation can be achieved in the following form:

$$
-u_{1} \Delta y-v_{2} \Delta x+u_{3} \Delta y+v_{4} \Delta x=0
$$

Equation (2.28) indicates that the net mass flow throughout the control volume is zero, ensuring that the total mass is conserved in the cell. Similarly, other governing 
equations such as conservation of momentum and energy also can be discretized into simple linear algebraic equations by applying the finite volume method.

\section{Finite Element Method (FEM)}

The finite element method is another technique to discretize the governing partial differential equations of a system of interest into simple algebraic linear equations. It was originally developed to study the stresses in complex airframe structures, but nowadays it is widely applied to the broad field of continuum mechanics and different fluid flow due to its specific advantages over the traditional finite difference (finite volume method) method. The finite difference model is used to provide a pointwise approximation to the governing equations and it can be further extended in an array of grid points. Though it is a popular technique in treating the difficult problems to a certain extent, it cannot be used to model irregular geometries having unusual boundary conditions. The finite element method can overcome limitations of the finite difference method. Unlike the finite difference method which creates the solutions over an array of grid points, in finite element method the solution regions are built up on many small, inter-connected subregions or elements.

The most common commercial finite element software providers are listed in Table 2.2 . 
Table 2.2. Popular commercial finite element software providers

\begin{tabular}{ccc}
\hline Company & Product & Website \\
\hline Ansys, Inc & ANSYS & www.ansys.com \\
Adina R\&D, Inc & ADINA FEA & www.adina.com \\
Hibbitt, Karlsson \& Sorensen & ABAQUS & www.hks.com \\
Structural Data Research Corp. & SDRC-Ideas & www.sdrc.com \\
Parametric Technology, Inc. & RASNA & www.ptc.com \\
MSC Software Corp. & MSC/NASTRAN & www.mscsoftware.com \\
\hline
\end{tabular}

The working steps of finite element method are therefore briefly stated below:

- Discretize the continuum region into elements

- Assign nodes to each element and select the interpolation function to represent the field variable over the element

- Determine the matrix equations which express the element properties by employing one of the three approaches such as direct approach, variation approach, or the weighted residual approach.

- Combine all matrix equations expressing the individual element properties and form the matrix equations to obtain the system equations.

- Assign the boundary conditions to the system equations before the numerical solutions.

- Solve the system equations 


\subsubsection{CFD techniques in analyzing multiphase flow}

The modeling of different industrial processes often requires analyzing multiphase flow. That can include liquid-liquid, gas-liquid, and liquid-solid interactions, and proper modeling of multiphase flow is essential for those cases to understand the fluid behavior on different interfaces. In practice, there are two available approaches to model multiphase flows numerically: the Euler-Lagrange approach and the Euler-Euler approach (47).

\subsubsection{The Euler-Lagrange approach}

The Euler-Lagrange approach is used to model the Lagrangian discrete phase. In this model, the trajectory and state of individual particles can be tracked in space and time by integrating the equations of mass, motion, and energy for each particle of the system (51). It has been used to model systems which involve particles. The fluid flow is considered here as a continuous phase by solving time averaged Navier-Stokes equations, whereas the dispersed phase can be solved by tracking different types of particles, bubbles, or droplets. The dispersed phase exchanges the momentum, mass, and energy with the continuous fluid phase (47). The Euler-Lagrange method is applied to model a wide range of industrial equipment and fluid flow $\mathrm{t}$ which essentially involves the discrete phase. Some applications of this model include modeling of spray dryers, coal, and fuel combustion. 


\subsubsection{The Euler-Euler Approach}

Modeling of multiple continuous phases can be done with the Euler-Euler approach. The different continuous phases are considered mathematically as interpenetrating continua. A new concept of volume fraction, which is a continuous function of space and time, is used in this model since the volume of a phase can not be carried out to the other phase. The governing conservation equations of each phase are used to obtain a set of similar equations for all phases. Three different Euler-Euler multiphase models are available depending on the modeling requirements:

\section{- Volume of Fluid model (VOF)}

VOF is a surface tracking technique which is applied to a fixed Eulerian mesh. In this model, a single set of momentum equations is shared by two immiscible fluids and the volume fraction each fluid in each cell is tracked throughout the domain. As a result, the same set of governing equations in a single phase flow is solved for an equivalent flow. The VOF model is applied in modeling stratified flow, free surface flow, sloshing, the liquid motion after breaking of a dam, the prediction of a jet break up, etc. The basic equations related to a VOF model are described below:

$$
\begin{aligned}
& \rho=\sum_{i} \rho_{i} \alpha_{i} \\
& \mu=\sum_{i} \mu_{i} \alpha_{i} \\
& c_{p}=\sum_{i} \frac{\left(c_{p}\right)_{i} \rho_{i}}{\rho} \alpha_{i}
\end{aligned}
$$


where, $\alpha_{i}=\frac{V_{i}}{V}=$ volume fraction, $\rho_{i}, \mu_{\mathrm{i}}$ and $\left(\mathrm{c}_{\mathrm{p}}\right)_{\mathrm{i}}$ are the density, molecular viscosity, and specific heat of the i-th phase.

The transport of volume fraction is described by the following conservation equation:

$$
\frac{d}{d t} \int_{V} \alpha_{i} d V+\int_{S} \alpha_{i}\left(v-v_{g}\right) d a=\int_{V} s_{\alpha_{i}} d V
$$

where $s_{\alpha_{i}}$ is the source or sink of the $\mathrm{i}$-th phase.

- The mixture model

This model is designed for two or more phases and solves the mixture momentum equation to predict the dispersed phase. The mixture model is applied to model bubbly flows, sedimentation, and cyclone separators etc.

\section{- The Eulerian model}

The Eulerian model solves a set of momentum and continuity equations for each phase. It is generally applied to model the granular flows, where the properties are obtained from kinetic theory. Flows through bubble columns, fluidized beds, and particle suspensions are modeled by this method.

\subsubsection{Application of CFD in quantifying wall shear stress}

A lot of complexities arise while quantifying different WSS while studying the effects of fluidic wall shear stress on human vasculature and cellular responses. The complicating structures of different parts and different physiological conditions in the 
human vasculature often make the fluid flow very complex. The flow may be steady and unidirectional, or unsteady and pulsating depending on the location. As a result, the nature of exerted WSS by fluid flow is different in different parts of the human vasculature. It can be steady unidirectional, oscillatory unidirectional, pulsatile unidirectional or oscillatory bi-directional. To understand the effects of those WSS on cellular responses, it is necessary to quantify WSS which is difficult to accomplish by both experimentally and analytically. No previous studies have been reported which measured the pulsatile bi-directional WSS either experimentally or analytically. To counter the deficiency, CFD has been employed for modeling the fluid flow and quantifying WSS. With CFD software, a model can be created that can provide complete temporal and spatial resolution of exerted shear. A computational model also allows the system to be examined as unsteady state allowing assessment of oscillating WSS values. Further, different parts of the human vasculature can be modeled with CFD software and the fluid flow through it can be simulated by mimicking the same vascular physiological conditions. As a result, an accurate quantification of different types of WSS can be achieved by simulated fluid flow.

CFD has been used to determine extensively to determine WSS due to the availability of effective and accurate commercial software. Sakurai, et al. (52) studied the flow pattern over endothelial cells (EC) by using computational fluid dynamics. A preliminary result of the three dimensional computation of the flow field around cultured EC has been reported there. In another effort, Satcher, et al. (53) used CFD to derive the distribution of fluid forces on arterial endothelium to understand the effects of shear stress in endothelial cells. Buschmann, et al. (54) performed an analytical and numerical 
analysis of the steady, laminar, three dimensional flow of a Newtonian fluid at low Reynold's number. Unsteady oscillating and pulsating flow were studied in this effort by numerical simulation. The response of endothelial cells to fluid shear stress was investigated in this study. CFD studies of pulsatile flow had been perfomed by Marshall, et al.(55). The experimental flow pattern and derived WSS were compared with corresponding CFD prediction in this study. In another study, Yamaguchi, et al. (56) applied CFD for the shear stress measurement over cultured endothelial cells. This study suggested the existence of a preferred location of a shear stress sensing apparatus on the cell surface. The morphology of cultured endothelial cells which forms under shear loading, was further investigated by Yamaguchi, et al. (57). Weston, et al. (58) compared steady and pulsatile flow in a pipe with CFD simulations of flow through the pipe. Velocity in the pipe was measured by MRI technique whereas wall shear stresses were determined by CFD. Banerjee, et al. (59) used CFD to evaluate hemodynamic implications of coronary artery balloon angioplasty. The flow field there was in the oscillatory region of the distal vessel. Papathanasopoulou, et al. (60) established a general agreement between MRI technique and CFD simulations when comparing velocity measurements in a physiologically realistic model of human carotid bifurcation with pulsatile flow. Morbiducci, et al. (61) further applied computational fluid dynamics while investigating the existence of a relationship between helical flow structures and vascular wall indexes of atherogenesis in aortocoronary bypass models with different geometric features. The results confirmed that the helical flow constitutes an important flow signature in vessels and its strength as a fluid dynamic index for risk stratification, in the activation of both mechanical and biological pathways leads to fibrointimal hyperplasia. 
In another effort, Brown, et al. (62) studied the flow conditions in a parallel plate bioreactor by using CFD and characterized that experimentally using a computer controlled flow regulation apparatus and associated flow visualization techniques. Berthier, et al. (63) compared the blood flow patterns calculated in a coronary vessel reconstructed by three different methods. The coronary was modeled there by CFD. The results showed that local pressures, wall shear stress and velocity profiles obtained by computational simulation, were severely affected by geographical modification. Jung, et al. (64) used three phase analytical modeling of blood flow. In this effort, a three phase CFD approach which includes pathways used to numerically simulate the local hemodynamics in such a flow regime. Tokuda, et al. (65) further studied three dimensional numerical simulation of blood flow in the aortic arch to understand mechanism of stroke during cardiopulmonary bypass. To do that it was necessary to understand the location of turbulence, wall pressure, and flow distribution within the aortic arch. A finite element method was therefore applied here to simulate the blood flow numerically. The results confirmed that blood flow during cardiopulmonary bypass can be modeled and simulated by applying CFD and a finite element method. Dong, et al. (66) applied CFD to design and manufacture a bioreactor which is widely used in tissue engineering due to their controllability in that environment and operating conditions. The CFD simulations of different circular bioreactors with various parameters were performed in this effort and the concentration status of glucose and lactate in different time and shear stress were obtained here. Nanduri, et al. (67) applied CFD techniques to simulate the biological flow. Since CFD is a potential tool to understand biological flow, robust and appropriate grid generation therefore is needed to characterize the complex 
geometries of different flow fields. A simple MATLAB based grid generation technique suitable for CFD of external and internal biological flows (blood flows, respiration, and flows around the human body) was therefore proposed in this study. In another study, Tricht, et al. (68) compared the hemodynamic in hemodialysis grafts by means of CFD. A validated three-dimensional CFD model was developed to simulate the flow to understand whether differential arterial anastomotic geometries result in different hemodynamics at the arterial (AA) and venous anastomosis (VA) in the two graft types $6 \mathrm{~mm}$ graft (CD) and 4-7 $\mathrm{mm}$ graft (TG). By analyzing the hemodynamic parameters that includes wall shear stress, wall shear stress gradient, and pressure gradient, the simulation showed that TG did not show a better outcome compared to the CD. Dehlagi, et al. (69) further analyzed the wall shear stress in stented coronary artery using 3D CFD model. Blood velocity properties and shear stress values were computed in three different sites in this study. The results showed that the analyses of wall shear stress between stent struts, pre, and post stent regions are essential in stent design. The mechanism of morphological remodeling of endothelial cells that was induced by blood flow was studied by Fukushima, et al. (70). They used CFD to measure the shear stress distributions using the simulated flow field and examined, therefore, how shear stress on the surface of the same group of cells changed. The results showed that there were some cells where shear stress was increased and the morphological changes of each cells was not always adaptive. Smith, et al. (71) used 2D CFD while calculating the wall shear stress in left coronary artery bifurcation for pulsatile flow. The results indicated that the magnitude of wall shear stress has great influence on the development of coronary arterial disease and the selection of a blood viscosity model is a key factor, while simulating the flow by CFD. 
In this part of the study, CFD was employed to simulate the motion of fluid in an orbiting dish. A model was created by FLUENT that provides complete spatial and temporal resolution of WSS over the bottom surface of the dish. The results therefore were compared with experimentally determined shear data and compared to the Stokes second problem analytical solution because of the particular constraints that make it valid for these cases. 


\subsection{MATERIALS AND METHODS}

\subsubsection{Modeling and Computation}

FLUENT 6.2.5 (ANSYS Inc., Ann Arbor, MI, USA) was used to solve the 3D, laminar, incompressible, Newtonian flow. While the flow in the dish is periodic, a fully transient solution was applied. The program solves the governing equations for conservation of mass and momentum which was described previously in equations 2.12 , 2.13 , and 2.14

While the fluid motion is periodic and separable, a convenient and practical approach was adopted in developing the model as an unsteady laminar flow problem in FLUENT 6.2.The clockwise (viewed from below the dish) orbit of the dish was specified in a user-defined function (UDF) in terms of the orbital frequency and orbital radius. The fluid flow in an orbiting petri-dish is unsteady state laminar flow. Simulating flow in the dish required a dynamic grid that moved through space; the geometry and mesh of the dish which was created in the preprocessor GAMBIT. Free surface tracking was accomplished with the volume of fluid (VOF) model in which the two fluids at the interface share boundary conditions for their respective conservation equations. Figure 2.5 indicates the orbital motion in a Petri-dish throughout one orbit and Figure 2.6 shows an example set of profiles of liquid height on the vertical walls of the dish at eight orbital positions. The thin red layer represents the liquid and the white layer indicates the air. 


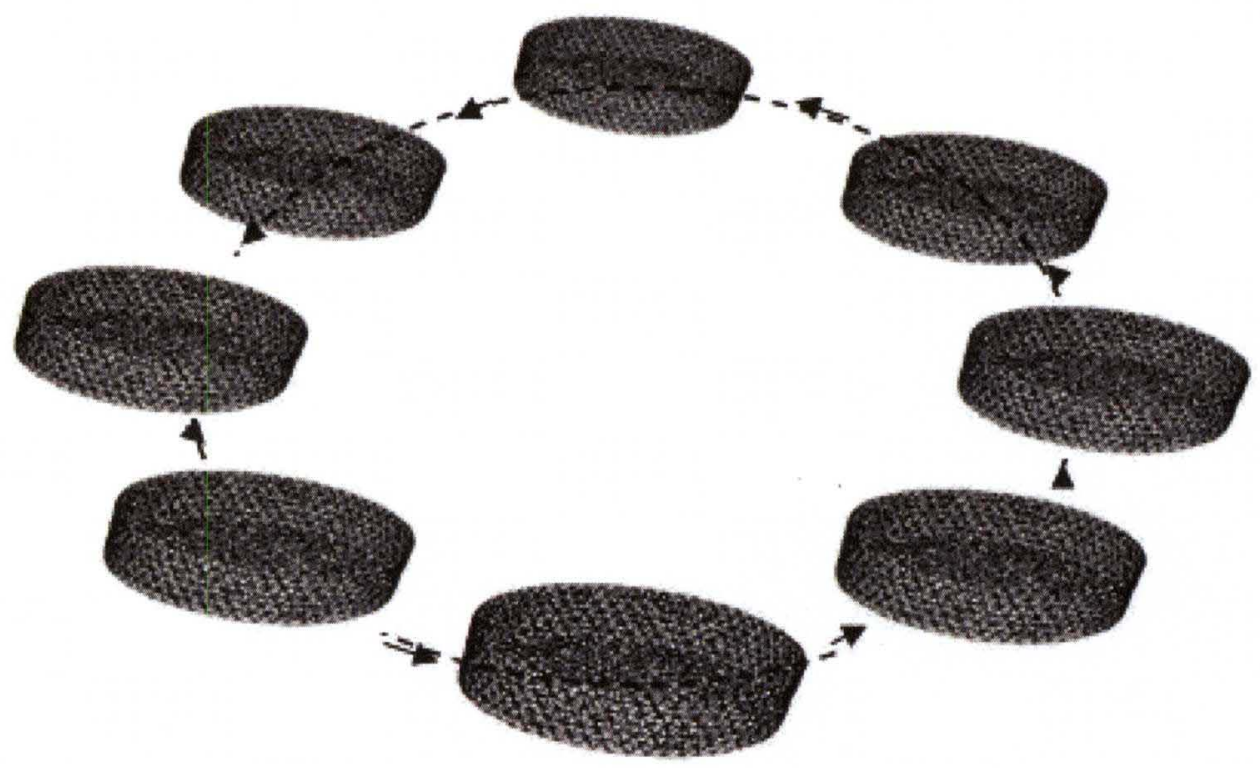

Figure 2.5. Model of orbital motion of a Petri dish

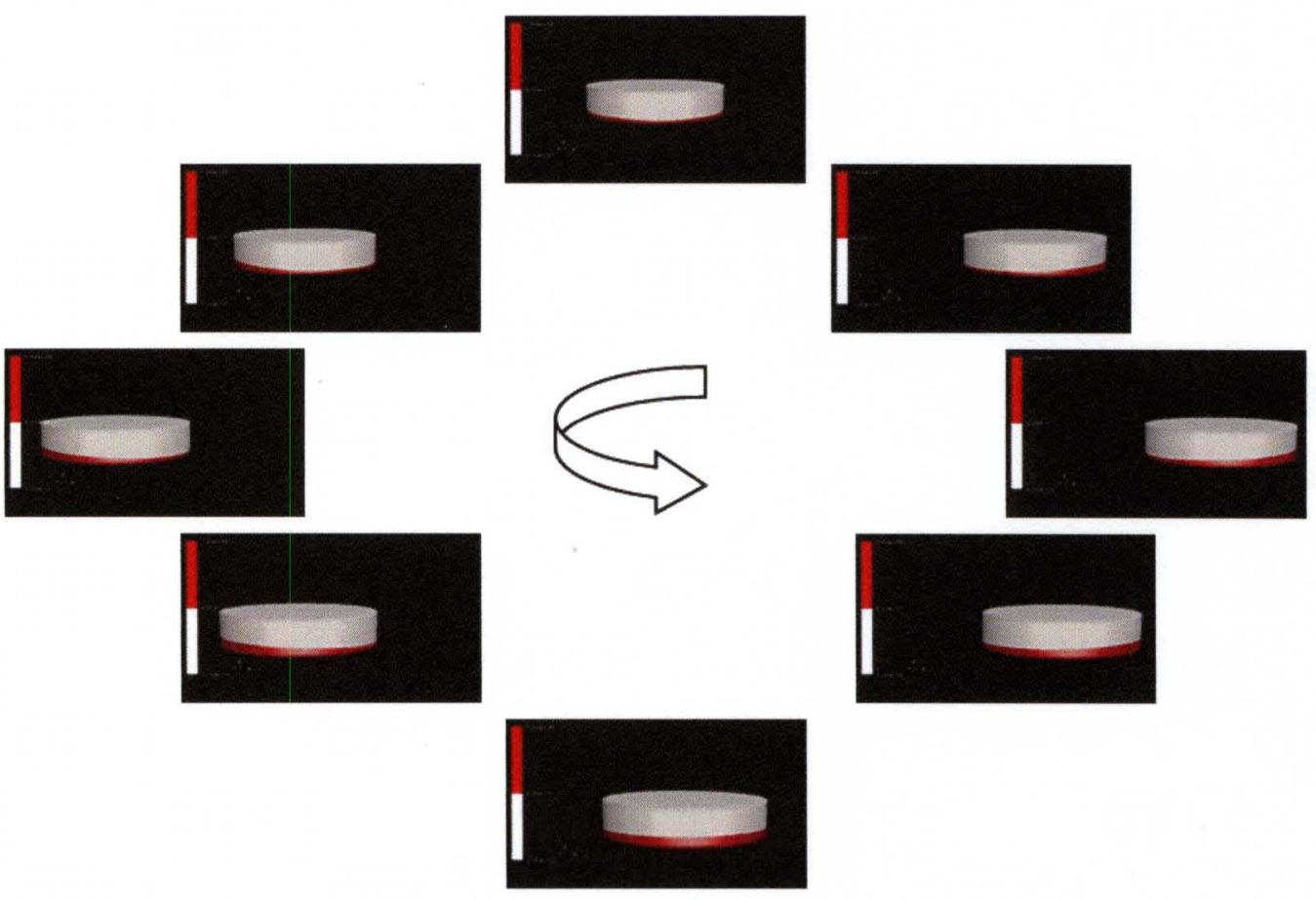

Figure 2.6. Example of liquid height profiles throughout one orbit. This is a multiphase model. The shallow red layer shows the liquid phase and white layer indicates air phase 


\subsubsection{Case set up procedure}

Dish dimensions, fluid properties, and orbital radius of the shaker were all defined equal to the same experimental parameters used by Dardik, et al. (36) since their work represented the limited experimental data that was available in the literature when this work began. A 3-D cylinder with a height of $20 \mathrm{~mm}$ and a radius of $17.5 \mathrm{~mm}$ was created in the pre-processor, GAMBIT, and then a mesh with 305,200 hexahedral computational cells was applied to the volume. This computational cell count was determined based on a previously defined optimization routine by Berson et al. (2008). The liquid in the dish was assigned a density of $997.3 \mathrm{~kg} \mathrm{~m}^{3}$ and a viscosity of 0.00101 $\mathrm{kg} \mathrm{m}$. The air above the liquid had a density of $1.225 \mathrm{~kg} / \mathrm{m}^{3}$ and a viscosity of $1.7894 \mathrm{E} 10^{-5} \mathrm{~kg} / \mathrm{m}-\mathrm{s}$. The initial height of liquid in the dish was $2 \mathrm{~mm}$.

WSS was calculated for six cases covering the following orbital speeds: 60,90 , $120,150,180$, and $210 \mathrm{rpm}$. Time step size was set as 0.001 seconds and the maximum number of time steps for each case was set so that the dish orbits at least 3.4 times, an amount previously determined to achieve oscillatory steady state in most cases Berson et al. (2008). Because fluid motion at any radial position in the dish is purely periodic, convergence can be evaluated by tracking WSS values at locations fixed relative to the orbital position of the dish. Convergence was defined as a variance of less than \pm .02 dyne $/ \mathrm{cm}^{2}$ between time steps, and was evaluated at the radial positions corresponding to the region of maximum WSS. The exact location of the maximum WSS region varies from case to case. 


\subsection{RESULTS AND DISCUSSION}

\subsubsection{Sample Simulation Result}

Figure 2.7 shows free surface contour and the corresponding WSS contour at an instant in time for the $120 \mathrm{rpm}$ case. While Equation 2.11 provided a single shear value for the entire surface, this CFD simulation exhibited significant variation of WSS over the bottom surface of the dish. The periodicity of the flow resulted in an oscillatory steady state solution which comprises a time-independent WSS contour, rotating at the speed of the orbiting motion of the orbiting dish. In Figure 2.7, shear stress magnitudes are low towards the center of the dish and there is a crescent shaped region near the edge of the dish representing higher ranges of shear magnitude. This is explained by Newton's law of viscosity (Equation 2.4). From Equation (2.4), the point of highest WSS will be expected at the point with the largest velocity gradient across the fluid height. The velocity difference between the fluid surface and bottom surface is greatest near the peak of the wave. The lowest fluid height occurred at the leading edge of the wave. 

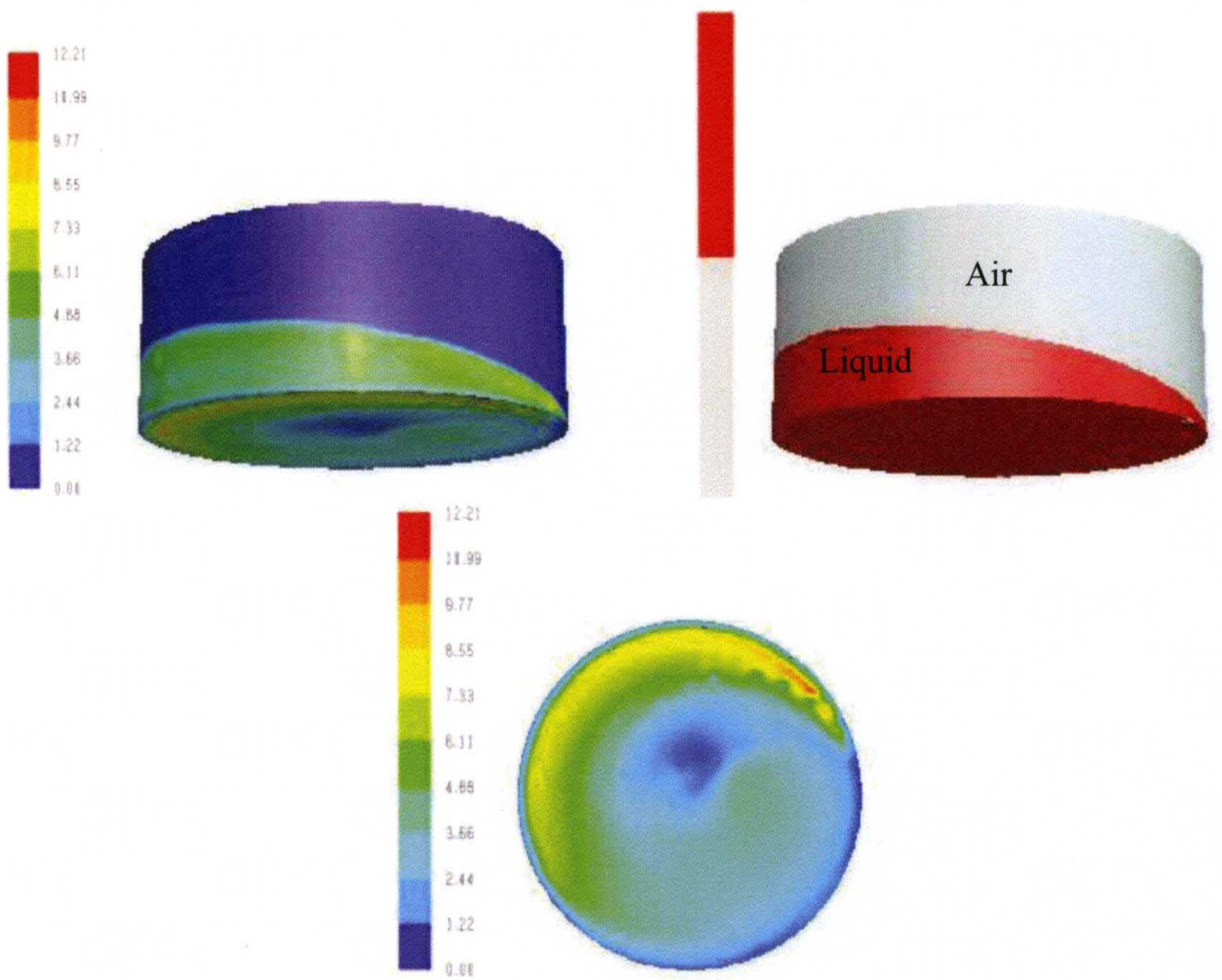

a) Side view of WSS contour b) Free surface contour c) Bottom Surface WSS Contour Figure 2.7. Resultant WSS magnitude $\left(\mathrm{dyne} / \mathrm{cm}^{2}\right)$ and free surface contours at $120 \mathrm{rpm}$.

\subsubsection{WSS as a Function of Radius and Orbital Time}

Figure 2.8 illustrates the resultant oscillatory WSS magnitudes on the bottom of the dish for the $210 \mathrm{rpm}$ case at three different radial locations $(16.4 \mathrm{~mm}, 12.8 \mathrm{~mm}$, and $4.25 \mathrm{~mm}$ ) from the center of the dish for one complete orbit. It was observed that WSS magnitudes were relatively constant throughout a complete orbit near the center of the dish. Further, with increase of radius, the amplitude of oscillation increased and near the side wall of the dish, the amplitude reached a peak in amplitude, as well as magnitude. 
Also there was a narrow band close to the side wall, indicating WSS decreased in response to the viscous layer on the side wall.

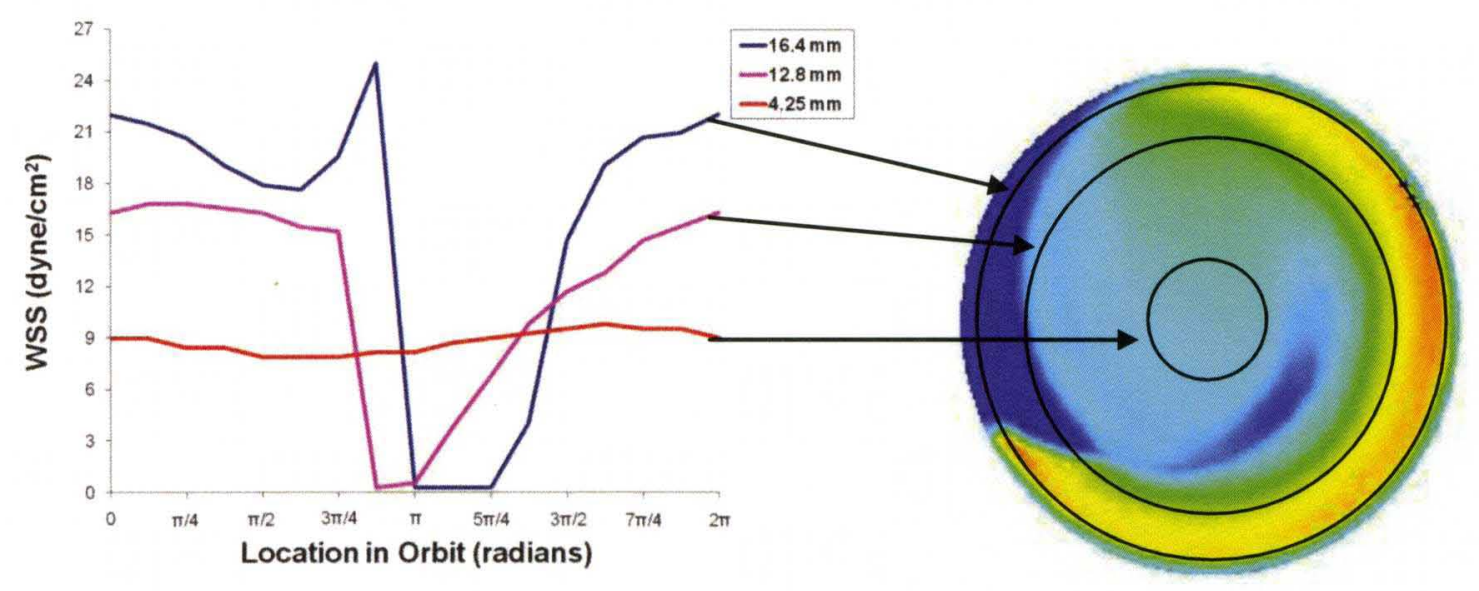

Figure 2.8. Resultant WSS magnitude for different radii at $210 \mathrm{rpm}$ throughout one orbit ( $2 \pi$ radians). The arrowheads indicate the radial position for each curve.

The overall non-uniformity of WSS across the dish in these simulations correlated with differences previously observed in cellular responses at different locations in the dish. As an example, Dardik, et al. (36) measured shear effects on various cellular responses in-vitro which include increased cell proliferation, apoptosis rates, increased intercellular molecule adhesion expression, reduced Akt phosphorylation, and reduced Eselectin down-regulation for endothelial cells seeded in the center compared to seeded in the periphery of a dish. 


\subsubsection{WSS as a Function of Orbital Velocity}

Figure 2.9 further describes the resultant oscillatory WSS magnitudes for low, medium, and high orbital speeds $(60,150$, and $210 \mathrm{rpm})$ at three different radial locations on the bottom of the dish for one complete orbital phase. For a particular radius, amplitudes of oscillation increased with increasing rpm, and were greater at increasing radial locations from the center of the dish. From the figure, it was evident that WSS was nearly constant at about $0.4 \mathrm{dyne} / \mathrm{cm}^{2}$ at all three radii at low orbital speed $(60 \mathrm{rpm})$. At150 rpm, the peak WSS was $\sim 6.5 \mathrm{dyne} / \mathrm{cm}^{2}$ at $4.25 \mathrm{~mm}, \sim 9.5 \mathrm{dyne} / \mathrm{cm}^{2}$ at $12.8 \mathrm{~mm}$, and $\sim 16$ dyne $/ \mathrm{cm}^{2}$ at $16.4 \mathrm{~mm}$. At $210 \mathrm{rpm}$, the peak WSS was $\sim 9.5 \mathrm{dyne} / \mathrm{cm}^{2}$ at 4.25 $\mathrm{mm}, \sim 17 \mathrm{dyne} / \mathrm{cm}^{2}$ at $12.8 \mathrm{~cm}$, and $\sim 25 \mathrm{dyne} / \mathrm{cm}^{2}$ at $16.4 \mathrm{~mm}$. 


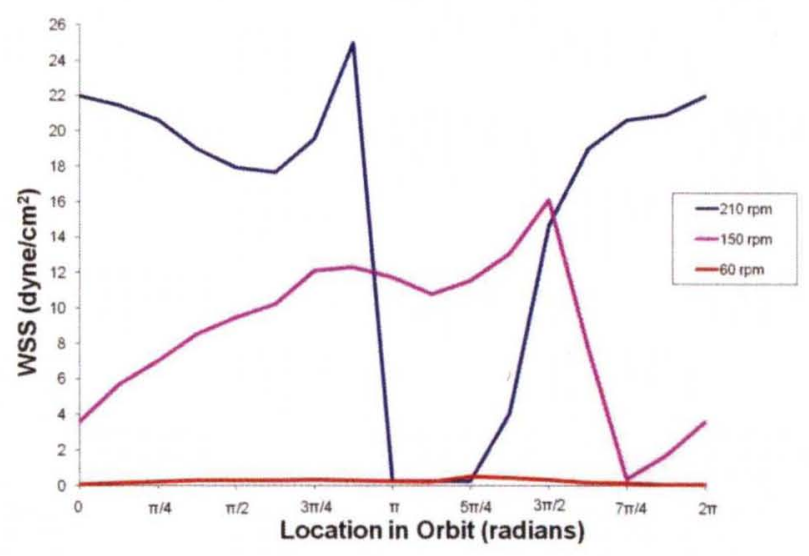

Figure 2.9 -a

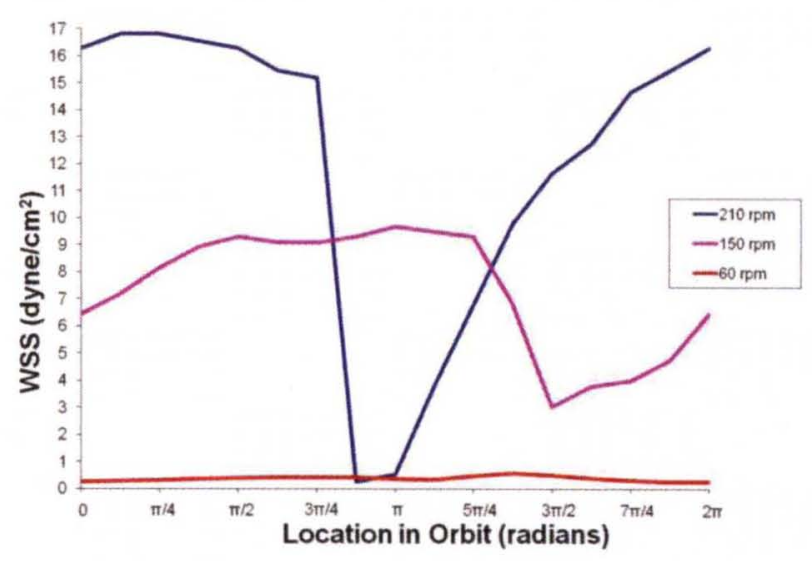

Figure 2.9-b

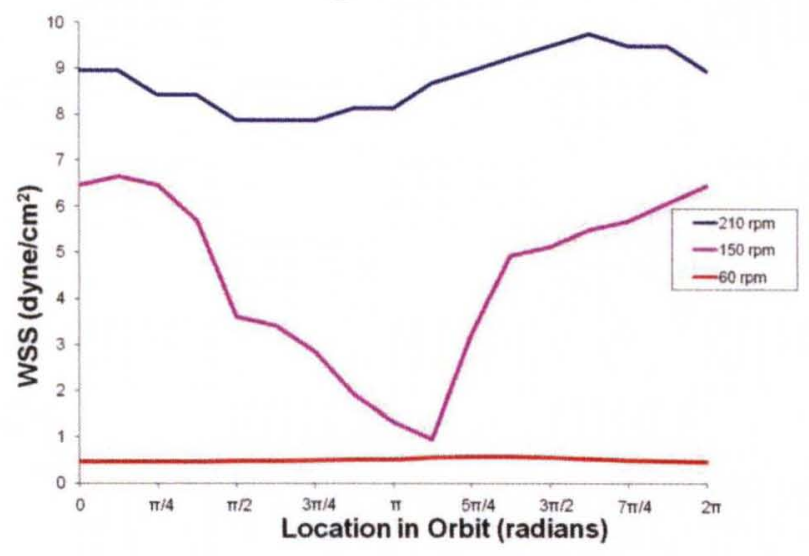

Figure 2.9 - c

Figure 2.9. Resultant WSS magnitude as a function of radius and orbital velocity at radial locations of $16.4 \mathrm{~mm}(\mathrm{a}), 12.8 \mathrm{~mm}(\mathrm{~b})$, and $4.25 \mathrm{~mm}$ (c) throughout one orbit. 


\subsubsection{Comparison with Analytical Solution}

As explained above, the Stokes second problem solution (Equation 2.11) provided an estimate for a steady, constant resultant WSS magnitude for flow in the Petri-dish. Since the analytical solution assumes no wall effects and the CFD analysis illustrated that there was near constant steady shear magnitudes near the center of the dish, WSS magnitudes at the center of the dish at different orbital speeds $(60 \mathrm{rpm}, 90 \mathrm{rpm}, 120 \mathrm{rpm}$, $150 \mathrm{rpm}$, and $210 \mathrm{rpm}$ ) were selected from the computational model and compared with the analytical solution or Stokes $2^{\text {nd }}$ problem solution (Equation 2.11) in Figure 2.10. (A dimensional analysis is undertaken in Chapter Three and values of dimensionless parameters for the cases in Figure 2.10 appear in Appendix D.) Further, the area average WSS magnitude from the computational model was compared with the analytical solution. Analytically determined WSS values compare reasonably well with the computationally determined WSS magnitudes near the center of the dish throughout the range, but were $0.99 \pm 0.42 \mathrm{dyne} / \mathrm{cm}^{2}$ higher. At 60 and $90 \mathrm{rpm}$, the center WSS magnitudes matched the area average WSS values. At 120 and higher, the area average WSS magnitudes are greater than the center WSS values, the difference may be caused by wall effects. The area average WSS magnitudes were also slightly higher than values from the Stokes second problem solution in this range. The area average WSS magnitudes were $0.10 \pm 1.02 \mathrm{dyne} / \mathrm{cm}^{2}$ higher than the Stokes second problem solution over the full range of orbital speeds. 


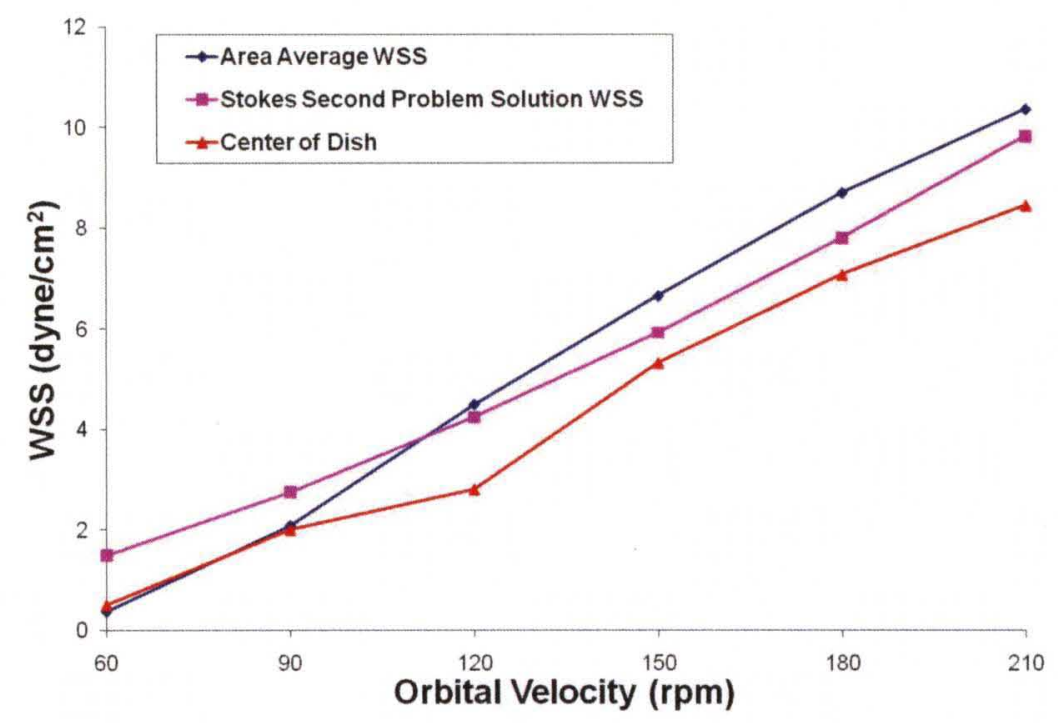

Figure 2.10. Comparison of area average WSS magnitude from the computational model with values from the analytical solution as the function of orbital speeds.

\subsubsection{Comparison with Experimental Oscillatory WSS}

The model was next compared to experimental WSS values reported in the literature [Dardik, et al. (36)]. They used a one-dimensional optical Doppler velocimetry probe to measure wall shear stress and, in this case, they measured the tangential component. One important limitation of using Doppler velocimetry was that the probe cannot distinguish between positive and negative Doppler shifts, therefore the calculated WSS values were all positive. As a result, all the scattered plots of Doppler shifts were interpreted as positive in that study (36) which were generated from the ultrasonic velocity probe for a single case $(210 \mathrm{rpm})$ throughout several orbits. The scatter plot data was then converted to WSS by Equation (24) and presented here for comparison to computationally determined shear. Figure 2.11 and 2.12 showed the comparison of the computationally determined oscillating tangential WSS magnitude with experimentally 
determined WSS for one orbit. Computationally determined WSS was superimposed over the WSS scattered plots. The scatter plots encompassed 1400 orbits for the radial location of $12 \mathrm{~mm}$ and 1183 orbits for the radial location of $1 \mathrm{~mm}$ from center of the dish. The sampling rates for these measurements provided one data point every 12.6 orbits at 1 $\mathrm{mm}$ from the center of the dish and one data point every 17.7 orbits at $12 \mathrm{~mm}$ from the center of the dish.

In Figure 11, the experimental minimum and maximum WSS at $12 \mathrm{~mm}$ from the center of the dish were 6 and 16 dyne $/ \mathrm{cm}^{2}$, respectively, whereas WSS generated from the computational model ranged from 0.5 to $14 \mathrm{dyne} / \mathrm{cm}^{2}$. This compares reasonably well in the upper range, but not as well in the lower range.

One reason that they do not match well in the lower range might be that the experimental WSS values were collected in a region where the model predicts a sharp gradient in WSS in the radial direction. For instance at $210 \mathrm{rpm}$, using the simulated gradient at $12 \mathrm{~mm}$, a $8 \%(1 \mathrm{~mm})$ error in measured radial position could result in a $16 \%$ (1.9 dyn $\left./ \mathrm{cm}^{\wedge} 2\right)$ error in measured WSS. With such a sparse sampling rate, it was possible that the lowest value actually present in the experiment was not recorded during the sampling time. Also, the experimental probe might not able to pick up very low values which occur in a shallow liquid layer over the probe. At these times, the number of $\mathrm{TiO}_{2}$ particles over the Doppler probe would be small and scattering of the signal by the free surface would be strong enough that perhaps the signal could not be distinguished from noise and hence was not recorded by the experimental software.

Figure 2.12 shows the comparison of the measured minimum and maximum tangential WSS magnitudes (computational and experimental) at $1 \mathrm{~mm}$ from the center of 
the dish. The experimentally determined minimum WSS and maximum WSS were 3 and 7 dyne $/ \mathrm{cm}^{2}$, respectively, whereas the computational WSS magnitudes varied from 0.5 to $8.5 \mathrm{dyne} / \mathrm{cm}^{2}$. The computational WSS value from 0 to $\pi / 8$ and $5 \pi / 4$ to $2 \pi$, which were originally negative, have been inverted to the positive per the discussion above. Those two values (computational and experimental) did not match quite as well in Figure 2.12 as those from Figure 2.11. A potential possible reason may be the sparse sampling which may cause the missing of both low and high WSS values. Further, there may be an error in locating the probe measurement volume, which might reduce the ranges of average WSS values, as well as WSS amplitude. 


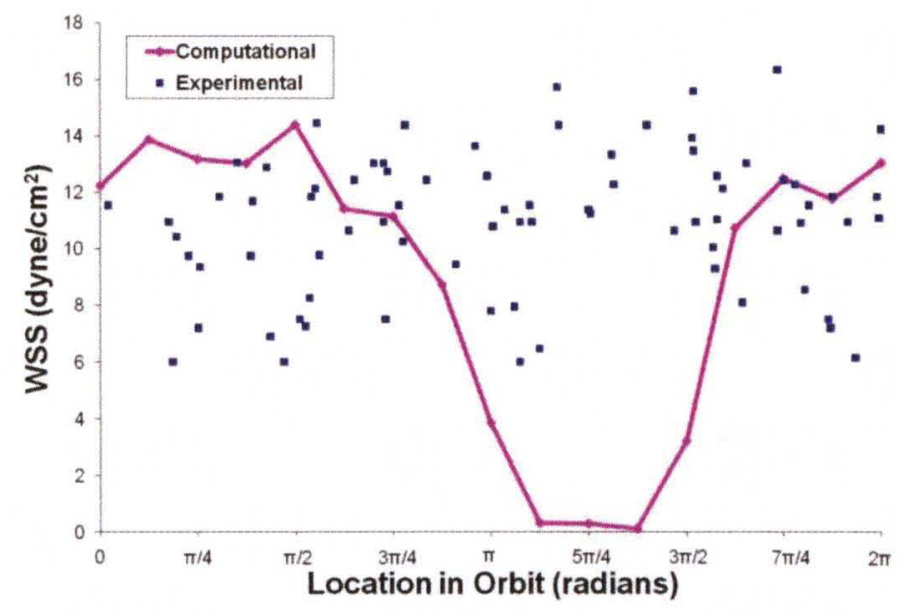

Figure 2.11. Comparison of computational and experimental tangential WSS magnitudes at $12 \mathrm{~mm}$ from center of dish and $210 \mathrm{rpm}$.

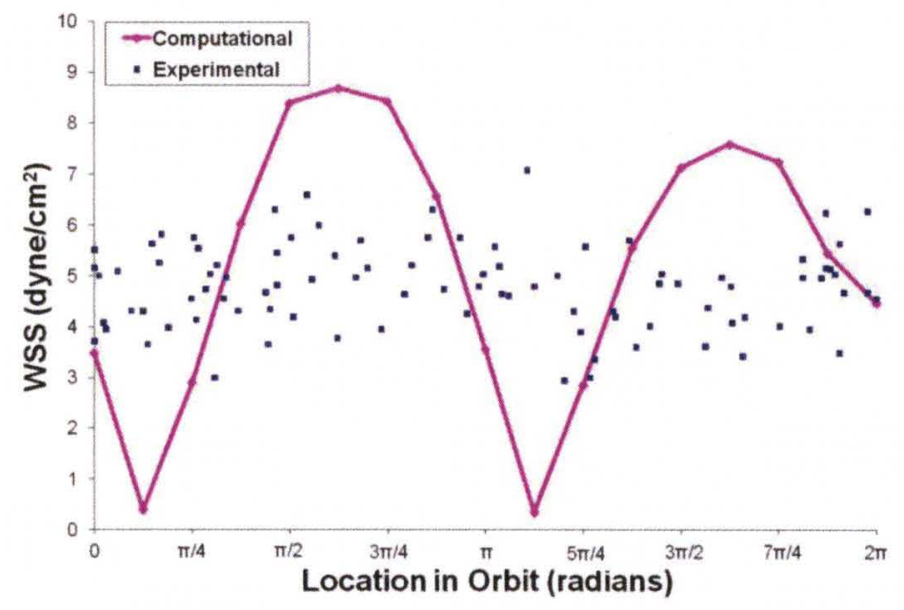

Figure 2.12. Comparison of computational and experimental WSS magnitudes at $1 \mathrm{~mm}$ from center of dish and $210 \mathrm{rpm}$. 


\subsubsection{CONCLUSIONS}

A computational model involving a simulated motion of fluid in a dish on an orbiting shaker apparatus was developed which provided a new method for determining WSS on the bottom surface of the dish with complete spatial and temporal resolution. The results confirmed that WSS values from the computational simulation are a more useful representation of shear, since they are not limited to a constant value, rather they give more realistic oscillatory un-steady state shear values. As a result, the computational model allows cellular responses to be examined under oscillatory WSS values, which provides a more accurate representation of the actual shear experienced by anchored cells. The model was reasonably well validated by both an analytical solution and experimental WSS values. Steep WSS gradients were found in the computational results that suggest that measured WSS is sensitive to probe location. The model significantly enhances the usefulness of simple shaker apparatus in the study of hemodynamic effects on endothelial and other anchored cell cultures by providing WSS as a function of space and time over all dish locations. 


\section{CHAPTER THREE}

\section{DIMENSIONAL ANALYSIS OF FLOW IN ORBITING PETRI-DISHES}

\subsection{INTRODUCTION AND BACKGROUND}

\subsubsection{Brief description about the dimensionless parameters}

\section{Stokes Number:}

It is the ratio of inertial forces from the dish versus viscous forces from the fluid

$$
S t=H^{*} \sqrt{\frac{\omega}{v}}
$$

For low Stokes Number, the fluid motion will be viscous dominated. For high Stokes Number, the flow will be inertially dominated, and there will be a phase lag behind the motion of the cylinder.

\section{Froude Number:}

It is the ratio of convective and gravitational forces.

$$
F r=\frac{u}{\sqrt{g^{*} H}}
$$


In an orbiting dish, Froude transition can be determined from visual inspection; the magnitude of the Froude Number determines the steepness of the leading edge of the wave.

\section{Slope Ratio:}

Slope is a measure of the steepness of a straight line and it is defined as:

$$
m=\frac{\Delta Y}{\Delta X}
$$

For the system of interest, it is defined as the ratio of the quasi-steady free surface slope and the aspect ratio of the fluid.

$$
S l=\frac{R^{*} a^{*} \omega^{2}}{g^{*} H}
$$

For an orbiting dish, at low slope ratio, there will be a smooth downward slope of the wave. The fluid is considered to have a high Slope Ratio when concavity is present. At large Slope Ratio, the slope of the trailing edge of the wave will become extremely high and that will eventually dry a part of the base of the cylindrical dish. A gravitational force here tries to prevent the drying by extending the trailing edge of the wave. 


\subsection{METHODOLOGY}

\subsubsection{Development of dimensionless parameters}

Fluid forces inside an orbiting dish are the combination of seven system properties including: radius of orbit, orbital speed, dish diameter, viscosity, fluid height, density, and gravity. A dish labeled with these seven parameters is seen in Figure 3.1.

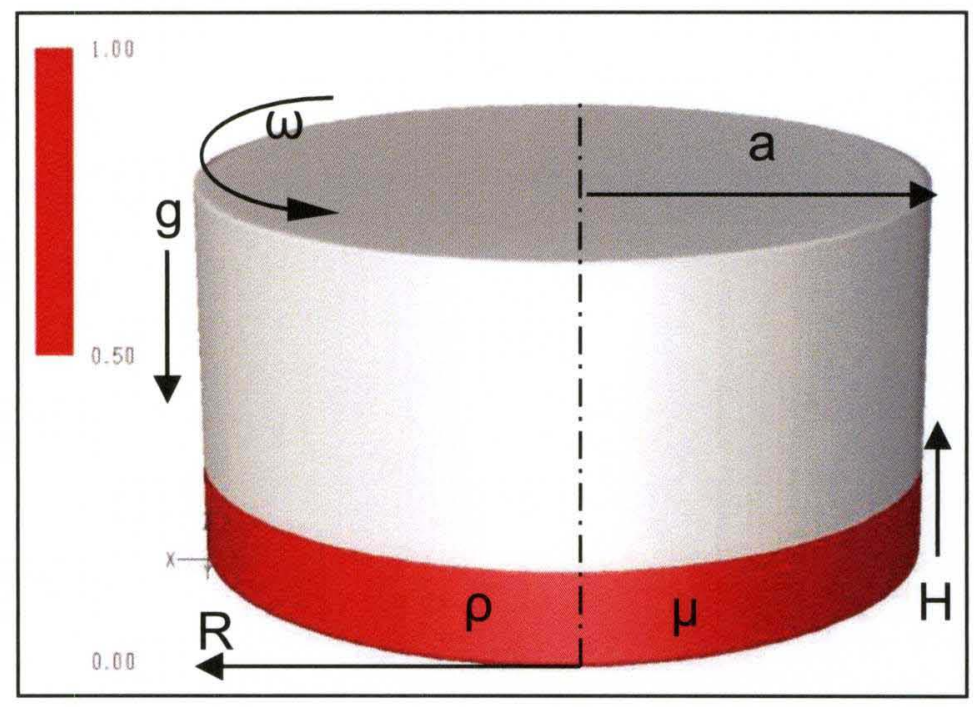

Figure 3.1. Dish with seven parameters

The nomenclature in the figure is: $\mathrm{a}=$ cylinder radius, $\mathrm{H}=$ static height of the fluid in the cylinder, $\mathrm{R}=$ radius of orbit, $\omega=$ angular velocity of orbit, $\mathrm{g}=$ acceleration of gravity, $\mu=$ fluid viscosity, $\rho=$ fluid density.

If cases were set up to view the effects each parameter at a low and high value, the total number of cases to view each combination of parameters would be 840 . This would require an unnecessarily vast computational effort. It was therefore desirable to reduce the number of variables to decrease the computational effort. Rance, et al. (72) showed that dimensional analysis can reduce the seven system properties to four 
dimensionless parameters, which are Slope ratio, Froude number, Stokes number and Reynolds number. Flow in the orbital shaker apparatus was limited here to laminar flow. Therefore, a constant laminar Reynolds number of 100 was used to reduce the number of parameters to three. Thus, using just three parameters for low and high values, the effects of seven system properties on fluid flow can be reduced to only 27 cases (represented by black dots in Figure 3.2). This study encompasses (1) how fluid behaves at low and high values for each of the parameters represented by the red lines in Figure 3.2 and (2) finding where the transition from high to low for each of these values occurs. A collaborative study was done to analyze those cases that include the 12 dots connected by the blue lines in Figure 3.2.

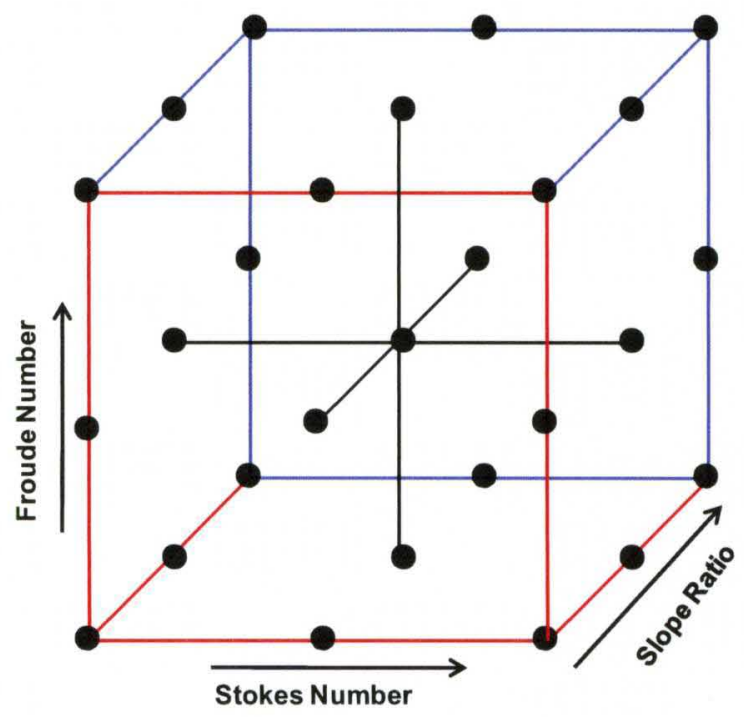

Figure 3.2. Cube of Dimensionless parameters 


\subsubsection{Free surface analysis of dimensional parameters in determining transition points}

\section{Froude transition:}

Froude transition can be determined by free surface analysis as shown in Figure 3.3. For low values, the slope of the leading edge is similar to the slope of the rest of the wave. The slope of the leading edge becomes greater when Froude Number increases towards the transition (Figure 3.3A). At the transition, there will be a rippled effect on the leading edge of the wave. At high Froude numbers after the transition, the fluid wave becomes unsteady and develops into an overshooting wave (Figure 3.3B).

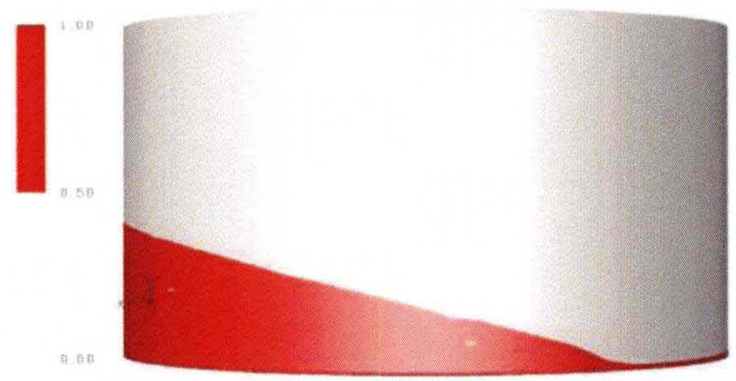

Figure 3.3A. Low Froude Number

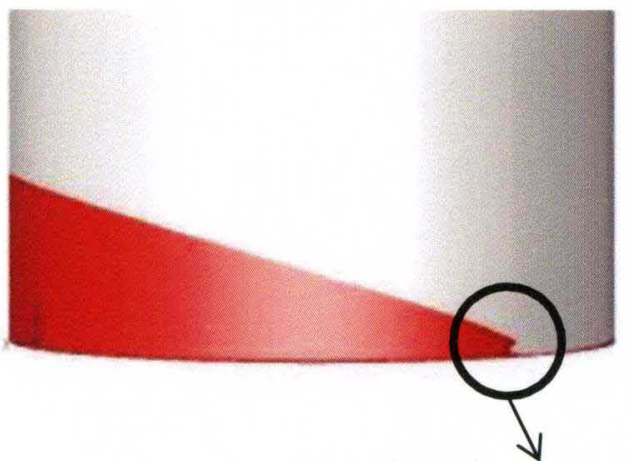

Overshooting effect

Figure 3.3. Examples of Froude effect.

\section{Slope Ratio transition:}

The Slope Ratio transition can also be determined from visual inspection of the free surface contours (Figure 3.4). The trailing edge of the wave shows a smooth slope downward for low slope ratio (Figure 3.4A). For high Slope Ratio (Figure 3.4B), the 
wave shows a large visible concavity on its trailing edge. The transition is at the point where the trailing edge of the wave just becomes concave.

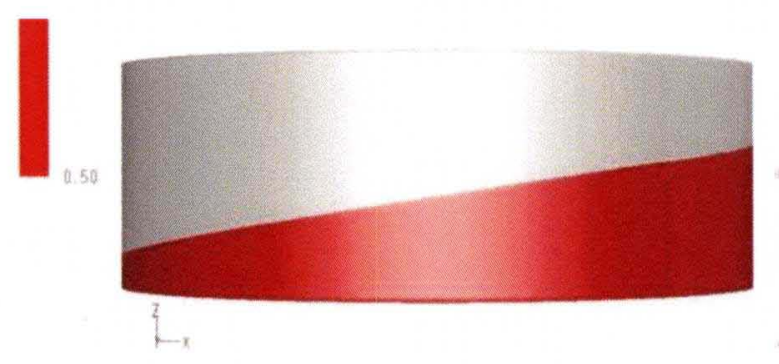

Figure 3.4A. Low Slope Ratio

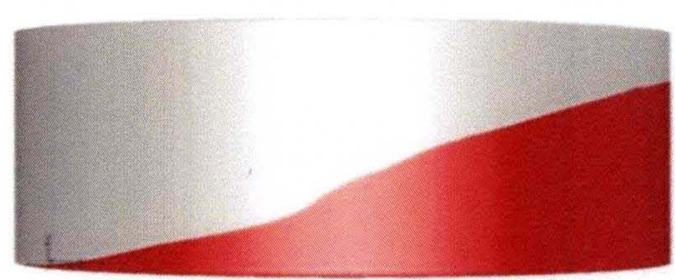

Figure 3.4B. High Slope Ratio

Figure 3.4. Examples of Slope Ratio effect.

\section{Stokes Number Transition:}

In an orbiting motion, the fluid wave will be in phase with the cylinder's motion when the Stokes number is below the transition (Figure 3.5A), whereas above the transition, the peak of the wave will lag behind the location of the cylinder (Figure 3.5B). The degree of phase lag can be calculated by the following equation.

$$
\text { Degree of phase lag }=\frac{360}{N-1} * n
$$

where,

$\mathrm{n}=$ the number of cells counted between the center line of the dish and the peak of the fluid, $\mathrm{N}=$ Number of total cells. 


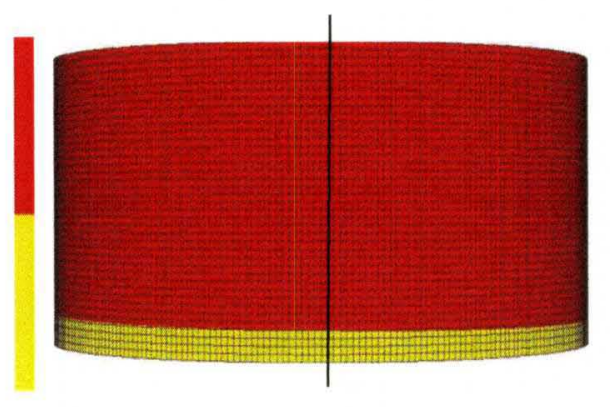

Figure 3.5A. Low Stokes Number

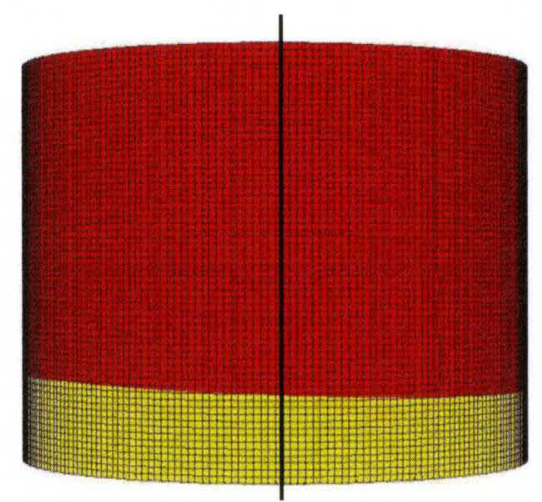

Figure 3.5B. High Stokes Number

Figure 3.5. Example of Stokes Number Effects

\subsubsection{Work Plan}

Six transitions from low to high will be found for the conditions defined by the red lines in Figure 3.2 and also described in Table 3.1. To determine each transition, several cases for each of the parameters will be run from expected low to high values by keeping the other two parameters constant. Once initial results of each dimensionless parameter listed in Table 3.1 are determined, an extra set of cases to the next decimal place will be developed to more precisely define the transition point. The free surface plots and shear contours of each of the cases will be analyzed to determine the transition point. Once the transition is determined, WSS trends will be analyzed below, at, and above the transition. In addition, plots of tangential WSS vs. radial WSS will be analyzed to understand WSS characteristic for values below, above, and at the transition of each parameter. Furthermore, the transition points, previously determined by free surface plots, may be verified by analyzing trends in these plots. 
Table 3.1. Initial dimensionless parameter values to be tested for determining transitions

\begin{tabular}{|c|c|c|c|c|c|}
\hline \begin{tabular}{l}
\multicolumn{1}{c}{ SET1 } \\
Varying Slope \\
Ratio \\
(Froude No:0.1 \\
Stokes No:1.0)
\end{tabular} & \begin{tabular}{l}
\multicolumn{1}{c}{ SET2 } \\
Varying Slope \\
Ratio \\
(Froude No:0.1 \\
Stokes No:10)
\end{tabular} & \begin{tabular}{l}
\multicolumn{1}{c}{ SET3 } \\
Varying Froude \\
Number \\
(Slope \\
Ratio:0.1 \\
Stokes No: \\
1.0 )
\end{tabular} & \begin{tabular}{l}
\multicolumn{1}{c}{ SET4 } \\
Varying \\
Froude Number \\
(Slope \\
Ratio:0.1 \\
Stokes No: \\
10)
\end{tabular} & \begin{tabular}{l}
\multicolumn{1}{c}{ SET5 } \\
Varying \\
Stokes \\
Number \\
(Slope \\
Ratio:0.1 \\
Froude No: \\
0.1 ) \\
\end{tabular} & \begin{tabular}{l}
\multicolumn{1}{c}{ SET6 } \\
Varying Stokes \\
Number \\
(Slope Ratio: \\
0.1 \\
Froude No: \\
1.2 )
\end{tabular} \\
\hline 0.1 & 0.1 & 0.1 & 0.1 & 1 & 1 \\
\hline 0.2 & 0.2 & 0.2 & 0.2 & 2 & 2 \\
\hline 0.3 & 0.3 & 0.3 & 0.3 & 3 & 3 \\
\hline 0.4 & 0.4 & 0.4 & 0.4 & 4 & 4 \\
\hline 0.5 & 0.5 & 0.5 & 0.5 & 5 & 5 \\
\hline 0.6 & 0.6 & 0.6 & 0.6 & 6 & 6 \\
\hline 0.8 & 0.8 & 0.8 & 0.8 & 7 & 7 \\
\hline 1 & 1 & 1 & 1 & 8 & 8 \\
\hline
\end{tabular}




\subsection{RESULTS \& DISCUSSION}

\subsubsection{WSS analysis as a function of Slope ratio at constant Stokes Number and constant Froude Number}

Liquid properties, dish dimensions, and orbital speed of this set are tabulated in Table 3.2A. From the free surface analysis of the initial cases, the transition point appeared to fall in between a slope ratio of 0.4 and 0.5 . Four more cases between these values were run (Table 3.2B) and from the free surface analysis, the transition point was found to be at a Slope ratio of 0.48 .

Table 3.2A. Initial cases for Set 1 and related system properties

\begin{tabular}{|c|c|c|c|c|c|c|c|c|c|c|}
\hline Cases & Gravity & $\begin{array}{c}\text { Radius } \\
\text { of } \\
\text { orbit } \\
\text { cm }\end{array}$ & $\begin{array}{l}\text { Radius } \\
\text { of dish }\end{array}$ & $\begin{array}{l}\text { Mean } \\
\text { height } \\
\text { of } \\
\text { fluid } \\
\mathrm{Cm}\end{array}$ & $\begin{array}{c}\text { Orbital } \\
\text { speed }\end{array}$ & $\begin{array}{l}\text { Kinematic } \\
\text { viscosity }\end{array}$ & $\begin{array}{l}\text { Slope } \\
\text { ratio }\end{array}$ & $\begin{array}{c}\text { Froude } \\
\text { No. }\end{array}$ & $\begin{array}{c}\text { Stokes } \\
\text { No. }\end{array}$ & $\begin{array}{c}\text { Reynolds } \\
\text { No. }\end{array}$ \\
\hline 1 & 9.8 & 1.2 & 1.2000 & 0.0120 & 0.9037 & $1.3013 \mathrm{E}-08$ & 0.1 & 0.1 & 1 & 100 \\
\hline 2 & 9.8 & 1.2 & 0.6000 & 0.0060 & 1.2780 & $4.6009 \mathrm{E}-09$ & 0.2 & 0.1 & 1 & 100 \\
\hline 3 & 9.8 & 1.2 & 0.4000 & 0.0040 & 1.5652 & 2.5044E-09 & 0.3 & 0.1 & 1 & 100 \\
\hline 4 & 9.8 & 1.2 & 0.3000 & 0.0030 & 1.8074 & $1.6267 \mathrm{E}-09$ & 0.4 & 0.1 & 1 & 100 \\
\hline 5 & 9.8 & 1.2 & 0.2400 & 0.0024 & 2.0207 & $1.1639 \mathrm{E}-09$ & 0.5 & 0.1 & 1 & 100 \\
\hline 6 & 9.8 & 1.2 & 0.2000 & 0.0020 & 2.2136 & $8.8544 \mathrm{E}-10$ & 0.6 & 0.1 & 1 & 100 \\
\hline 7 & 9.8 & 1.2 & 0.1500 & 0.0015 & 2.5560 & $5.7511 \mathrm{E}-10$ & 0.8 & 0.1 & 1 & 100 \\
\hline 8 & 9.8 & 1.2 & 0.1200 & 0.0012 & $\mathbf{2 . 8 5 7 7}$ & $4.1151 \mathrm{E}-10$ & 1 & 0.1 & 1 & 100 \\
\hline 9 & 9.8 & 1.2 & 0.0600 & 0.0006 & 4.0415 & $1.4549 \mathrm{E}-10$ & 2 & 0.1 & 1 & 100 \\
\hline
\end{tabular}

Table 3.2B. Transition cases for Set 1 and related system properties

\begin{tabular}{|c|c|c|c|c|c|c|c|c|c|c|}
\hline Cases & Gravity & $\begin{array}{c}\text { Radius } \\
\text { of } \\
\text { orbit } \\
\mathrm{cm} \\
\end{array}$ & $\begin{array}{l}\text { Radius } \\
\text { of dish }\end{array}$ & $\begin{array}{c}\text { Mean } \\
\text { height } \\
\text { of } \\
\text { fluid } \\
\mathrm{Cm}\end{array}$ & $\begin{array}{c}\text { Orbital } \\
\text { speed }\end{array}$ & $\begin{array}{c}\text { Kinematic } \\
\text { viscosity } \\
\mathrm{m}^{2} / \mathrm{s} \\
\end{array}$ & $\begin{array}{l}\text { Slope } \\
\text { ratio }\end{array}$ & $\begin{array}{c}\text { Froude } \\
\text { No. }\end{array}$ & $\begin{array}{c}\text { Stokes } \\
\text { No. }\end{array}$ & $\begin{array}{c}\text { Reynolds } \\
\text { No. }\end{array}$ \\
\hline 1 & 9.8 & 1.2 & 0.2857 & 0.0029 & 1.8520 & 1.5119E-09 & 0.42 & 0.1 & 1 & 100 \\
\hline 2 & 9.8 & 1.2 & 0.2727 & 0.0027 & 1.8956 & $1.4100 \mathrm{E}-09$ & 0.44 & 0.1 & 1 & 100 \\
\hline 3 & 9.8 & 1.2 & 0.2609 & 0.0026 & 1.9382 & $1.3190 \mathrm{E}-09$ & 0.46 & 0.1 & 1 & 100 \\
\hline 4 & 9.8 & 1.2 & 0.2500 & 0.0025 & 1.9799 & $1.2374 E-09$ & 0.48 & 0.1 & 1 & 100 \\
\hline
\end{tabular}


Figure 3.6 shows the resultant WSS contours (on two scales) below, at, and above the Slope ratio transition for constant low Froude Number of 0.1 and low Stokes Number of 1.0. Below the transition WSS is of low magnitude and is relatively uniform. Above the transition: the contour has two distinct regions, a region of low uniform shear and a region of higher shear. The boundary between these two regions is where the fluid must intersect the dish because of the high slope. The contour at the transition is similar but with higher shear and there is one area of localized increased shear, which is likely where the liquid intersects the bottom of the dish on the leading edge of the wave.

Figure 3.7 illustrates the plots of radial and tangential WSS components for the three different regions (below the transition at Slope ratio of 0.1 , at the transition at Slope ratio of 0.48 , and after the transition at Slope ratio of 1.0$)$ at four radial locations $(20 \%$, $40 \%, 60 \%$, and $80 \%$ ) on the bottom surface of the dish. Radial and tangential components of WSS at 32 equally spaced points around one complete orbit were extracted from each contour at each radial location. A MATLAB (Appendix A4) program was used to find the exact node values for the two shear components for each case. Figure 3.7A shows the tangential vs. radial WSS plots for the three Slope ratios $(0.1,0.48$, and 1.0$)$ at $20 \%$ radial location. Figures $3.7 \mathrm{~B}, 3.7 \mathrm{C}$, and $3.7 \mathrm{D}$ show the corresponding plots at $40 \%, 60 \%$, and $80 \%$ radial locations, respectively. Below the transition (Slope ratio of 0.1 ) the WSS distributions are evenly distributed between radial and tangential components at all radial locations. At the transition and above, the plots are generally irregular shaped especially away from the center, but seem to be fairly symmetric regarding the distribution between tangential and radial components. The "flattening" of the plots in the first and second quadrants coincides with the two distinct 
regions in the contours of Figure 3.6 where about half of the surface is of very low and uniform shear due to the high slope ratio. The magnitudes gradually increase with increasing radii at and above the transition. Interestingly, the magnitude of WSS components were higher at the transition point than either above or below the transition, which is likely due less fluid coverage of the surface for the case above the transition . 
A

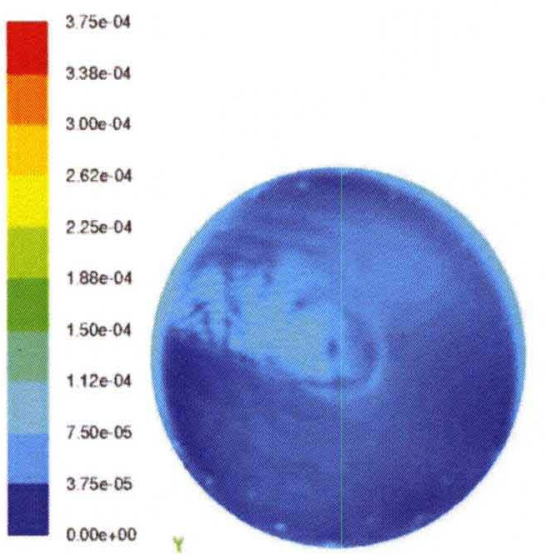

$\mathrm{Pa}$

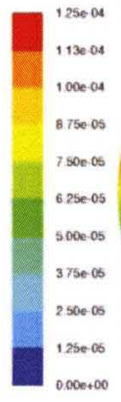

$\mathrm{Pa}$
B
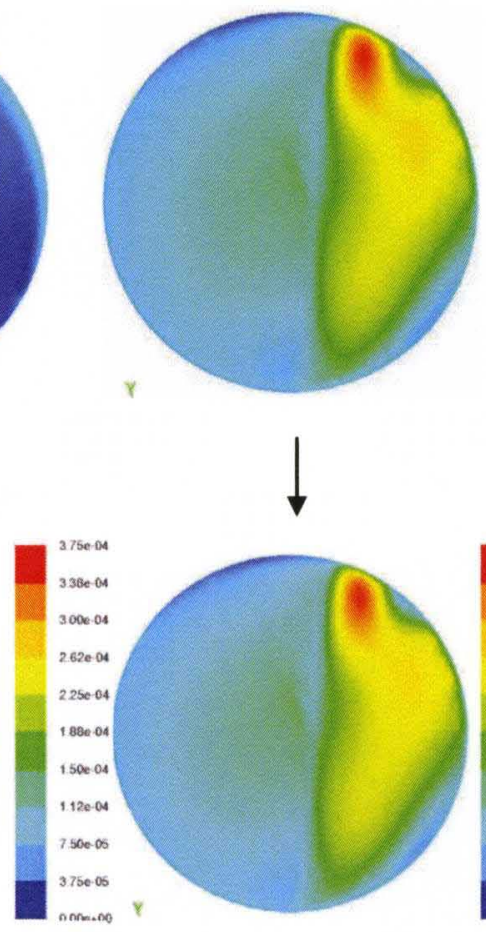

$\mathrm{Pa}$
$\mathrm{C}$
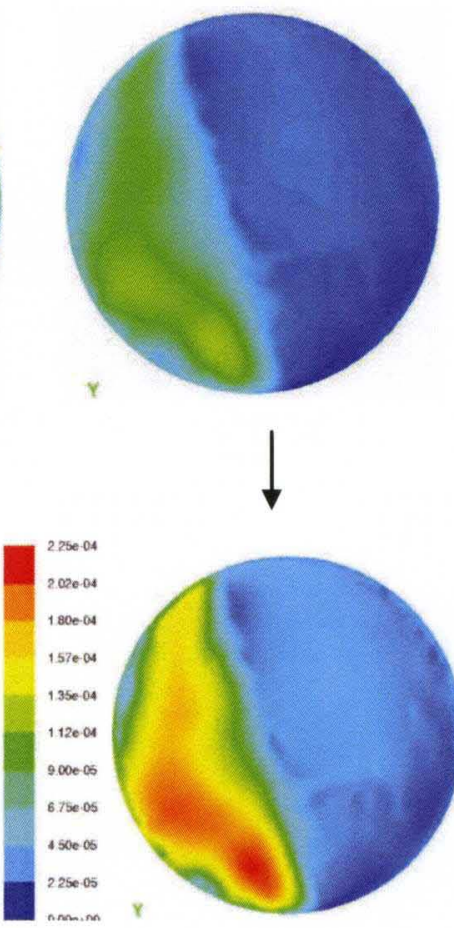

$\mathrm{Pa}$

Figure 3.6. Resultant WSS contours below, at, and above the Slope ratio transition for constant low Froude Number of 0.1 and low Stokes Number of 1.0. The contours are shown with a common scale in the first row and with individual maximum scales in the second row. Figure 3.6A is the resultant WSS contour at a Slope ratio of 0.1 (which is well below the fluid transition); Figure 3.6B is the resultant WSS contour at a Slope ratio of 0.48 (at the transition); Figure 3.6C is the resultant WSS contour at a Slope ratio of 1.0 (well above the transition). 


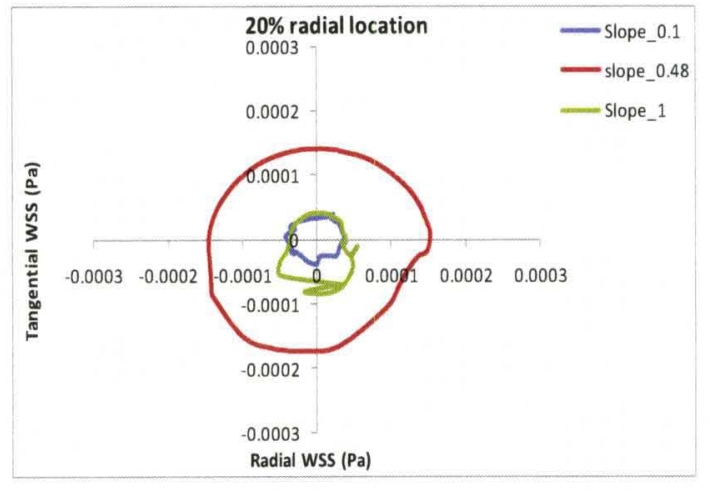

$\mathrm{C}$

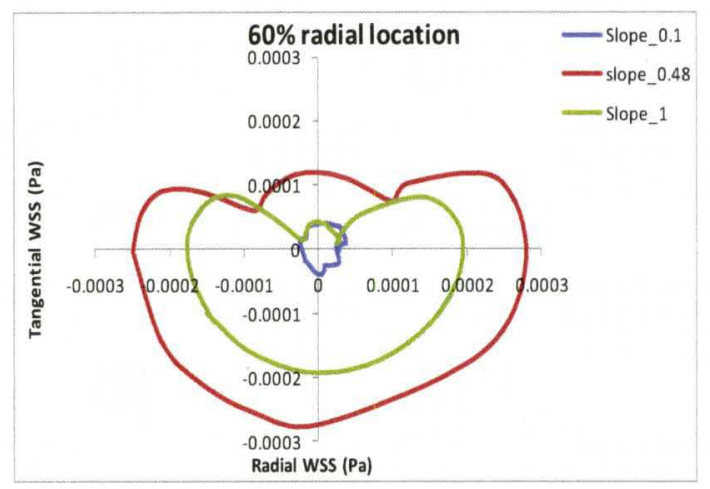

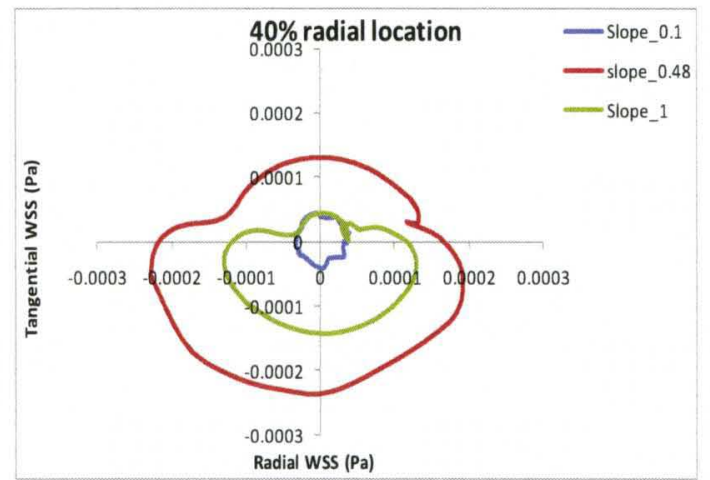

D

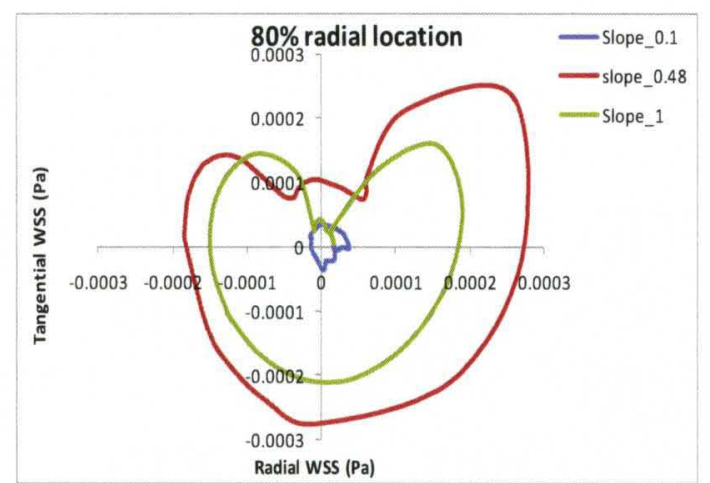

Figure 3.7. Tangential vs. radial WSS plots below, at, and above the transition at four radial locations for Set 1. Figure 3.7A, B, C, and D show plots at $20 \%, 40 \%, 60 \%$, and $80 \%$ radial locations, respectively.

The effects of Slope ratio on WSS were further studied in Set 2 for constant high Stokes Number of 10.0 and low Froude Number of 0.1 . The system properties are tabulated in Table 3.3A. Ten initial cases were run to find the transition and from free surface analysis, the transition was found to be between a Slope ratio of 2 and 3. Four more transition cases were then run (Table 3.3B) between these values and the transition was determined to be at a Slope ratio of 2.4. 
Table 3.3A. Initial cases for Set 2 and related system properties

\begin{tabular}{|c|c|c|c|c|c|c|c|c|c|c|}
\hline Cases & Gravity & $\begin{array}{c}\text { Radius } \\
\text { of } \\
\text { orbit } \\
\text { cm }\end{array}$ & $\begin{array}{l}\text { Radius } \\
\text { of dish }\end{array}$ & $\begin{array}{l}\text { Mean } \\
\text { height } \\
\text { of } \\
\text { fluid } \\
\mathrm{cm}\end{array}$ & $\begin{array}{c}\text { Orbital } \\
\text { speed }\end{array}$ & $\begin{array}{l}\text { Kinematic } \\
\text { viscosity }\end{array}$ & $\begin{array}{c}\text { Slope } \\
\text { ratio }\end{array}$ & $\begin{array}{c}\text { Froude } \\
\text { No. }\end{array}$ & $\begin{array}{c}\text { Stokes } \\
\text { No. }\end{array}$ & $\begin{array}{c}\text { Reynolds } \\
\text { No. }\end{array}$ \\
\hline 30 & 9.8 & 1.2 & 1.2 & 1.2 & 9.0370 & $1.3013 \mathrm{E}-05$ & 0.1 & 0.1 & 10 & 100 \\
\hline 31 & 9.8 & 1.2 & 0.6 & 0.6 & 12.7802 & $4.6009 \mathrm{E}-06$ & 0.2 & 0.1 & 10 & 100 \\
\hline 32 & 9.8 & 1.2 & 0.4 & 0.4 & 15.6525 & $2.5044 \mathrm{E}-06$ & 0.3 & 0.1 & 10 & 100 \\
\hline 33 & 9.8 & 1.2 & 0.3 & 0.3 & 18.0739 & $1.6267 \mathrm{E}-06$ & 0.4 & 0.1 & 10 & 100 \\
\hline 34 & 9.8 & 1.2 & 0.24 & 0.24 & 20.2073 & $1.1639 \mathrm{E}-06$ & 0.5 & 0.1 & 10 & 100 \\
\hline 35 & 9.8 & 1.2 & 0.2 & 0.2 & 22.1359 & $8.8544 \mathrm{E}-07$ & 0.6 & 0.1 & 10 & 100 \\
\hline 36 & 9.8 & 1.2 & 0.15 & 0.15 & 25.5604 & $5.7511 \mathrm{E}-07$ & 0.8 & 0.1 & 10 & 100 \\
\hline 37 & 9.8 & 1.2 & 0.12 & 0.12 & 28.5774 & $4.1151 \mathrm{E}-07$ & 1 & 0.1 & 10 & 100 \\
\hline 38 & 9.8 & 1.2 & 0.06 & 0.06 & 40.4145 & $1.4549 \mathrm{E}-07$ & 2 & 0.1 & 10 & 100 \\
\hline 39 & 9.8 & 1.2 & 0.04 & 0.04 & 49.4975 & $7.9196 \mathrm{E}-08$ & 3 & 0.1 & 10 & 100 \\
\hline
\end{tabular}

Table 3.3B. Transition cases for Set 2 and related system properties

\begin{tabular}{|c|c|c|c|c|c|c|c|c|c|c|}
\hline Set2 & Gravity & $\begin{array}{c}\text { Radius } \\
\text { of } \\
\text { orbit }\end{array}$ & $\begin{array}{c}\text { Radius } \\
\text { of dish }\end{array}$ & $\begin{array}{c}\text { Mean } \\
\text { height } \\
\text { of } \\
\text { fluid } \\
\mathrm{cm}\end{array}$ & $\begin{array}{c}\text { Orbital } \\
\text { speed }\end{array}$ & $\begin{array}{c}\text { Kinematic } \\
\text { viscosity }\end{array}$ & $\begin{array}{c}\text { Slope } \\
\text { ratio }\end{array}$ & $\begin{array}{c}\text { Froude } \\
\text { No. }\end{array}$ & $\begin{array}{c}\text { Stokes } \\
\text { No. }\end{array}$ & $\begin{array}{c}\text { Reynolds } \\
\text { No. }\end{array}$ \\
\hline $\mathbf{1}$ & $\mathrm{m} / \mathrm{s}^{2}$ & $\mathrm{~cm}$ & $\mathrm{~cm}$ & $\mathbf{s}$ & & & & \\
\hline $\mathbf{2}$ & $\mathbf{9 . 8}$ & $\mathbf{1 . 2}$ & $\mathbf{0 . 0 5 4}$ & $\mathbf{0 . 0 5 4 5}$ & $\mathbf{4 2 . 3 8 7 1}$ & $\mathbf{1 . 2 6 1 1 E - 0 7}$ & 2.2 & 0.1 & 10 & 100 \\
\hline $\mathbf{3}$ & $\mathbf{9 . 8}$ & $\mathbf{1 . 2}$ & $\mathbf{0 . 0 5 0}$ & $\mathbf{0 . 0 5 0}$ & $\mathbf{4 4 . 2 7 1 9}$ & $\mathbf{1 . 1 0 6 8 E - 0 7}$ & 2.4 & 0.1 & 10 & 100 \\
\hline $\mathbf{4}$ & $\mathbf{9 . 8}$ & $\mathbf{1 . 2}$ & $\mathbf{0 . 0 4 2 9}$ & $\mathbf{0 . 0 4 6 2}$ & $\mathbf{4 6 . 0 7 9 6}$ & $\mathbf{9 . 8 1 5 8 E - 0 8}$ & 2.6 & 0.1 & 10 & 100 \\
\hline $\mathbf{5}$ & $\mathbf{9 . 8}$ & $\mathbf{1 . 2}$ & $\mathbf{0 . 0 4 1 4}$ & $\mathbf{0 . 0 4 1 4}$ & $\mathbf{4 8 . 6 6 5 5}$ & $\mathbf{8 . 3 3 2 7 \mathbf { E } - \mathbf { 0 8 }}$ & 2.9 & 0.1 & 10 & 100 \\
\hline
\end{tabular}

Figure 3.8 shows the resultant WSS contours (for both a common scale and individual maximum scales) below, at, and above the Slope ratio transition for high Stokes Number of 10.0 and low Froude Number of 0.1. Below the transition at Slope ratio of 0.1 , WSS is higher in magnitude with higher regions of shear towards the center of the dish. At the transition (Slope ratio of 2.4), there is low uniform WSS throughout the dish. Above the transition at a Slope ratio of 3.0, the WSS remains low. The high Stokes number, indicating viscous flow, appears to dominate and keeps the WSS magnitude low. 
Figure 3.9A, 3.9B, 3.9C, and 3.9D illustrate the tangential vs. radial WSS plots for the three Slope ratios $(0.1,2.8$, and 3.0$)$ at $20 \%, 40 \%, 60 \%$, and $80 \%$ radial locations, respectively. The magnitude below the transition is significantly higher than at or above the transition, which indicates that a flatter surface (low slope) is necessary to generate some shear when viscous flow dominates (high Stokes). The shear is evenly distributed between tangential and radial components at low slope ratio towards the center $(20 \%$ and $40 \%)$, then the tangential component becomes predominant approaching the wall $(60 \%$ and $80 \%$ ). 
A

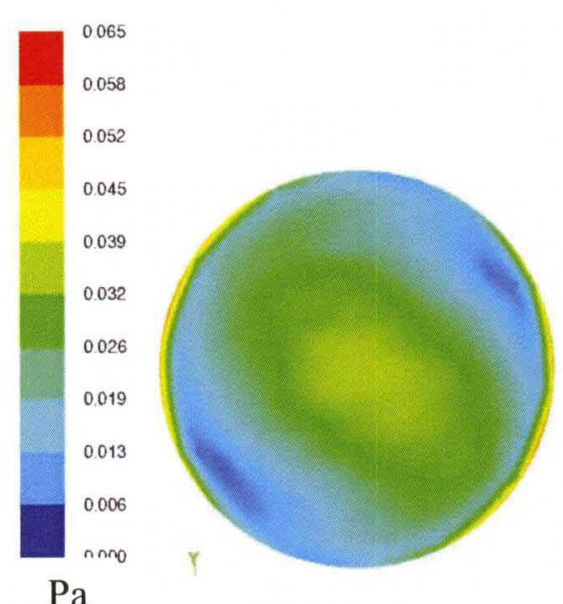

$\mathrm{Pa}$
B
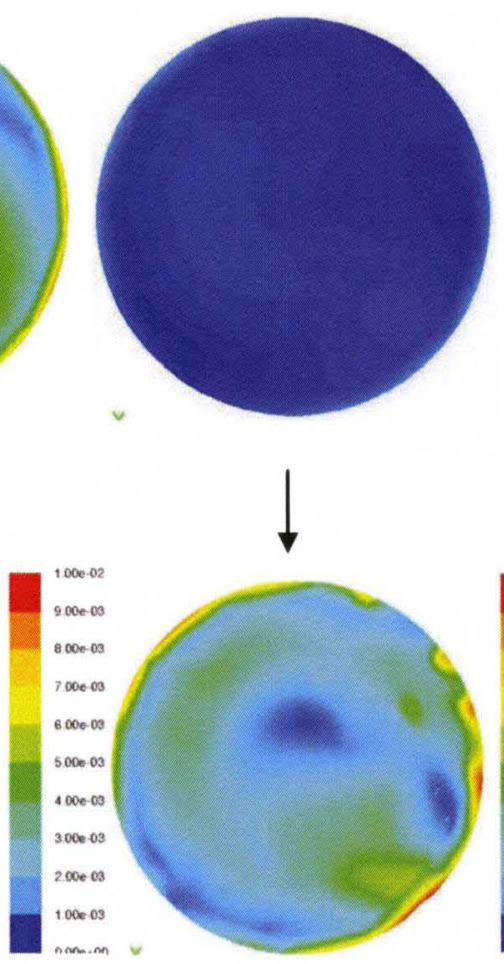

$\mathrm{Pa}$
$\mathrm{C}$
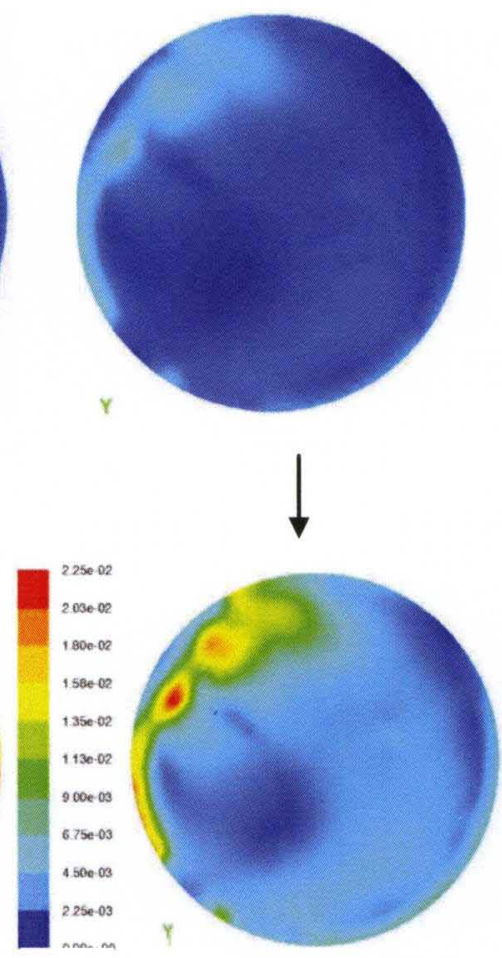

$\mathrm{Pa}$

Figure 3.8. Resultant WSS contours below, at, and above the Slope ratio transition for constant low Froude Number of 0.1 and high Stokes Number of 10. The contours are shown with a common scale in the first row and with individual maximum scales in the second row. Figure $3.8 \mathrm{~A}$ is the resultant WSS contour at a Slope ratio of 0.1 (which is well below the fluid transition); Figure 3.8B is the resultant WSS contour at a Slope ratio of 2.4 (at the transition); Figure $3.8 \mathrm{C}$ is the resultant WSS contour at a Slope ratio of 3.0 (well above the transition). 
A

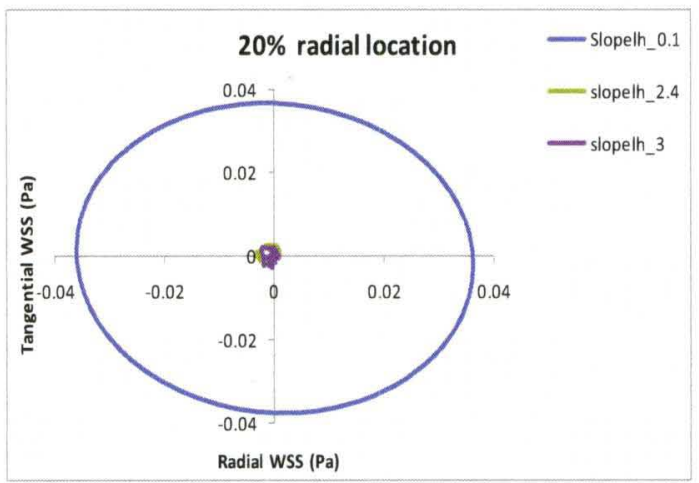

C

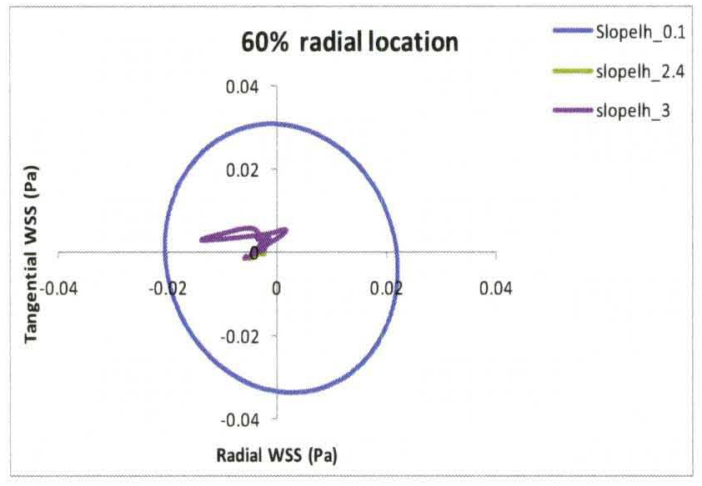

B

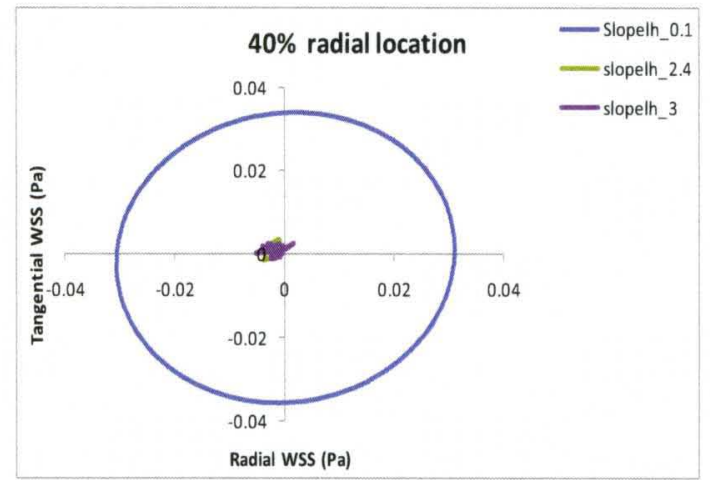

D

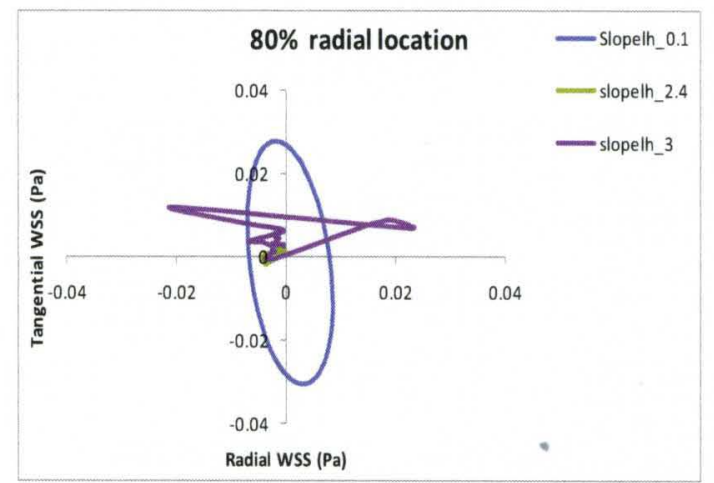

Figure 3.9. Tangential vs. radial WSS plots below, at, and above the transition at four radial locations for Set 2. Figure 3.9A, B, C, and D show plots at $20 \%, 40 \%, 60 \%$, and $80 \%$ radial locations, respectively. 


\subsubsection{WSS analysis as a function of Froude Number at constant Stokes Number and constant Slope ratio}

WSS and the Froude number transition were analyzed in Set 3 for constant low Stokes Number of 1.0 and low Slope ratio of 0.1. Froude Numbers were varied from 0.1 to 1.2 in this set. Liquid properties, dish dimensions, and orbital speed of this set are tabulated in Table 3.4A. Thirteen initial cases were run by varying the Froude Number from 0.1 to 10.0 in order to determine the fluid transition. From the free surface analysis of these initial cases, the transition point was determined to fall in between a Froude Number of 0.6 and 0.7. Five more were run between these values (Table 3.4B) and from the free surface analysis, the transition point was determined to be at a Froude Number of 0.48 .

Table 3.4A. Initial cases for Set 3 and related system properties

\begin{tabular}{|c|c|c|c|c|c|c|c|c|c|c|}
\hline Cases & Gravity & $\begin{array}{c}\text { Radius } \\
\text { of } \\
\text { orbit } \\
\mathrm{cm}\end{array}$ & $\begin{array}{l}\text { Radius } \\
\text { of dish }\end{array}$ & $\begin{array}{l}\text { Mean } \\
\text { height } \\
\text { of } \\
\text { fluid } \\
\mathrm{cm}\end{array}$ & $\begin{array}{l}\text { Orbital } \\
\text { speed }\end{array}$ & $\begin{array}{l}\text { Kinematic } \\
\text { viscosity }\end{array}$ & $\begin{array}{l}\text { Slope } \\
\text { ratio }\end{array}$ & $\begin{array}{c}\text { Froude } \\
\text { No. }\end{array}$ & $\begin{array}{l}\text { Stokes } \\
\text { No. }\end{array}$ & $\begin{array}{c}\text { Reynolds } \\
\text { No. }\end{array}$ \\
\hline 1 & $\begin{array}{l}9.8 \\
\end{array}$ & 1.2 & 1.2 & 0.012 & 0.9037 & $1.3013 \mathrm{E}-08$ & 0.1 & 0.1 & 1 & 100 \\
\hline 10 & 9.8 & 1.2 & 2.4 & 0.024 & 0.9037 & $5.2053 \mathrm{E}-08$ & 0.1 & 0.2 & 1 & 100 \\
\hline 11 & 9.8 & 1.2 & 3.6 & 0.036 & 0.9037 & $1.1712 \mathrm{E}-07$ & 0.1 & 0.3 & 1 & 100 \\
\hline 12 & 9.8 & 1.2 & 4.8 & 0.048 & 0.9037 & $2.0821 \mathrm{E}-07$ & 0.1 & 0.4 & 1 & 100 \\
\hline 13 & 9.8 & 1.2 & 6 & 0.060 & 0.9037 & $3.2533 \mathrm{E}-07$ & 0.1 & 0.5 & 1 & 100 \\
\hline 14 & 9.8 & 1.2 & 7.2 & 0.072 & 0.9037 & $4.6848 \mathrm{E}-07$ & 0.1 & 0.6 & 1 & 100 \\
\hline 15 & 9.8 & 1.2 & 12 & 0.12 & 0.9037 & $1.3013 \mathrm{E}-06$ & 0.1 & 1 & 1 & 100 \\
\hline 16 & 9.8 & 1.2 & 14.4 & 0.144 & 0.9037 & $1.8739 \mathrm{E}-06$ & 0.1 & 1.2 & 1 & 100 \\
\hline 17 & 9.8 & 1.2 & 24 & 0.24 & 0.9037 & $5.2053 \mathrm{E}-06$ & 0.1 & 2 & 1 & 100 \\
\hline 18 & 9.8 & 1.2 & 36. & 0.36 & 0.9037 & $1.1712 \mathrm{E}-05$ & 0.1 & 3 & 1 & 100 \\
\hline 19 & 9.8 & 1.2 & 48 & 0.48 & 0.9037 & $2.0821 \mathrm{E}-05$ & 0.1 & 4 & 1 & 100 \\
\hline 20 & 9.8 & 1.2 & 60 & 0.6 & 0.9037 & $3.2533 \mathrm{E}-05$ & 0.1 & 5 & 1 & 100 \\
\hline 21 & 9.8 & 1.2 & 120 & 1.2 & 0.9037 & $1.3013 \mathrm{E}-04$ & 0.1 & 10 & 1 & 100 \\
\hline
\end{tabular}


Table 3.4B. Transition cases for Set 3 and related system properties

\begin{tabular}{|c|c|c|c|c|c|c|c|c|c|c|}
\hline Set3 & Gravity & $\begin{array}{c}\text { Radius } \\
\text { of } \\
\text { orbit }\end{array}$ & $\begin{array}{c}\text { Radius } \\
\text { of dish }\end{array}$ & $\begin{array}{c}\text { Mean } \\
\text { height } \\
\text { of } \\
\text { fluid } \\
\mathrm{cm}\end{array}$ & $\begin{array}{c}\text { Orbital } \\
\text { speed }\end{array}$ & $\begin{array}{c}\text { Kinematic } \\
\text { viscosity }\end{array}$ & $\begin{array}{c}\text { Slope } \\
\text { ratio }\end{array}$ & $\begin{array}{c}\text { Froude } \\
\text { No. }\end{array}$ & $\begin{array}{c}\text { Stokes } \\
\text { No. }\end{array}$ & $\begin{array}{c}\text { Reynolds } \\
\text { No. }\end{array}$ \\
Cases & $\mathrm{m} / \mathrm{s}^{2}$ & $\mathrm{~cm}$ & $\mathrm{~cm}$ & & & & \\
\hline $\mathbf{1}$ & $\mathbf{9 . 8}$ & $\mathbf{1 . 2}$ & $\mathbf{7 . 6 8}$ & $\mathbf{0 . 0 7 6 8}$ & $\mathbf{0 . 9 0 3 7}$ & $\mathbf{5 . 3 3 0 2 E - 0 7}$ & 0.1 & 0.64 & 1 & 100 \\
\hline $\mathbf{2}$ & $\mathbf{9 . 8}$ & $\mathbf{1 . 2}$ & $\mathbf{8 . 1 6}$ & $\mathbf{0 . 0 8 1 6}$ & $\mathbf{0 . 9 0 3 7}$ & $\mathbf{6 . 0 1 7 3 E - 0 7}$ & 0.1 & 0.68 & 1 & 100 \\
\hline $\mathbf{3}$ & $\mathbf{9 . 8}$ & $\mathbf{1 . 2}$ & $\mathbf{8 . 6 4}$ & $\mathbf{0 . 0 8 6 4}$ & $\mathbf{0 . 9 0 3 7}$ & $\mathbf{6 . 7 4 6 1 E - 0 7}$ & 0.1 & 0.72 & 1 & 100 \\
\hline $\mathbf{4}$ & $\mathbf{9 . 8}$ & $\mathbf{1 . 2}$ & $\mathbf{9 . 1 2}$ & $\mathbf{0 . 0 9 1 2}$ & $\mathbf{0 . 9 0 3 7}$ & $\mathbf{7 . 5 1 6 4 E - 0 7}$ & 0.1 & 0.76 & 1 & 100 \\
\hline $\mathbf{5}$ & $\mathbf{9 . 8}$ & $\mathbf{1 . 2}$ & $\mathbf{9 . 3 6}$ & $\mathbf{0 . 0 9 3 6}$ & $\mathbf{0 . 9 0 3 7}$ & $\mathbf{7 . 9 1 7 2 E - 0 7}$ & 0.1 & 0.78 & 1 & 100 \\
\hline
\end{tabular}

Figure 3.10 shows the resultant WSS contours below, at, and above the Froude transition. The analysis demonstrates that WSS is very low and uniform below the transition at Froude Number of 0.1. There WSS is higher at the transition (Froude number $=0.48)$ ), with the maximum shear region appearing near the center. Further, above the transition at a Froude Number of 1.2, the WSS is even higher and the maximum also appears near the center. Interestingly, the shear distribution patterns are similar at and above the transition. The increasing shear with increasing Froude number is due to the higher convective forces imparting shear on the surface.

Plots of radial and tangential WSS components for varying Froude Number at four radial locations $(20 \%, 40 \%, 60 \%$, and $80 \%)$ appear in Figure 3.11 . Results demonstrate that WSS is fairly evenly distributed between radial and tangential components at $20 \%, 40 \%$ and $60 \%$ radial locations and are similar in magnitude regardless of proximity to the transition. The distribution becomes dominant in tangential direction near the wall ( $80 \%$ radial location), which is due to the steepness of the leading edge of the wave near the wall at high Froude Number. 
A

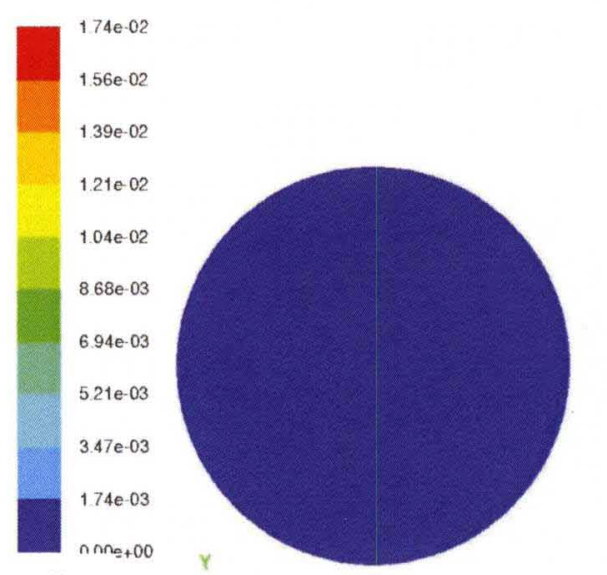

$\mathrm{Pa}$
B

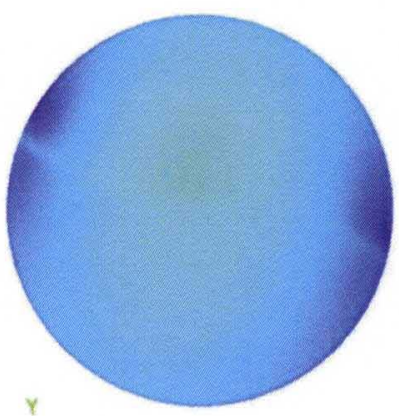

צ

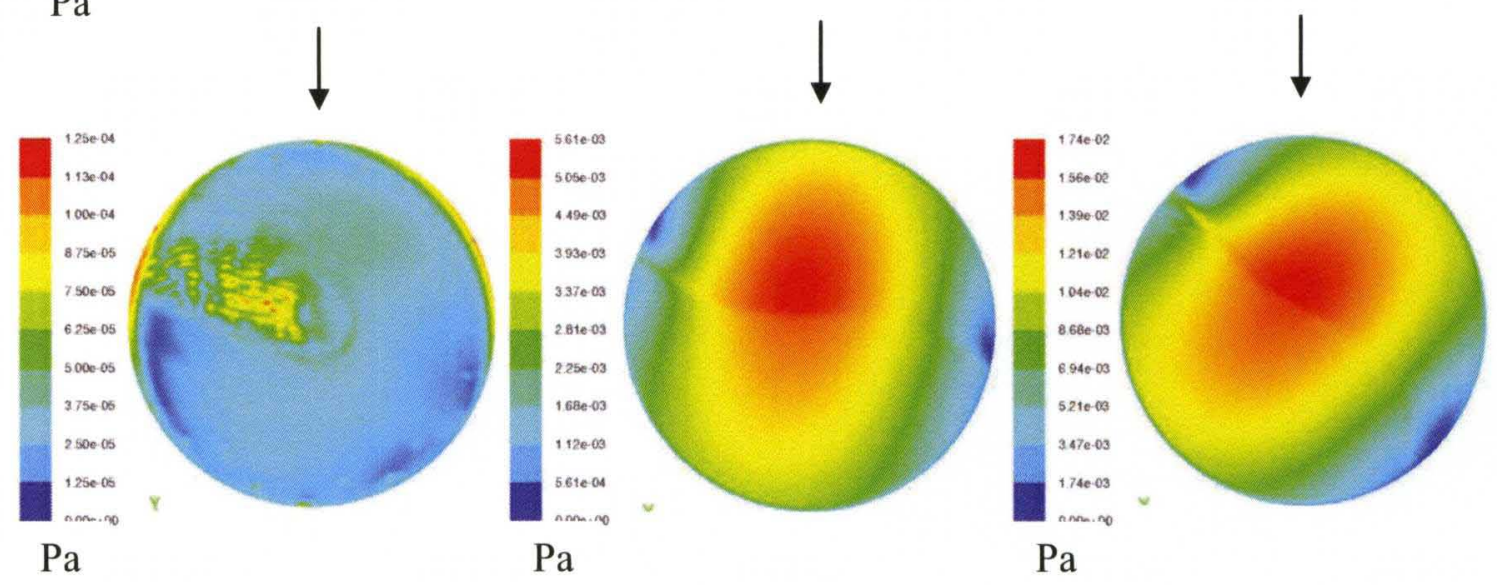

Figure 3.10. Resultant WSS contours below, at, and above the Froude transition for constant low Slope ratio of 0.1 and low Stokes Number of 1.0. The contours are shown with a common scale in the first row and with individual maximum scales in the second row. Figure $3.10 \mathrm{~A}$ is the resultant WSS contour at a Froude Number of 0.1 (which is well below the fluid transition); Figure 3.10B is the resultant WSS contour at a Froude Number of 0.64 (at the transition); Figure 3.10C is the resultant WSS contour at a Froude Number of 1.2 (well above the transition). 
A

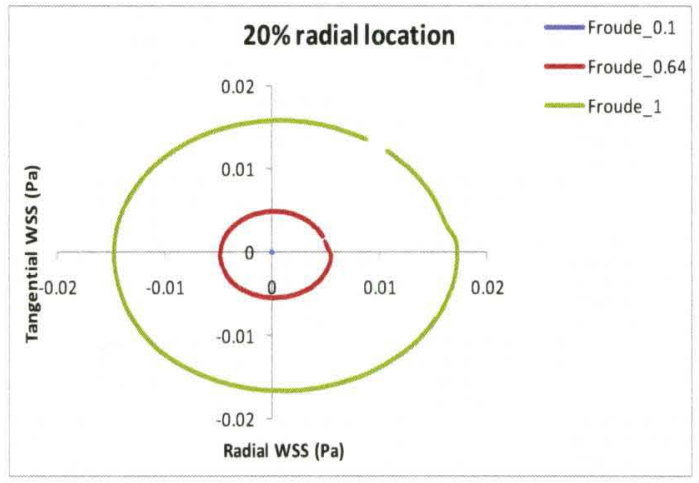

$\mathrm{C}$

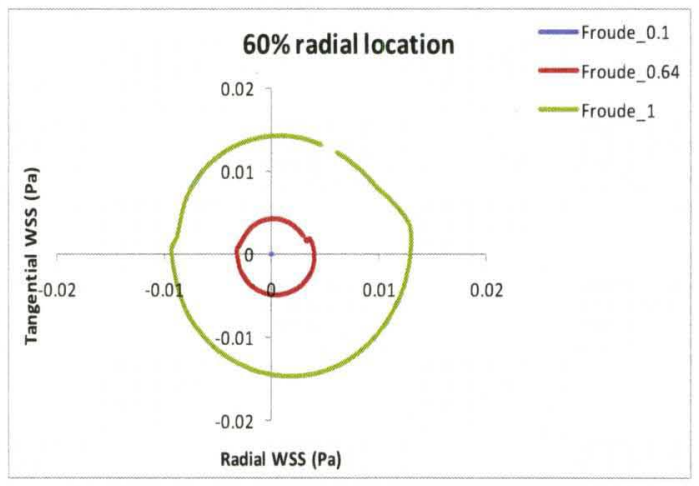

$\mathrm{B}$

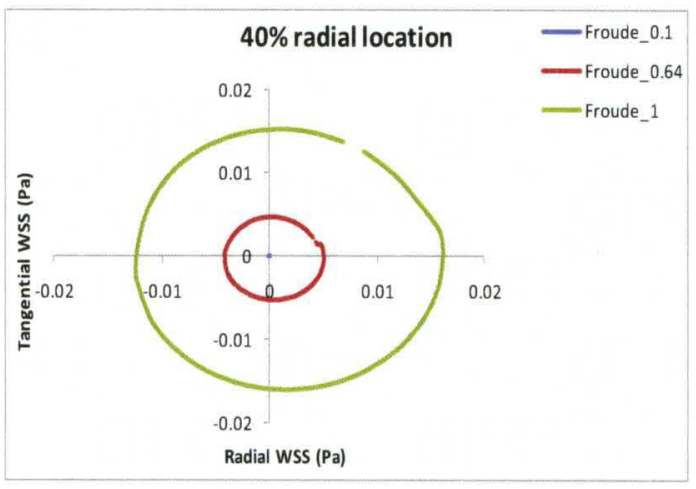

$\mathrm{D}$

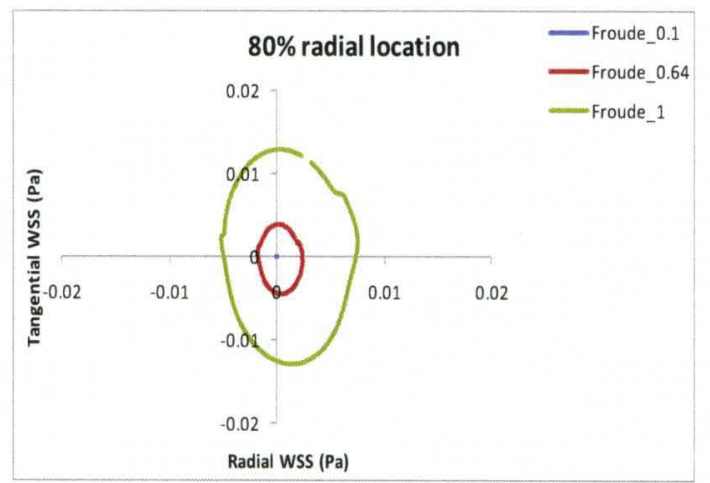

Figure 3.11. Tangential vs. radial WSS plots below, at, and above the transition at four radial locations for Set 3. Figure $3.11 \mathrm{~A}, \mathrm{~B}, \mathrm{C}$, and D show plots at $20 \%, 40 \%, 60 \%$, and $80 \%$ radial locations, respectively.

The Froude effects on WSS were next analyzed for constant high Stokes Number of 10.0 and low Slope ratio of 0.1 . The system properties are tabulated in Table 3.5A. To determine the fluid transition, 13 initial cases of varying Froude Number were run, and the transition point was thought to possibly be between a Froude Number of 2.0 and 3.0, although the Froude effects are not as apparent as seen in other cases Three more cases (Table 3.5B) were run between these values and the transition was defined at a Froude Number of 2.8, although the indications were vague. 
Table 3.5A. Initial cases for Set 4 and related system properties

\begin{tabular}{|c|c|c|c|c|c|c|c|c|c|c|}
\hline Set4 & Gravity & $\begin{array}{c}\text { Radius } \\
\text { of } \\
\text { orbit }\end{array}$ & $\begin{array}{c}\text { Radius } \\
\text { of dish }\end{array}$ & $\begin{array}{c}\text { Mean } \\
\text { height } \\
\text { of } \\
\text { fluid } \\
\text { Cases }\end{array}$ & $\begin{array}{c}\text { Orbital } \\
\text { speed }\end{array}$ & $\begin{array}{c}\text { Kinematic } \\
\text { viscosity }\end{array}$ & $\begin{array}{c}\text { Slope } \\
\text { ratio }\end{array}$ & $\begin{array}{c}\text { Froude } \\
\text { No. }\end{array}$ & $\begin{array}{c}\text { Stokes } \\
\text { No. }\end{array}$ & $\begin{array}{c}\text { Reynolds } \\
\text { No. }\end{array}$ \\
\hline $\mathbf{3 0}$ & $\mathbf{9 . 8}$ & $\mathbf{1 . 2}$ & $\mathbf{1 . 2}$ & $\mathbf{1 . 2}$ & $\mathbf{9 . 0 3 7 0}$ & $\mathbf{1 . 3 0 1 3 E - 0 5}$ & 0.1 & 0.1 & 10 & 100 \\
\hline $\mathbf{4 0}$ & $\mathbf{9 . 8}$ & $\mathbf{1 . 2}$ & $\mathbf{2 . 4}$ & $\mathbf{2 . 4}$ & $\mathbf{9 . 0 3 7 0}$ & $\mathbf{5 . 2 0 5 3 E - 0 5}$ & 0.1 & 0.2 & 10 & 100 \\
\hline $\mathbf{4 1}$ & $\mathbf{9 . 8}$ & $\mathbf{1 . 2}$ & $\mathbf{3 . 6}$ & $\mathbf{3 . 6}$ & $\mathbf{9 . 0 3 7 0}$ & $\mathbf{1 . 1 7 1 2 E - 0 4}$ & 0.1 & 0.3 & 10 & 100 \\
\hline $\mathbf{4 2}$ & $\mathbf{9 . 8}$ & $\mathbf{1 . 2}$ & $\mathbf{4 . 8}$ & $\mathbf{4 . 8}$ & $\mathbf{9 . 0 3 7 0}$ & $\mathbf{2 . 0 8 2 1 \mathbf { E } - \mathbf { 0 4 }}$ & 0.1 & 0.4 & 10 & 100 \\
\hline $\mathbf{4 3}$ & $\mathbf{9 . 8}$ & $\mathbf{1 . 2}$ & $\mathbf{6}$ & $\mathbf{6 .}$ & $\mathbf{9 . 0 3 7 0}$ & $\mathbf{3 . 2 5 3 3 \mathbf { E } - \mathbf { 0 4 }}$ & 0.1 & 0.5 & 10 & 100 \\
\hline $\mathbf{4 4}$ & $\mathbf{9 . 8}$ & $\mathbf{1 . 2}$ & $\mathbf{7 . 2}$ & $\mathbf{7 . 2}$ & $\mathbf{9 . 0 3 7 0}$ & $\mathbf{4 . 6 8 4 8 E - 0 4}$ & 0.1 & 0.6 & 10 & 100 \\
\hline $\mathbf{4 5}$ & $\mathbf{9 . 8}$ & $\mathbf{1 . 2}$ & $\mathbf{9 . 6}$ & $\mathbf{9 . 6}$ & $\mathbf{9 . 0 3 7 0}$ & $\mathbf{8 . 3 2 8 5 E - 0 4}$ & 0.1 & 0.8 & 10 & 100 \\
\hline $\mathbf{4 6}$ & $\mathbf{9 . 8}$ & $\mathbf{1 . 2}$ & $\mathbf{1 2}$ & $\mathbf{1 2}$ & $\mathbf{9 . 0 3 7 0}$ & $\mathbf{1 . 3 0 1 3 E - 0 3}$ & 0.1 & 1 & 10 & 100 \\
\hline $\mathbf{4 7}$ & $\mathbf{9 . 8}$ & $\mathbf{1 . 2}$ & $\mathbf{1 4 . 4}$ & $\mathbf{1 4 . 4}$ & $\mathbf{9 . 0 3 7 0}$ & $\mathbf{1 . 8 7 3 9 E - 0 3}$ & 0.1 & 1.2 & 10 & 100 \\
\hline $\mathbf{4 8}$ & $\mathbf{9 . 8}$ & $\mathbf{1 . 2}$ & $\mathbf{2 4}$ & $\mathbf{2 4}$ & $\mathbf{9 . 0 3 7 0}$ & $\mathbf{5 . 2 0 5 3 E - 0 3}$ & 0.1 & 2 & 10 & 100 \\
\hline 49 & $\mathbf{9 . 8}$ & $\mathbf{1 . 2}$ & $\mathbf{3 6}$ & $\mathbf{3 6}$ & $\mathbf{9 . 0 3 7 0}$ & $\mathbf{1 . 1 7 1 2 E - 0 2}$ & 0.1 & 3 & 10 & 100 \\
\hline $\mathbf{5 0}$ & $\mathbf{9 . 8}$ & $\mathbf{1 . 2}$ & $\mathbf{6 0}$ & $\mathbf{6 0}$ & $\mathbf{9 . 0 3 7 0}$ & $\mathbf{3 . 2 5 3 3 E - 0 2}$ & 0.1 & 5 & 10 & 100 \\
\hline $\mathbf{5 1}$ & $\mathbf{9 . 8}$ & $\mathbf{1 . 2}$ & $\mathbf{1 2 0}$ & $\mathbf{1 2 0}$ & $\mathbf{9 . 0 3 7 0}$ & $\mathbf{1 . 3 0 1 3 E - 0 1}$ & 0.1 & 10 & 10 & 100 \\
\hline
\end{tabular}

Table 3.5B. Transition cases for Set 4 and related system properties

\begin{tabular}{|c|c|c|c|c|c|c|c|c|c|c|}
\hline Cases & Gravity & $\begin{array}{c}\text { Radius } \\
\text { of } \\
\text { orbit } \\
\mathrm{cm} \\
\end{array}$ & $\begin{array}{l}\text { Radius } \\
\text { of dish }\end{array}$ & $\begin{array}{c}\text { Mean } \\
\text { height } \\
\text { of } \\
\text { fluid } \\
\mathrm{cm}\end{array}$ & $\begin{array}{c}\text { Orbital } \\
\text { speed }\end{array}$ & $\begin{array}{c}\text { Kinematic } \\
\text { viscosity }\end{array}$ & $\begin{array}{c}\text { Slope } \\
\text { ratio }\end{array}$ & $\begin{array}{c}\text { Froude } \\
\text { No. }\end{array}$ & $\begin{array}{c}\text { Stokes } \\
\text { No. }\end{array}$ & $\begin{array}{c}\text { Reynolds } \\
\text { No. }\end{array}$ \\
\hline 3 & 9.8 & 1.2 & 28.8 & 28.8 & 9.0370 & $7.4956 \mathrm{E}-03$ & 0.1 & 2.4 & 10 & 100 \\
\hline 4 & 9.8 & 1.2 & 31.2 & 31.2 & 9.0370 & $8.7969 \mathrm{E}-03$ & 0.1 & 2.6 & 10 & 100 \\
\hline 5 & 9.8 & 1.2 & 33.6 & 33.6 & 9.0370 & $1.0202 \mathrm{E}-02$ & 0.1 & 2.8 & 10 & 100 \\
\hline
\end{tabular}

Figure 3.12 shows the resultant WSS contours below, at, and above the possible Froude transition at low Slope ratio of 0.1 and high Stokes Number of 10.0. Very low and mostly uniform shear is observed below the transition at Froude Number of 1.0. Much higher WSS (maximum at $2.8 \mathrm{~Pa}$ ) occurs at Froude Number of 2.8 with the maximum shear occurring near the center of the dish. Interestingly, minimal shear occurs above this point at Froude Number of 5.0. The explanation of this pattern is not clear. Supercritical wave patterns might be expected, but further analysis is needed to confirm this. WSS distributions between tangential and radial components for this set appear in Figure 3.13. 
WSS magnitudes are greatest at the possible transition. WSS below this point at a Froude Number of 1.0 shows a dominant radial component near the center (at 20\%), even distribution at $40 \%$, then become elongated along the tangential axis at $60 \%$ and more so at $80 \%$ radial location. This pattern is indicative of higher tangential flow near the sidewall and indicates that the non-oscillating flow associated with high DOSI values is mostly in the tangential direction. 
A
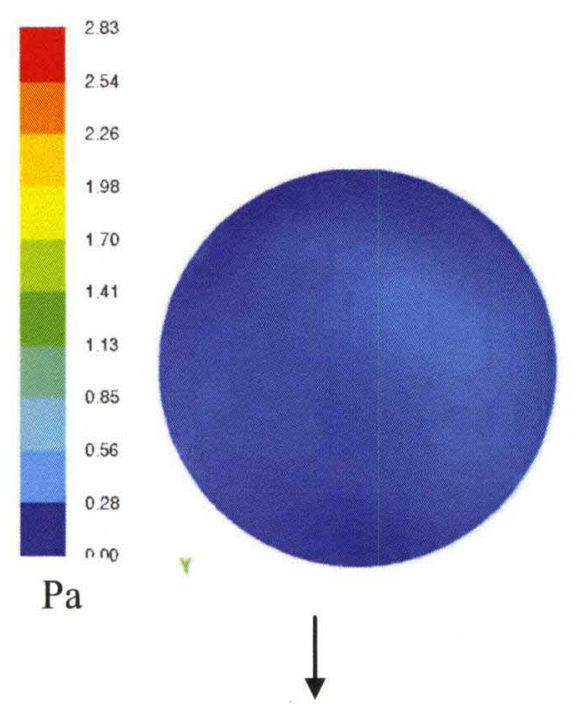

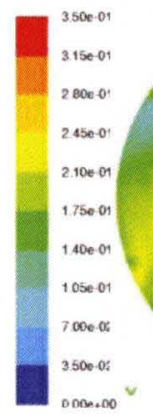

$\mathrm{Pa}$
B
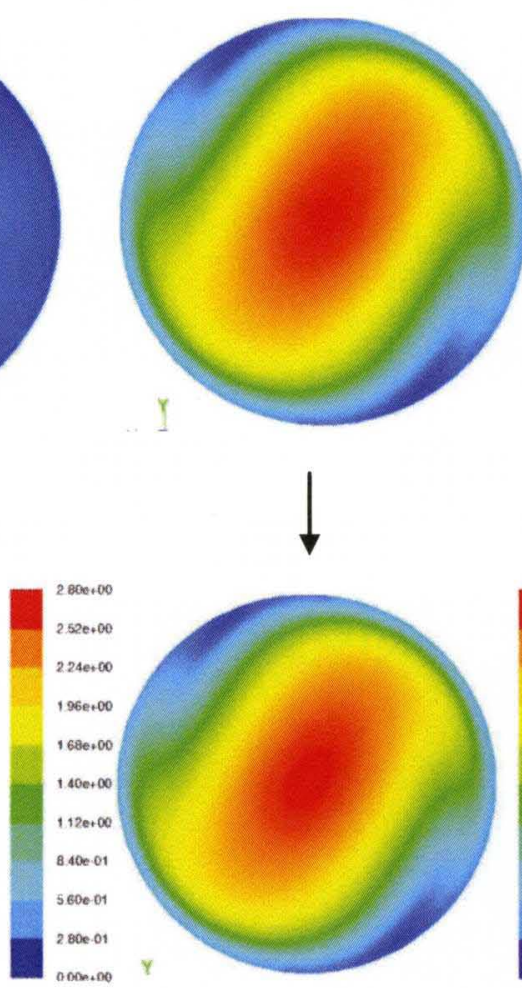

$\mathrm{Pa}$
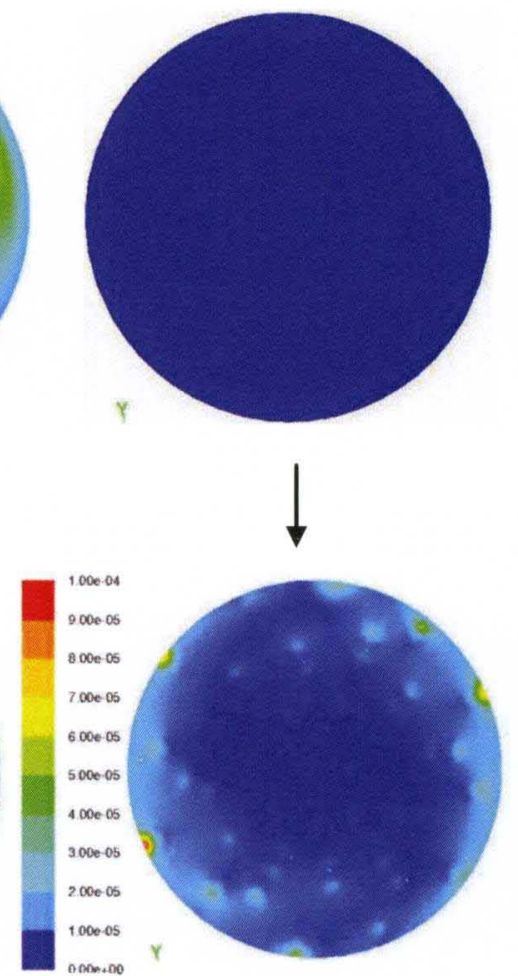

$\mathrm{Pa}$

Figure 3.12. Resultant WSS contours below, at, and above the Froude transition for constant low Slope ratio of 0.1 and high Stokes Number of 10.0. The contours are shown with a common scale in the first row and with individual maximum scales in the second row. Figure 3.12A is the resultant WSS contour at Froude Number of 1.0 (which is well below the fluid transition); Figure 3.12B is the resultant WSS contour at a Froude Number of 2.8 (at the transition); Figure $3.12 \mathrm{C}$ is the resultant WSS contour at a Froude Number of 5.0 (well above the transition). 
A

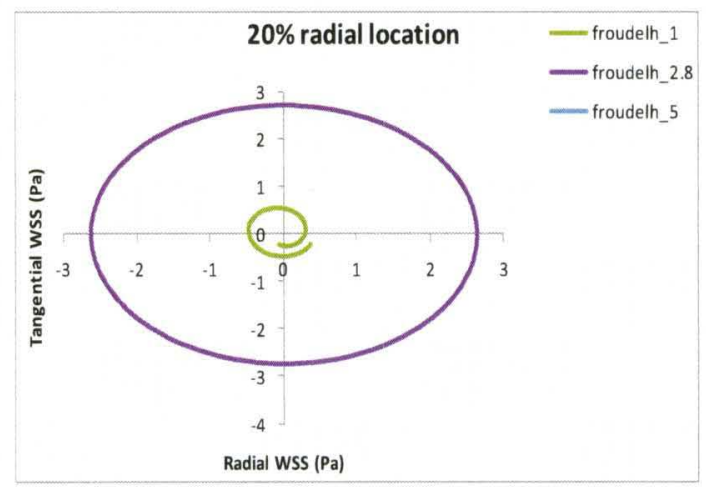

$\mathrm{C}$

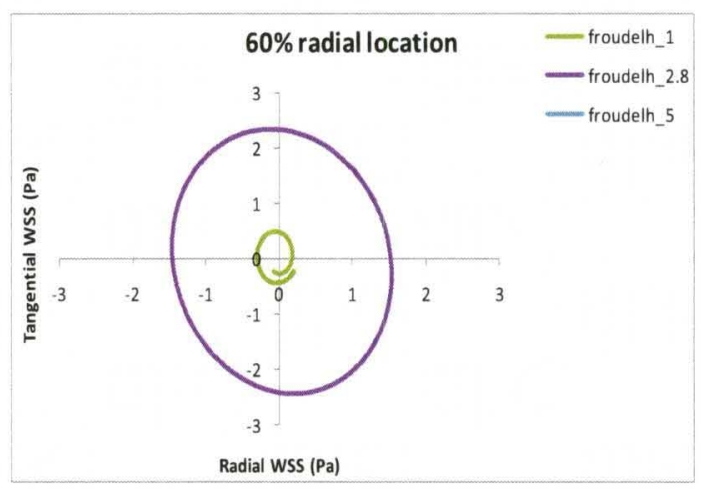

B

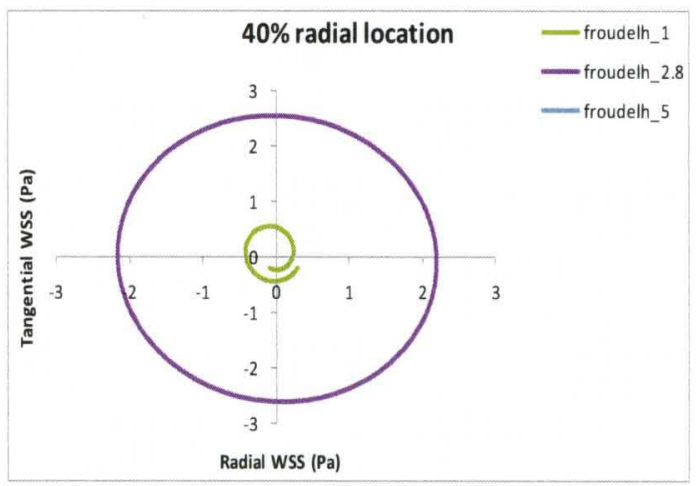

$\mathrm{D}$

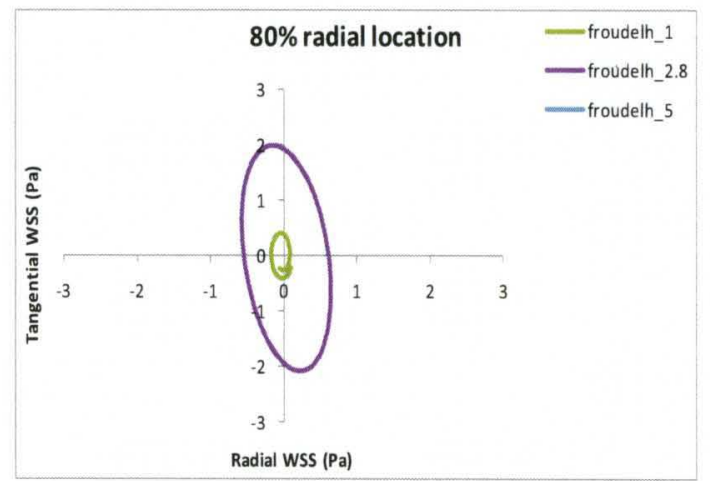

Figure 3.13. Tangential vs. radial WSS plots below, at, and above the transition at four radial locations for Set 4 . Figure $3.13 \mathrm{~A}, \mathrm{~B}, \mathrm{C}$, and D show plots at $20 \%, 40 \%, 60 \%$, and $80 \%$ radial locations, respectively. 


\subsubsection{WSS analysis as a function of Stokes Number at constant Froude Number and}

constant Slope ratio

WSS and the Stokes number transition were analyzed in Set 5 for constant low Froude Number of 0.1 and low Slope ratio of 0.1 . Stokes Numbers were initially varied from 1 to 10 . Liquid properties, dish dimensions, and orbital speed of this set are tabulated in Table 3.6A. From the free surface analysis of these initial cases, the transition point was determined to occur in between a Stokes Number of 5 and 6 . Five more cases were run between these values (Table 3.6B) and from the free surface analysis, the transition point was determined to occur at a Stokes Number of 5.2.

Table 3.6A. Initial cases for Set 5 and related system properties

\begin{tabular}{|c|c|c|c|c|c|c|c|c|c|c|}
\hline Set5 & Gravity & $\begin{array}{c}\text { Radius } \\
\text { of } \\
\text { orbit } \\
\text { cm }\end{array}$ & $\begin{array}{l}\text { Radius } \\
\text { of dish }\end{array}$ & $\begin{array}{l}\text { Mean } \\
\text { height } \\
\text { of } \\
\text { fluid } \\
\mathrm{Cm}\end{array}$ & $\begin{array}{c}\text { Orbital } \\
\text { speed }\end{array}$ & $\begin{array}{l}\text { Kinematic } \\
\text { viscosity }\end{array}$ & $\begin{array}{l}\text { Slope } \\
\text { ratio }\end{array}$ & $\begin{array}{c}\text { Froude } \\
\text { No. }\end{array}$ & $\begin{array}{c}\text { Stokes } \\
\text { No. }\end{array}$ & $\begin{array}{c}\text { Reynolds } \\
\text { No. }\end{array}$ \\
\hline 1 & 9.8 & 1.2 & 1.2 & 0.0120 & 0.9037 & $1.3013 \mathrm{E}-08$ & 0.1 & 0.1 & 1 & 100 \\
\hline 22 & 9.8 & 1.2 & 1.2 & 0.0480 & 1.8074 & 1.0411E-07 & 0.1 & 0.1 & 2 & 100 \\
\hline 23 & 9.8 & 1.2 & 1.2 & 0.1080 & 2.7111 & 3.5136E-07 & 0.1 & 0.1 & 3 & 100 \\
\hline 24 & 9.8 & 1.2 & 1.2 & 0.1920 & 3.6148 & 8.3285E-07 & 0.1 & 0.1 & 4 & 100 \\
\hline 25 & 9.8 & 1.2 & 1.2 & 0.3000 & 4.5185 & $1.6267 \mathrm{E}-06$ & 0.1 & 0.1 & 5 & 100 \\
\hline 26 & 9.8 & 1.2 & 1.2 & 0.4320 & 5.4222 & $2.8109 \mathrm{E}-06$ & 0.1 & 0.1 & 6 & 100 \\
\hline 27 & 9.8 & 1.2 & 1.2 & 0.5880 & 6.3259 & $4.4635 \mathrm{E}-06$ & 0.1 & 0.1 & 7 & 100 \\
\hline 28 & 9.8 & 1.2 & 1.2 & 0.7680 & 7.2296 & $6.6628 \mathrm{E}-06$ & 0.1 & 0.1 & 8 & 100 \\
\hline 29 & 9.8 & 1.2 & 1.2 & 1.2000 & 9.0370 & $1.3013 \mathrm{E}-05$ & 0.1 & 0.1 & 10 & 100 \\
\hline
\end{tabular}

Table 3.6B. Transition cases for Set 5 and system properties

\begin{tabular}{|c|c|c|c|c|c|c|c|c|c|c|}
\hline Set5 & Gravity & $\begin{array}{c}\text { Radius } \\
\text { of } \\
\text { orbit }\end{array}$ & $\begin{array}{c}\text { Radius } \\
\text { of dish }\end{array}$ & $\begin{array}{c}\text { Mean } \\
\text { height } \\
\text { of } \\
\text { fluid } \\
\text { Cm }\end{array}$ & $\begin{array}{c}\text { Orbital } \\
\text { speed }\end{array}$ & $\begin{array}{c}\text { Kinematic } \\
\text { viscosity }\end{array}$ & $\begin{array}{c}\text { Slope } \\
\text { ratio }\end{array}$ & $\begin{array}{c}\text { Froude } \\
\text { No. }\end{array}$ & $\begin{array}{c}\text { Stokes } \\
\text { No. }\end{array}$ & $\begin{array}{c}\text { Reynolds } \\
\text { No. }\end{array}$ \\
\hline $\mathbf{1}$ & $\mathrm{m} / \mathrm{s}^{2}$ & $\mathrm{~cm}$ & $\mathrm{~cm}$ & $\mathbf{s}$ & & & & \\
\hline $\mathbf{2}$ & $\mathbf{9 . 8}$ & $\mathbf{1 . 2}$ & $\mathbf{1 . 2}$ & $\mathbf{0 . 3 2 4 5}$ & $\mathbf{4 . 6 9 9 2}$ & $\mathbf{1 . 8 2 9 8 E - 0 6}$ & 0.1 & 0.1 & 5.2 & 100 \\
\hline $\mathbf{3}$ & $\mathbf{9 . 8}$ & $\mathbf{1 . 2}$ & $\mathbf{1 . 2}$ & $\mathbf{0 . 3 4 9 9}$ & $\mathbf{4 . 8 8 0 0}$ & $\mathbf{2 . 0 4 9 1 E - 0 6}$ & 0.1 & 0.1 & 5.4 & 100 \\
\hline $\mathbf{4}$ & $\mathbf{9 . 8}$ & $\mathbf{1 . 2}$ & $\mathbf{1 . 2}$ & $\mathbf{0 . 3 7 6 3}$ & $\mathbf{5 . 0 6 0 7}$ & $\mathbf{2 . 2 8 5 3 E - 0 6}$ & 0.1 & 0.1 & 5.6 & 100 \\
\hline $\mathbf{5}$ & $\mathbf{9 . 8}$ & $\mathbf{1 . 2}$ & $\mathbf{1 . 2}$ & $\mathbf{0 . 4 1 7 7}$ & $\mathbf{5 . 3 4 1 4}$ & $\mathbf{2 . 5 3 9 0 E - 0 6}$ & 0.1 & 0.1 & 5.8 & 100 \\
\hline
\end{tabular}


Figure 3.14 shows the resultant WSS contours below, at, and above the Stokes transition at low Slope ratio of 0.1 and low Froude Number of 0.1 . The shear contour below the transition at a Stokes Number of 1.0 indicates minimal shear. The contour for the Stokes transition value of 5.2 still indicates very low shear but indicates a maximum region of shear near the center of the dish. Higher WSS values are observed well above the transition (Stokes Number of 10.0). The maximum shear region appears as a tiny layer near the side wall, and a much larger region but of somewhat lower magnitude appears near the center.

Plots of radial and tangential WSS components as a function of Stokes Number at four radial locations $(20 \%, 40 \%, 60 \%$, and $80 \%)$ appear in Figure 3.15 . WSS is negligible below the transition (Stokes Number of 1.0), whereas higher shear ranges are observed above the transition (Stokes Number of 10.0), although the magnitudes are still very low relative to arterial values. The low magnitudes are the result of viscous dominated flow at high Stokes number. The shear is evenly distributed near the center ( $20 \%$ and $40 \%$ radial locations), and becomes increasingly dominated by its tangential component as radius increases. 
A

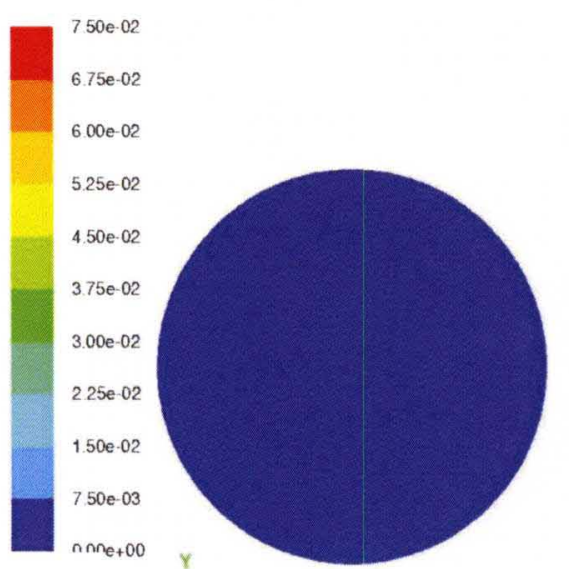

$\mathrm{Pa}$
B
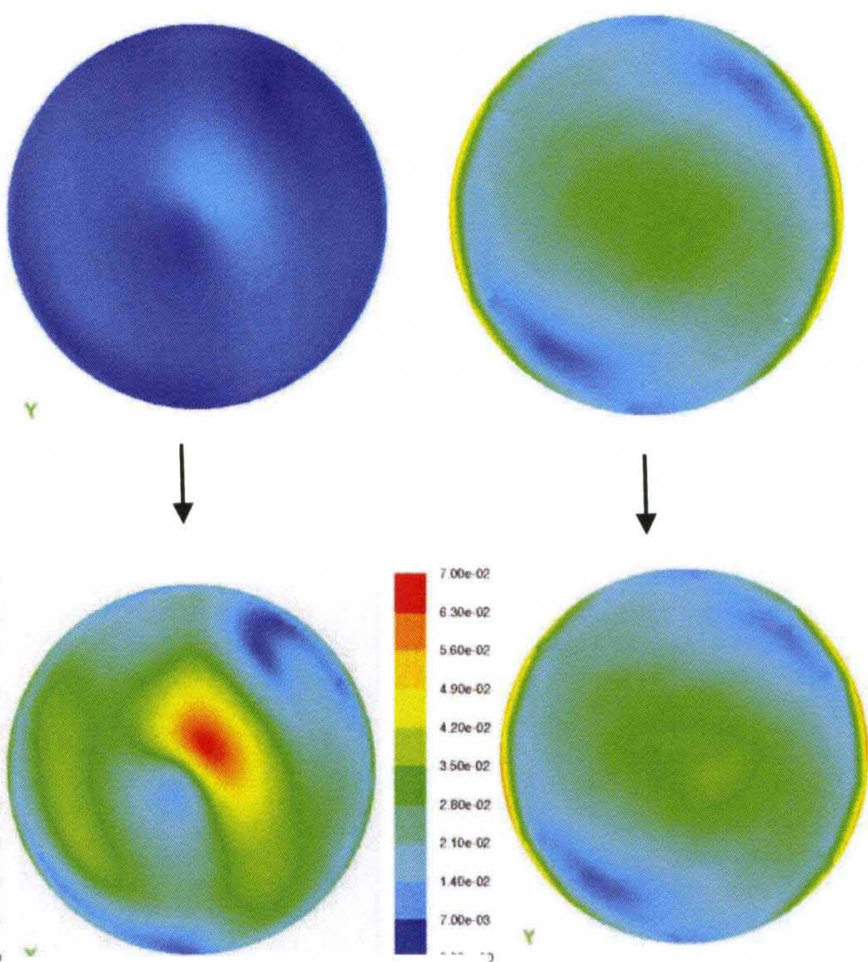

$\mathrm{Pa}$

Figure 3.14. Resultant WSS contours below, at, and above the Stokes transition for constant low Slope ratio of 0.1 and low Froude Number of 0.1 . The contours are shown with a common scale in the first row and with individual maximum scales in the second row. Figure 3.14A is the resultant WSS contour at Stokes Number of 1.0 (which is well below the fluid transition); Figure 3.14B is the resultant WSS contour at a Stokes Number of 5.2 (at the transition); Figure 3.14C is the resultant WSS contour at a Stokes Number of 10.0 (well above the transition). 
A

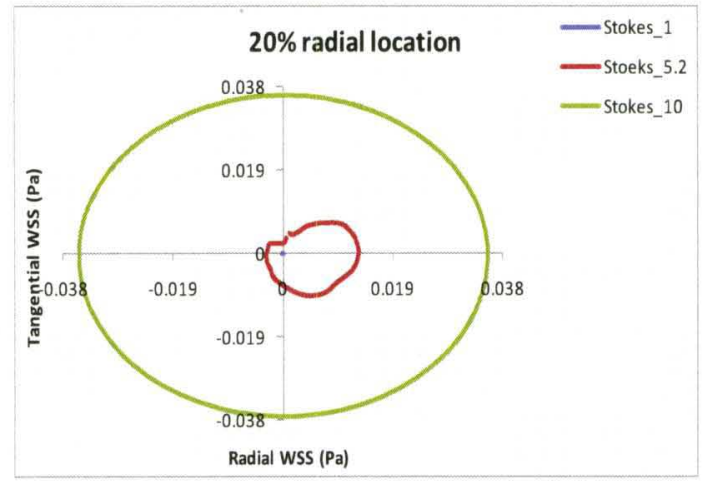

$\mathrm{C}$

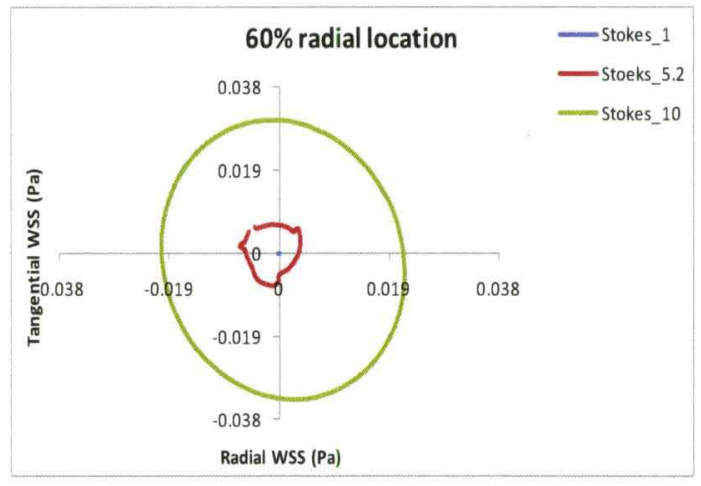

B

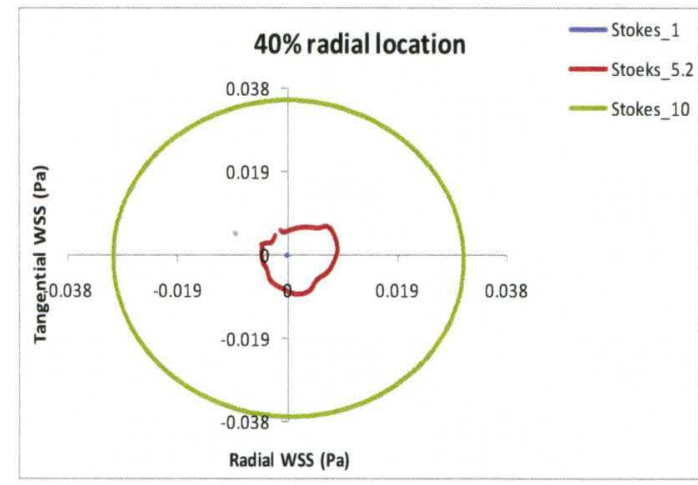

$\mathrm{D}$

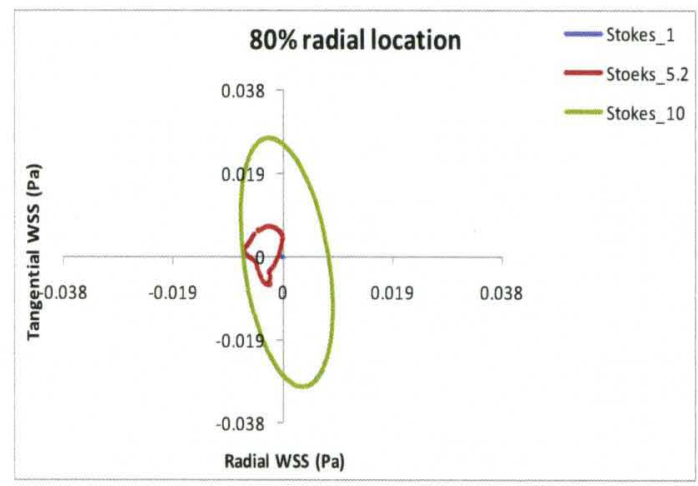

Figure 3.15. Tangential vs. radial WSS plots below, at, and above the transition at four radial locations for Set 5. Figure 3.15A, B, C, and D show plots at $20 \%, 40 \%, 60 \%$, and $80 \%$ radial locations, respectively.

Stokes effects on WSS were analyzed in Set 6 for constant high Froude Number of 1.2 and low Slope ratio of 0.1. Initial cases were run with Stokes Number varying from 1 to 10 . The system properties are tabulated in Table 3.7A. The transition point was determined to be between a Stokes Number of 3.0 and 4.0. The transition point was determined to occur at a Stokes Number of 3.2 after analyzing 5 more cases between a Stokes Number of 3.0 and 4.0 (Table3.7B). 
Table 3.7A. Initial cases for Set 6 and related system properties

\begin{tabular}{|c|c|c|c|c|c|c|c|c|c|c|}
\hline Cases & Gravity & $\begin{array}{c}\text { Radius } \\
\text { of orbit } \\
\text { cm }\end{array}$ & $\begin{array}{c}\text { Radius } \\
\text { of dish } \\
\\
\mathrm{cm}\end{array}$ & $\begin{array}{c}\text { Mean } \\
\text { height } \\
\text { of fluid } \\
\mathrm{cm}\end{array}$ & $\begin{array}{c}\text { Orbital } \\
\text { speed }\end{array}$ & $\begin{array}{c}\text { Kinematic } \\
\text { viscosity } \\
\mathrm{m}^{2} / \mathrm{s}\end{array}$ & $\begin{array}{c}\text { Slope } \\
\text { ratio }\end{array}$ & $\begin{array}{c}\text { Froude } \\
\text { No. }\end{array}$ & $\begin{array}{c}\text { Stokes } \\
\text { No. }\end{array}$ & $\begin{array}{c}\text { Reynolds } \\
\text { No. }\end{array}$ \\
\hline 52 & 9.8 & 1.2 & 14.4 & 0.144 & 0.9037 & $1.8739 \mathrm{E}-06$ & 0.1 & 1.2 & 1 & 100 \\
\hline 53 & 9.8 & 1.2 & 14.4 & 0.576 & 1.8074 & $1.4991 \mathrm{E}-05$ & 0.1 & 1.2 & 2 & 100 \\
\hline 54 & 9.8 & 1.2 & 14.4 & 1.296 & 2.7111 & 5.0595E-05 & 0.1 & 1.2 & 3 & 100 \\
\hline 55 & 9.8 & 1.2 & 14.4 & 2.304 & 3.6148 & 1.1993E-04 & 0.1 & 1.2 & 4 & 100 \\
\hline 56 & 9.8 & 1.2 & 14.4 & 3.6 & 4.5185 & $2.3424 \mathrm{E}-04$ & 0.1 & 1.2 & 5 & 100 \\
\hline 57 & 9.8 & 1.2 & 14.4 & 5.184 & 5.4222 & $4.0476 \mathrm{E}-04$ & 0.1 & 1.2 & 6 & 100 \\
\hline 58 & 9.8 & 1.2 & 14.4 & 7.056 & 6.3259 & 6.4275E-04 & 0.1 & 1.2 & 7 & 100 \\
\hline 59 & 9.8 & 1.2 & 14.4 & 9.216 & 7.2296 & $9.5944 \mathrm{E}-04$ & 0.1 & 1.2 & 8 & 100 \\
\hline 60 & 9.8 & 1.2 & 14.4 & 14.4 & 9.0370 & $1.8739 \mathrm{E}-03$ & 0.1 & 1.2 & 10 & 100 \\
\hline
\end{tabular}

Table 3.7B. Transition cases for Set 6 and system properties

\begin{tabular}{|c|c|c|c|c|c|c|c|c|c|c|}
\hline Cases & Gravity & $\begin{array}{c}\text { Radius } \\
\text { of } \\
\text { orbit } \\
\mathrm{cm}\end{array}$ & $\begin{array}{l}\text { Radius } \\
\text { of dish }\end{array}$ & $\begin{array}{l}\text { Mean } \\
\text { height } \\
\text { of } \\
\text { fluid } \\
\mathrm{cm}\end{array}$ & $\begin{array}{c}\text { Orbital } \\
\text { speed }\end{array}$ & $\begin{array}{c}\text { Kinematic } \\
\text { viscosity } \\
\mathrm{m}^{2} / \mathrm{s}\end{array}$ & $\begin{array}{l}\text { Slope } \\
\text { ratio }\end{array}$ & $\begin{array}{c}\text { Froude } \\
\text { No. }\end{array}$ & $\begin{array}{l}\text { Stokes } \\
\text { No. }\end{array}$ & $\begin{array}{c}\text { Reynolds } \\
\text { No. }\end{array}$ \\
\hline 1 & 9.8 & 1.2 & 14.4 & 1.4746 & 2.8918 & $6.1404 \mathrm{E}-05$ & 0.1 & 1.2 & 3.2 & 100 \\
\hline 2 & 9.8 & 1.2 & 14.4 & 1.6646 & 3.0726 & $7.3652 \mathrm{E}-05$ & 0.1 & 1.2 & 3.4 & 100 \\
\hline 3 & 9.8 & 1.2 & 14.4 & 1.8662 & 3.2533 & & 0.1 & 1.2 & 3.6 & 100 \\
\hline 4 & 9.8 & 1.2 & 14.4 & 2.0794 & 3.4340 & $1.0282 \mathrm{E}-04$ & 0.1 & 1.2 & 3.8 & 100 \\
\hline 5 & 9.8 & 1.2 & 14.4 & 2.192 & 3.5244 & $1.1116 \mathrm{E}-04$ & 0.1 & 1.2 & 3.9 & 100 \\
\hline
\end{tabular}

Figure 3.16 shows the resultant WSS contours below, at, and above the Stokes transition at low Slope ratio of 0.1 and high Froude Number of 1.2. With the common scale, the shear contours have negligible shear below and at the transition. Shear is significantly higher above the transition, and the region of maximum shear again occurs near the center and decreases towards the sidewall.

Plots of radial and tangential WSS components as a function of Stokes Number at four radial locations $(20 \%, 40 \%, 60 \%$, and $80 \%)$ appear in Figure 3.17. The WSS distribution trends are virtually the same as the previous set, again due to the viscous dominated flow for high Stokes numbers. Distribution between tangential and radial components is even closer to the center and becomes tangential dominated moving outward towards the sidewall. However, the magnitude is two orders of magnitude 
higher than the previous set, the only difference being that the Froude number is above the transition in this set whereas it was below the transition in the previous set. The Stokes number and Slope ratio for Figure 3.16C are the same as Figure 3.12C and both are at Froude numbers above the transition. They differ in that the Froude number is 1.2 for Figure 3.16C and is 5 for Figure 3.12C. It is possible that there is increased turbulence as Froude number increases, which results in the lower shear seen for the higher Froude case. 
A

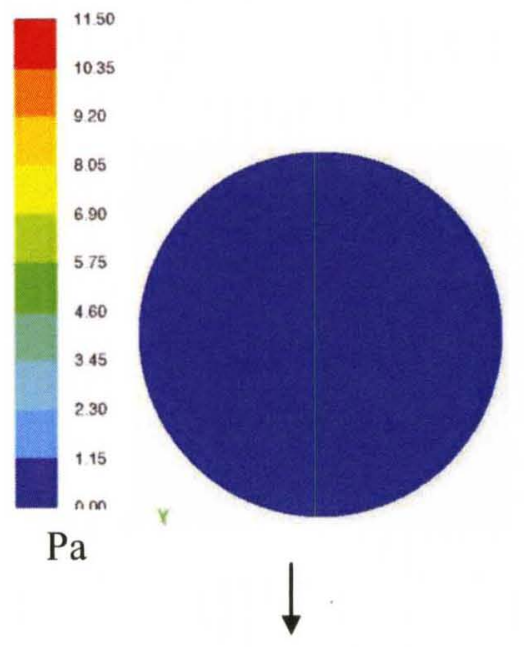

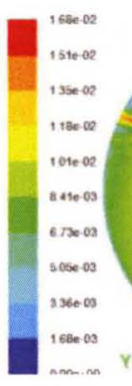

$\mathrm{Pa}$
B
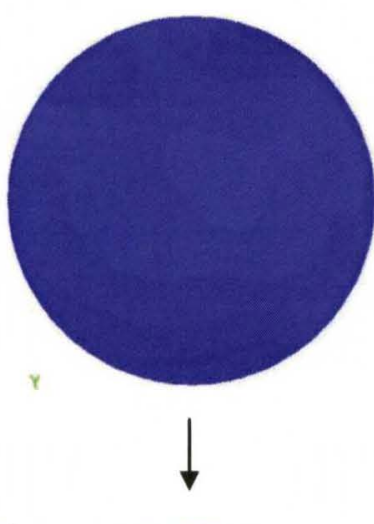

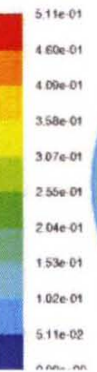

$\mathrm{Pa}$

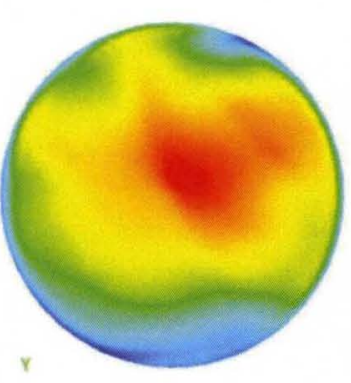

$\mathrm{C}$
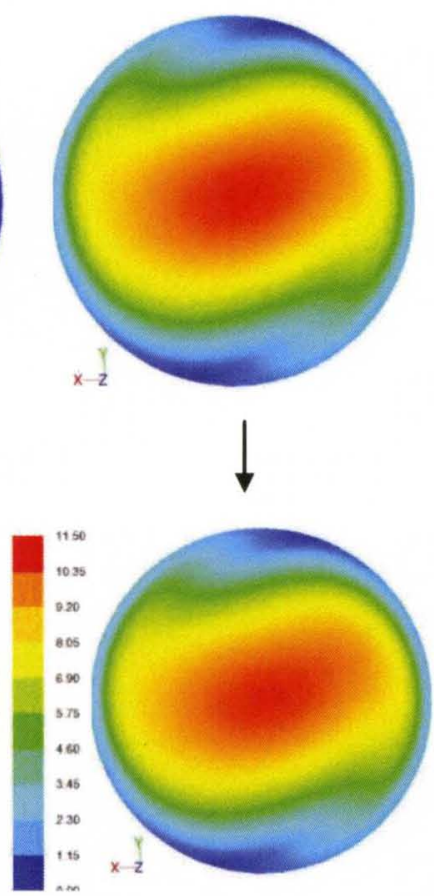

$\mathrm{Pa}$

Figure 3.16. Resultant WSS contours below, at, and above the Stokes transition for constant low Slope ratio of 0.1 and high Froude Number of 1.2. The contours are shown with a common scale in the first row and with individual maximum scales in the second row. Figure 3.16A is the resultant WSS contour at Stokes Number of 1.0 (which is well below the fluid transition); Figure 3.16B is the resultant WSS contour at a Stokes Number of 3.2 (at the transition); Figure $3.16 \mathrm{C}$ is the resultant WSS contour at a Stokes Number of 10.0 (well above the transition). 
A

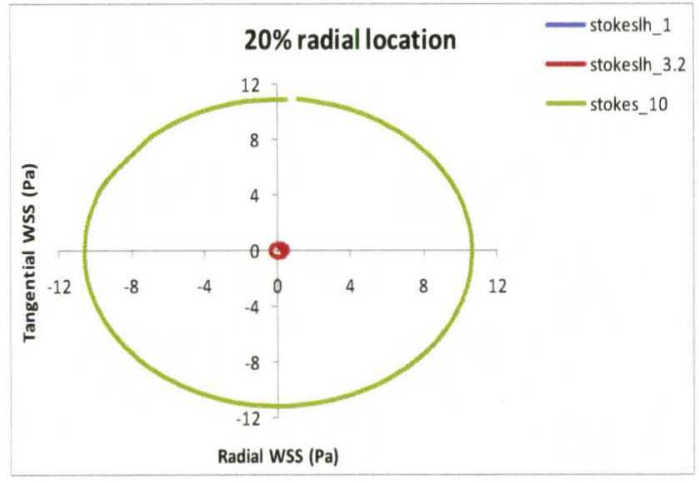

C

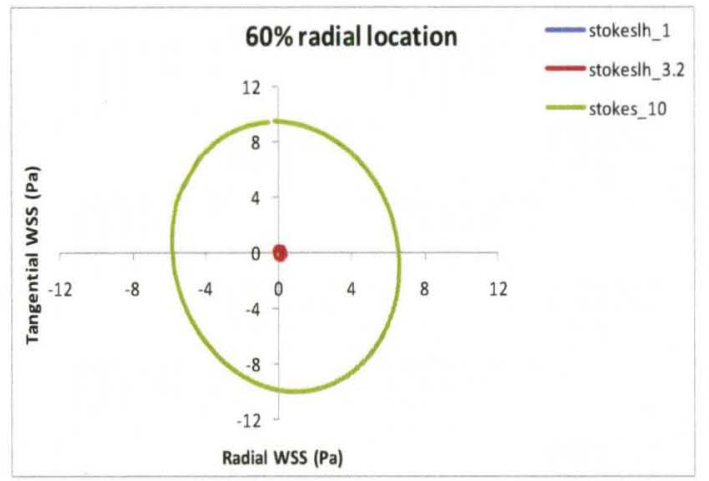

B

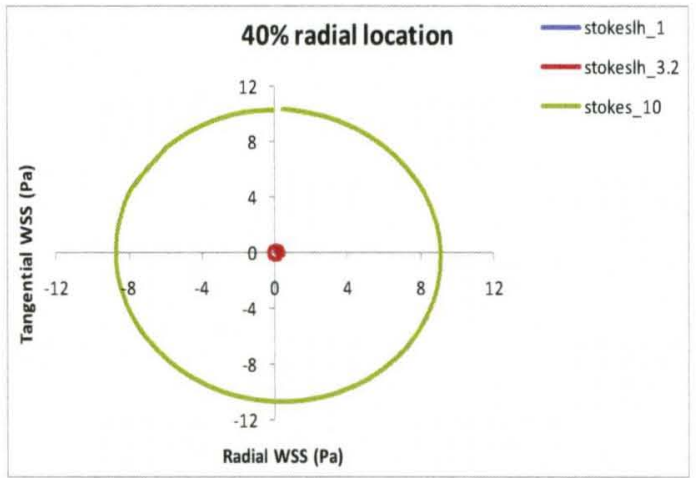

D

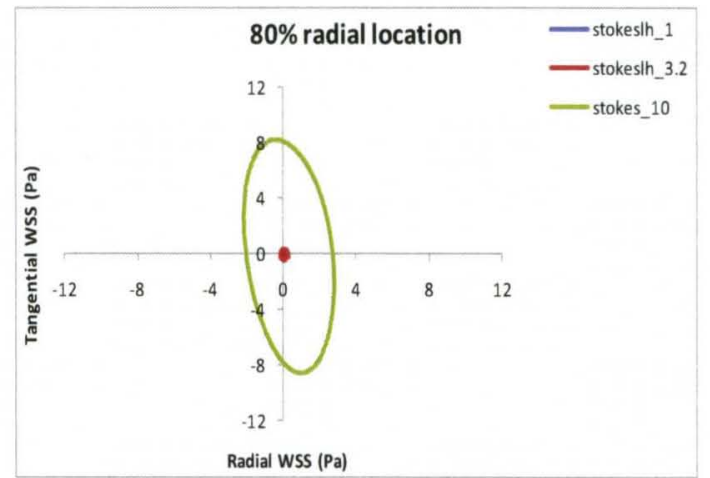

Figure 3.17. Tangential vs. radial WSS plots below, at, and above the transition at four radial locations for Set 6. Figure 3.17A, B, C, and D show plots at $20 \%, 40 \%, 60 \%$, and $80 \%$ radial locations, respectively. 


\subsubsection{CONCLUSION}

A comprehensive fluid dynamics study was performed in this chapter to understand the nature of oscillatory fluidic WSS as a function of three dimensionless flow parameters (Slope ratio, Stokes Number, and Froude Number). The main advantage of using the dimensional parameters was that it allowed a substantial reduction in computational effort by reducing the system properties from seven to three. This study encompassed how fluid behaves below the transition, at the transition, and above the transition for each of these parameters. The fluid transitions were determined from a free surface analysis for each of the three parameters. A study of the component WSS plots showed that the distribution and magnitude of WSS varies significantly depending on the value of each parameter relative to all the individual parameter transitions. Further, the nature of WSS component distributions at different radial locations was also determined. 


\section{CHAPTER FOUR}

\section{DIRECTIONAL OSCILLATORY SHEAR INDEX (DOSI) AND THE EFFECTS \\ OF DOSI AND SHEAR MAGNITUDES ON ENDOTHELIAL CELL \\ PROLIFERATION AND MORPHOLOGY}

\subsection{INTRODUCTION AND BACKGROUND}

Hemodynamic forces, especially fluidic wall shear stress (WSS) are a proven factor in affecting various cellular responses and gene expressions of anchored cells subjected to fluid flow. Previous documentation indicated that cellular responses under fluid flow may vary in different ways depending on the type of flow - steady unidirectional, oscillatory unidirectional, pulsatile unidirectional, and oscillatory bidirectional.

\subsubsection{Effects of steady unidirectional shear}

Steady unidirectional shear has significant impacts on various endothelial cellular responses and extensive studies were done to understand those effects. Yamaguchi, et al. (57) showed that steady wall shear stress directly affects the cell length and orientation. A 
three dimensional CFD model of endothelial cell shape was designed and simulated in this study. The model cell was capable of random rotation, deformation, migration and proliferation. The fluid flow was computed after each update of the cell shape with infinitesimal configuration changes and exerted wall shear stress was computed. The results showed that the model cells became confluent and were elongated and aligned with a shape index very close to the cells in vivo. Levesque, et al. (4) studied the elongation and orientation of endothelial (Bovine aortic endothelial cell) cells in response to shear stress. After growing the cells under the shear stress levels of 10,30 and 85 dynes $/ \mathrm{cm}^{2}$ using a parallel flow chamber, a computer based analysis was used to quantify the degree of cell elongation and orientation. The results in this study showed that the endothelial cells orient with the flow direction under influence of shear stress. There is a strong correlation between the degree of alignment and endothelial cell shape. Also endothelial cells become more elongated when exposed to higher shear stress. Inoguchi, et al. (30) determined the impact of gradually increased steady unidirectional shear stress on tissue morphological integrity, including cell shape and alignment. This study showed that gradually increased shear stress resulted in reduced cell detachment, a highly elongated cell shape and alignment of cells in the direction of the flow. Morphological responses of cultured bovine endothelial cells (ECs) exposed to steady unidirectional shear and hydrostatic pressure was investigated by Sugaya, et al. (73). They observed that shear exposed ECs were elongated and oriented in the direction of flow, whereas pressured ECs were also elongated, but randomly oriented. Chotard-Ghodsnia, et al. (8) studied the morphology of tumor cell/endothelial cell interactions under steady unidirectional shear flow by measuring the changes in cell area and cell aspect ratio for a 
range of shear stresses. They observed that axial spreading of cells was increased by shear stress, but radial spreading was unaffected. Sirois, et al. (9) studied the behavior of endothelial cells in the presence of erythrocytes in flow under steady shear stress. In this study, the projected area of cells was mostly smaller under dynamic conditions, particularly at a wall shear stress of $23 \mathrm{dyne} / \mathrm{cm}^{2}$. Fukushima, et al. (19) measured the surface topography of endothelial cells and wall shear stress distribution on the cell exposed to shear. In this study, a method of three dimensional cell shape measurement by confocal laser scanning microscopy and determination of shear stress distribution on the surface of the cells by particle tracking velocimetry with a scale-up model were presented. It has been shown here that the shear stress distribution on a cell has close correlation with the surface geometry and also with the surrounding cells and varied from cell to cell. The molecular basis of the effects of shear stress on vascular endothelial cells has been reviewed by $\mathrm{Li}$, et al. (74). In vitro study on cultured endothelial cells in flow channels here have been conducted to investigate the molecular mechanisms by which the cell converts the mechanical input into biochemical output, which leads to functional responses. Milovanova, et al. (75) worked on endothelial cell (seeded in an artificial capillary system) proliferation associated with abrupt reduction in shear stress of 5 dyne $/ \mathrm{cm}^{2}$. This study used flow cytometry to evaluate cell proliferation with ischemia and to determine the role of reactive oxygen species and apoptosis. The result of this experiment indicates reactive oxygen species dependent endothelial cell proliferation in a flow adapted microvascular endothelial cells as a response to ischemia and indicate that this response is not a consequence of apoptosis. Effects of long term shear stress exposure on calcium response and morphology of endothelial cells have been 
investigated by Kudo, et al. (18). Though shear stress (initially $2 \mathrm{~Pa}$ ) can induce intracellular calcium $\left[\mathrm{Ca}^{2+}\right]$ shortly (within 10 minutes) (about $27 \%$ of the cells exhibited calcium response) after initiation of flow, the effect of long term exposure ( $24 \mathrm{hr})$ of shear stress on $\left[\mathrm{Ca}^{2+}\right]$ responses is minimum (about $4 \%$ after 24 hours of exposure). Shear stress transmission to endothelial cells in blood vessels lined with an endothelial surface layer (ESL) has been studied by Secomb, et al. (76). They found some significant implications for studies of endothelial responses to shear stress- i) Transmission of shear stress to endothelial cells occurs almost entirely via attachment points of the ESL to the cell membrane. ii) The presence of ESL significantly alter the spatial variation of shear stress experienced by endothelial cells. Slater, et al. (77) investigated the shear (ranging from $0.2 \mathrm{dyne} / \mathrm{cm}^{2}$ to $2.3 \mathrm{dyne} / \mathrm{cm}^{2}$ ) effects on endothelial cells at hypothermic temperatures. As a result, it has been demonstrated that endothelial cell rounding increases with increasing shear during hypothermic machine perfusion preservation (MPP) and the cell rounding can be prevented by utilizing low flow rates. Sato, et al. (78) investigated the changes in endothelial cell (Bovine aortic endothelial cell) stiffness induced by shear stress (about $2 \mathrm{~Pa}$ or higher) and measured the changes by atomic force microscopy (AFM). As a result the cell shape became elongated to the flow direction and the location of nucleus moved to downstream side by shear flow. In this experiment, at 6 hour exposure, the stiffness was higher in the upstream side in a cell whereas after 24 hours of exposure, the stiffness became the same at both sides. Morphology and migration of individual endothelial cells cultured in a flow chamber under shear stress had been studied by Nagayama, et al. (20). The results of this effort indicate that the initial orientation and shape of cells affect the process of receiving the external shear 
stress (of about 50 dyne $/ \mathrm{cm}^{2}$ ) stimulus. So the external shear stimulus causes an organization of "the small group behavior party" due to mutual interaction of cells. Fernandez, et al. (79) investigated whether endothelial cells cultured on vascular grafts were able to transduce shear stress. It has been found that endothelial cells on fibrin coated small diameter ePTFE are mechanoresponsive to shear stress. Inoguchi, et al. (30) studied to determine whether gradually increased shear stress applied to HUVECs (Human umbilical vein endothelial cell) improves cell retention and tissue morphological integrity including cell shape and alignment, actin fiber alignment and vascular endothelial cadherin. A gradually increased graded exposure from low $\left(3.2 \mathrm{dyne} / \mathrm{cm}^{2}\right)$ to a high shear stress (19.6 dyne $\left./ \mathrm{cm}^{2}\right)$ in this experiment resulted in reduced cell detachment, a highly elongated cell shape and orientation or alignment of both cells and actin fibers which were parallel to flow direction. Zhao, et al. (80) showed that wall shear stress on the order of $10 \mathrm{dyne} / \mathrm{cm}^{2}$ can cause endothelial cells to align with and elongate in the direction of flow. In this study, it was found that the combination of shear stress and hoop stretch at physiologic levels evoked a different morphologic response than either of the effects alone. Further, they have shown that the alignment and elongation obtained when the cells were subjected to hoop stretch in addition to shear stress. Levesque, et al. (81) investigated the influence of monolayer age, serum content of the perfusing fluid and substrate on the response of an anchorage dependent mammalian cells exposed to shear stress using cultured bovine aortic cells. The differences in response to shear stress were observed for an ECM covered as compared to a bare substrate which, according to this study, might be important in comparing various laboratory studies and in designing optimum, and scale up of mammalian cell culture systems. Kudo, et al. (82) 
studied the effect of shear stress on macromolecules uptake into cultured endothelial cells, because abnormal uptake of macromolecules into an endothelial cell monolayer can cause several vascular diseases. In this effort, they found that the uptake of albumin into endothelial cells exposed to shear stress increased for a lower shear stress range and decreased for high shear stress. Grouping behavior of individual endothelial cells under shear stress has been studied by Tanishita, et al. (83). In this study, morphology and migration of individual cultured cells with wall shear stress of 3 and $5 \mathrm{~Pa}$ have been observed under an optical microscope. It was concluded from this study that the initial orientation and shape of the cells affect the process of receiving the external shear stress stimulus and also the behavior of the individual cell depends on geometry of surrounding cells. Feugier, et al. (23) examined the degree to which human umbilical venous endothelial cells (HUVECs) adhered to three materials (polymeric, vascular, and prosthesis) that had been coated with same extra cellular matrix proteins. After exposed to shear stress representative of femoro-distal bypass using a cone and plate shearing device, they quantified cell number, area of coverage and degree of cell spreading using image analysis techniques. Levesque, et al. (81) demonstrated that in response to fluid imposed laminar shear stress, cultured vascular endothelial cells undergo a dramatic alteration in structure and function. Bovine aortic endothelial cells (BAEC) have been exposed to shear in the range of 15 to $60 \mathrm{dyne} / \mathrm{cm}^{2}$ and it was reported that there was an elongation and orientation of endothelial cells exposed to shear stress. In the study of Chiu, et al. (29), a co-culture flow system was developed to study the effect of shear stress on interaction of endothelial cells (EC) and smooth muscle cells (SMC) separated by a porous membrane with only the EC side subjected to the flow condition. Different 
perpendicular orientations of SMC have been observed by exposing EC to different shear values. Hughes, et al. (84) described the effects of fluid shear stress, vascular endothelial growth factor (VEGF), and sphingosin 1-phosphate (S1P) on the migration of HUVECs. This work represented the initial steps toward predicting cell migration based upon the activation state of the receptors and enzymes involved in the chemokinetic response. Sato, et al. (10) studied the viscoelastic properties of cultured endothelial cells exposed to steady unidirectional shear stress (of about $2 \mathrm{~Pa}$ ), measured by a micropipette technique and then analyzed using a standard linear viscoelastic model. As a result, enhanced viscous properties were observed for the elongated cells, whereas spherical cells upon detachment, even those exposed to shear stress, did not show such significant changes in viscoelastic mechanical properties. Martin, et al. (85) monitored the shear induced cytosolic reactive oxygen species (ROS) production in human umbilical vein endothelial cells (HUVECs) using the oxidant-sensitive fluorescent probe 2,7dichlorodihydrofluoroscein diacetate (DCF-DA). Therefore they established a method to monitor lipid peroxidation in the membranes of sheared ECs and investigated the contribution of cytosolic ROS to membranous lipid peroxidation and to intracellular shear induced signaling. Tang, et al. (86) studied the shear stress distribution on the membrane of endothelial cells using 3-D computational modeling with fluid structure interaction. Effects of shear stress on permeability of the endothelial monolayer was investigated by Sakamoto, et al. (15) by using an endothelial cell (EC) and smooth muscle cell (SMC) co cultured model (CM). In this effort, the results suggested that the cellular interactions between EC's and SMC's have immense influence on EC permeability. 


\subsubsection{Effects of unidirectional oscillatory shear stress on cellular responses}

Effects of unidirectional oscillatory shear on cellular responses have also been investigated. Hsiai, et al. (87) found that monocytes displayed unique forward and backward motion, undergoing rolling, binding and dissociation with other monocytes in response to unidirectional oscillatory shear. In another study, Chappell, et al. (88) investigated the effects of unidirectional oscillatory shear stress on adhesion molecule expression in cultured human endothelium. The study revealed that unidirectional oscillatory shear stress can stimulate mononuclear leukocyte adhesion and migration into the arterial wall. Further, Ku, et al. (89) discovered that reversal of shear in primarily unidirectional pulsatile flow in the human carotid bifurcation has an important impact on atherosclerotic plaque formation. They developed an oscillatory shear index (OSI) to quantify the reversal of shear during pulsatile flow:

The scalar oscillatory shear index (OSI) is a measure of the reversal of shear in pulsatile flow.

$$
O S I=1-\frac{\left|\int_{0}^{T} \overrightarrow{\tau_{w}} d t\right|}{\int_{0}^{T}\left|\overrightarrow{\tau_{w}}\right| d t}
$$

where $\tau_{\mathrm{w}}$ is the instantaneous shear and $\mathrm{T}$ is the cycle period.

OSI is zero for monotonically positive shear, and unity for fully reversing (oscillatory) shear. OSI has been applied to flows that are largely unidirectional. In an orbiting dish, however, shear is strongly bidirectional, acting radially and tangentially. In their study of this pulsatile flow using a Y-shaped average human carotid bifurcation (Y-AHCB) model, they found that there was a strong and positive correlation between the OSI and 
intimal wall thickness at the outer and inner walls of the carotid sinus, though the correlation was weak at the side walls of the sinus. Further continuation of this study was done by Ding, et al. (90). The objectives of the study were to examine whether the extent of this correlation (strong or weak) lies in the deviation of geometry of Y-AHCB from real human carotid bifurcation, and whether this can be improved in the tuning fork shaped model of the average human carotid bifurcation (TF-AHCB). Intimal thickness was measured by analyzing seventy-four carotid bifurcation specimens in both $\mathrm{Y}$ and TF shaped models. The observation and statistical analysis however showed that TF-shaped model was much closer to the average shape of a real human carotid bifurcation. Furthermore, the pulsatile flow measurements in those two models confirmed that the OSI values at the side wall of the sinus of the TF-shaped model were more than two times larger than those in the $\mathrm{Y}$-shaped model. Remembering the previous study of $\mathrm{Ku}$, et al. (89), this confirmed that there is stronger positive correlation between OSI and intimal thickness in the tuning fork geometry indicating that the TF-shaped model is a significant improvement over the traditional Y-shaped model. Hwang, et al. (91) examined the hypothesis that oscillatory shear (of about $5 \mathrm{dynes} / \mathrm{cm}^{2}$ ) and laminar shear (of about 15 dynes $/ \mathrm{cm}^{2}$ ) differently regulate production of $\mathrm{O}_{2}^{-}$differently from endothelial $\mathrm{NAD}(\mathrm{P}) \mathrm{H}$ oxidates, which in turn is responsible for their opposite effects on a critical atherogenic event, monocyte adhesion. The result suggested that chronic exposure of endothelial cells to oscillatory shear stimulates $\mathrm{O}_{2}{ }^{-}$and or its derivatives produced from $\mathrm{p} 47^{\text {phox }}$-dependent $\mathrm{NAD}(\mathrm{P}) \mathrm{H}$ oxidase, which in turn leads to monocyte adhesion, an early and critical atherogenic event. Han, et al. (92) examined the response surface structural layers when steady or oscillatory shear was applied at their outer edge. The results showed that the 
deflection of structural elements was proportional to the product of applied steady shear stress and their length and inversely proportional to the natural damped vibration frequency of the structural element. In the case of oscillatory shear, they found that the motion of both the fluid and structural elements are in a quasi-steady state in physiological conditions. In another study, Schmidt, et al. (93) discussed the consequences of an oscillatory fluid shear mechanism on nutrient transport in bone during physical activity and ultrasonic therapy. A transient model was derived here from oscillatory Taylor-Aris dispersion phenomena which was used to predict a ratio of effective-to-molecular diffusivity. This model was expected to be useful for understanding differences in bone growth as a function of type of movement or to develop new physical therapies.

\subsubsection{Unidirectional pulsatile shear effects}

Pulsatility, which occurs throughout most of the arterial system, has also been shown to influence cell responses. Owatverot, et al. (25) found that unidirectional pulsatile shear stress on cultured human aortic endothelial cells was a stronger stimulus than steady shear for inducing cell orientation. It was observed that pure uniaxial cyclic strain of $2 \%$ at $0.5 \mathrm{~Hz}$, shear stress at $80 \mathrm{dyne} / \mathrm{cm}^{2}$ at $0.5 \mathrm{~Hz}$, produce the same time course of cell reorientation. This result suggests that the magnitude and direction of cyclic strain and fluid shear are important determinants, both individually and collectively of endothelial cell response. In another effort, Hsiai, et al. (94) found that pulsatility significantly increased the rates at which endothelial cells elongated and realigned, compared to steady flow. Bovine aortic endothelial cells (BAECs) monolayers 
were exposed here under pulsatile flow at high $\left(293 \mathrm{dyne} / \mathrm{cm}^{2}\right)$ and low $\left(71 \mathrm{dyne} / \mathrm{cm}^{2}\right)$ slew rate under steady laminar flow at a shear stress of $50 \mathrm{dyne} / \mathrm{cm}^{2}$. It was observed that pulsatile flow significantly increased the rate at zero slew rate, furthermore, EC remodeling is faster in response to high than to low slew rates at a given shear value.

While all of the studies above used laminar flow, a few investigations have been reported on the effects of turbulent shear stress on cellular responses. Davis, et al. (95), for instance, compared the effects of laminar and turbulent shear stress on vascular endothelial cells. The results indicated that unsteady blood flow characteristics, rather than magnitude of wall shear stress, are the major determinant of shear-induced endothelial turnover. In another study of $\mathrm{He}$, et al. (96), the pulsatile hemodynamic of the left coronary artery bifurcation was numerically simulated using the spectral element method for realistic in vitro anatomic and physiologic conditions. Results indicated that arterial wall shear stress was significantly lower in the bifurcation region, including the side walls and the greatest oscillatory behavior was localized to the outer wall of the circumflex artery. Milovanova, et al. (97) investigated whether the lung endothelial cell proliferation with decreased shear stress is mediated by reactive oxygen species. Frangos, et al. (98) developed a flow apparatus to study the metabolic response of anchorage dependent cells (HUVEC) to a wide range of steady and pulsatile shear stresses (of about $24 \mathrm{dyne} / \mathrm{cm}^{2}$ ) under well controlled conditions. According to this study, metabolite production can be maximized by maximizing shear stresses. Fisher, et al. (99) presented the recent information concerning endothelial cell responses to shear stress associated with blood flow. Himburg, et al (100) studied the frequency dependent response of the vascular endothelium to pulsatile shear stress. This study suggests that the arterial regions 
subject to both shear reversal and dominant frequencies that exceed normal heart rates cause greater risk for atherosclerotic lesion development. Hwang, et al. (101) further studied the effects of pulsatile and oscillatory shear stress on NADPH oxidase subunit expression. Bovine aortic endothelial cells (BAECs) in the presence of $50 \mu \mathrm{g} / \mathrm{ml}$ of native LDL (low-density lipoprotein) were exposed in this study to pulsatile and oscillatory flow. The results showed that oscillatory flow induced greater oxidative stress by $\mathrm{O}_{2}{ }^{-}$ production and thus enhances LDL oxidation and upregulation of inflammatory markers. On the other hand, pulsatile flow favored upregulation of atheroprotective and antioxidant genes that prevent LDL oxidation. Bacabac, et al. (102) investigated bone cell responses to vibration stress at a wide frequency range $(5-100 \mathrm{~Hz})$. They used NO and prostaglandin $\mathrm{E}_{2}\left(\mathrm{PGE}_{2}\right)$ release, and $\mathrm{COX}-2$ mRNA expressions as parameters for bone cell response since these molecules regulate bone adaptation to mechanical loading. The results indicated that there was a correlation between $\mathrm{NO}$ and $\mathrm{PGE}_{2}$ and the correlation relates to nucleus oscillations, providing a physical basis for cellular mechanosensing of high frequency loading. While the impacts of steady and oscillatory shear on endothelial cell responses have been previously observed, these results demonstrate that the directionality of shear also affects cellular responses. 


\subsection{MATERIALS AND METHODS}

\subsubsection{CFD Methodology and case set up procedure}

Details of CFD methodology were described in Chapter 2. The simulated cases mirrored those of the experiments that used a 3-D Petri dish with height of $1.5 \mathrm{~cm}$ and radius of $2.5 \mathrm{~cm}$. A mesh with 300,000 computational cells was applied to the volume of the dish. The cell count was optimized in a previous study (103). The cell culture medium, which is an incompressible fluid, was simulated as a liquid with density of $997.3 \mathrm{~kg} / \mathrm{m}^{3}$ and viscosity of $0.00072 \mathrm{~kg} / \mathrm{m}$-s. The static liquid height was set at $0.2 \mathrm{~cm}$. The air above the cell culture medium was simulated as a gas with density of $1.225 \mathrm{~kg} / \mathrm{m}^{3}$ and viscosity of $1.7894 \mathrm{E}-5 \mathrm{~kg} / \mathrm{m}-\mathrm{s}$. No slip boundary conditions were set at the bottom, vertical walls, and top (since the Petri-dish was covered by a lid) of the dish. The velocity generated throughout the orbit by the fluid in the dish can be defined as:

$$
\vec{V}=a^{*} \omega^{*} \exp \left(i * \omega^{*} t\right)
$$

where $\vec{V}=$ velocity of the fluid across the orbit, $\mathrm{a}=$ orbital radius, $\omega=$ angular velocity, $\mathrm{t}$ $=$ time

A time step of $0.001 \mathrm{~s}$ was used as a compromise between temporal resolution and computer time. The solution was carried out for several orbits to achieve periodic steady state. The residual values for continuity and $\mathrm{x}, \mathrm{y}$, and $\mathrm{z}$ velocities were set to 0.0001 , which was determined in preliminary tests to give rapid convergence without affecting the results. Orbital radius was $0.95 \mathrm{~cm}$ and orbital speeds of 50, 100 and 150 rpm were tested. The dimensionless parameters corresponding to those experimental cases appear in Appendix D. 


\subsubsection{Experimental protocol}

Endothelial cells were obtained from human umbilical veins (HUVECs) and were maintained in $10 \mathrm{ml}$ culture medium (M-199) including $10 \%$ fetal bovine serum, $10 \mathrm{ml}$ growth factor, $1 \mathrm{ml}$ of heparin and $5 \mathrm{ml}$ of Penicillin Streptomycin $(10000 \mu \mathrm{g} / \mathrm{ml})$ in Fibronectin-coated $(25 \mu \mathrm{g} / \mathrm{ml})$ cell culture dishes for 3 days at $37{ }^{\circ} \mathrm{C}, 5 \% \mathrm{CO}_{2}$ in a humidified $(80 \%)$ incubator. The cells were then plated at a density of $0.20 \mathrm{million} / \mathrm{ml}$ in new culture dishes ( $5 \mathrm{~cm}$ diameter $\times 1.5 \mathrm{~cm}$ height) containing $4 \mathrm{ml}$ of growth medium and allowed to adhere to the plate. The dishes then were placed on an orbital shaker platform (Model-3500, Thomas Scientific, Swedesboro, NJ, USA) at specified orbital speed to generate pulsatile shear stress on the cells. Another set of HUVECs were grown in stationary dishes. Another set of HUVECs were grown in stationary dishes. 


\subsubsection{Microscopy and image analysis}

Images of cells were captured by a Nikon inverted microscope eclipse TE 300 (Nikon Instruments Inc. Melville, NY, USA) at 10X magnification. All the images were captured by cool snap HQ digital B/W CCD (Roper Scientific, Trenton, NJ, USA) camera. Cells in those images were counted manually in Metamorph 4.6r5 software (Universal Imaging, Ypsilanti, MI, USA). The number of cells depends on each captured cell image frame area over which they were counted. The cell counts were then averaged over the three trials to quantify proliferation. To observe morphological changes, cell areas were measured by using region measurement tools in the same Metamorph software. Shape indices were then calculated and compared between exposed and unexposed cells. Proliferation and morphological indices were normalized by that of the stationary cells, which were considered controls.

\subsubsection{Cell shape index}

Shape index (SI) was defined as (104)

$$
S I=\frac{4^{*} \pi^{*} A}{P^{2}}
$$

Where $\mathrm{A}=$ area and $\mathrm{P}=$ perimeter of a single cell.

A circular cell has shape index of SI $=1$ while an infinitely elongated cell has a shape index of $\mathrm{SI}=0$. 


\subsubsection{Directional Oscillatory Shear Index (DOSI)}

The scalar oscillatory shear index (OSI) (89) is a measure of the reversal of shear in pulsatile flow.

$$
O S I=1-\frac{\left|\int_{0}^{T} \overrightarrow{\tau_{w}} d t\right|}{\int_{0}^{T}\left|\overrightarrow{\tau_{w}}\right| d t}
$$

where $\tau_{w}$ is the instantaneous shear and $T$ is the cycle period. OSI is zero for monotonically positive shear, and unity for fully reversing (oscillatory) shear. OSI has been applied to flows that are largely unidirectional. In an orbiting dish, however, shear is strongly bidirectional, acting radially and tangentially. Thus a new directional oscillatory shear index (DOSI) was formulated to quantify the dominance of one direction of shear oscillation over another

$$
\mathrm{DOSI}=1-\frac{\mathrm{OSI}_{2}}{\mathrm{OSI}_{1}}
$$

where

$$
\begin{aligned}
& O S I_{1}=1-\frac{\left|\int_{0}^{T} \tau_{1} d t\right|}{\int_{0}^{\tau_{1}}\left|\vec{\tau}_{n}\right| d t} \\
& O S I_{2}=1-\frac{\left|\int_{0}^{T} \tau_{2} d t\right|}{\int_{0}^{T}\left|\vec{\tau}_{w}\right| d t}
\end{aligned}
$$

Where direction 1 and 2 are defined such that

$$
\int_{0}^{T} \tau_{1} \tau_{2} d t=0
$$


Direction 1 is assigned as the direction of the larger of the two oscillations, and $\overrightarrow{\tau_{w}}$ is for this case a two-dimensional vector. Directions 1 and 2 are analogous to principal axes for the full cycle of periodic two-dimensional shear. DOSI is unity for unidirectional flow for any nonzero amount of shear reversal, and zero for oscillations of equal magnitudes in both directions. Though $\mathrm{OSI}_{1}$ appears in the denominator of equation 4.5 , since $\mathrm{OSI}_{1}>$ $\mathrm{OSI}_{2}$, DOSI remains well-behaved as $\mathrm{OSI}_{1}$ and $\mathrm{OSI}_{2}$ approach zero.

\subsubsection{Statistical methods}

Unpaired t-tests to determine statistical significance compared with controls were performed using Graph Pad Prism Software (Graph Pad Software, La Jolla, CA, USA). Data are statistically significant when $\mathrm{p}<0.05$ and insignificant data are denoted by $\mathrm{p}=\mathrm{NS}$ (Not Significant) in result section.

Four different statistical distribution models were used to identify the individual and interacting effects of DOSI, shear magnitudes and orbital speeds on cellular responses, including cell proliferation, average cell area, cell shape index, angle of cell orientation and gene expression. These models are discussed in sections 4.2.6.1 -4.2.6.4.

\subsubsection{Poisson distribution}

Cell proliferation is a discrete random variable that includes only positive integers, hence a Poisson distribution (105-107) was used to model this dataset. Cell proliferation at different DOSI, shear magnitude and orbital speeds were compared with log-linear regression models under a Poisson family with R 2.11.0 software (Bell Laboratories, Alcatel-Lucent, Paris, France). The GLM (generalized linear model) 
command was used to get the parameter estimates and p-value of the null hypothesis comparing stressed cells to unstressed controls. Generalized linear regression under the Poisson family was used to evaluate the individual and interaction effects of multiple factors (DOSI, shear magnitude and orbital speed) on cell proliferation.

The probability of the Poisson distribution of random variable $\mathrm{Y}$ to have integer value y can be expressed as:

$$
P_{r}\{Y=y\}=\frac{e^{-\mu} \mu^{y}}{y !}
$$

where parameter $\mu$ is greater than zero.

For $\mu>0$, the mean and variance of this distribution are

$$
E(Y)=\operatorname{var}(Y)=\mu
$$

Since the mean is equal to the variance, any factor that affects one will also affect another.

The generalized log linear regression model of a response $\mathrm{Y}$ on three covariates $\mathrm{x}_{1}, \mathrm{x}_{2}$ and $\mathrm{x}_{3}$ with the canonical log link function is given by

$$
\ln \left(Y_{i}\right)=\beta_{0}+x_{1 i} \beta_{1}+x_{2 i} \beta_{2}+x_{3 i} \beta_{3}+\varepsilon_{i}
$$

where $Y$ is the random variable or response, $x$ is the vector of explanatory variables, $\beta_{i}$ are regression coefficients, and $i=1,2, \ldots . n$, where $n$ is the number of observations under consideration and $\varepsilon_{i}$ is the random error component corresponding to the $\mathrm{i}$-th observation.

Goodness of fit for multi-factorial models was determined by deviance, which describes the difference between the model predictions and the measurements. For Poisson response, deviance is of the form 


$$
D=2 \sum\left\{y_{i} \log \left(\frac{y_{i}}{\hat{\mu}_{i}}\right)-\left(y_{i}-\hat{\mu}_{i}\right)\right\}
$$

where $\hat{\mu}_{i}$ denotes the fitted values based on the current parameter estimates. The first term represents binomial deviances (twice a sum of observed times log observed fitted data) and the second term represents the sum of differences between observed and fitted values. For large samples, the distribution of the deviance is chi-squared with n-p degrees of freedom, where $\mathrm{n}$ is the number of observations and $\mathrm{p}$ is the number of parameters.

\subsubsection{Gaussian distribution}

While the histogram of cell shape index (SI) exhibited positive skewing (Figure 4.1A), preliminary tests indicated that a symmetrical Gaussian model provided a better fit than an asymmetrical gamma model. Therefore, a Gaussian distribution was used to model this parameter. The probability density function of the Gaussian (normal) random variable can be written as

$$
f\left(x ; \mu, \sigma^{2}\right)=\frac{1}{\sigma \sqrt{2 \pi}} e^{\frac{(x-\mu)^{2}}{2 \sigma^{2}}}
$$

Where $\mathrm{x}, \mu \in \mathfrak{R}$ and $\sigma>0 . \mu$ and $\sigma^{2}$ are the two parameters that characterize this distribution.

The generalized linear regression model (with the canonical identity link) of a response $\mathrm{Y}$ on three covariates $\mathrm{x}_{1}, \mathrm{x}_{2}, \mathrm{x}_{3}$ can be written as

$$
Y_{i}=\beta_{0}+x_{1 i} \beta_{1}+x_{2 i} \beta_{2}+x_{3 i} \beta_{3}+\varepsilon_{i}
$$




\subsubsection{Gamma distribution}

The histograms of cell area (Figure 4.1B) exhibited positive skewing, hence Gamma distributions were used (105-107) to model these responses. The probability density function for a Gamma random variable $\mathrm{x}$ is given by

$$
f(x ; k, \theta)=x^{k-1} \frac{e^{-x / \theta}}{\theta^{k} \Gamma(k)}
$$

where $\mathrm{x}>0$ and $\mathrm{k}, \theta>0$.

Under this model, the generalized inverse linear regression model of a response $\mathrm{Y}$ can be expressed as:

$$
\frac{1}{Y_{i}}=\beta_{0}+x_{1 i} \beta_{1}+x_{2 i} \beta_{2}+x_{3 i} \beta_{3}+\varepsilon_{1}
$$

\subsubsection{Circular linear regression}

Cell orientation angle is a circular dependent variable, i.e., it has values on a periodic scale on which the end is the same as the beginning (Figure 4.1C). On the other hand, the independent variables DOSI, shear magnitude and orbital speed have monotonic (linear) scales. The circular-linear regression method (108-112) therefore was used to determine the effects of the three covariates DOSI, shear magnitude and orbital speed on the angle of cell orientation.

The standard way of doing this is to regress the response on the covariates. But the problem is that the measurement scales for the response and the covariates are fundamentally different from each other. 
The concept of statistical variation of an angle is realized through the assignment of different values to it as we move the radius vector along the circumference of a circle in a specific sense (clockwise or counter-clockwise) and basically represents the randomness in a population of several directions. The data on such a variable actually constitute such directions which are mathematically quantified by measuring the angle with respect to a particular reference axis. So the concept of angle intrinsically represents a direction and hence defines it as a circular random variable. On the other hand the covariates take real values and the data in this case represent their magnitudes. Thus they are non-directional variables and hence are linear is nature. Due to structural difference between the response and covariates, standard linear regression will not work here; rather, a linear transform of the angle of cell orientation can be regressed on the linear covariates. This is popularly known as the circular-linear regression. Such a regression model was considered here for learning the response .From figure $4.1 \mathrm{C}$ it is observed that the frequency distribution of the angle of cell orientation resembles a circular normal model. This fact can be used to perform the intended circular - linear regression in this case. To start with we define the circular normal distribution.

Let us denote angle of cell orientation, DOSI, Shear magnitude, and Orbital speed by $\Theta, D, M$ and $S$ respectively. A circular variable $\Theta$ is said to follow a Circular Normal distribution $(\mathrm{CN})$ with mean parameter $\mu$ and concentration parameter $\kappa$ if its probability density function is given by

$$
f(\theta, \mu, \kappa)=\frac{1}{2 \pi I_{0}(\kappa)} e^{\kappa \cos (\theta-\mu)}
$$


Let

$$
\theta \mid(D, M, S)=(d, m, s) \sim C N(\mu, \kappa)
$$

Now the regression model is defined as

$$
\mu=\mu_{0}+g\left(\beta^{*} x\right)
$$

where $g$ is the link function and $x=(d, m, s) . \mu_{0}$. Here $\mu_{0}$ is the intercept and $\beta$ is the vector of regression coefficient corresponding to $\mathrm{D}, \mathrm{M}$ and $\mathrm{S}$ respectively. In this analysis $\mathrm{g}$ is taken as the two times of arc-tan function. Thus the final regression model is

$$
\theta=\mu_{0}+2 \arctan \left(\beta^{*} x\right)
$$

The goal is to estimate $\mu_{0}$ and $\beta$ by the method of maximum likelihood (ML) estimation. The analysis was done in the "circular" package of $\mathrm{R}$ and the detailed results along with their interpretations were reported in the result section. 
A.

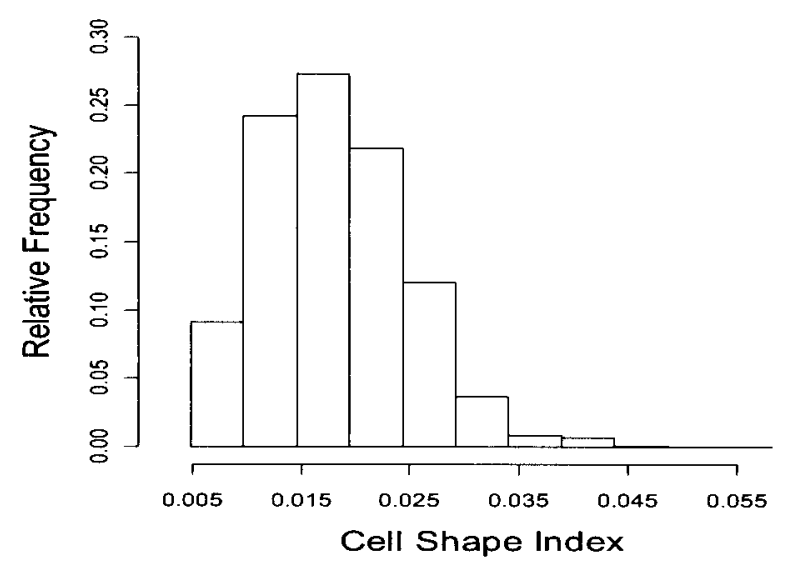

B.

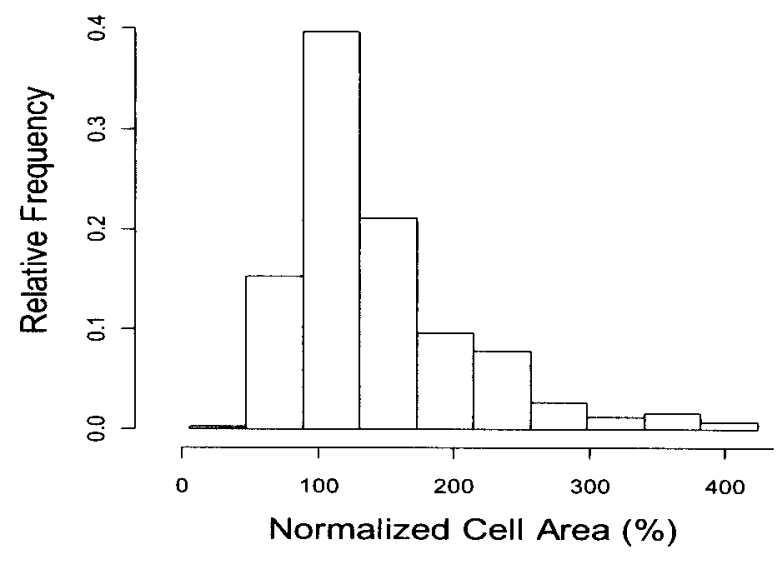

C.

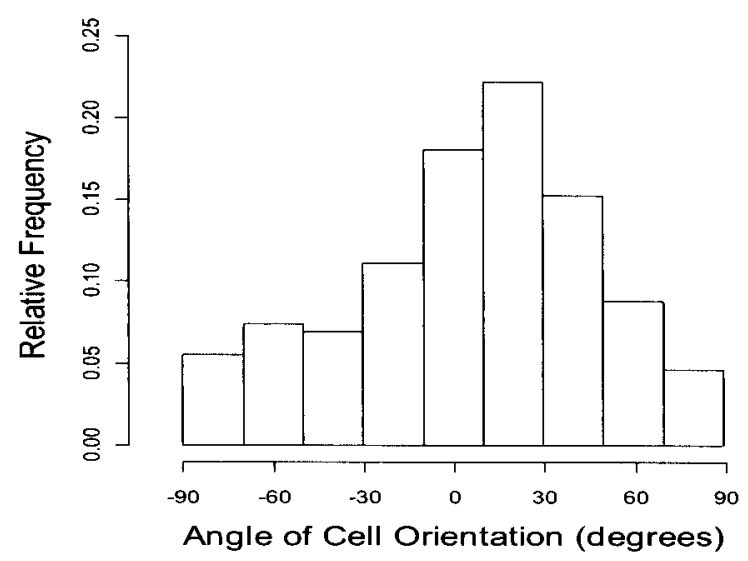

Figure 4.1. Frequency density distributions of cell shape index (Figure 4.1A), normalized cell area (\%) (Figure 4.1B) and angle of cell orientation (degrees) (Figure 4.1C). 


\subsection{RESULTS AND DISCUSSION}

\subsubsection{Determination of wall shear stress by CFD}

Resultant WSS was low and more or less uniform at low orbital speed (50 rpm), whereas it was higher and more nonuniform for higher orbital speed (100 and $150 \mathrm{rpm})$. The highest WSS occurred in a crescent-shaped area near the vertical wall under the deep part of the wave, and lower shear on the opposite side (Figure 4.2). At the lowest orbital speed (50 rpm), WSS was relatively constant over the cycle irrespective of dish location (Figure 4.2A), whereas shear varied throughout the cycle and differed with radial location at higher orbital speeds (100 and $150 \mathrm{rpm}$, Figure 4.2B, 4.2C). At $100 \mathrm{rpm}$, WSS ranges were $0-9 \mathrm{dyn} / \mathrm{cm}^{2}$ at $85 \%$ of the radius, $0-5 \mathrm{dyn} / \mathrm{cm}^{2}$ at $60 \%$ and $0-3 \mathrm{dyne} / \mathrm{cm}^{2}$ at $20 \%$, respectively, whereas at $150 \mathrm{rpm}$, WSS ranges were $0-12 \mathrm{dyn} / \mathrm{cm}^{2}, 0-8 \mathrm{dyn} / \mathrm{cm}^{2}$ and $0-4$ dyn $/ \mathrm{cm}^{2}$, respectively, at radial locations of $85 \%, 60 \%$ and $20 \%$. Root-mean square (RMS) values of these shear waveforms (Figure 4.2) showed similar trends, with RMS shear remaining relatively constant across the dish at $50 \mathrm{rpm}$, but increasing with radial location for the two higher orbital speeds (Table 4.1). Further, the extent of bi-directional flow reversals is illustrated in Figure 4.3 at different radial locations for different orbital speeds. The radial and tangential shear waveforms were nearly identical at the three different locations at $50 \mathrm{rpm}$ (Figure 4.3A) demonstrating uniform shear (both tangential and radial) exposure at low orbital speed. For higher orbital speeds of 100 and $150 \mathrm{rpm}$ (Figure 4.3B and 4.3C), radial shear waveforms fall in lower magnitude ranges (0-3 $\mathrm{dyn} / \mathrm{cm}^{2}$ for $100 \mathrm{rpm}$ and $-2-4 \mathrm{dyn} / \mathrm{cm}^{2}$ for $150 \mathrm{rpm}$ ) for all three radial locations, 
whereas the tangential shear waveforms were significantly higher near the edge (at $85 \%$ location) compared to that near the center ( $20 \%$ location) of the dish.

Table 4.1. RMS values of wall shear stress at different orbital speeds and radial locations.

\begin{tabular}{ccc}
\hline Orbital speed (rpm) & Radial location (\%) & RMS value of WSS $\left(\mathrm{dyn} / \mathrm{cm}^{2}\right)$ \\
\hline \multirow{2}{*}{50} & 20 & 0.550 \\
& 60 & 0.493 \\
& 85 & 0.425 \\
\hline \multirow{2}{*}{100} & 20 & 2.33 \\
& 60 & 3.41 \\
\hline \multirow{2}{*}{150} & 85 & 4.80 \\
\hline
\end{tabular}



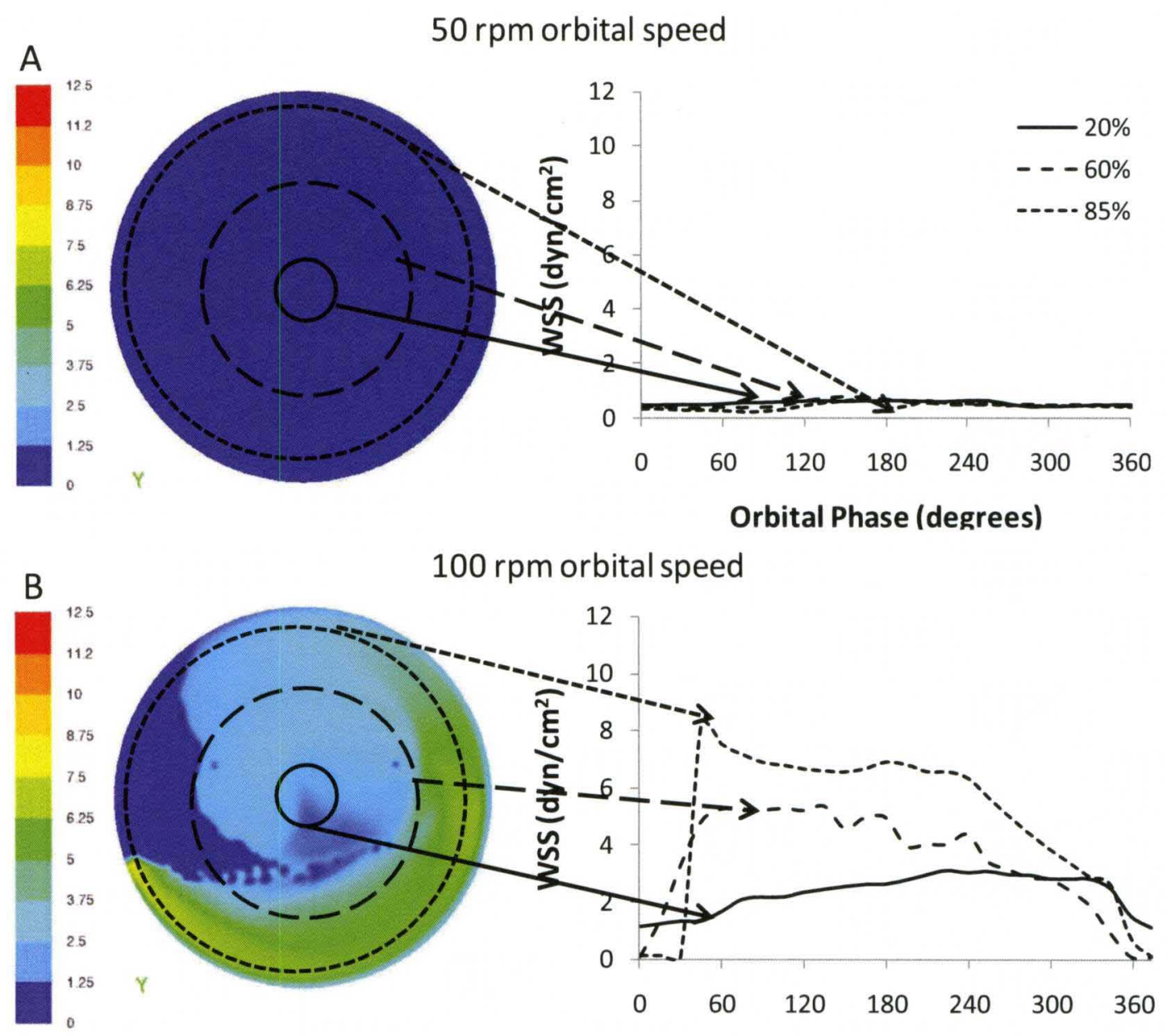

100 rpm orbital speed

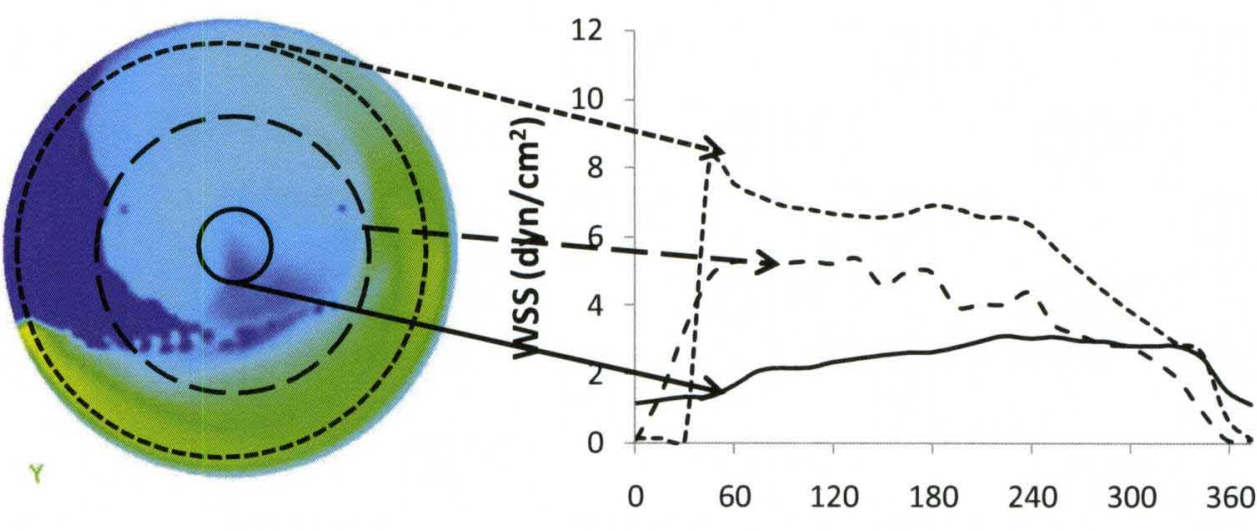

Orbital Phase (degrees)
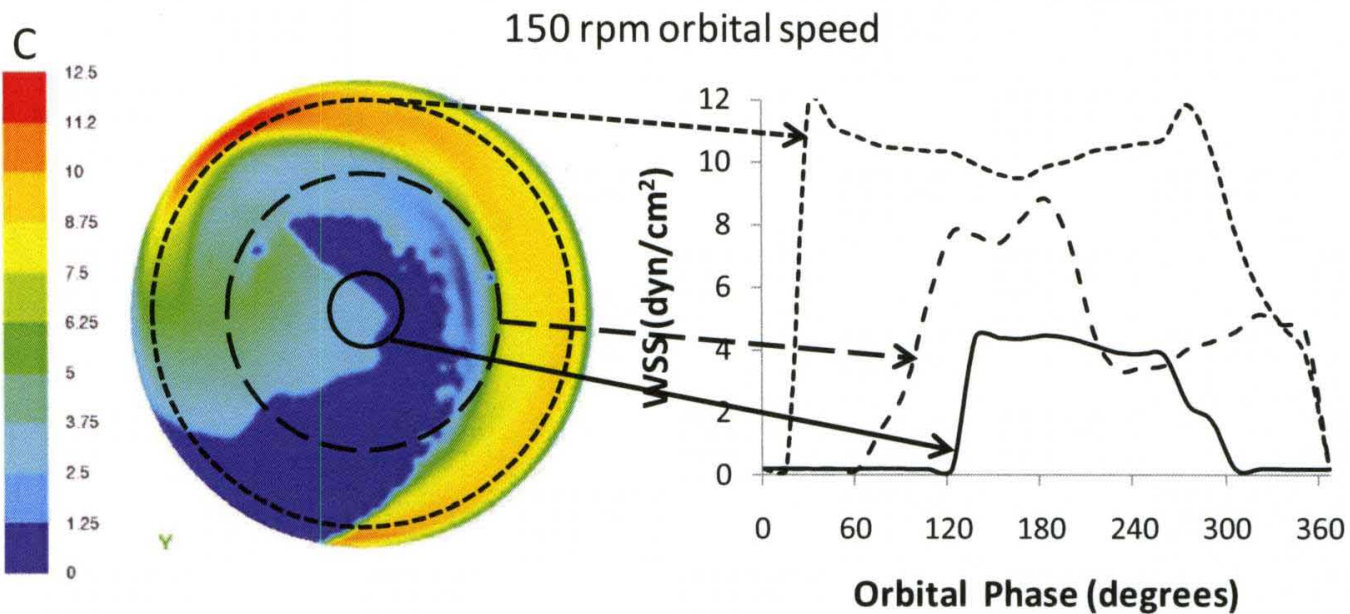

Figure 4.2. Resultant wall shear stress contours $\left(\mathrm{dyn} / \mathrm{cm}^{2}\right)$ viewed from below the dish and corresponding resultant shear waveforms at different radial locations $(20 \%, 60 \%$ and $85 \%$ ) and orbital speeds (50 rpm, $100 \mathrm{rpm}$ and $150 \mathrm{rpm})$. 
A.

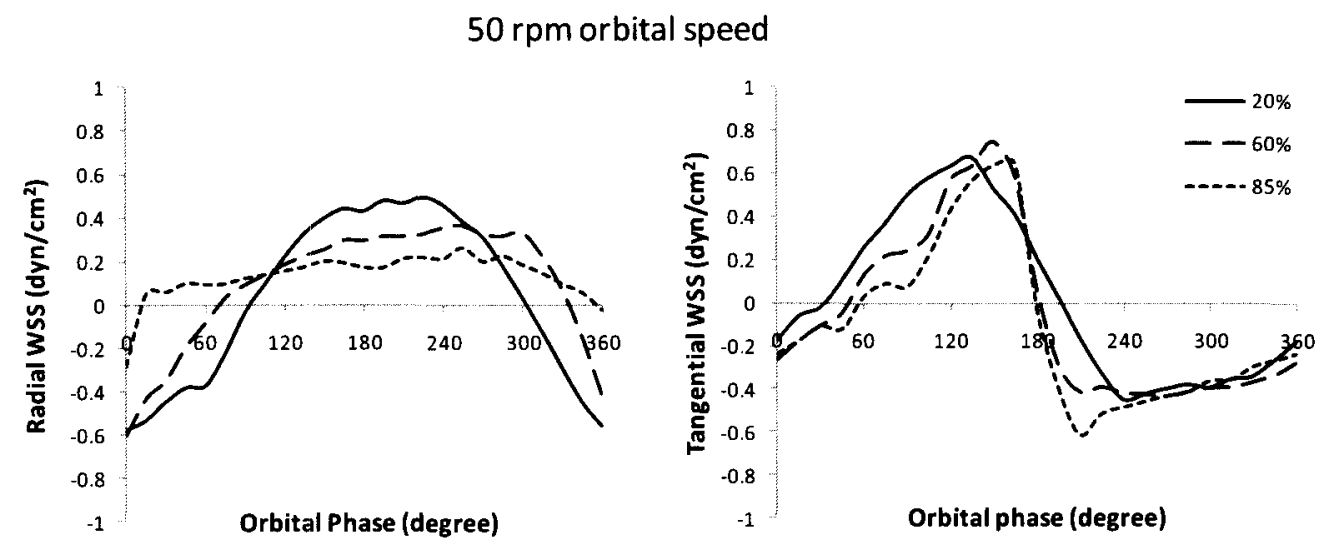

B.

$100 \mathrm{rpm}$ orbital speed
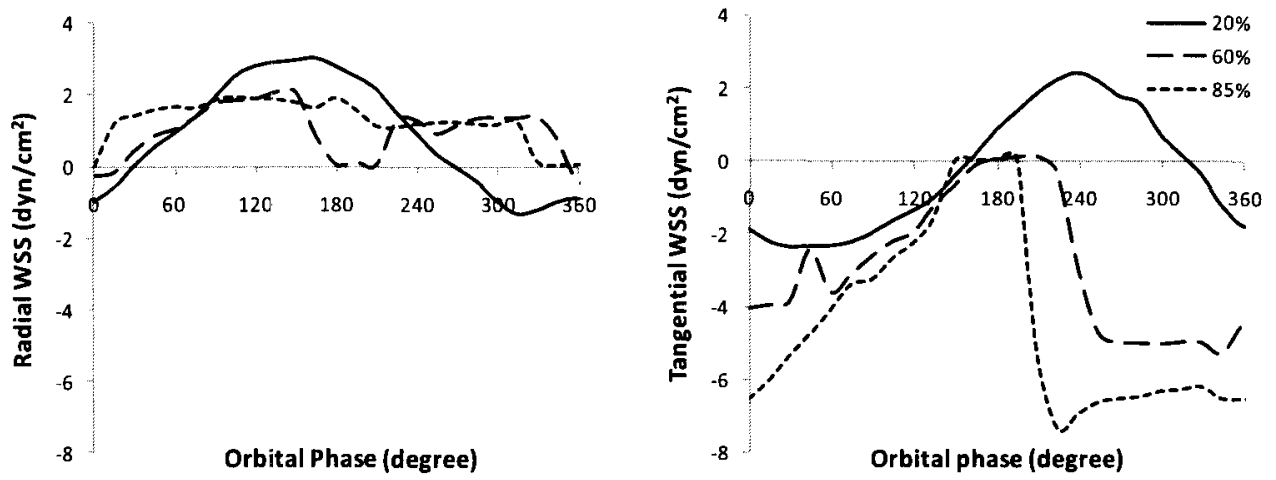

C.
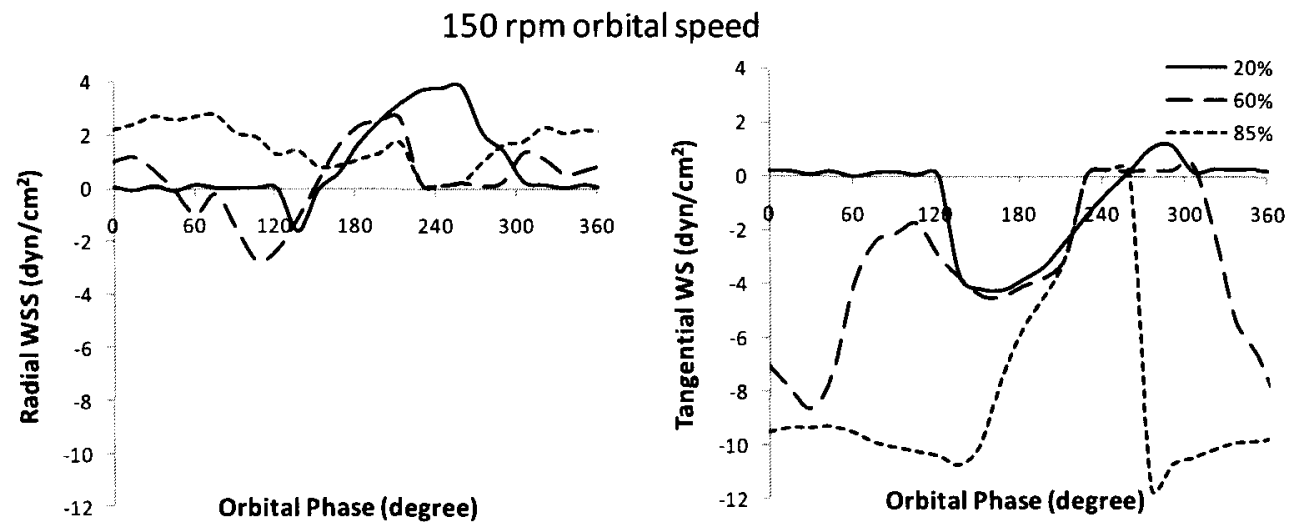

Figure 4.3. Bidirectional shear waveforms (radial and tangential) at different radial locations for different orbital speeds. 


\subsubsection{Determination of directional oscillatory shear index (DOSI)}

An example plot of the radial and tangential components of WSS extracted from the CFD results at 24 evenly spaced times throughout one complete orbit is shown in Figure 4.4 for the lowest orbital speed. Note that the shear transitions from being mostly radial at $20 \%$ of the radius to mostly tangential at $85 \%$, consistent with the blockage of radial flow presented by the vertical sides of the dish. There was strong reversal of shear in both directions. To determine DOSI, it was important to identify the principal axes. The axes were determined by linear curve fitting of those two WSS components for one complete cycle. The first principal direction was defined as the direction of the resultant of the two (radial and tangential) components. The second principal direction is perpendicular to the first. An example plot of WSS in terms $1^{\text {st }}$ principal direction vs. $2^{\text {nd }}$ principal direction at different radial locations for an orbital speed of $50 \mathrm{rpm}$ is shown in Figure 4.5. The DOSI values were then tabulated in (Table 4.2). At radial location of $20 \%$ for all speeds and at all radial locations for $50 \mathrm{rpm}$, shear oscillated nearly the same in both directions, producing low DOSI (Figure 4.6A, 4.6B, 4.6C). However, at increasing radius for the two higher speeds, tangential shear was less oscillatory and radial shear was more oscillatory, causing DOSI to be dramatically higher. For the two higher speeds at $85 \%$ of the radius, radial OSI was an order of magnitude larger than tangential OSI. 


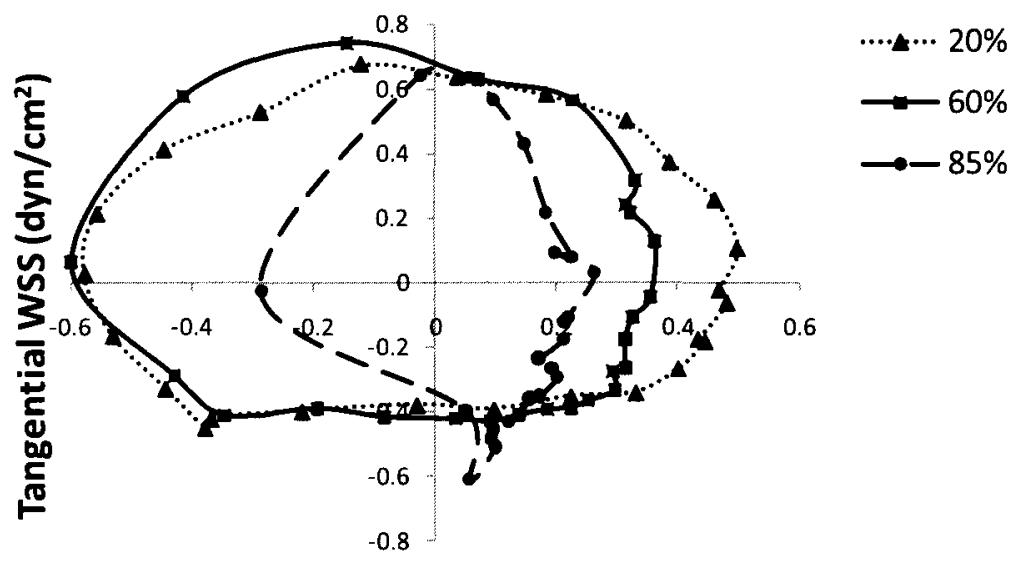

\section{Radial WSS (dyn/ $\mathrm{cm}^{2}$ )}

Figure 4.4. An example plot of tangential versus radial wall shear stress at different radial locations $(20 \%, 60 \%$, and $85 \%)$ at $50 \mathrm{rpm}$ orbital speed.

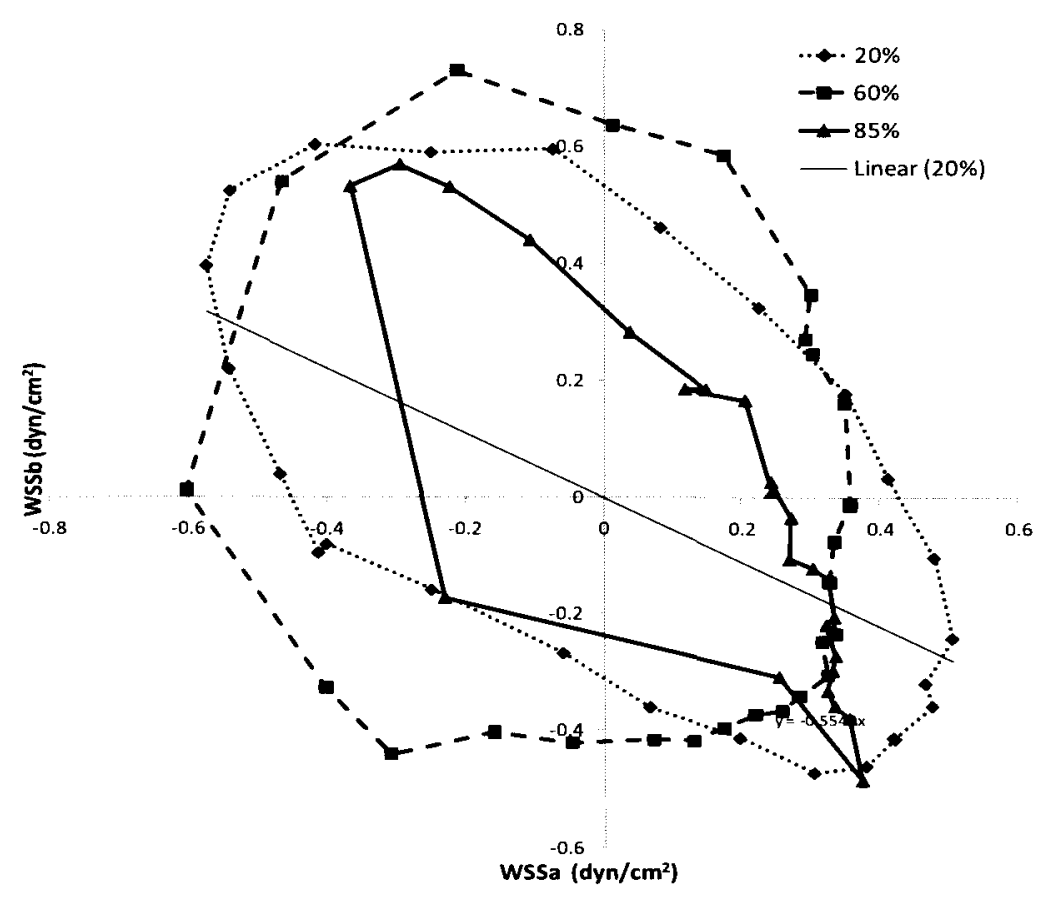

Figure 4.5. An example plot of $1^{\text {st }}$ principal axis WSS vs. $2^{\text {nd }}$ principal axis WSS for different radial locations $(20 \%, 60 \%$, and $85 \%)$ at $50 \mathrm{rpm}$ orbital speed. 
Table 4.2. DOSI at different orbital speeds and radial locations.

\begin{tabular}{ccccc}
\hline Orbital speed (rpm) & Radial location (\%) & OSI $_{1}$ & OSI $_{2}$ & DOSI \\
\hline \multirow{2}{*}{50} & 20 & 0.984 & 0.949 & 0.036 \\
& 60 & 0.862 & 0.847 & 0.018 \\
& 85 & 0.724 & 0.691 & 0.047 \\
\hline \multirow{2}{*}{100} & 20 & 0.892 & 0.620 & 0.305 \\
& 60 & 0.917 & 0.075 & 0.918 \\
& 85 & 0.986 & 0.006 & 0.994 \\
\hline \multirow{2}{*}{150} & 20 & 0.839 & 0.352 & 0.581 \\
& 60 & 0.994 & 0.111 & 0.888 \\
\hline
\end{tabular}

A.

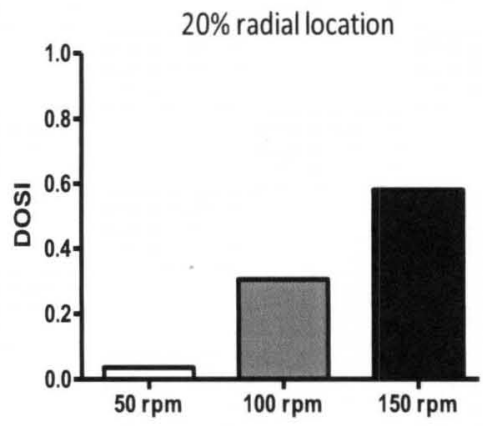

B.

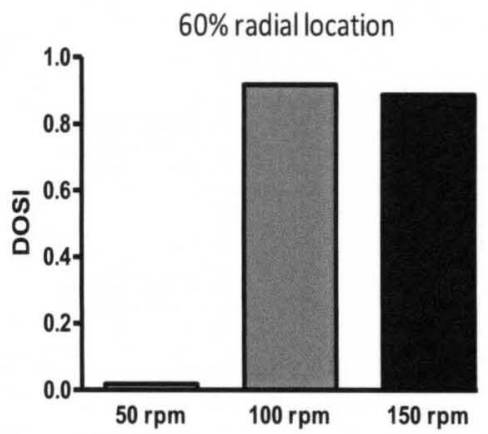

C.

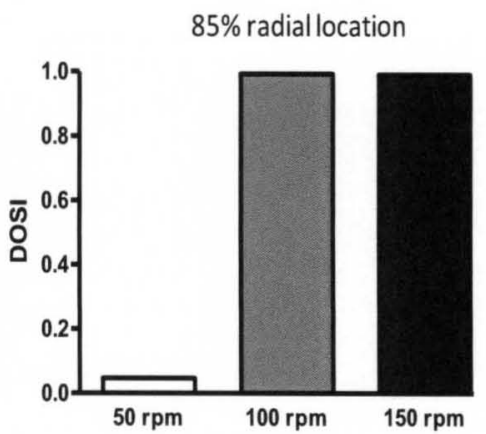

Figure 4.6. Directional oscillatory shear index (DOSI) at different radial locations and orbital speeds. Figure 4.6A, 4.6B and 4.6C indicate different DOSI values at 20\%, 60\% and $85 \%$ radial locations at different orbital speeds. 


\subsubsection{Effects of oscillatory shear on cell proliferation and area and cell shape index}

Representative images of cells with and without shear exposure at different radii at $100 \mathrm{rpm}$ orbital speed are shown in Figure 4.7A. The cells were elongated and less proliferated near the wall (at $85 \%$ radial location), while there was a substantial increase in proliferation near the center of the dish (at $20 \%$ radial location) compared to cells grown without shear exposure. The differences in cell proliferation and morphology under shear stress may be due to either cell nuclei division or cell stretching by fluidic stresses. Percent HUVEC proliferation, cell area and cell shape index versus DOSI, shear magnitudes, and orbital speeds at three radial locations are shown in Figure 4.7B. Cell proliferation did not change significantly with radial locations at orbital speed of $50 \mathrm{rpm}$ (Figure 4.7B). At orbital speed of $100 \mathrm{rpm}$, cell proliferation was significantly higher near the center ( $20 \%$ radial location) and lower near the edge of the dish $(85 \%$ radial location) compared to unstressed cells. At $150 \mathrm{rpm}$, cell proliferation was significantly higher at $20 \%$ and $60 \%$ radial locations, but lower at $85 \%$ radial location, than unstressed cells. Cell area was significantly larger for all orbital speeds and at all radial locations than for unstressed cells, and consistently increased with increasing shear at $100 \mathrm{rpm}$ and $150 \mathrm{rpm}$. Shape index was significantly lower than unstressed controls at all locations and for all speeds (Figure 4.7B). Shape index decreased with increasing radial location, particularly at the two higher speeds, for which DOSI was high near the wall of the dish. 
A

No shear

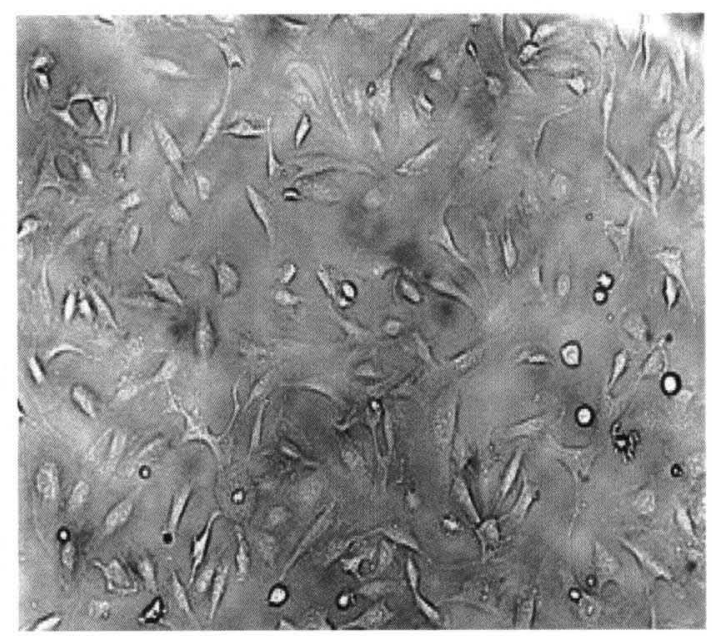

$20 \%$ radial location of the dish

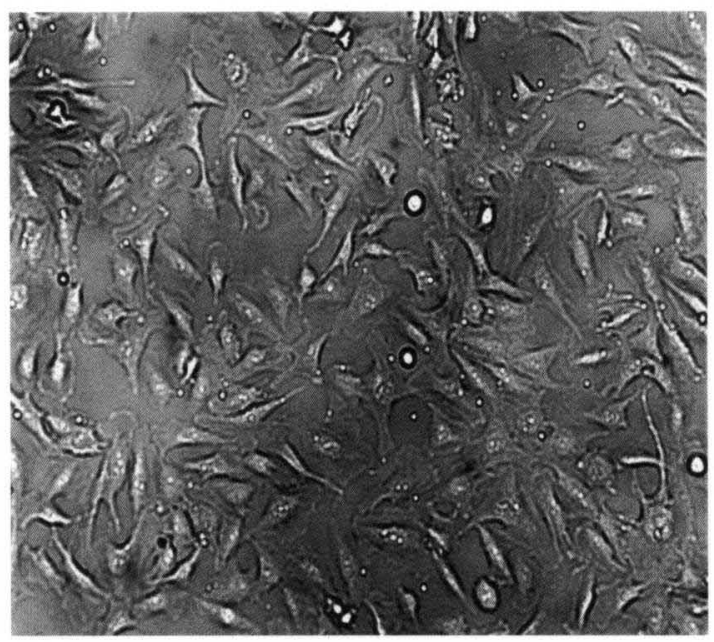

$85 \%$ radial location of the dish

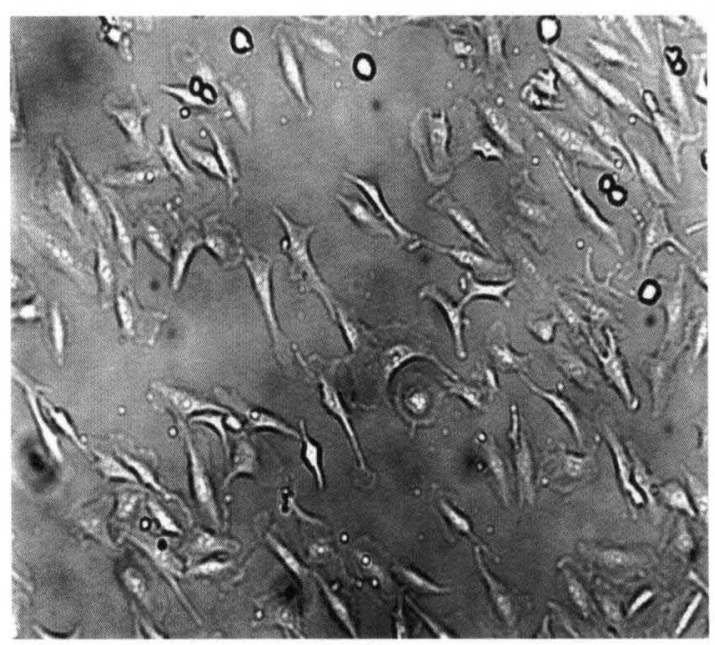


B
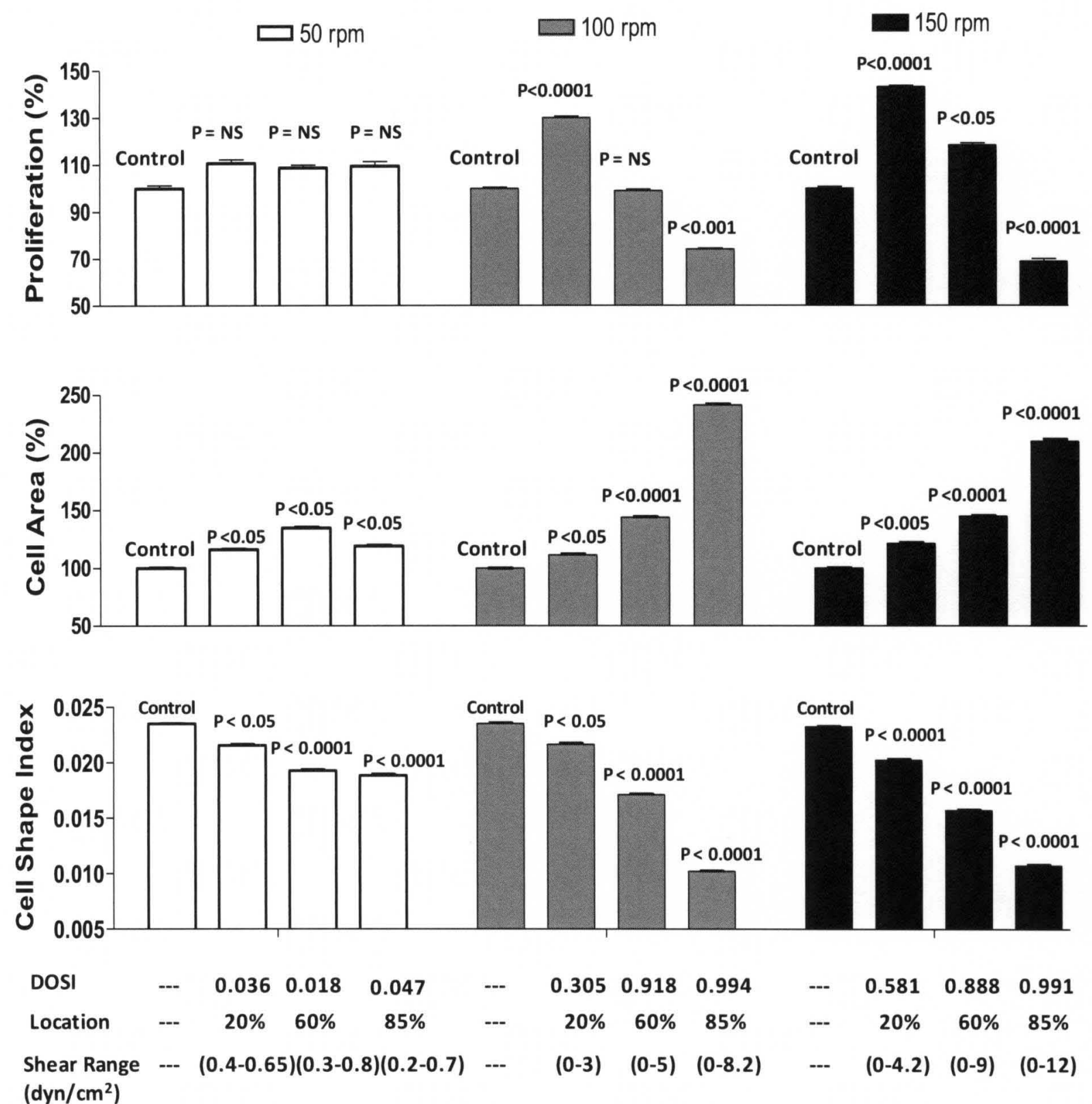

Figure 4.7. Effects of DOSI and shear magnitude on cell proliferation, area and shape

index at three orbital speeds and at three radial locations. Figure 4.7A compares example cell images unexposed and exposed to shear levels at two different radial locations at orbital speed of $100 \mathrm{rpm}$. Figure 4.7B indicates cell proliferation, area and shape index versus DOSI and shear magnitude at three radial locations at three orbital speeds. 


\subsubsection{Individual and interacting effects of DOSI, shear magnitude and orbital speed on cell proliferation}

Poisson statistical analyses of individual and interacting effects of DOSI, shear magnitude, and orbital speeds on cell proliferation are shown in Table 4.3. Of the singlefactor models, DOSI provided the smallest deviance of 1828 . Adding orbital speed along with DOSI in a two-factor additive model $(\mathrm{D}+\mathrm{S})$ reduced deviance significantly, but shear magnitude and speed $(\mathrm{M}+\mathrm{S})$ reduced deviance the most to 1318. Of the two-factor interacting models (DM, DS and MS), only DM reduced the deviance significantly. The three-factor additive model $\mathrm{D}+\mathrm{M}+\mathrm{S}$ reduced deviance to 1253 , a small decrease compared to the two-factor model $\mathrm{M}+\mathrm{S}$. The three-factor model with single interaction S+DM provided another small reduction of deviance to 1215 , while the fully interacting model DM+DS+MS yielded the smallest deviance of 1198 .

Table 4.3B showed the parameter estimates and significance (p-values) for the three-factor additive and interacting models. All individual factors and their interactions had statistically significant effects in these models.

\subsubsection{Individual and interacting effects of DOSI, shear magnitude and orbital speeds on cell area}

Compared to the null model, which had a deviance of 128.1, DOSI reduced deviance to 79.5, the lowest among all one-factor models of cell area (Table 4.3A). Among the two-factor models, the additive $\mathrm{D}+\mathrm{M}$ provided the lowest deviance of 76.2 . The three-factor model with two interactions $\mathrm{DM}+\mathrm{MS}$ was as good as the fully interactive DM+MS+DS, with deviances of 71.6. The lack of significance of the DS 
interaction was confirmed in Table 4.3B, where DS is the only individual or interacting effect on cell area that was not significant.

\subsubsection{Individual and interacting effects of DOSI, shear magnitude and orbital speeds on cell shape index}

The one-factor model D provided deviance of 0.0220 , which was as low as any other models, other than the two-factor model D+M (deviance of 0.0210 ) and the threefactor models $\mathrm{D}+\mathrm{M}+\mathrm{S}$ (deviance of 0.0210 ) and $\mathrm{S}+\mathrm{DM}$ (deviance of 0.0210 ) (Table 4.3A). The individual effects of DOSI and shear magnitude were statistically significant, but that of orbital speed was not (Table 4.3B). The interactions DM and DS were significant, but MS was not. 
Table 4.3A. Deviances of Poisson log-linear model of cell proliferation, gamma distribution of cell area and Gaussian distribution for cell shape index responses to DOSI, shear magnitude and orbital speed.

\begin{tabular}{|c|c|c|c|c|c|c|}
\hline \multirow[b]{2}{*}{ Models } & \multicolumn{2}{|c|}{ Effects on cell proliferation } & \multicolumn{2}{|c|}{ Effects on cell area } & \multicolumn{2}{|c|}{ Effects on cell shape index } \\
\hline & Deviance & $\begin{array}{l}\text { Degrees of } \\
\text { Freedom }\end{array}$ & Deviance & $\begin{array}{l}\text { Degrees of } \\
\text { Freedom }\end{array}$ & Deviance & $\begin{array}{c}\text { Degrees of } \\
\text { Freedom }\end{array}$ \\
\hline Null & 2390 & 251 & 128.1 & 696 & 0.0340 & 722 \\
\hline \multicolumn{7}{|l|}{$\begin{array}{c}\text { One-factor } \\
\text { models }\end{array}$} \\
\hline DOSI(D) & 1828 & 250 & 79.5 & 695 & 0.0220 & 721 \\
\hline $\begin{array}{c}\text { Shear } \\
\text { magnitude(M) }\end{array}$ & 1974 & 250 & 86.9 & 695 & 0.0230 & 721 \\
\hline $\begin{array}{l}\text { Orbital speed } \\
\text { (S) }\end{array}$ & 2380 & 250 & 111.7 & 695 & 0.0271 & 721 \\
\hline \multicolumn{7}{|l|}{$\begin{array}{c}\text { Two-factor } \\
\text { models }\end{array}$} \\
\hline $\mathrm{D}+\mathrm{M}$ & 1811 & 249 & 76.2 & 694 & 0.0210 & 720 \\
\hline$D+S$ & 1582 & 249 & 79.3 & 694 & 0.0220 & 720 \\
\hline $\mathrm{M}+\mathrm{S}$ & 1318 & 249 & 86.0 & 694 & 0.0230 & 720 \\
\hline DM & 1631 & 250 & 81.2 & 695 & 0.0220 & 721 \\
\hline DS & 1901 & 250 & 87.5 & 695 & 0.0230 & 721 \\
\hline MS & 2080 & 250 & 100.8 & 695 & 0.0250 & 721 \\
\hline \multicolumn{7}{|l|}{$\begin{array}{l}\text { Three-factor } \\
\text { models }\end{array}$} \\
\hline $\mathrm{D}+\mathrm{M}+\mathrm{S}$ & 1253 & 248 & 75.4 & 693 & 0.0210 & 719 \\
\hline $\mathrm{D}+\mathrm{MS}$ & 1820 & 249 & 78.5 & 694 & 0.0220 & 720 \\
\hline $\mathrm{M}+\mathrm{DS}$ & 1895 & 249 & 84.6 & 694 & 0.0220 & 720 \\
\hline $\mathrm{S}+\mathrm{MD}$ & 1215 & 249 & 81.2 & 694 & 0.0210 & 720 \\
\hline $\mathrm{DS}+\mathrm{MS}$ & 1899 & 249 & 87.3 & 694 & 0.0230 & 720 \\
\hline $\mathrm{DM}+\mathrm{DS}$ & 1512 & 249 & 81.1 & 694 & 0.0220 & 720 \\
\hline $\mathrm{DM}+\mathrm{MS}$ & 1340 & 249 & 71.6 & 694 & 0.0230 & 720 \\
\hline $\mathrm{DM}+\mathrm{MS}+\mathrm{DS}$ & 1198 & 248 & 71.6 & 694 & 0.0220 & 719 \\
\hline
\end{tabular}


Table 4.3B. Individual and interacting effects of DOSI, shear magnitude and orbital speed on cell proliferation, cell area and cell shape index.

\begin{tabular}{|c|c|c|c|c|c|c|}
\hline \multirow[b]{2}{*}{ Parameters } & \multicolumn{2}{|c|}{ Effects on cell proliferation } & \multicolumn{2}{|c|}{ Effects on cell area } & \multicolumn{2}{|c|}{ Effects on cell shape index } \\
\hline & $\begin{array}{c}\text { Regression } \\
\text { coefficient } \\
\beta \\
\end{array}$ & $\begin{array}{c}\text { Significance } \\
\text { P } \\
\end{array}$ & $\begin{array}{c}\text { Regression } \\
\text { coefficient } \\
\beta \\
\end{array}$ & $\begin{array}{c}\text { Significance } \\
\text { P } \\
\end{array}$ & $\begin{array}{c}\text { Regression } \\
\text { coefficient } \\
\beta \\
\end{array}$ & $\begin{array}{c}\text { Significance } \\
\mathrm{P} \\
\end{array}$ \\
\hline \multicolumn{7}{|c|}{$\begin{array}{l}\text { Individual effects from three-factor additive model } \\
\qquad(D+M+S)\end{array}$} \\
\hline DOSI(D) & -0.179 & $<0.001$ & -0.00319 & $<0.001$ & -0.00559 & $<0.001$ \\
\hline $\begin{array}{c}\text { Shear } \\
\text { magnitude(M) }\end{array}$ & -0.0990 & $<0.001$ & -0.000399 & $<0.001$ & -0.000760 & $<0.001$ \\
\hline Orbital speed(S) & 0.00427 & $<0.001$ & 7.97E-06 & $<0.01$ & $-1.050 \mathrm{E}-05$ & $\begin{array}{c}\text { Not } \\
\text { significant }\end{array}$ \\
\hline \multicolumn{7}{|c|}{ Interacting effects from three-factor interactive model (DM+DS+MS) } \\
\hline DM & -0.254 & $<0.001$ & -0.00136 & $<0.001$ & -0.00167 & $<0.001$ \\
\hline DS & 0.000840 & $<0.001$ & $-2.68 \mathrm{E}-06$ & $\begin{array}{c}\text { Not } \\
\text { significant }\end{array}$ & $-2.28 \mathrm{E}-05$ & $<0.05$ \\
\hline MS & 0.000400 & $<0.001$ & $5.55 \mathrm{E}-06$ & $<0.001$ & $3.00 \mathrm{E}-06$ & $\begin{array}{c}\text { Not } \\
\text { significant }\end{array}$ \\
\hline
\end{tabular}

\subsubsection{Effects of DOSI, shear magnitude, and orbital speeds on cell orientation}

Cell orientation was random near the center of the dish for all speeds, as indicated by the large error bars in Figure 4.8A at radial location of $20 \%$. Orientation was random at all radial locations for orbital speed of $50 \mathrm{rpm}$, but alignment with the dominant tangential shear direction increased with radial location at the higher two speeds. Figure 4.8B, C, and D, further supported the previous statement. From those figures, it is easily observed that cells are aligned towards the dominant shear direction with increasing 
radial locations from the center to the side walls at higher orbital speeds of 100 and 150 rpm.

The individual and interacting effects of DOSI, magnitude, and orbital speeds on cell orientation with the circular regression model are shown in Table 4.4. Higher loglikelihood indicated better fit of the model to the actual probability distribution of the angle of cell orientation. DOSI provided the highest log-likelihood of 5.26 among onefactor models, while $\mathrm{D}+\mathrm{S}$ was best among two-factor models with a log-likelihood of 7.26. All factors had statistically significant individual and interacting effects except for the individual effect of shear magnitude in the three-factor additive model.

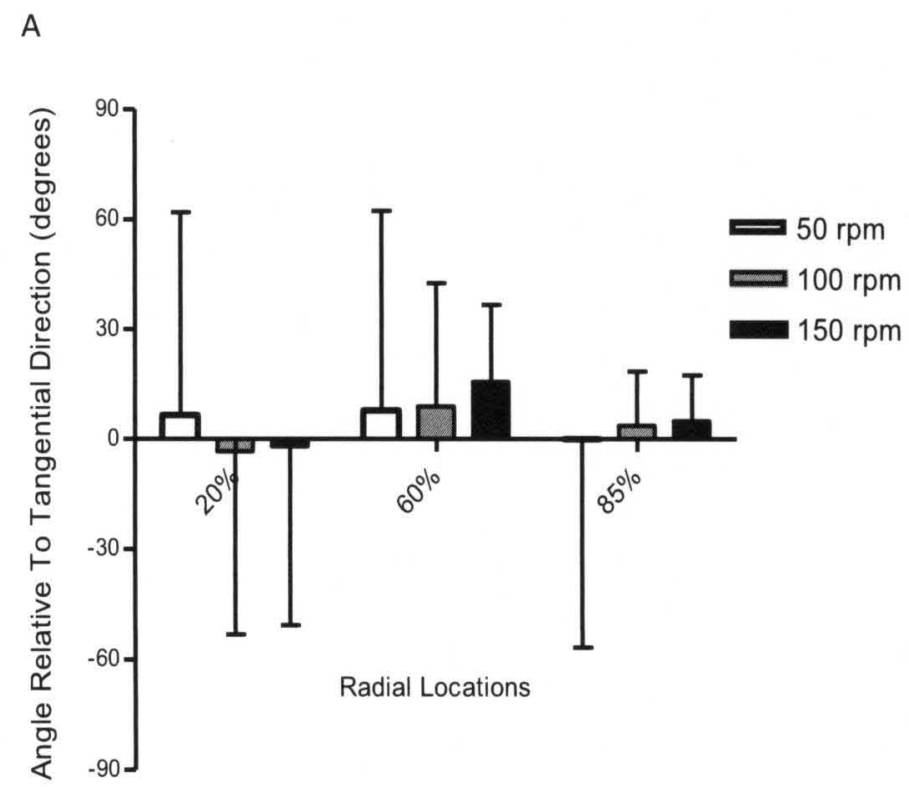


B

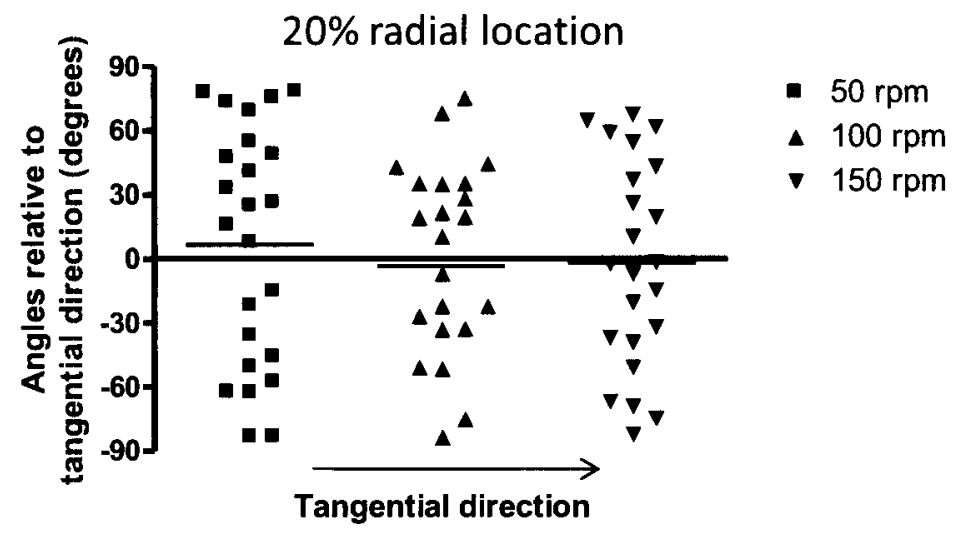

C

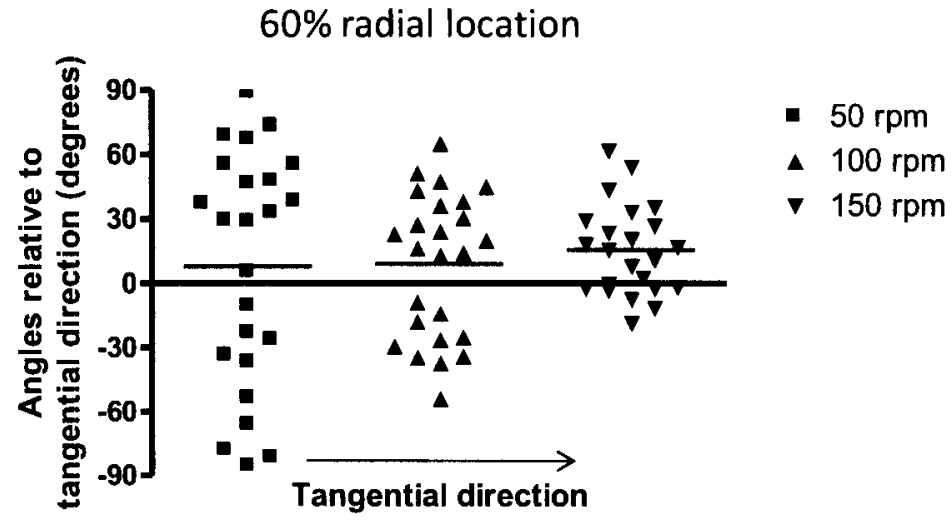

$\mathrm{D}$

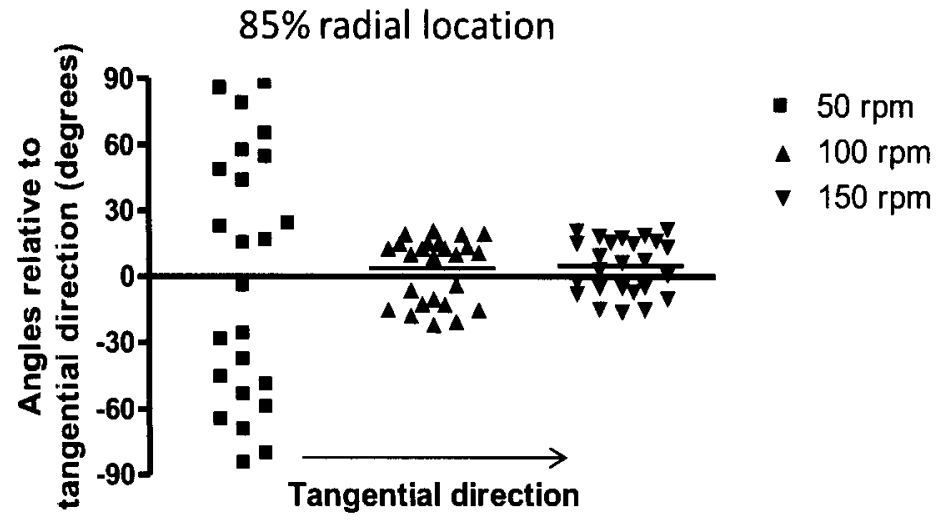

Figure 4.8. Angle of cell orientation relative to tangential flow direction at different orbital speeds and at different radial locations. 
Table 4.4A. Log likelihood of circular regression models of cell orientation to DOSI, shear magnitude and orbital speed.

\begin{tabular}{cc}
\hline & $\begin{array}{c}\text { Effects on angle of cell orientation } \\
\text { Models }\end{array}$ \\
& Log-likelihood \\
\hline Null & 3.80 \\
& \\
\hline One-Factor models & 5.26 \\
DOSI(D) & 4.62 \\
Shear magnitude(M) & 4.48 \\
Orbital Speed(S) & \\
& 5.45 \\
Two-factor models & 7.26 \\
D+M & 7.15 \\
D+S & 5.03 \\
M+S & 4.62 \\
DM & 4.93 \\
DS & \\
MS & 7.56 \\
& 5.45 \\
Three-factor models & 4.53 \\
D+M+S & 7.30 \\
D+MS & 5.19 \\
M+DS & 6.26 \\
S+MD & 4.35 \\
DM+DS & 6.40 \\
DM+MS & \\
DS+MS & \\
DM+MS+DS & \\
\hline & \\
& \\
\hline
\end{tabular}

Table 4.4B. Individual and interacting effects of DOSI, shear magnitude and orbital speed on cell orientation by circular regression model.

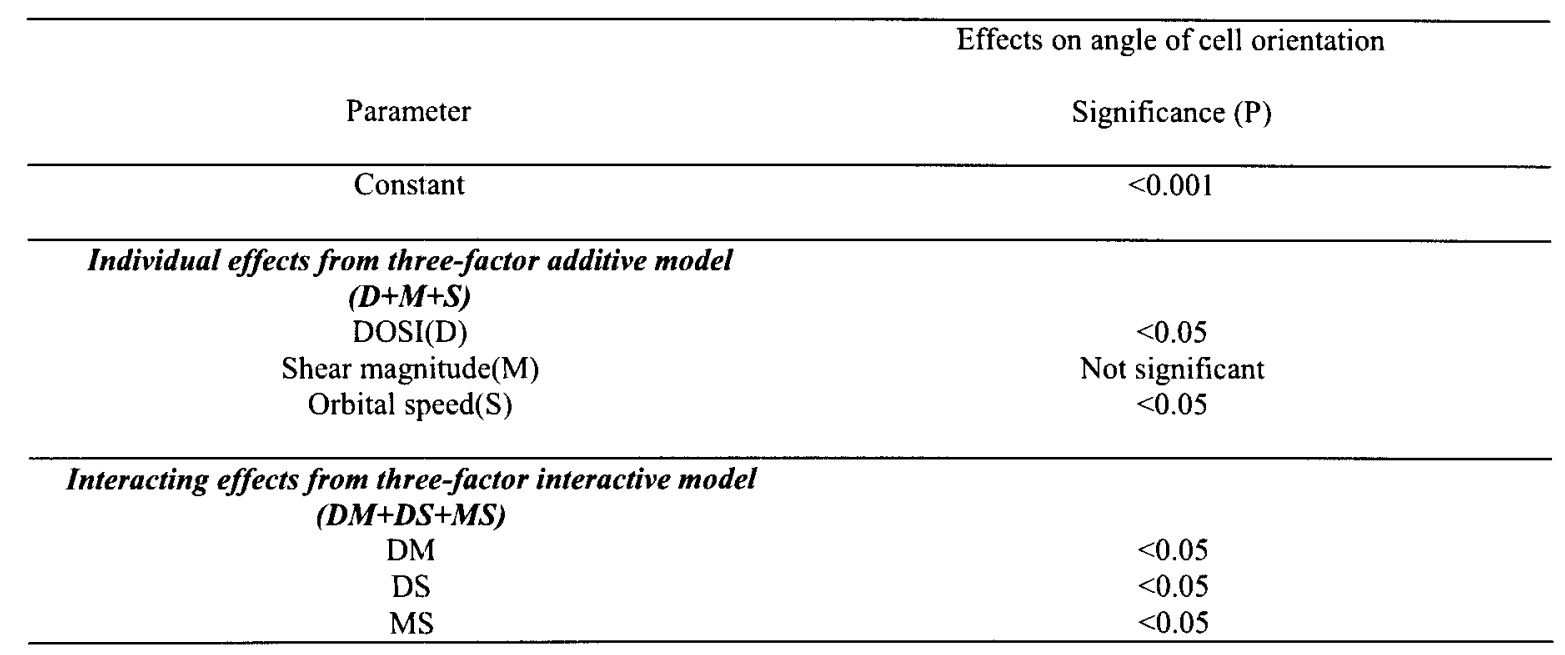




\subsubsection{CONCLUSION}

Orbital shaker was used to generate shear on endothelial cells and CFD was applied to quantify the exerted shear. The usefulness of the orbital shaker to generate shear on cells has been described by previous authors (32-36). One of the major advantages of using orbital shaker is that it can be used to study multiple cell responses since many culture dishes can stacked. Further, orbital shear model can be physiologically relevant which can describe the complex, disturbed, and orbital blood flow more accurately after angioplasty compared to laminar blood flow (113). Computational results for WSS varied substantially with radial locations within the dish. Nearly uniform shear fields of low magnitude were observed near the center for all orbital speeds tested, whereas high and fluctuating shear ranges were observed near the vertical wall of the dish. A new hemodynamic parameter, directional oscillatory shear index (DOSI), was developed to quantify the directionality of the two-dimensional shear found in the dish. DOSI approached zero near the center of the dish, indicating prevalence of bidirectional (radial and tangential) shear, whereas DOSI approached unity near the vertical wall of the dish, indicating unidirectional tangential WSS. These computed WSS and DOSI values were compared to experimental observations of cell responses. It was observed that cell proliferation was not significantly impacted by low WSS up to about $1 \mathrm{dyn} / \mathrm{cm}^{2}$, but was greater under bidirectional oscillatory shear of 3-4 $\mathrm{dyn} / \mathrm{cm}^{2}$. Cell proliferation was significantly decreased for unidirectional WSS of 8-12 $\mathrm{dyn} / \mathrm{cm}^{2}$. The cell areas also were significantly larger than controls for all shear conditions, but were especially large under unidirectional tangential WSS near the wall of the dish. Cell shape index decreased with increasing shear magnitude and DOSI, and 
cells aligned with the tangential direction of the flow near the wall of the dish. Different statistical methods were introduced thereafter to understand the individual and interacting effects of those simultaneous affecting factors (DOSI, shear magnitude and orbital speeds) on different cellular responses. Those multi-factorial statistical analyses found that DOSI was the strongest determinant of proliferation, area, shape index, and angle of cell orientation among one factor models. If the effects of DOSI predominate, these results indicate that more, smaller, less elongated and randomly oriented cells grow in bidirectional shear with nearly equal oscillatory fluctuations (low DOSI), and fewer, larger cells with greater elongation and more uniform orientation are promoted in unidirectional oseillatory shear (high DOSI). While the impacts of steady and oscillatory shear on endothelial cell responses have been previously observed, these results demonstrate that the directionality of shear also significantly affects cellular responses. These results might have implications for models dealing with atherosclerotic vascular lesion development in human arteries. For example, it is known that there is an increased propensity for atherosclerotic lesion development at arterial branch points (65), where there is a change in the direction of the flow. Further, pulsatility, which occurs throughout most of the arterial system, has been shown to influence cell responses. A study (25) found that unidirectional pulsatile shear stress on cultured human aortic endothelial cells was a stronger stimulus than steady shear for inducing cell orientation. In another effort, (94) the pulsatility was found to increase significantly the rates at which endothelial cells elongated and realigned, compared to steady flow. Since the flow is pulsatile, therefore one could model changes in oscillatory shear described in this study to understand the mechanisms involved in increased lesion development at these areas. Also 
previous study (88) indicated that the effects of unidirectional oscillatory shear stress on adhesion molecule expression in cultured human endothelium. The study revealed that unidirectional oscillatory shear stress can stimulate mononuclear leukocyte adhesion and migration into the arterial wall. Therefore, determination of location specific changes (such as adhesion molecule expression) in endothelial cells cultured in orbital shakers might provide clues how multi-directional oscillatory shear controls vascular lesion development. 
CHAPTER FIVE

\author{
EFFECTS OF DOSI AND SHEAR MAGNITUDES OF OSCILLATING SHEAR \\ ON CELL PROLIFERATION, MORPHOLOGY, AND ATHEROGENIC GENE \\ EXPRESSIONS AT THE mRNA LEVEL IN THE PRESENCE OF LTB AND LPS $_{4}$
}

\title{
5.1. INTRODUCTION AND BACKGROUND
}

\subsubsection{Mechanism of endothelial cellular responses to fluidic shear and effects of hemodynamic parameters on vascular inflammation and atherosclerosis}

Since hemodynamic parameters especially fluidic shear stress have been shown to play an important role in regulating various cellular structures and responses, it is therefore important to understand the mechanism of endothelial cellular responses under the exposure of fluid stress. Previous investigations have documented various effects of shear stress, both steady and pulsatile, on cellular responses that eventually significantly alters the various intracellular molecules. More importantly, alteration of different intracellular pathways and gene expressions under exposure of fluid stress were shown to have serious impacts on the disease formation like atherosclerotic plaque development. 


\subsubsection{Effects of fluid shear stress on various intracellular signaling molecules and gene expressions}

Malek, et al. (114) investigated that endothelial cell exposed to fluid shear stress (FSS) undergoes cell shape change, alignment and microfilament network remodeling in the direction of flow by an unknown mechanism. They concluded that FSS has a profound effect on endothelial cell shape and F-actin network by a mechanism which depends on TK activity, intracellular calcium, and an intact microtubule network, but is independent of protein kinase $\mathrm{C}$, intermediate filaments and shear and stretch activated mechanosensitive channels. Nollert, et al. (14) studied the mechanism by which endothelial cells detect the presence of mechanical stress and can convert this force into intracellular signals. They have studied cultured endothelial cells which line the blood vessels and form the interface between the blood and the vessel wall. Their results demonstrated that endothelial cells respond in specific ways to the initiation of flow, and mass transfer and fluid shear stresses both play a role in modulation of intracellular signal transduction and metabolism. Chien, et al. (115) studied the effects of shear stress on endothelial cells in modulating endothelial functions, including the regulation of genes encoding for vasoactive substances, growth factors, adhesion molecules, chemotactic agents, coagulation factors, and photo-oncogenes. In their research, it has been demonstrated that shear stress (about 16 dynes $/ \mathrm{cm}^{2}$ ) induced a transient activation of the monocyte chemotactic protein-1 (MCP-1) gene and the tissue factor (TF) gene in cultured human umbilical vein endothelial cells (HUVEC). In another effort, Vara, et al. (116) investigated the gene expression of human endothelial cells seeded on cylindrical viscoelastic conduits ( $5 \mathrm{~mm}$ diameter) under shear stress of value about 1.4 Pa. After four 
hour of recovery period, there were no significant differences in gene intensity, suggesting that this change was transient. These data in this experiment proved that $\mathrm{m}$ RNA can be obtained from EC's seeded on tubular conduits, exposed to shear stress. Lin, et al. (117) further investigated the effects of shear stress on tissue factor gene expression in endothelial cells. Kondapalli, et al. (5) assessed the differential gene expression of p120 Catenin, Kaiso and VE-cadherin in human coronary artery endothelial cells (HCAEC) at specific locations along the wound border to further the understanding of molecular mechanisms involved in wound closure. DePaola, et al. (118) studied the effect of shear stress (ranging from 10 to $35 \mathrm{dyne} / \mathrm{cm}^{2}$ ) gradients on endothelial cell proliferation and monolayer integrity. In his study, bovine aortic endothelial cells grown to confluence on glass cover slips were exposed to fluid forces using a cone and plate or parallel plate flow chamber. As a result, endothelial monolayers exposed to steady disturbed flows for 48 hours exhibited increased DNA synthesis in areas with a high shear stress gradient and an altered cell density distribution that clearly showed cell migration in the downstream direction. Hsiai, et al. (27) developed micro electrical mechanical systems (MEMS) sensor, comparable to the size of an endothelial cell $(2 \times 80$ $\mu \mathrm{m})$, to deliver the spatial and temporal resolution necessary at a frequency response of greater than $100 \mathrm{~Hz}$. They provided the first evidence of real time wall shear stress on EC coupled with real time gene expression of monocyte chemoattractant protein (MCP-1). Guild, et al. (119) studied hemodynamic (laminar shear stress) alteration of monocyte recruitment and adhesion in model blood vessel systems, focusing on the hemodynamic effects (low shear of around 2 dyne $/ \mathrm{cm}^{2}$ and high shear of about $15 \mathrm{dynes} / \mathrm{cm}^{2}$ ) of recruitment factors like monocyte chemotactic protein one (MCP-1), gene expression in 
human aortic endothelial cell (HAEC). This result implied that the transient mRNA increases appears to be an in vitro response to the onset of flow from static conditions and the cellular pathways responsible for the high shear transient interact with long term low shear preconditioning. Chachisvilis, et al. (120) et al. found that G protein -coupled receptors can sense fluid shear stress in endothelial cells. They suggest that GPCRs are involved in mediating primary mechanochemical signal transduction in endothelial cells.

\subsubsection{Inflammation and atherosclerosis}

Atherosclerosis is one of the major causes of heart attack and stroke and it has the highest mortality in western countries. It is a condition in which fatty materials collects along the walls of arteries. This fatty material thickens, hardens (by formation of calcium deposits), and eventually blocks the arteries. The atherosclerotic plaque formed in arteries is developed by accumulation of fat, cholesterol, calcium and other substances which are naturally found in blood. Over the period of time, the plaque gets hard and narrows the arteries. This will limit the flow of oxygen-rich blood to organs and other parts of the body which eventually causes heart attack or stroke. Figure 5.1 shows plaque formation. The fatty materials deposit on the wall of the arteries resulting from a tear in the artery, and eventually blocks the artery (Figure 5.1A). Figure 5.1B shows closer view in a cut section of a torn artery. 
A.
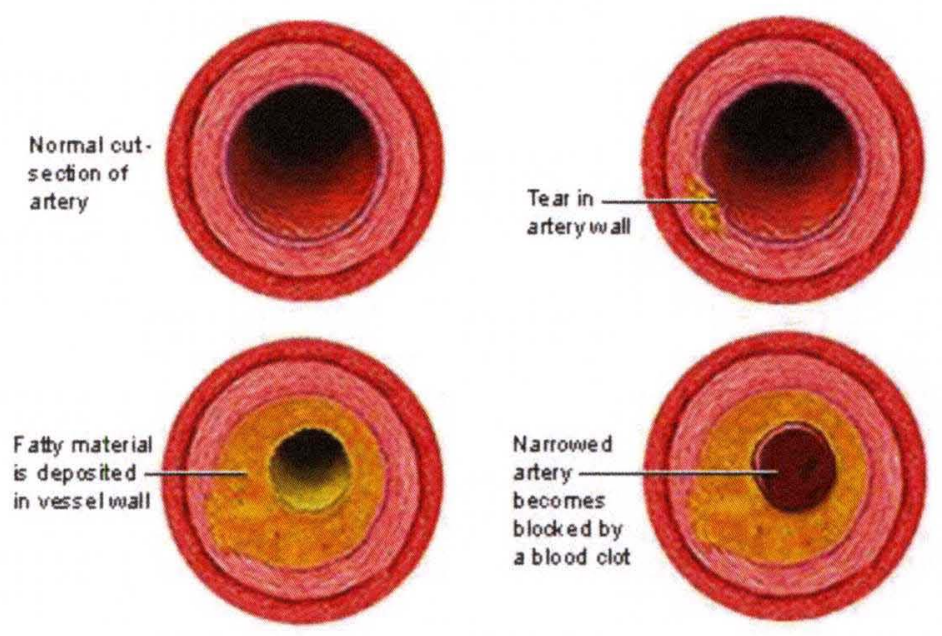

ADAM.

B.

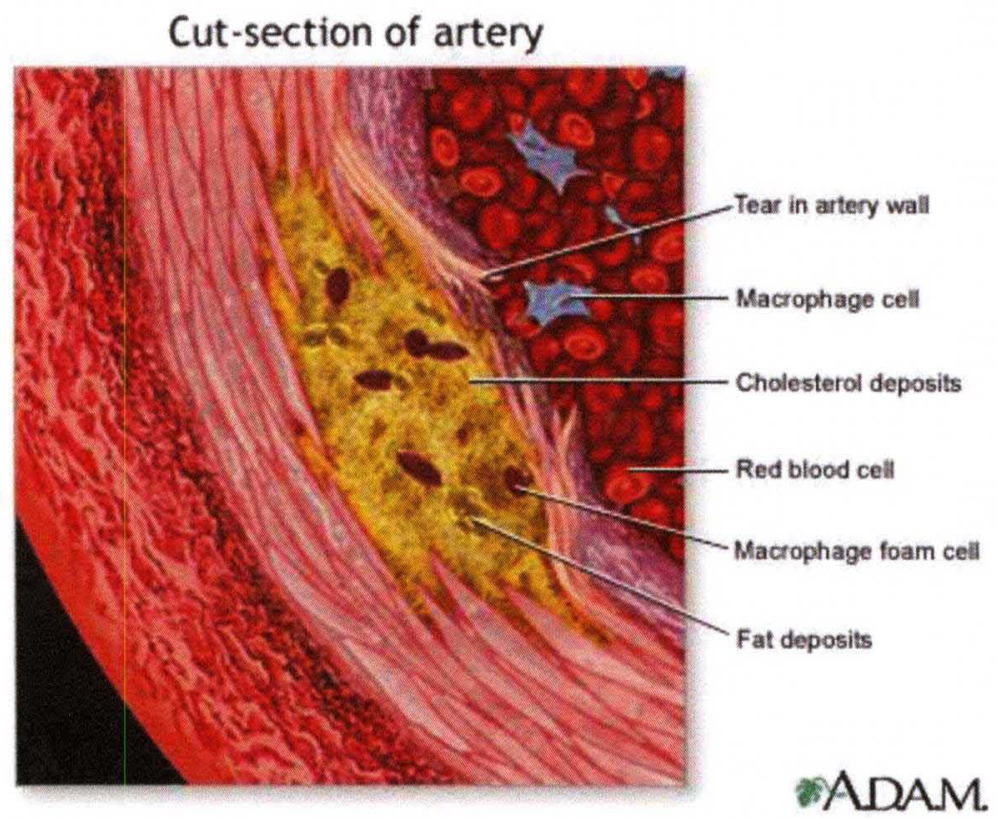

Figure 5.1. Formation of atherosclerosis plaque in a human artery. Figure 5.1A indicates the steps for atherosclerotic plaque formation, and Figure 5.1B shows a cut-section of artery (http://adam.about.net/encyclopedia/infectiousdiseases/Atherosclerosis.htm) 
Over the years, researchers investigated the cause of formation of atherosclerotic plaque and two main factors, namely inflammation and blood flow/shear flow, have generated considerable interest in having their significant roles in formation of the plaque $(121,122)$. Inflammation is a part of a complex biological responses by vascular tissues to harmful stimuli like pathogens, damaged cells, or irritants (123). It is characterized by increased blood flow to tissue, increased temperature, redness, swelling, and pain. In the review of Libby, et al. (121), the data indicated that the link between inflammation and atherosclerosis can yield predictive and prognostic information of considerable clinical utility. Further, the effects of blood flow in atherosclerosis are based on the observation that vascular inflammation and atherosclerosis plaque is distributed near the side of branches, where blood flow is non uniform. Since blood flow exerts shear stress on the vessel wall causing the cell physiological alteration, it has a serious impact on atherosclerotic plaque formation as the nature of shear stresses are different (steady unidirectional or oscillatory) depending on the flow through different regions of arches and braches of an arterial tree (124). Shear stress exerted by blood flow, therefore has a key role in the formation of atherosclerotic plaque, and it is desirable to understand the effects of different shear on atherosclerosis.

\subsubsection{The role of fluid shear stress on atherosclerosis}

As discussed previously, fluidic shear is considered an important parameter nowadays which affects the atherosclerotic plaque formation. The development of atherosclerosis is initiated by a dysfunctional state of the vascular endothelium caused by fluid wall shear stress, resulting from the flow of blood through the artery $(121,122$, 
125). Ridger, et al. (124) reviewed the effects of blood flow and shear stress on the leukocyte adhesion cascade and endothelial cell function. According to this study, high unidirectional laminar shear stress induces pronounced anti-inflammatory effects on cultured EC, whereas low, oscillatory shear has been shown to potentiate proinflammatory activation of EC and may be a positive regulator of vascular inflammation at atheroprone sites. Traub, et al. (126) further suggested that steady laminar shear stress stimulates cellular responses that are essential for endothelial cell function and are atheroprotective. Binding and recruitment of those circulating leukocytes to the vascular endothelium are key steps in atherosclerotic lesion development and are mediated through diverse cellular adhesion molecules that are expressed on the surface of vascular endothelial cells. Of those, intracellular adhesion molecule-1 (I-CAM1), endothelialleukocyte adhesion molecule-1 (E-Selectin), and interleukin-6 (IL-6) play important roles in atherosclerotic lesion development (127-132). It is also well known that endothelial intercellular adhesion molecule-1 (ICAM-1), vascular cell adhesion molecule-1 (VCAM1), and E-Selectin play a critical role in the development of atherosclerosis by sequestering the circulating leukocytes $(133,134)$. These molecules mediate these actions by supporting adhesive interactions and are involved in cell signaling (135, 136). Interleukin-6 (IL-6), a pro inflammatory cytokine and its signaling events have shown contribution to atherosclerotic plaque development and plaque destabilization (137). While investigating the cause of formation of atherosclerotic plaque, previous studies found several genes affecting plaque formation that are altered by fluidic shear stress. Further, previous studies indicated that shear stress affects the function of endothelial cells, leading to both faster and slower tissue responses. Transmission of the shear stress 
signal throughout the vascular cell involves cytoskeletal and biochemical elements and influences gene expression, including I-CAM1, E-Selectin and IL-6 (138-140).

\subsubsection{Role of Leukotrienes and its receptors in atherosclerosis}

Although inflammatory reactions have a proven role in atherosclerotic plaque formation, the importance of leukotrienes in inflammation was well recognized in recent studies. Leukotrienes are inflammatory mediators derived from the 5-lipoxygenase pathways of arachidonic metabolism (141). Medications targeting the leukotriene pathway were introduced in the treatment of disease such as asthma $(142,143)$. Further, a recent study indicates that the leukotriene pathway represents a common link between asthma and atherosclerosis (144). According to Back, et al. (145), the key role in both diseases is played by the enzyme 5-lipoxygenase (5-LO) and 5-LO-activating protein (FLAP) which catalyze the metabolism of endogenous arachidonic acid, leading to the formation of the unstable precursor leukotriene $\mathrm{A}_{4}$. Further, it was found that a slowreacting substance of anaphylaxis is composed of cysteinyl leukotrienes $\mathrm{C}_{4}\left(\mathrm{LTC}_{4}\right)$, Leukotriene $\mathrm{D}_{4}\left(\mathrm{LTD}_{4}\right)$ and Leukotriene $\mathrm{E}_{4}\left(\mathrm{LTE}_{4}\right)(146)$. The pathways for leukotriene biosynthesis were described by Mathis, et al. (147) which are shown in Figure 5.2. 


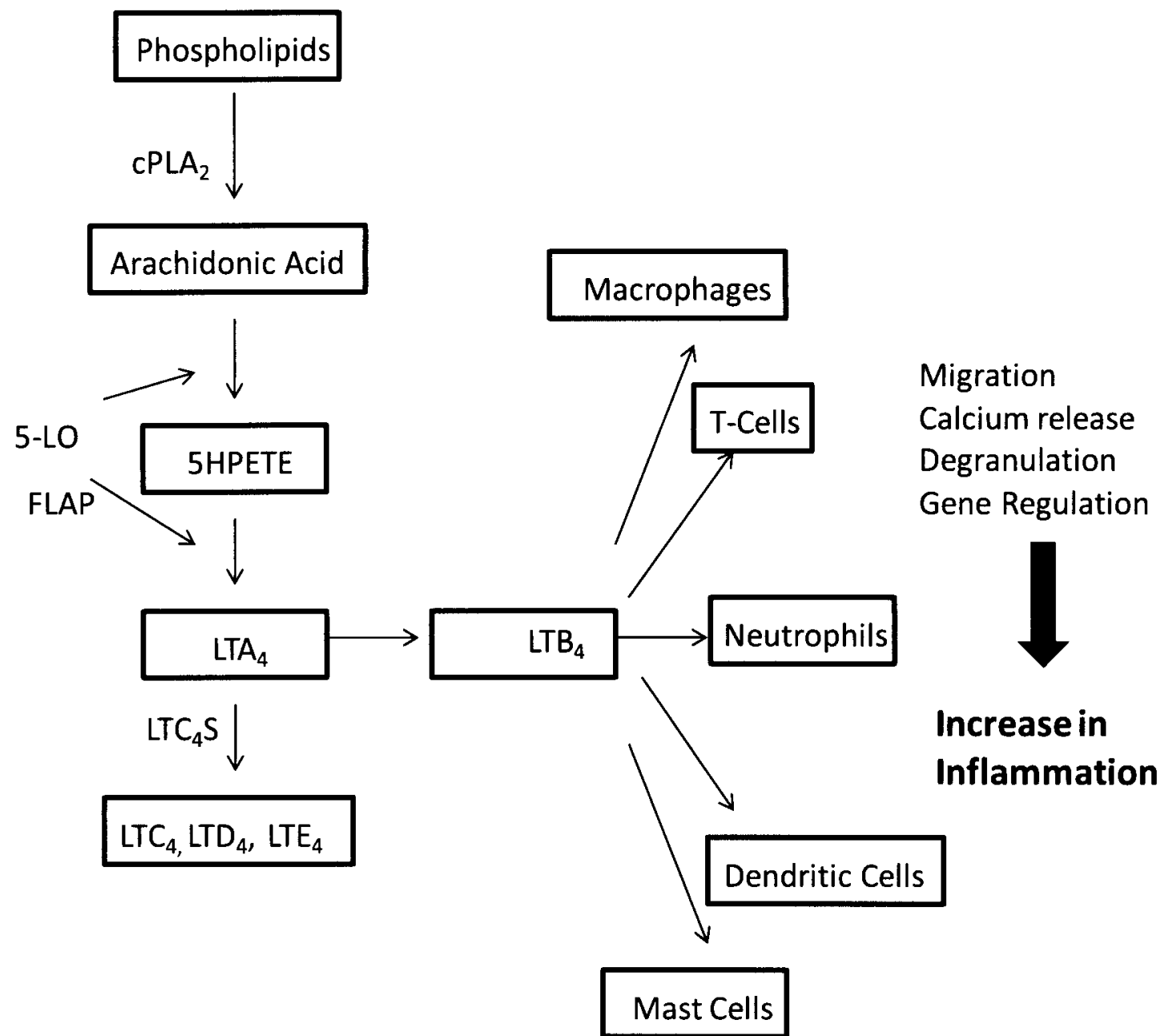

Figure 5.2. Pathways for synthesis of $\mathrm{LTB}_{4}$ (147)

The role of Leukotrienes (biologically active 5-lipoxygenase (5-LO) products of arachidonic acid metabolism) and their receptors (BLT1 and BLT2) are implicated in several inflammatory diseases like atherosclerosis (148-150). Of these, leukotriene $\mathrm{B}_{4}$ $\left(\mathrm{LTB}_{4}\right)$, a potent chemo-attractant for leukocytes, exerts its biological actions through specific G-protein coupled receptors (GPCR), BLT1 (a high affinity receptor) and BLT2 (low affinity receptor). $\mathrm{LTB}_{4}$ has direct involvement in various inflammatory diseases and Lipopolysaccharide (LPS) has the ability to induce the BLT1 expression level. Jala, et al. (151) identified mechanisms through which $\mathrm{LTB}_{4}$ could regulate atherogenesis. 
These studies outlined crucial new roles for leukotrienes in the recruitment of $T$ lymphocytes and in the development of atherosclerotic lesions, suggesting novel mechanisms for their actions. According to Fujihara, et al. (152), LPS is a major component in the baterial cell wall and exerts its functions via Toll-like receptors, which are known critical components in the promotion of inflammation. Since Leukotrienes and LPS have well-established roles in the pathophysiology of many inflammatory diseases, understanding the regulation of $\mathrm{LTB}_{4}$ and LPS in endothelial cells exposed to oscillatory shear stress can provide novel insights to unravel the role of $\mathrm{LTB}_{4}$ and its receptors under shear stress.

\subsubsection{Importance of bi-directional oscillatory WSS in atherosclerosis plaque formation}

Atherosclerosis is an inflammatory disease and mostly occurs in curved arterial vessels or bifurcations that are exposed to disturbed flow (low and oscillatory shear stress), whereas straight regions exposed to steady unidirectional stable flow are atheroprotective $(89,153)$. Wall shear stress was found to be a key factor in the development of atherosclerosis (154). A recent study showed that partial ligation of the mouse carotid artery causes a disturbed flow region with oscillatory shear that eventually induces atherosclerotic plaque formation (155). In another work, Tokuda, et al. (65) did three dimensional numerical simulation of blood flow in the aortic arch to understand the mechanism of stroke during cardiopulmonary bypass. Computational fluid dynamics was used to calculate wall shear stresses exerted by blood flow and they observed that depending on different flows at different locations of the arch, different pressures and 
wall shear stress were generated. The highest wall pressure was developed at the superior-posterior wall of the aorta below the orifice of the brachiocephalic artery whereas high velocity vortex was observed below the orifice of the brachiocephalic artery. The flows were turbulent throughout the posterior wall which flowed off into the left subclavian artery. The flow rates also were different in each of the branches of right subclavian, right common carotid, left common carotid and left subclavian artery. Also there is a strong correlation of oscillatory shear stress and formation of atherosclerotic plaque. It is therefore desirable to understand the blood flow pattern through the aortic arch and human carotid bifurcations where formation of atherosclerotic plaque is commonly observed. Figure 5.3 illustrated the fluid flow patterns in aortic arch, human carotid bifurcation and carotid artery. The figure (Figure 5.3A and Figure 5.3B) indicated that there are both curved and straight regions in aortic arch and carotid artery respectively. The pulsatile flow through the regions therefore exerts both oscillatory unidirectional and bidirectional shear stress. Oscillatory bidirectional shear is exerted in curved and arched regions whereas oscillatory unidirectional shear generates in straight regions. So it was desirable to understand the effects of directionality and magnitudes of exerted shear on atherosclerotic plaque formation which was investigated in current effort. Figure $4.3 \mathrm{C}$ therefore showed the different oscillatory shear (unidirectional and bidirectional) exposures on endothelial cells at different locations of a Petri dish, which were further analyzed to understand the relation between directional oscillatory shear and atherosclerotic plaque formation. Since directionality of shear was demonstrated to have an effect on cellular responses, the potential for a similar influence on atherogenic gene expression motivated this new study. The goal of this work was to determine the effects 
of pulsatile shear magnitude and direction on gene expression (I-CAM, E-Selectin, and IL-6) in the presence of $\mathrm{LTB}_{4}$ and LPS.

A

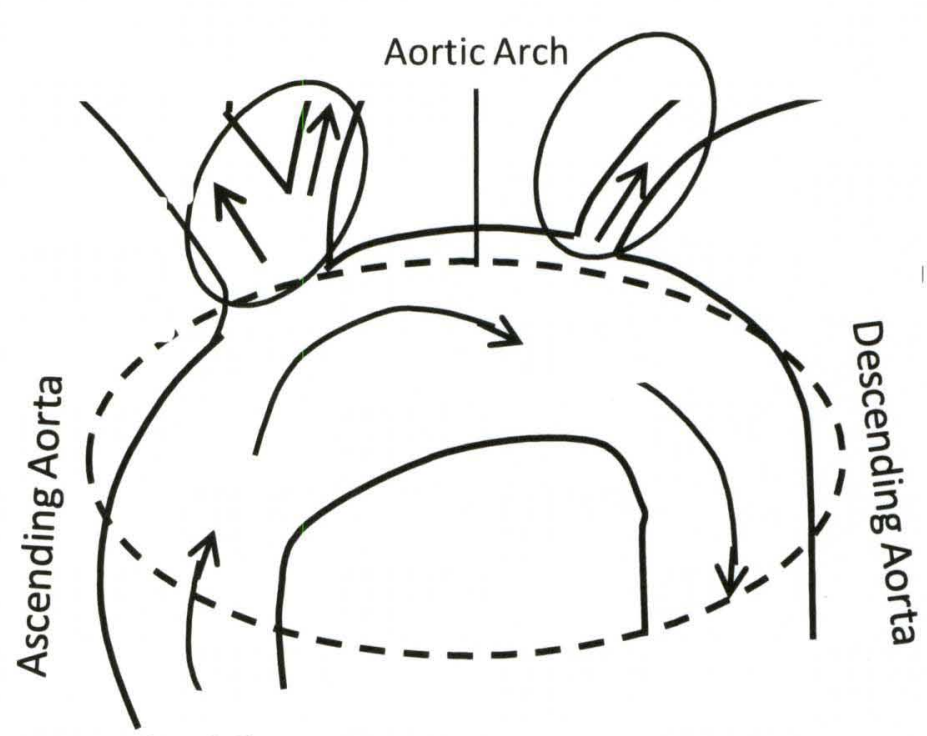

B

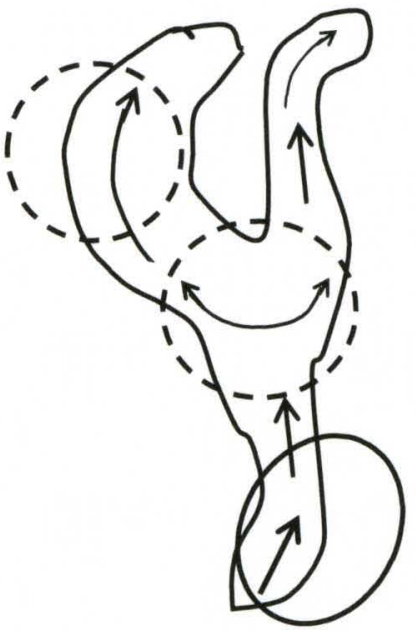

\section{Fluid flow}

C
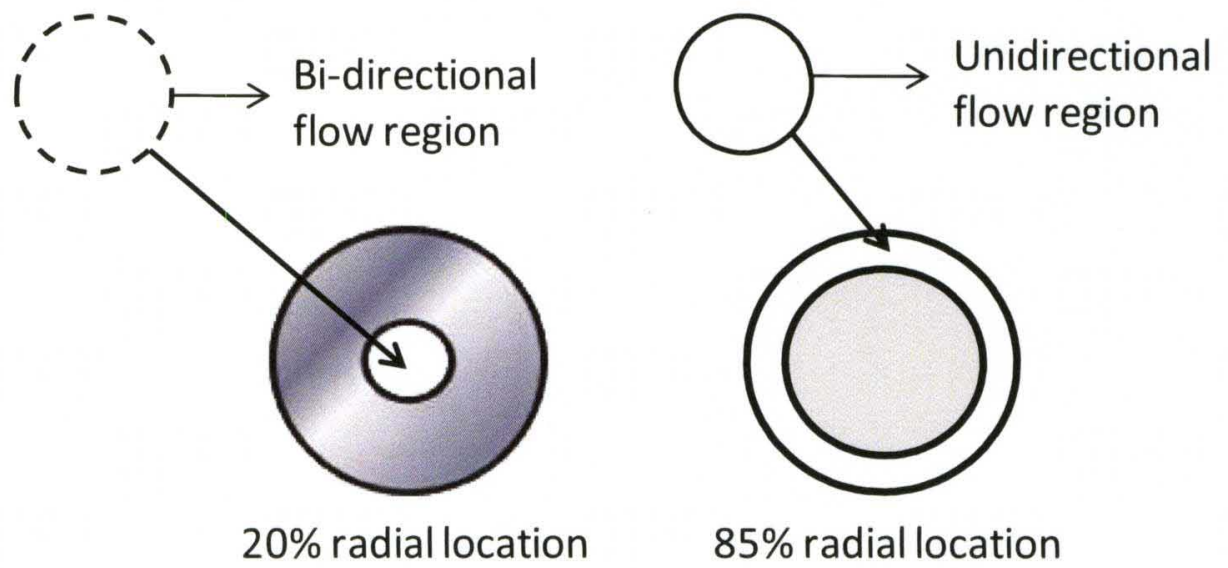

$85 \%$ radial location

Figure 5.3. Flow patterns of different fluid flows through different arteries. Figure 5.3A indicates the common pattern of human aortic arch and fluid flow through it. Figure 5.3B shows the fluid flow through human carotid arteries and Figure 5.3C indicates the bidirectional and unidirectional flow regions in experimental Petri-dish. 


\subsection{MATERIALS AND METHODS}

\subsubsection{Multiphase CFD Model and simulation process}

Details of the simulation protocol were described in Chapter two and three.

\subsubsection{Cell culture and Shear stress application}

Human umbilical vein endothelial cells (HUVECs) were maintained in $10 \mathrm{ml}$ culture medium (M-199) which was premixed with $10 \%$ fetal bovine serum, $10 \mathrm{ml}$ growth factor $(25 \mu \mathrm{g} / \mathrm{ml}) 1 \mathrm{ml}$ of heparin $(5 \mu \mathrm{g} / \mathrm{ml})$ and $5 \mathrm{ml}$ of Penicillin Streptomycin $(10000 \mu \mathrm{g} / \mathrm{ml})$. The cells were grown in Fibronectin-coated $(25 \mu \mathrm{g} / \mathrm{ml})$ cell culture dishes for 3 days at $37{ }^{\circ} \mathrm{C}, 5 \% \mathrm{CO}_{2}$ in a humidified $(80 \%)$ incubator. The cells were plated in new culture dishes $(5 \mathrm{~cm}$ diameter $\times 1.5 \mathrm{~cm}$ height) with a cell density of $0.20 \mathrm{million} / \mathrm{ml}$ containing $4 \mathrm{ml}$ of growth medium and allowed to adhere to the plate. To test the effect of different shear stress waveforms on gene expression, cells were grown at controlled radial locations by placing metal rings in the culture dish during the plating process. The metal rings were removed after 2 hrs, i.e., after cell adherence to the plates [1) over the entire dish, 2) inside a metal ring at $20 \%$ radial location, or 3) outside a ring at $85 \%$ radial location]. The dishes were then placed on an orbital shaker platform (Model-3500, Thomas Scientific, Swedesboro, NJ, USA) to generate pulsatile flow in the dishes. To determine the pulsatile shear effects on cell proliferation, morphology, and gene expression in the presence of $\mathrm{LTB}_{4}$ and LPS, two sets of plates containing HUVECs were grown at the three different radial locations in the presence of media containing either 
$\mathrm{LTB}_{4}(100 \mathrm{nM})$ or LPS $(100 \mathrm{ng} / \mathrm{ml})$ or both. One set of dishes was exposed to shear stress and another was grown under stationary conditions. The experiments were performed in triplicate.

\subsubsection{Quantitative Real Time Polymerase Chain Reaction (RT-PCR)}

The HUVECs were grown (in the whole dish or $20 \%$ or $85 \%$ radius) for $30 \mathrm{hrs}$ with and without shear stress exposure. These cells were induced with $\mathrm{LTB}_{4}(100 \mathrm{nM})$ or LPS $(100 \mathrm{ng} / \mathrm{mL})$ or $\mathrm{LTB}_{4}+\mathrm{LPS}$ for an additional $6 \mathrm{hrs}$ either at stationery or shear stress condition. The total RNA from these cells were isolated using RNeasy (Qiagen, Germantown, MD, USA) and followed by DNAse treatment (Ambion, Austin, TX, USA) to remove any genomic DNA contamination according to the manufactures protocol. The cDNA (Complementary DNA) was synthesized through a reverse transcription polymerase chain reaction (RT-PCR) process using random hexamers with TaqMan RT PCR kit (Applied Biosystems, Foster City, CA, USA). The synthesized cDNA was used to quantify the specific gene (I-CAM, E-Selectin and IL-6) expression by Real Time PCR with SyBR Green Reagent from Applied biosystems. The real time primers were obtained from Real Time Primers, LLC, Elkins Park, PA. The relative fold changes were calculated using the delta CT method by Livak et al. (156)

\subsubsection{Microscopy and image analysis}

Cell images were captured on a Nikon inverted microscope (Eclipse TE 300, Nikon Instruments Inc. Melville, NY, USA) at 10X magnification with a Cool Snap HQ digital B/W CCD camera (Roper Scientific, Trenton, NJ, USA). The cell numbers in 
those images were counted manually in Metamorph 4.6r5 software (Universal Imaging, Ypsilanti, MI, USA). The cell counts were then averaged over the three replicates to quantify proliferation. Further, cell areas were measured by using region measurement tools in Metamorph software. The cell images were taken at $20 \%$ (at $0.5 \mathrm{~cm}$ radial position) and $85 \%$ (at $2.1 \mathrm{~cm}$ radial position) radial locations. Twenty images around each radius were captured and total cells in each image were counted and averaged to quantify the cell proliferation and area.

\subsubsection{Statistical Analysis}

Unpaired t-tests were used to determine statistical significance of experiments which were performed using Graph Pad Prism Software (Graph Pad Software, La Jolla, CA, USA). Data were statistically significant when $\mathrm{p}<0.05$ and insignificant data are denoted by $p=N S$ (Not Significant).

Further, ANOVA analyses were used to identify the individual and interacting effects of DOSI, and shear magnitudes on cellular responses, including cell proliferation, morphology, and gene expressions as briefly discussed below

\subsubsection{ANOVA analysis to understand the effects of DOSI and shear magnitude on gene expressions}

Three-way ANOVA was used (157) to identify the effects on gene expression for a single orbital speed of DOSI and shear magnitude (treated as continuous variables) with four different treatments (treated as four different categories) from the endothelial cells of three individuals (replicates). The ANOVA model can be written as 


$$
Y_{i j k}=\mu+G_{i}+V_{j} I_{k}+(G V)_{i j}+(G I)_{i k}+D_{i j k}+M_{i j k}+\varepsilon_{i j k}
$$

where $\mu$ is the general mean effect, $G_{i}$ is the additional effect due to the ith gene, $V_{j}$ is the additional effects due to the jth treatment and $I_{k}$ is the additional effect due to the kth individual (replicate). $(G V)_{i j}$ is the interaction effect between the ith gene and jth treatment and $(G I)_{i k}$ is the interaction effect between the ith gene and kth individual (replicate). $D_{i j k}$ and $M_{i j k}$ are respectively the values of DOSI and shear magnitude for the ith gene, jth treatment and kth individual. $\epsilon$ are the random error component.

\subsubsection{ANOVA analysis to understand the effects of DOSI and shear magnitude on cell proliferation and morphology in the presence of $L T B_{4}$ and LPS}

Three-way ANOVA was also used to identify the effects on cell proliferation for a single orbital speed of DOSI and shear magnitude (treated as continuous variables) with four different treatments (treated as four different categories) with three replicates.

The following ANOVA model is used to evaluate our desired interaction between DOSI and shear magnitude

$$
Y_{i j k}=\mu+D_{i}+T_{j}+M_{i}+(D T)_{i j}+(M T)_{i j}+\varepsilon_{i j k}
$$

where $Y_{i j k}$ is the cell proliferation corresponding to the ith DOSI value, jth treatment variety and kth replicate. $\mu$ is the general mean effect, $D_{i}$ and $M_{i}$ are the ith values of DOSI and shear magnitude respectively. $(D T)_{i j}$ and $(M T)_{i j}$ are the respective interaction effects of DOSI and shear magnitude with treatment variety. $\varepsilon_{i j k}$ is the random error component for the ith DOSI value, jth treatment variety and kth replicate. 


\subsection{RESULTS AND DISCUSSIONS}

\subsubsection{Determination of WSS by CFD and Estimation of DOSI}

CFD was used to determine oscillatory WSS exerted by fluid flow on HUVECs grown on the bottom of an orbiting Petri-dish at different radial locations at $100 \mathrm{rpm}$ orbital speed. The resultant WSS was determined from simulations to be more uniform at $20 \%$ radial location, and less uniform at $85 \%$ radial location for $100 \mathrm{rpm}$ orbital speed. The highest WSS was observed near the vertical wall of the dish and lower shear was observed near the center of the dish (Figure 5.4A). At $100 \mathrm{rpm}$, WSS ranged from 0-9 dyne $/ \mathrm{cm}^{2}$ at $85 \%$ of the radius, and $0-1 \mathrm{dyne} / \mathrm{cm}^{2}$ at $20 \%$ radial location, respectively. An example plot of tangential vs. radial WSS from the CFD results, with data taken from 24 evenly spaced times throughout one complete orbit, is illustrated in Figure 5.4B. Shear was bi-directional (radial and tangential) at $20 \%$ of the radius and was mostly tangential at $85 \%$ of the radius. DOSI was calculated (Table 5.1) on the basis of principal axes, derived from these two shear components, which was described in the Results section of Chapter Three. Figure 5.4C shows the plots of WSS of $1^{\text {st }}$ principal axis vs. WSS of $2^{\text {nd }}$ principal axis at different radial locations ( $20 \%$ and $85 \%)$ for $100 \mathrm{rpm}$ orbital radius. DOSI was low at $20 \%$ radial location, where oscillation of shear was nearly the same in both directions. At increasing radius, it was observed that tangential shear was more oscillatory and radial shear was less oscillatory, which caused DOSI to become higher. At $85 \%$ of the radius, radial OSI was much larger than tangential OSI, causing DOSI to approach unity. The results highlight the usefulness of CFD in determining oscillatory WSS and eventually helped to generate DOSI values, which were used to determine the effects of directionality of oscillatory shear on endothelial cellular responses. 
A.
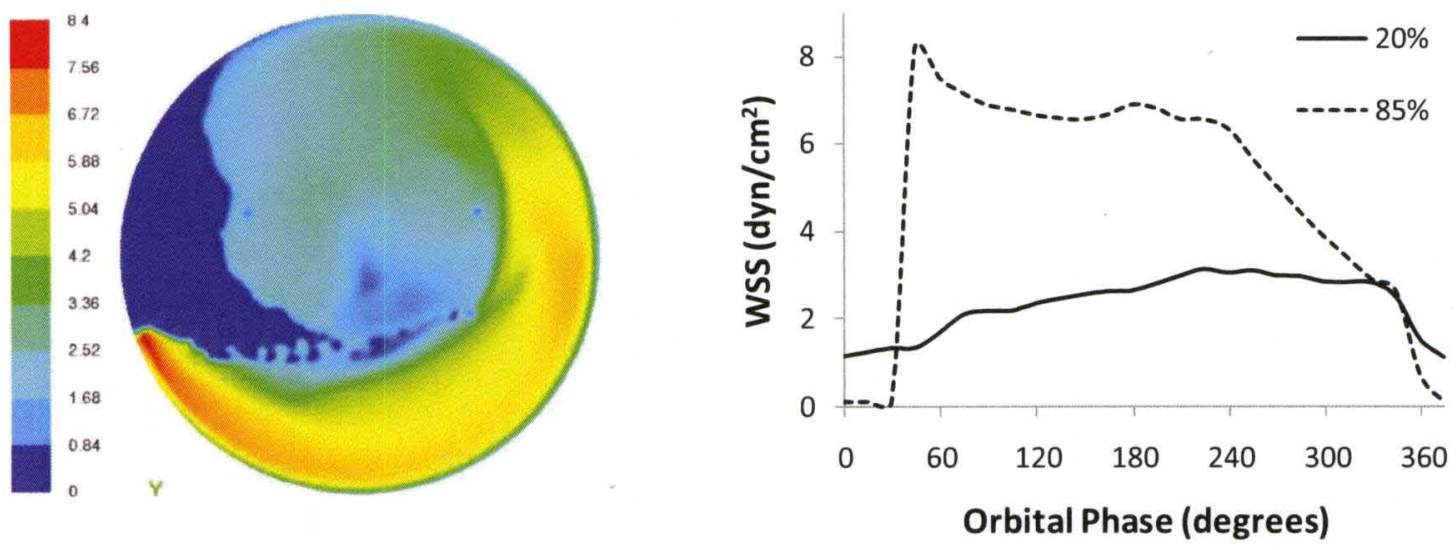

B.

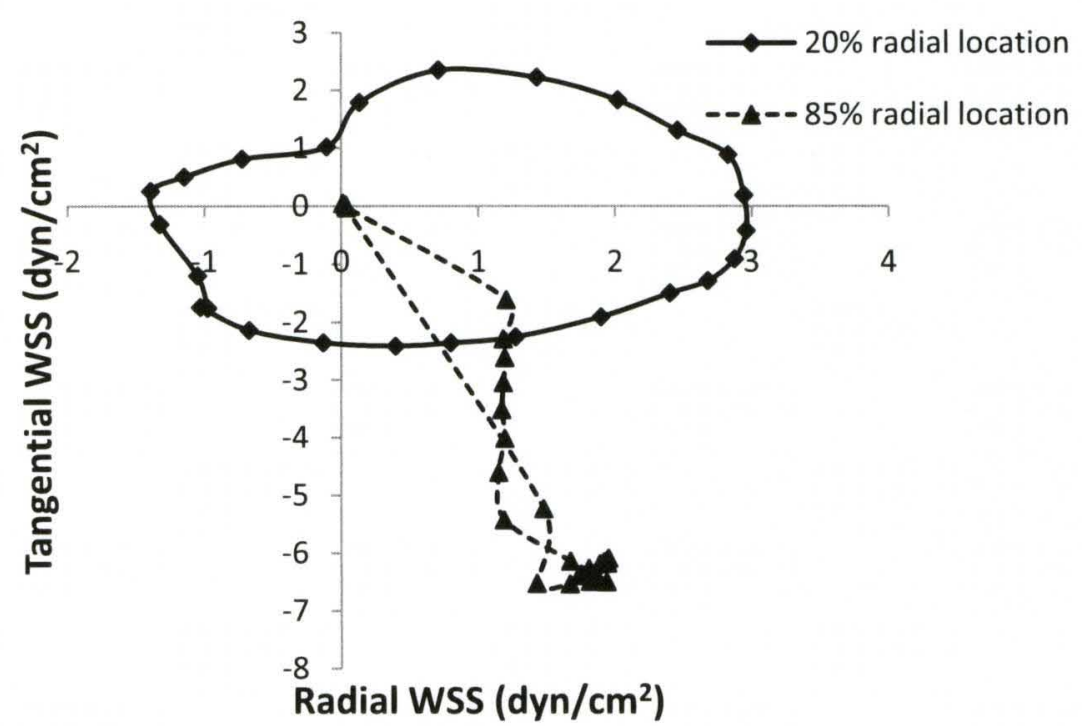


C.

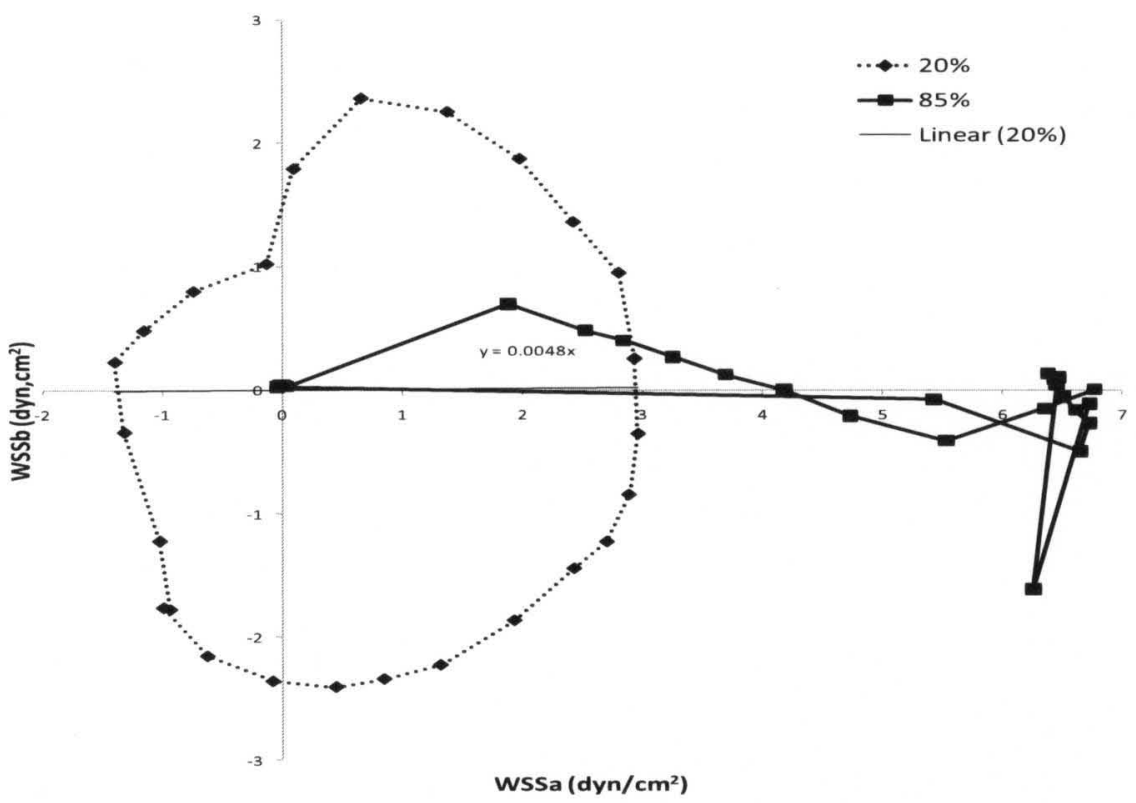

Figure 5.4. Resultant wall shear stress contours $\left(\mathrm{dyn} / \mathrm{cm}^{2}\right)$ at the bottom surface of the dish and shear waveforms at different radial locations $(20 \%$ and $85 \%)$ at an orbital speed of $100 \mathrm{rpm}$ (Figure 5.4A). Figure 5.4B plots tangential versus radial wall shear stress at different radial locations $(20 \%$ and $85 \%)$ at $100 \mathrm{rpm}$ orbital speed. Figure $5.4 \mathrm{C}$ shows the plots of $1^{\text {st }}$ and $2^{\text {nd }}$ principal axes WSS components.

Table 5.1. DOSI at $100 \mathrm{rpm}$ orbital speeds and at radial locations of $20 \%, 60 \%$, and $85 \%$.

\begin{tabular}{ccccc}
\hline Orbital speed (rpm) & Radial location (\%) & OSI $_{\text {tan }}$ & OSI $_{\text {rad }}$ & DOSI \\
\hline \multirow{2}{*}{100} & 20 & 0.892 & 0.620 & 0.305 \\
& 60 & 0.917 & 0.075 & 0.918 \\
& 85 & 0.986 & 0.006 & 0.994 \\
\hline
\end{tabular}




\subsubsection{Effects of DOSI and shear magnitude on cell proliferation and cell area in the}

presence of Leukotriene $\mathrm{B}_{4}\left(\mathrm{LTB}_{4}\right)$ and Lipopolysaccharide (LPS)

HUVECs were grown in the presence of $\operatorname{LTB}_{4}(100 \mathrm{nM})$ and/or LPS $(100 \mathrm{ng} / \mathrm{ml})$ and exposed to oscillatory WSS by placing them on an orbiting Petri-dish at $100 \mathrm{rpm}$ orbital speed. After 36 hours of shear exposure, the cell images were captured using the inverted microscope. Representative cell images are shown in Figure 5.5, and cell proliferation and area values are compared in Figure 5.6. Cell proliferation for untreated cells was significantly higher compared to unstressed controls at $20 \%$ radial location (low DOSI) and significantly lower at $85 \%$ radial location (high DOSI). Though cell proliferations at different shear exposure were compared to untreated and unstressed control cell lines as illustrated in Figure 5.6A, different pair-wise statistical comparisons were done thereafter to understand the significant effects of different shear stress levels on cell proliferations under different treatments at different radial locations. It showed that the cells treated with $\mathrm{LTB}_{4}$ exhibited significantly greater proliferation at both $20 \%$ and $85 \%$ radial locations than untreated controls both with and without shear exposure. Interestingly, $\mathrm{LTB}_{4}$ overcame the negative effect of shear stress to produce increased cell proliferation at $85 \%$ radial location (Figure 5.5B, right panel and Figure 5.6A) compared with untreated cells (Figure 5.5A, right panel and Figure 5.6A). In contrast, proliferation for unstressed cells treated with LPS was increased, but changed insignificantly for stressed cells. LPS reduced the effect of $\mathrm{LTB}_{4}$ when cells were treated with both. In fact, proliferation of cells treated with LPS and $\mathrm{LTB}_{4}$ at $85 \%$ radius was less than untreated controls. 
Figure 5.6B shows the effects of different shear stress on cell morphology at different radial locations with treatment of $\mathrm{LTB}_{4}$ and LPS. Results indicate that the area of untreated cells did not change significantly at $20 \%$ radial location (low DOSI) compared to unstressed controls, whereas it increased significantly at $85 \%$ radial location (high DOSI). Different pair-wise statistical comparison showed that unstressed cells treated with $\mathrm{LTB}_{4}$ increased in cell area compared to untreated and unstressed control cell lines, but cells under shear stress did not show significant change in their cell area. However, there were significant decreases in area of cells treated with $\mathrm{LTB}_{4}$ both at $20 \%$ and $85 \%$ radial locations, when compared to the unstressed cells treated with $\mathrm{LTB}_{4}$. For cells that were treated with either LPS alone or both LPS and $\mathrm{LTB}_{4}$, cell area increased significantly regardless of the shear applied. The above results indicate the effects of different DOSI and shear magnitudes on cell proliferation and morphology at different radial locations in the presence of atherogenic regulators like $\mathrm{LTB}_{4}$ and LPS. While $\mathrm{LTB}_{4}$ and LPS have proven effects on cellular responses like proliferation and morphology, the data showed that the directionality (DOSI) of shear and magnitude of shear has significant impacts in altering those responses. 


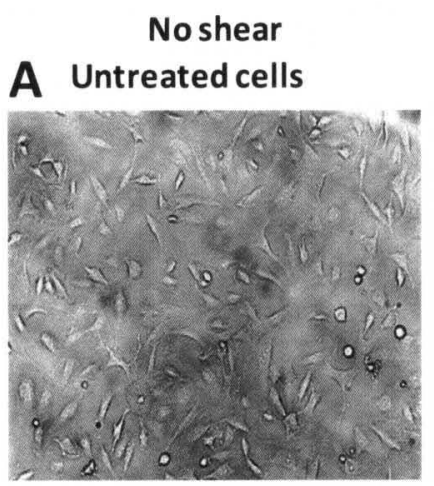

A Untreated cells
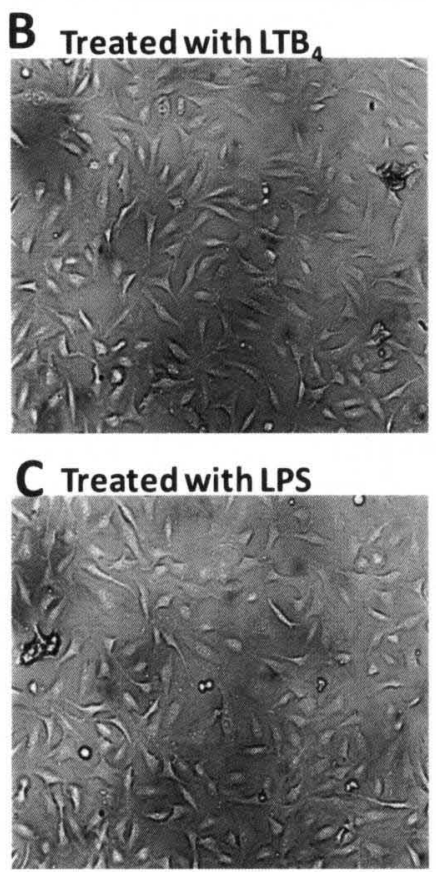

\section{Treated with $\mathrm{LTB}_{4}$ and LPS}

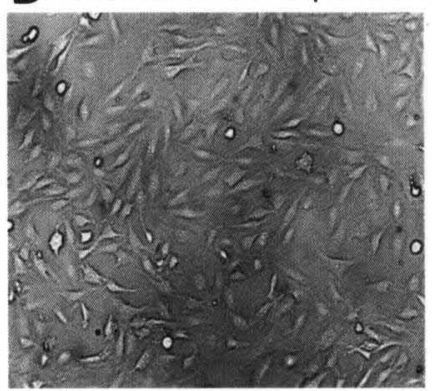

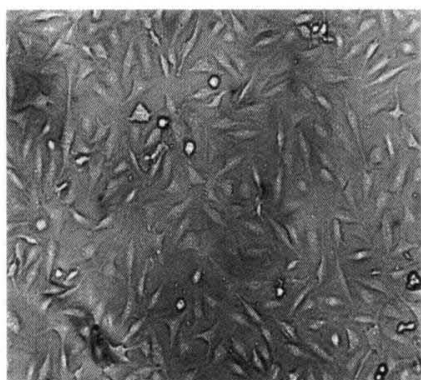
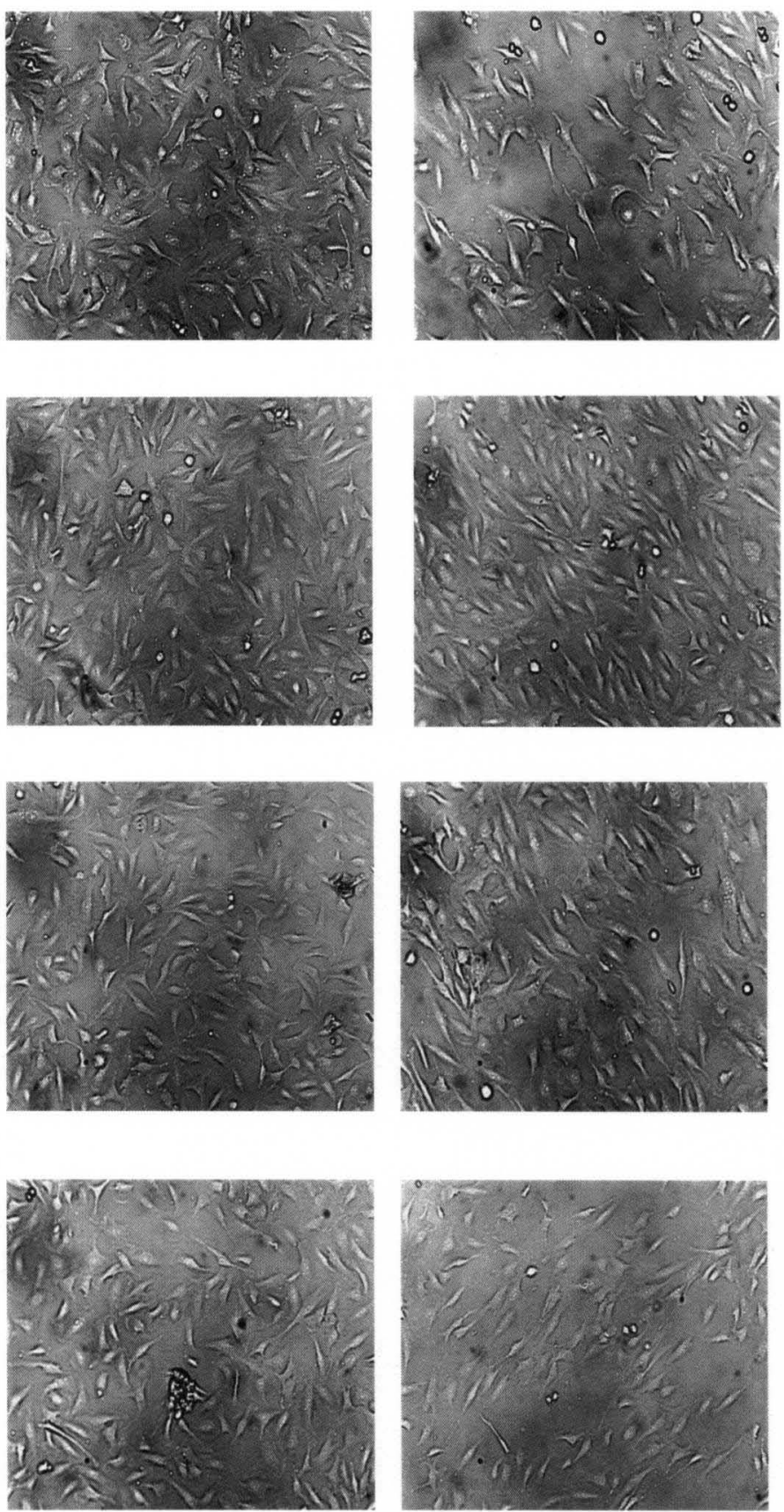

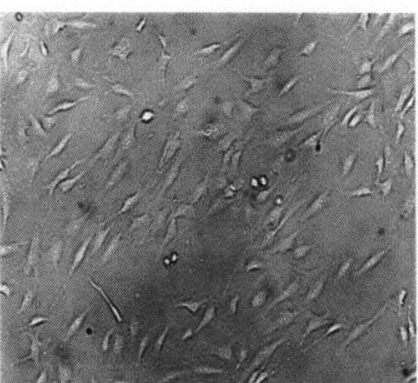

Figure 5.5. Cell images at two locations and DOSI levels at $100 \mathrm{rpm}$ orbital speed. Panel 5.5A shows untreated cells. Panels 5.5B, 5.5C, and 5.5D show cells treated with $\mathrm{LTB}_{4}$, LPS, and $\mathrm{LTB}_{4}+\mathrm{LPS}$, respectively. 


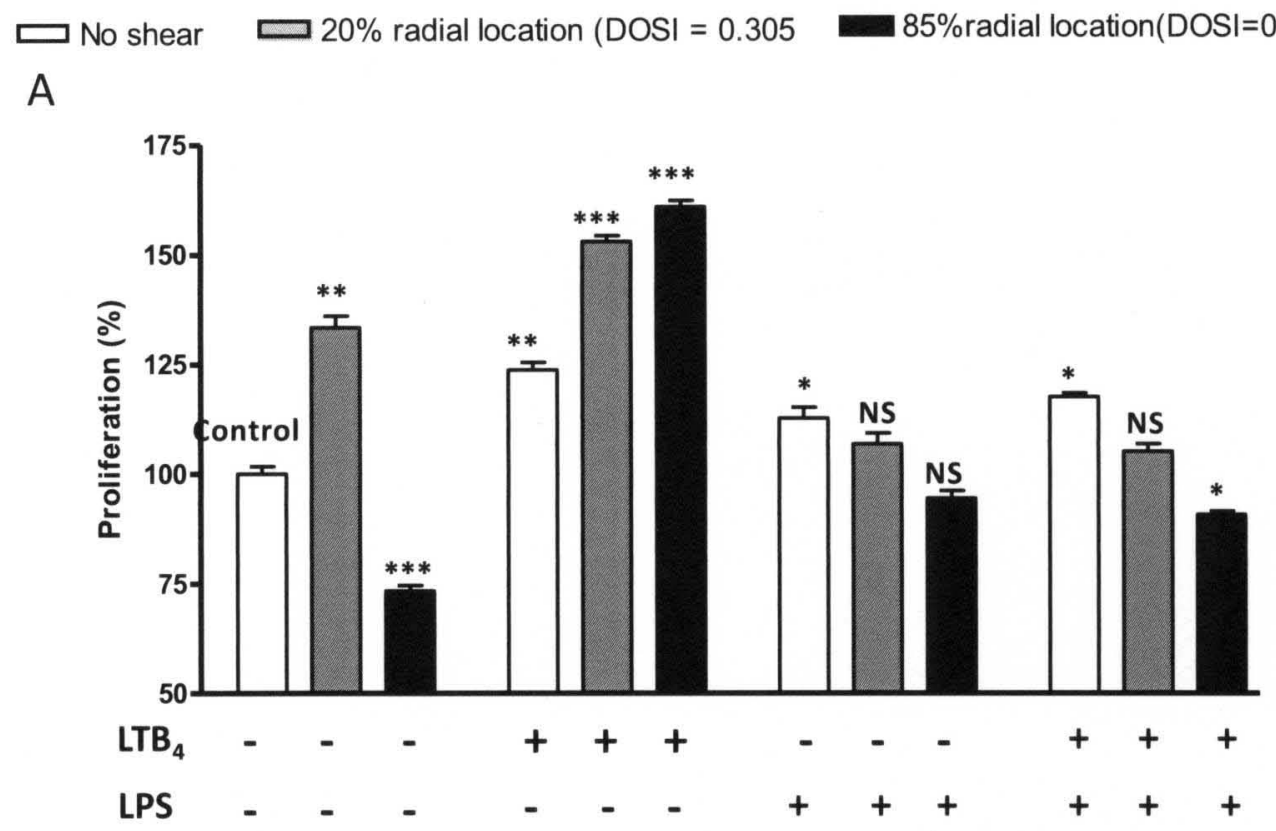

B

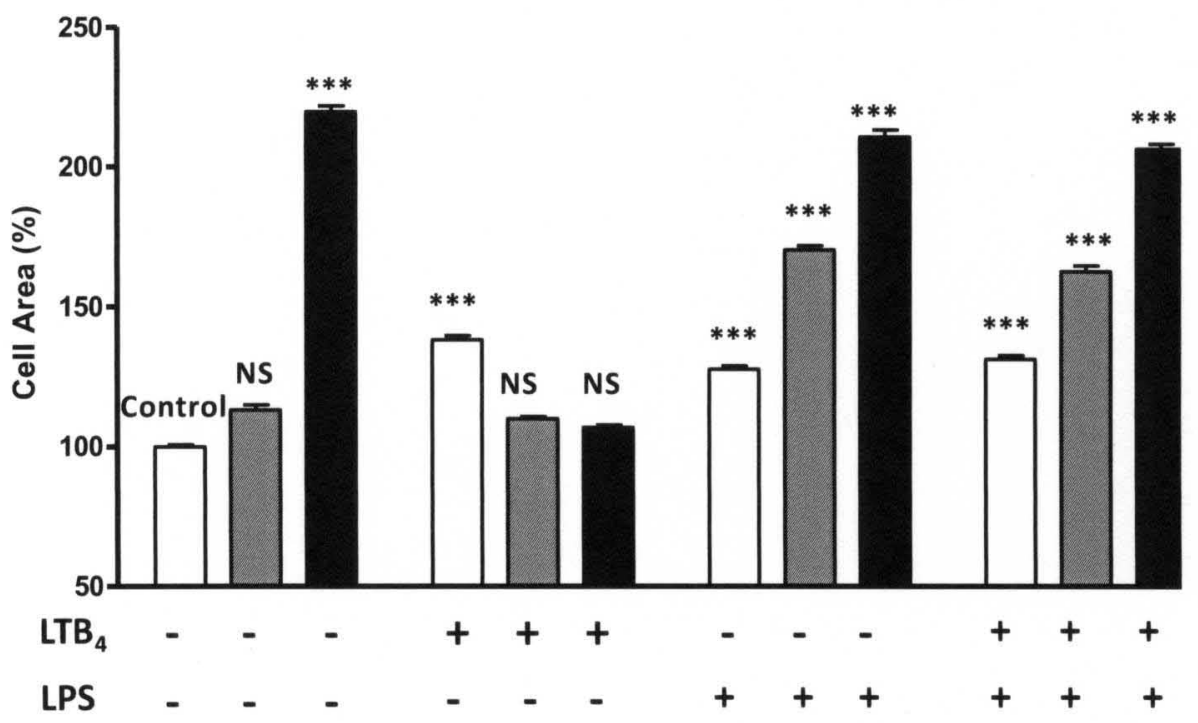

Figure 5.6. Cell proliferation and area in the presence of $\mathrm{LTB}_{4}$ and LPS under different DOSI levels and shear magnitudes at two radial locations ( $20 \%$ and $85 \%)$ at $100 \mathrm{rpm}$ orbital speed. DOSI $\sim 0$ near the center (at 20\%) and DOSI $\sim 1$ near the side wall (at $85 \%)$. Data are expressed as mean $\pm \mathrm{SD}$. Statistical significances are expressed as $* P<$

$$
0.05,{ }^{* *} P<0.01 \text {, and }{ }^{* * *} P<0.001 \text { respectively. }
$$




\subsubsection{Individual and interacting effects of DOSI and shear magnitude on cell proliferation and area in the presence of $L T B_{4}$ and LPS by ANOVA technique}

The effects of directionality and magnitudes of shear on HUVECs proliferation and morphology were analyzed by applying ANOVA statistical distribution. Analysis of variance (ANOVA) for these trials for the model in Equation (5.2) is shown in Table 5.2. The table indicates that all individual and interacting effects of DOSI, shear magnitude and treatment type on cell proliferation were statistically significant. From the table, it was evident that all the interactions between DOSI, treatment, and magnitudes (D:T, D:M, M:T) were statistically significant, implying that the effects of DOSI and shear magnitudes on cell proliferation changes substantially over the four different treatments (untreated, treated with $\mathrm{LTB}_{4}$, treated with LPS, and treated with $\mathrm{LTB}_{4}$ and LPS). Furthermore, the Table showed that individual effects of DOSI on average cell area were significant and all the interacting effects of DOSI, treatment, and magnitudes (D:T, D:M, M:T) are statistically significant, indicating that DOSI influences the cell morphology significantly. 
Table 5.2. ANOVA analysis of cell proliferation and cell area responses in the presence of $\mathrm{LTB}_{4}$ and LPS to DOSI and shear magnitude.

\begin{tabular}{ccccc}
\hline & \multicolumn{2}{c}{ Effects on Cell Proliferation } & \multicolumn{2}{c}{ Effects on cell area } \\
Parameters & Degree of Freedom & Significance (P) & Degree of Freedom & Significance (P) \\
\hline DOSI (D) & 1 & $<0.0001$ & 1 & $<0.0001$ \\
Shear Magnitude (M) & 1 & $<0.0001$ & 1 & NS \\
Treatment (T) & 3 & $<0.0001$ & 3 & $<0.0001$ \\
DT & 3 & $<0.0001$ & 3 & $<0.0001$ \\
MT & 3 & $<0.0001$ & 3 & $<0.0001$ \\
DM & 1 & $<0.0001$ & 1 & NS \\
Residuals & 84 & & 84 & \\
\hline
\end{tabular}

\subsubsection{Effects of DOSI and shear magnitude on atherogenic gene expressions in the presence of $L T B_{4}$ and $L P S$}

The effects of different DOSI and shear magnitudes on the regulation of atherogenic genes like I-CAM, E-Selectin and IL-6 were analyzed in the presence of $\mathrm{LTB}_{4}$ and LPS at $20 \%$ and $85 \%$ radial locations. Real time PCR was performed to understand the different effects of directionality and shear magnitudes on those genes at mRNA level. There was a significant increase in the expression of ICAM-1 with increasing DOSI and shear magnitude (Figure $5.7 \mathrm{~A}, 1^{\text {st }}$ panel). Interestingly, the shearinduced increase in ICAM-1 expression was eliminated by the addition of $\mathrm{LTB}_{4}$ (Figure $5.7 \mathrm{~A}, 2^{\text {nd }}$ panel). Further, LPS significantly up-regulated ICAM-1 expression, which confirms previously reported data (158), and LPS-induced ICAM-1 expression was significantly decreased upon exposure to higher DOSI (0.994) at $85 \%$ radial location 
(Figure 5.7A, $3^{\text {rd }}$ panel). Also, LPS-induced expression of ICAM-1 is reduced in the presence of $\mathrm{LTB}_{4}$ (Figure $5.7 \mathrm{~A}, 4^{\text {th }}$ panel).

Without treatment, the mRNA levels of E-Selectin decreased significantly at $85 \%$ radial location $(\mathrm{DOSI}=0.994)$, but not at $20 \%(\mathrm{DOSI}=0.305)\left(\right.$ Figure $5.7 \mathrm{~B}, 1^{\text {st }}$ Panel $)$. In the presence of $\mathrm{LTB}_{4}$, E-Selectin increased significantly both at $20 \%$ and $85 \%$ radial locations (Figure 5.7B, $2^{\text {nd }}$ panel). LPS alone up-regulated E-Selectin compared to untreated control for all shear conditions, but the expression had a significant decreasing trend with increase of DOSI levels (Figure $5.7 \mathrm{~B}, 3^{\text {rd }}$ panel). LPS and $\mathrm{LTB}_{4}$ also increased E-Selectin, but the increase was greater at $20 \%$ radial location than either no shear or $85 \%$ radial location.

The expression of IL-6 was significantly increased at both radial locations compared to control, but the expression at $85 \%$ radial location (higher DOSI) was significantly lower than that at $20 \%$ radial location (lower DOSI) (Figure $5.7 \mathrm{C}, 1^{\text {st }}$ panel). Treatment with $\mathrm{LTB}_{4}$ and LPS and their combination increased IL-6 expression (Figure $5.7 \mathrm{C}, 2^{\text {nd }}, 3^{\text {rd }}$, and $4^{\text {th }}$ panel), but did not change the trend of maximum expression at $20 \%$ radial position. 
Control (No shear, no treatment) $\square$ No shear, treated with LTBand/or LPS

\section{A I-CAM}
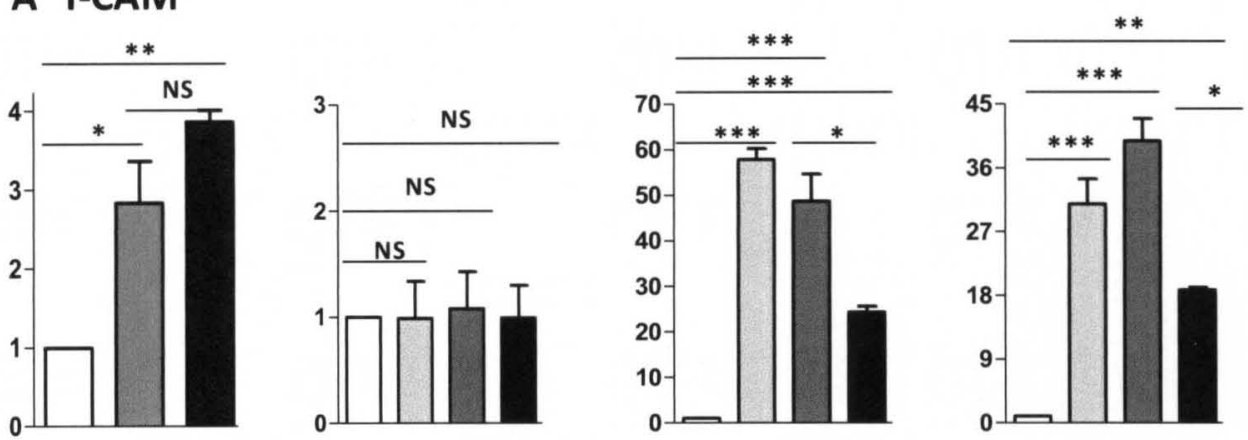

\section{B E-Selectin}
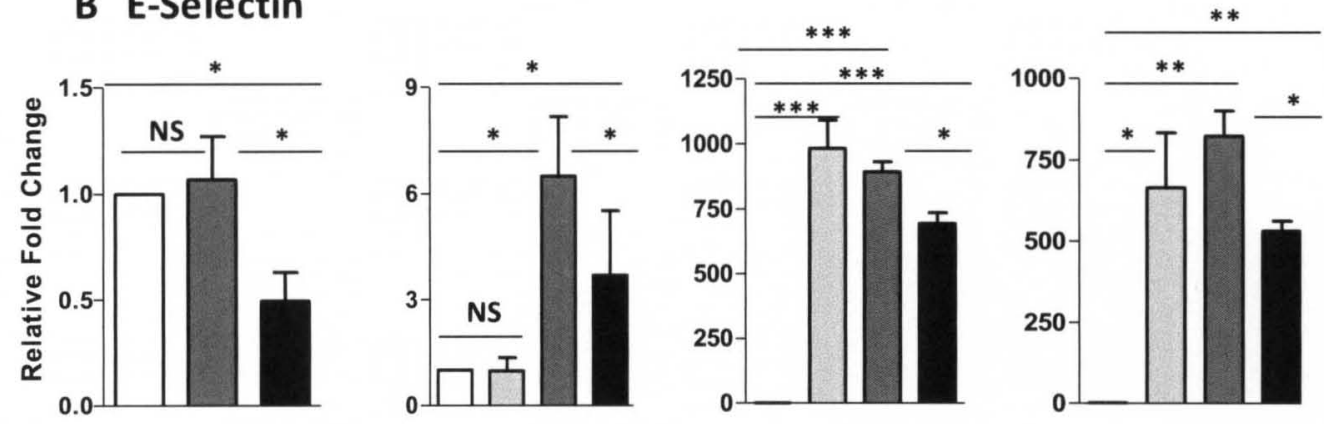

\section{IL-6}
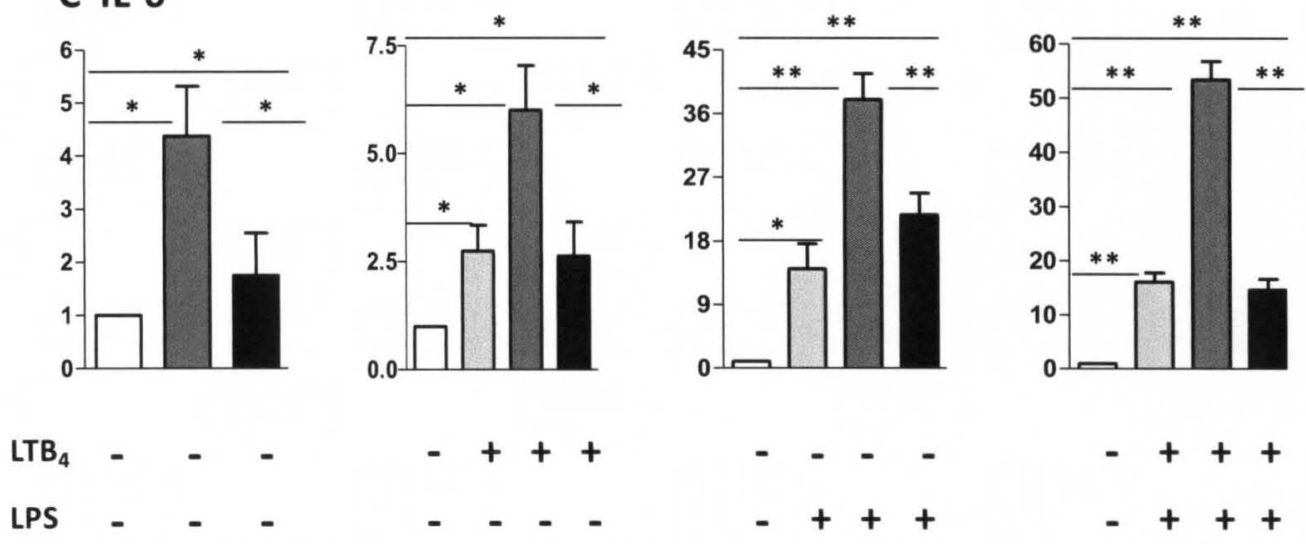

Figure 5.7. Effects of DOSI and shear magnitudes on gene expressions (I-CAM, E-

Selectin, and IL-6) at mRNA level at different radial locations (20\% and $85 \%)$ at orbital speed of $100 \mathrm{rpm}$. Data are expressed as mean \pm SD. Statistical significances are expressed as $* P<0.05,{ }^{* *} P<0.01$, and ${ }^{* * *} P<0.001$ respectively. 


\subsubsection{Individual and interacting effects of DOSI and shear magnitude on gene}

expressions in the presence of $L T B_{4}$ and LPS by ANOVA technique

The ANOVA technique was used in Table 4.3 by the model explained in equation (5.1) to investigate the individual and interacting effects of DOSI, shear magnitudes, and treatment types (untreated, treated with $\mathrm{LTB}_{4}$, treated with LPS, and treated with $\mathrm{LTB}_{4}$ and LPS) on gene expressions at mRNA level. The table indicates that the main effects of gene treatment variety and DOSI were highly significant. The significance of DOSI was further reinforced by the significance of its interaction of gene effect (D:G in the table). Further, there was highly significant interaction between gene and treatment variety (G:T in table). This indicated that gene expression changes substantially over the four different treatment varieties. It was interesting that the essence of this fact was captured by the significance of the interaction between DOSI and treatment variety (D:T). This indirectly proved that DOSI is an important factor which significantly causes the variation of the gene expression over the four different treatment varieties. Thus, it can be concluded that DOSI nicely captures the data generating mechanism of the response which eventually indicated that the directionality of shear has significant impact on gene expression with different treatment. 
Table 5.3. ANOVA analyses of gene expression responses to DOSI, shear magnitude, treatment, and replicate.

\begin{tabular}{ccc}
\hline Parameters & Degrees of Freedom & Significance (P) \\
\hline Genes (G) & 2 & $<0.001$ \\
Treatment (T) & 3 & $<0.001$ \\
Replicates (I) & 2 & NS \\
DOSI (D) & 1 & $<0.01$ \\
Shear Magnitude (M) & 1 & NS \\
GT & 6 & $<0.001$ \\
GI & 4 & NS \\
DG & 2 & $<0.001$ \\
DT & 3 & $<0.05$ \\
DM & 1 & NS \\
GM & 2 & NS \\
TI & 6 & NS \\
TM & 3 & NS \\
MI & 2 & NS \\
DI & 2 & NS \\
Residuals & 80 & \\
\hline & &
\end{tabular}




\subsubsection{CONCLUSION}

Extensive computational, experimental and statistical analyses were done in this effort to understand how different directional fluid flows affect endothelial cellular responses and atherosclerotic disease progression in the presence and absence of inflammation inducing molecules such as $\mathrm{LTB}_{4}$ and LPS in an in-vitro cell culture environment. It was observed in a previous study (159) that bidirectional shear (radial and tangential shear of similar magnitudes, DOSI $\sim 1$ ) occurs near the center of an orbiting dish, and unidirectional (tangential, DOSI $\sim$ ) near the vertical wall of the dish. These differences in directionality of shear influence cell morphology, including proliferation, area, shape index and orientation. The current study was performed to extend understanding of the effects of directionality of shear to cell function, in particular, expression of genes involved in atherosclerosis. The effects of directionality of shear on cell morphology were first confirmed in the presence of atherogenic regulators LPS and $\mathrm{LTB}_{4}$. It was observed that Cell proliferation increased due to shear at low DOSI and decreased at high DOSI, when both conditions were compared to no shear stress. However, incubation of cells in the presence of $\mathrm{LTB}_{4}$ increased cell proliferation regardless of shear. It was previously shown that inhibitors of $\mathrm{LTB}_{4}$ receptor 1 (BLT1) cause a decrease in proliferation for pancreatic cancer cell lines (160) and LTB4 induces cell proliferation of neural stem cells (NSCs) (161), Rat-2 fibroblast cells (162) as well as T-cells in a IL 2 dependent manner (163). This is the first report to suggest that the $\mathrm{LTB}_{4}$ induces cell proliferation of endothelial cells. The molecular mechanisms involved in this process are yet to be explored. LPS tended to rescind both effects, reflecting the previously observed apoptotic effects of LPS on endothelial cells. 
Cell morphology is regulated by several cellular molecular and physical processes including cell division, cell death, necrosis, apoptosis and stress which lead to changes in the cell shapes. The current study investigated the effects of shear stress on cell morphology in the presence and of absence of $\mathrm{LTB}_{4}$ and LPS. The results presented here demonstrated that cell area was significantly increased by unidirectional high shear near the side wall compared to that at low bidirectional shear near the center of the dish. The presence of $\mathrm{LTB}_{4}$ compensated for these effects at both DOSI levels suggesting a critical role of $\mathrm{LTB}_{4}$ in cell morphology maintenance. This data also supports the fact that $\mathrm{LTB}_{4}$ induces cell proliferation, which requires a healthy status of cells. It was previously shown that LPS induces changes in cell morphology for stationary conditions (164). Here, the data shows that LPS increased cell area regardless of shear level even in the presence of $\mathrm{LTB}_{4}$ indicating LPS is one of the major regulators of cell morphology. The results also demonstrated that a decrease in cell proliferation in the presence of LPS under unidirectional high shear (near the side wall) results in an increase in cell area. Similar effects were also observed in the presence of $\mathrm{LTB}_{4}$. The mechanisms underlying these effects are yet to be explored.

Further, to understand both individual effects and the effects of interactions between DOSI and shear magnitudes on cellular responses (proliferation, morphology, and gene expressions) treated with LTB $_{4}$ and LPS, the three-way ANOVA statistical analyses was used. ANOVA statistical analyses revealed that DOSI played an important role in altering responses of cells treated with $\mathrm{LTB}_{4}$ and LPS. From Table 2, it was evident that all individual and interacting effects involving DOSI, were significant, except for the interaction with shear magnitude for cell area. 
Binding and recruitment of circulating leukocytes to the vascular endothelium are key steps in atherosclerotic lesion development and are mediated by several cellular adhesion molecules that are expressed on the surface of vascular endothelial cells. Intracellular adhesion molecule -1 (I-CAM1), endothelial-leukocyte adhesion molecule-1 (E-Selectin) and interleukin-6 (IL-6) play important roles in atherosclerotic lesion development (127-132). It is also well known that ICAM-1, vascular cell adhesion molecule-1 (VCAM-1), and E-Selectin play critical roles in the development of atherosclerosis by sequestering the circulating leukocytes $(133,134)$. These molecules mediate these actions by supporting adhesive interaction and affecting cell signaling $(135,136)$. Interleukin-6 (IL-6), a pro-inflammatory cytokine, contributes to atherosclerotic plaque development and plaque destabilization (137). Transmission of those shear stress signals throughout the vascular cell involves cytoskeletal and biochemical elements and influences gene expression, including I-CAM1, E-Selectin and IL-6. Nagel, et al. (138) found that cultured human umbilical vascular endothelial cells (HUVECs) exposed to laminar shear stresses of $25-46 \mathrm{dyn} / \mathrm{cm}^{2}$ up-regulated ICAM-1 expression, whereas E-Selectin and VCAM-1 were not up-regulated at any time point examined. This selective regulation of adhesion molecule expressions may contribute different levels of endothelial gene expression and may be considered a relevant stimulus in inflammation and atherosclerosis. Here, the regulation of ICAM, E-selectin and IL-6 in the presence of $\mathrm{LTB}_{4}$ and LPS was determined at various shear/DOSI conditions. The results presented here provide clues for changes in proliferation at various DOSI levels, where IL-6 increased at low DOSI and decreased at higher DOSI is suggestive of the role of IL-6 in cell proliferation. The effects of DOSI, LTB4 and LPS are very influential in 
regulating I-CAM, E-selectin and IL-6 providing clues about disease development. The data clearly demonstrate the role for individual effects on the expression patterns of these genes in HUVECs (Fig 4.7). ANOVA also indicated that the expressions of atherogenic genes I-CAM1, E-Selectin and IL-6 were influenced by DOSI. Table 4.3 indicates that the individual effect of DOSI was significant, as were its interactions with the other factors with significant individual effects - gene and treatment. The results indicated that there were significant alterations in expression of those genes under different DOSI and shear magnitude levels. It eventually reveals significant impacts of directionality and magnitudes of exerted shear in the formation of atherosclerotic plaque, especially where the flow is not unidirectional and is pulsing in nature.

While the significant impacts of the directionality of oscillatory shear on cellular morphology were demonstrated in previous studies (159), this investigation suggests that the directionality of shear modulates atherosclerotic gene expression and, thus, influences the formation of atherosclerotic plaque. 


\section{CHAPTER SIX}

\section{CONCLUSION \& RECOMMENDATIONS}

\subsection{CONCLUSION}

This work represents: (1) a comprehensive fluid dynamic analysis to understand the fluid behavior in orbiting Petri-dishes, and (2) a novel study in analyzing in-vitro endothelial cellular responses under exposure of oscillatory fluidic shear stress. Computational fluid dynamics (CFD) was used to model oscillatory WSS in the dish, which provided complete spatial and temporal resolution of shear on the bottom surface of the dish. The computational WSS was compared and verified with previously reported experimental data.

Next, the fluid flow in orbiting Petri-dishes was characterized in terms of three dimensionless flow parameters: Stokes number, Froude number, and Slope ratio. The study characterized WSS contours and distribution between WSS components relative to each parameter's transition point.

In the final part of the study, the model was applied to determine the oscillatory shear effects on endothelial cellular responses such as proliferation and morphology. HUVEC cells in culture medium were exposed to oscillatory WSS by using an orbital shaker with specified orbital speeds. Shear in a dish was seen to be bi-directional, so a new hemodynamic parameter - Directional Oscillatory Shear Index (DOSI) was defined 
to account for the effects of directionality of shear on cellular responses in relation to principal axes. The results interestingly demonstrated that in addition to shear magnitude, direction of shear also significantly affected the cellular responses. Also the effects of directionality of shear on atherogenic gene expressions (E-Selectin, I-CAM, IL6) of HUVEC cells were studied in the presence of atherogenic regulators $\left(\mathrm{LTB}_{4}\right.$ and LPS).

Results showed that bi-directional shear increases those expressions, while unidirectional tangential WSS decreases those expressions. This may be one of the most important findings in understanding the cause of atherosclerotic plaque development. As a future continuation of this finding, one can model the in-vivo arterial blood flow to quantify the magnitude and directional effects on atherosclerotic plaque development.

\subsection{RECOMMENDATIONS:}

The following recommendations are proposed for continuation of this effort

i) Validation of the CFD model using Particle Image Velocimetry (PIV)

ii) Comparison of WSS effects on cellular responses in orbital dish to that in a flow chamber system that emulates DOSI and changing direction.

iii) An effort was made to understand the oscillatory WSS effects on in-vitro cellular responses. It is next recommended to continue this study on an in-vivo arterial flow system in order to understand the actual WSS effects on different inflammatory diseases.

iv) Determine the possibility of the existence of transition "regions" rather than just a single transition point (similar to how Reynolds number for flow in a 
pipe has distinct laminar and turbulent regions with a wide transition region in between). 


\section{REFERENCES}

1. McCabe WL, Smith JC, Harriott P. Unit Operations in Chemical engineering. New York: McGraw-Hill Book Co.; 1993.

2. Bird RB, Stewart WE, Lightfoot EN. Transport Phenomena: John Wiley \& Sons, Inc; 2002.

3. Ohashi T, Sato M. Remodeling of vascular endothelial cells exposed to fluid shear stress: experimental and numerical approach. Fluid Dynamics Research. 2005:40-59.

4. Levesque MJ, Nerem RM. The elongation and orientation of cultured endothelial cells in response to shear stress. J Biomech Eng. 1985;107(4):341-7.

5. Kondapalli J, Flozak AS, Albuquerque ML. Laminar shear stress differentially modulates gene expression of p120 catenin, Kaiso transcription factor, and vascular endothelial cadherin in human coronary artery endothelial cells. J Biol Chem. 2004;279(12):11417-24.

6. McKinney VZ, Rinker KD, Truskey GA. Normal and shear stresses influence the spatial distribution of intracellular adhesion molecule-1 expression in human umbilical vein endothelial cells exposed to sudden expansion flow. J Biomech. 2006;39(5):806-17. 7. Partridge J, Carlsen H, Enesa K, Chaudhury H, Zakkar M, Luong L, et al. Laminar shear stress acts as a switch to regulate divergent functions of NF-kappaB in endothelial cells. FASEB J. 2007;21(13):3553-61. 
8. Chotard-Ghodsnia R, Haddad O, Leyrat A, Drochon A, Verdier C, Duperray A. Morphological analysis of tumor cell/endothelial cell interactions under shear flow. J Biomech. 2007;40(2):335-44. PMCID: 1961634.

9. Sirois E, Charara J, Ruel J, Dussault JC, Gagnon P, Doillon CJ. Endothelial cells exposed to erythrocytes under shear stress: an in vitro study. Biomaterials. $1998 ; 19(21): 1925-34$.

10. Sato M, Ohshima N, Nerem RM. Viscoelastic properties of cultured porcine aortic endothelial cells exposed to shear stress. J Biomech. 1996;29(4):461-7.

11. Sato M, Nagayama K, Kataoka N, Sasaki M, Hane K. Local mechanical properties measured by atomic force microscopy for cultured bovine endothelial cells exposed to shear stress. J Biomech. 2000;33(1):127-35.

12. Zhao F, Chella R, Ma T. Effects of shear stress on 3-D human mesenchymal stem cell construct development in a perfusion bioreactor system: Experiments and hydrodynamic modeling. Biotechnol Bioeng. 2007;96(3):584-95.

13. Papadaki M, McIntire LV, Eskin SG. Effects of shear stress on the growth kinetics of human aortic smooth muscle cells in vitro. Biotechnol Bioeng. $1996 ; 50(5): 555-61$.

14. Nollert MU, Diamond SL, McIntire LV. Hydrodynamic shear stress and mass transport modulation of endothelial cell metabolism. Biotechnol Bioeng. 1991;38(6):588602.

15. Sakamoto N, Ohashi T, Sato M. Effects of shear stress on permiability of vascular endothelial monolayer cocultured with smooth muscle cells. JSME International Journal. 2004;47(4):992-9. 
16. Zeng Y, Qiao Y, Zhang Y, Liu X, Wang Y, Hu J. Effects of fluid shear stress on apoptosis of cultured human umbilical vein endothelial cells induced by LPS. Cell Biol Int. 2005;29(11):932-5.

17. Jin X, Iwasa S, Okada K, Ooi A, Mitsui K, Mitsumata M. Shear stress-induced collagen XII expression is associated with atherogenesis. Biochem Biophys Res Commun. 2003;308(1):152-8.

18. Kudo SY, R. Machida, K. Ikeda, M. Oka, K. Tanishita, K. Effects of long term shear stress exposure on calcium response and morphology of cultured endothelial cells. JSME International Journal. 2003;46(4):1226-33.

19. Fukushima A, Nagatsu A, Kaibara M, Oka K, Tanishita K. Measurement of surface topography of endothelial cell and wall shear stress distribution on the cell. JSME International Journal. 2001;44(4):972-81.

20. Nagayama K, Fujii M, Kudou S, Tanishita K. Behavior of individual endothelial cell under shear stress. Bioengineering Conference1995. p. 515-6.

21. Dewey CF, Jr. Effects of fluid flow on living vascular cells. J Biomech Eng. $1984 ; 106(1): 31-5$.

22. Hermann C, Zeiher AM, Dimmeler S. Shear stress inhibits H2O2-induced apoptosis of human endothelial cells by modulation of the glutathione redox cycle and nitric oxide synthase. Arterioscler Thromb Vasc Biol. 1997;17(12):3588-92.

23. Feugier P, Black RA, Hunt JA, How TV. Attachment, morphology and adherence of human endothelial cells to vascular prosthesis materials under the action of shear stress. Biomaterials. 2005;26(13):1457-66. 
24. Ley K, Lundgren E, Berger E, Arfors KE. Shear-dependent inhibition of granulocyte adhesion to cultured endothelium by dextran sulfate. Blood. 1989;73(5):1324-30.

25. Owatverot TB, Oswald SJ, Chen Y, Wille JJ, Yin FC. Effect of combined cyclic stretch and fluid shear stress on endothelial cell morphological responses. J Biomech Eng. 2005;127(3):374-82.

26. Langerquist KA, Suvatne J, Barakat AI, editors. Multi-directional floe chamber: Analysis of endothelial cell morphology dependence on different shear forces. Proceedings of the second joint EMBS/BMES conference; 2002; Houston, TX, USA.

27. Hsiai TK, Cho SK, Wong PK, Ing M, Navab M, Reddy S, et al., editors. BioMEMS sensors for real time shear stress on endothelial cell dynamics. Proceedings of the second joint EMBS/BMES conference; 2002.

28. Haritonnidis $\mathrm{JH}$. The measurement of shear stress Advances in fluid mechanics measurements: Springer-Verlag; 1989. p. 229-36.

29. Chiu JJ, Chen LJ, Chen CN, Lee PL, Lee CI. A model for studying the effect of shear stress on interactions between vascular endothelial cells and smooth muscle cells. J Biomech. 2004;37(4):531-9.

30. Inoguchi $\mathrm{H}$, Tanaka $\mathrm{T}$, Maehara $\mathrm{Y}$, Matsuda $\mathrm{T}$. The effect of gradually graded shear stress on the morphological integrity of a huvec-seeded compliant small-diameter vascular graft. Biomaterials. 2007;28(3):486-95.

31. Berson RE, Purcell MR, Sharp MK, editors. Fluid motion in an orbiting culture dish. World Cong Biomech; 2006 July 30 - Aug 4; Munich, Germany. 
32. Yun S, Dardik A, Haga M, Yamashita A, Yamaguchi S, Koh Y, et al. Transcription factor Spl phosphorylation induced by shear stress inhibits membrane type 1-matrix metalloproteinase expression in endothelium. $\mathrm{J}$ Biol Chem. 2002;277(38):34808-14.

33. Pearce MJ, McIntyre TM, Prescott SM, Zimmerman GA, Whatley RE. Shear stress activates cytosolic phospholipase A2 (cPLA2) and MAP kinase in human endothelial cells. Biochem Biophys Res Commun. 1996;218(2):500-4.

34. Kraiss LW, Weyrich AS, Alto NM, Dixon DA, Ennis TM, Modur V, et al. Fluid flow activates a regulator of translation, p70/p85 S6 kinase, in human endothelial cells. Am J Physiol Heart Circ Physiol. 2000;278(5):H1537-44.

35. Haga M, Yamashita A, Paszkowiak J, Sumpio BE, Dardik A. Oscillatory shear stress increases smooth muscle cell proliferation and Akt phosphorylation. J Vasc Surg. 2003;37(6):1277-84.

36. Dardik A, Chen L, Frattini J, Asada H, Aziz F, Kudo FA, et al. Differential effects of orbital and laminar shear stress on endothelial cells. J Vasc Surg. 2005;41(5):869-80.

37. Anderson JDJ. Computational Fluid Dynamics - A Engineering Tool ? Numerical laboratory computational methods in Fluid Dynamics, ASME. 1976:1-12.

38. Chung TJ. Computational Fluid Dynamics. Cambridge, United Kingdom: Cambridge University Press; 2002.

39. Graves RA. Computational Fluid Dynamics - The coming revolution. Astronautics and Aeronautics. 1982;20(3):20-8.

40. Kopal Z. Tables of supersonic flow around cones. Cambridge: Massachusetts Institute of technology; 1947. 
41. Taylor GI, Maccoll JW, editors. The air pressure on a cone moving at high speed. Proceedings of the Royal Society (A); 1933.

42. Fay JA, Riddell FR. Theory of stagnation point heat transfer in dissotiated air Journal of Aeronautical Science. 1958;25(2):73-85.

43. Blottner FG. Chemical nonequilibrium boundary layer. AIAA. 1964;2(2):232-9.

44. Blottner FG. Nonequilibrium laminar boundary layer flow of ionized air. AIAA. 1964;2(11):1921-7.

45. Hall HG, Eschenroeder AQ, Marrone PV. Blunt-nose inviscid airflows with coupled nonequilibrium processes. Journal of the aerospace science. 1962;19(9):1038-51.

46. Anderson JD, Dick E, Degrez G, Grundmann R, Degroote J, Vierendeels J. Computational Fluid Dynamics - an Introduction. 3rd ed. Wendt PDJF, editor: Springer; 2009.

47. ANSYS I. FLUENT 6.2.5. Canonsburg, PA2006.

48. FLUENT I. FLUENT user guide. Lebanon, NH2007.

49. Tu J, Yeoh GH, Liu C. Computational Fluid Dynamics : A computational approach. 1st ed. Burlington, MA: Elsevier; 2008.

50. Bhaskaran R, Collins L. Introduction to CFD basics: Cornell University; 2002.

51. Gerber S, Oevermann M, Behrendt F, editors. An Euler-Lagrange modeling approach for the simulation of wood gasification in fluidized beds. Proceedings of European combustion meeting; 2009.

52. Sakurai A NA, Yamaguchi T, Masuda M, Fujiwara K. A computational fluid mechanical study of flow over cultured endothelial cells. Advances in Bioengineering. $1991 ; 20: 289-302$. 
53. Satcher RL, Jr., Bussolari SR, Gimbrone MA, Jr., Dewey CF, Jr. The distribution of fluid forces on model arterial endothelium using computational fluid dynamics. J Biomech Eng. 1992;114(3):309-16.

54. Buschmann MH, Dieterich P, Adams NA, Schnittler HJ. Analysis of flow in a cone-and-plate apparatus with respect to spatial and temporal effects on endothelial cells. Biotechnol Bioeng. 2005;89(5):493-502.

55. Marshall I, Zhao S, Papathanasopoulou P, Hoskins P, Xu Y. MRI and CFD studies of pulsatile flow in healthy and stenosed carotid bifurcation models. J Biomech. 2004;37(5):679-87.

56. Yamaguchi T, Hanai S. Measured wall shear stress distribution pattern upstream and downstream of a unilateral stenosis by an electrochemical method. Biorheology. $1987 ; 24(6): 753-62$.

57. Yamaguchi T, Yamamoto Y, Liu H. Computational mechanical model studies on the spontaneous emergent morphogenesis of the cultured endothelial cells. J Biomech. $2000 ; 33(1): 115-26$

58. Weston SJ, Wood NB, Tabor G, Gosman AD, Firmin DN. Combined MRI and CFD analysis of fully developed steady and pulsatile laminar flow through a bend. $\mathbf{J}$ Magn Reson Imaging. 1998;8(5):1158-71.

59. Banerjee RK, Back LH, Back MR, Cho YI. Physiological flow simulation in residual human stenoses after coronary angioplasty. J Biomech Eng. 2000;122(4):310-20. 60. Papathanasopoulou P, Zhao S, Kohler U, Robertson MB, Long Q, Hoskins P, et al. MRI measurement of time-resolved wall shear stress vectors in a carotid bifurcation 
model, and comparison with CFD predictions. J Magn Reson Imaging. 2003;17(2):15362.

61. Morbiducci U, Ponzini R, Grigioni M, Redaelli A. Helical flow as fluid dynamic signature for atherogenesis risk in aortocoronary bypass. A numeric study. J Biomech. $2007 ; 40(3): 519-34$.

62. Brown A, Meenam B, editors. Investigating the effects of fluid shear forces on cellular responses to profiled surfaces in-vitro: A computational and experimental investigation2007; Lyon, France.

63. Berthier B, Bouzerar R, Legallais C. Blood flow patterns in an anatomically realistic coronary vessel: influence of three different reconstruction methods. $\mathbf{J}$ Biomech. 2002;35(10):1347-56.

64. Jung J, Hassanein A. Three-phase CFD analytical modeling of blood flow. Med Eng Phys. 2008;30(1):91-103.

65. Tokuda Y, Song MH, Ueda Y, Usui A, Akita T, Yoneyama S, et al. Threedimensional numerical simulation of blood flow in the aortic arch during cardiopulmonary bypass. Eur J Cardiothorac Surg. 2008;33(2):164-7.

66. Dong X, Wang K. Prediction and simulation on the shear stress and mass transfer in perfused bioreactor I : Circular bioreactor. Chinese Control and Decision Conference2008. p. $4237-42$.

67. Nanduri JR, F.A. P-R, Celik I. CFD mesh generation for biological flows: Geometry reconstruction using disgnostic images. Computers \& Fluids. 2009;38:1026-32 
68. Van Tricht I, De Wachter D, Tordoir J, Verdonck P. Comparison of the hemodynamics in $6 \mathrm{~mm}$ and 4-7 $\mathrm{mm}$ hemodialysis grafts by means of CFD. J Biomech. $2006 ; 39(2): 226-36$

69. Dehlaghi V, Shadpoor MT, Najarian S. Analysis of wall shear stress in stented coronary artery using 3D computational fluid dynamics modeling. Journal of Materials Processing Technology. 2008;197:I74-I81.

70. Fukushima SG, H. Tanishita, K. . Shear stress distribution on the surface endothelial cells during flow-induced morphological remodeling. JSME International Journal. 2003;46(4):1275-83.

71. Smith S, Austin S, Wesson GD, Moore CA. Calculation of wall shear stress in left coronary artery bifurcation for pulsatile flow using two-dimensional computational fluid dynamics. Conf Proc IEEE Eng Med Biol Soc. 2006;1:871-4.

72. Rance $\mathrm{P}$, Warren $\mathrm{N}$, editors. The inception movement of course material is oacillatory flow. Proceedings of the 11 th conference in Costal Engineering; 1968.

73. Sugaya Y, Ihara K, Masuda Y, Ohtsubo E, Maki H. Hyper-processive and slower DNA chain elongation catalysed by DNA polymerase III holoenzyme purified from the dnaE173 mutator mutant of Escherichia coli. Genes Cells. 2002;7(4):385-99.

74. Li YS, Haga JH, Chien S. Molecular basis of the effects of shear stress on vascular endothelial cells. J Biomech. 2005;38(10):1949-71.

75. Milovanova T, Manevich Y, Haddad A, Chatterjee S, Moore JS, Fisher AB. Endothelial cell proliferation associated with abrupt reduction in shear stress is dependent on reactive oxygen species. Antioxid Redox Signal. 2004;6(2):245-58. 
76. Secomb TW, Pries AR. Mechanics of shear stress transmission to endothelial cells in blood vessels lined with an endothelial surface layer. Bioengineering Conference2001. p. 389-90.

77. Slater JH, Jain S, Coger RN, Lee CY. The effects of shear stress on endothelial cells at hypothermic temperatures. ASME International Mechanical Engineering Congress \& Exposition2002. p. 219-25.

78. Sato M, Nagayam K, Kataoka N, Sasaki M, Hane K. Shear stress induced changes in endothelial cell stiffness measured bu atomic force microscopy. Bioengineering Conference1999. p. 343-4.

79. Fernandez P, Daculsi R, Remy-Zolghadri M, Bareille R, Bordenave L. Endothelial cells cultured on engineered vascular grafts are able to transduce shear stress. Tissue Eng. 2006;12(1):1-7.

80. Zhao S, Suciu A, Moore JE, Ziegler T, Burki E, Meister JJ, et al. Morphological effects of combined fluid shear stress and circumferential cyclic stretch on cultured vascular endothelial cells. Bioengineering Conference1995. p. 567-8.

81. Levesque MJ, Sprague EA, Scwartz CJ, Nerem RM. The influence of shear stress on cultured vascular endothelial cells: The stress response of an anchorage dependent mammalian cell. Biotechnology Progress. 1989;5(1):1-8.

82. Kudo S, Ikezawa K, Matsumara S, Ikeda M, Oka K, Tanishita K. Effects of shear stress on albumin uptake into cultured endothelial cells. Advances in Bioengineering. 1996;33:209-10. 
83. Tanishita K, Nagayama K, Fujii M, Kudo S. Empirical study on grouping behavior of individual endothelial cells under shear stress. JSME International Journal. 1999;42(3):715-9.

84. Hughes SK, Wacker BK, Kaneda MM, Elbert DL. Fluid shear stress modulates cell migration induced by sphingosine 1-phosphate and vascular endothelial growth factor. Ann Biomed Eng. 2005;33(8):1003-14.

85. Martin SF, Alevriadou BR, editors. Oxidative stress and lipid peroxidation due to endothelial cell exposure to fluid shear stress. Second Joint EMBS/BMES Conference; 2002.

86. Tang D, Yang C, Liu SQ, editors. Shear stress distribution on the membrane of endothelial cells using 3-D computational modeling with fluid structure interactions. Proceedings of second joint EMBS/BMES conference; 2002.

87. Hsiai TK, Cho SK, Wong PK, Ing M, Salazar A, Sevanian A, et al. Monocyte recruitment to endothelial cells in response to oscillatory shear stress. FASEB J. $2003 ; 17(12): 1648-57$.

88. Chappell DC, Varner SE, Nerem RM, Medford RM, Alexander RW. Oscillatory shear stress stimulates adhesion molecule expression in cultured human endothelium. Circ Res. 1998;82(5):532-9.

89. Ku DN, Giddens DP, Zarins CK, Glagov S. Pulsatile flow and atherosclerosis in the human carotid bifurcation. Positive correlation between plaque location and low oscillating shear stress. Arteriosclerosis. 1985;5(3):293-302. 
90. Ding Z, Wang K, Li J, Cong X. Flow field and oscillatory shear stress in a tuningfork-shaped model of the average human carotid bifurcation. $\mathbf{J}$ Biomech. $2001 ; 34(12): 1555-62$.

91. Hwang J, Saha A, Boo YC, Sorescu GP, McNally JS, Holland SM, et al. Oscillatory shear stress stimulates endothelial production of $\mathrm{O} 2$ - from $\mathrm{p} 47 \mathrm{phox}-$ dependent $\mathrm{NAD}(\mathrm{P}) \mathrm{H}$ oxidases, leading to monocyte adhesion. $J$ Biol Chem. $2003 ; 278(47): 47291-8$

92. Han Y, Ganatos P, Weinbaum S. Transmission of steady and oscillatory fluid shear stress across epithelial and endothelial surface structures. Physics of Fluid. $2005 ; 17(031508): 1-13$.

93. Schmidt SM, McCready MJ, Ostafin AE. Effect of oscillating fluid shear on solute transport in cortical bone. J Biomech. 2005;38(12):2337-43.

94. Hsiai TK, Cho SK, Honda HM, Hama S, Navab M, Demer LL, et al. Endothelial cell dynamics under pulsating flows: significance of high versus low shear stress slew rates (d(tau)/dt). Ann Biomed Eng. 2002;30(5):646-56.

95. Davies PF, Remuzzi A, Gordon EJ, Dewey CF, Jr., Gimbrone MA, Jr. Turbulent fluid shear stress induces vascular endothelial cell turnover in vitro. Proc Natl Acad Sci U S A. 1986;83(7):2114-7. PMCID: 323241.

96. He $\mathrm{X}, \mathrm{Ku} \mathrm{DN}$. Pulsatile flow in the human left coronary artery bifurcation: average conditions. J Biomech Eng. 1996;118(1):74-82.

97. Milovanova T, Chatterjee S, Manevich Y, Kotelnikova I, Debolt K, Madesh M, et al. Lung endothelial cell proliferation with decreased shear stress is mediated by reactive oxygen species. Am J Physiol Cell Physiol. 2006;290(1):C66-76. 
98. Frangos JA, McIntire LV, Eskin SG. Shear stress induced stimulation of mammalian cell metabolism. Biotechnol Bioeng. 1988;32(8):1053-60.

99. Fisher AB, Chien S, Barakat AI, Nerem RM. Endothelial cellular response to altered shear stress. Am J Physiol Lung Cell Mol Physiol. 2001;281(3):L529-33.

100. Himburg HA, Dowd SE, Friedman MH. Frequency-dependent response of the vascular endothelium to pulsatile shear stress. Am J Physiol Heart Circ Physiol. 2007;293(1):H645-53.

101. Hwang J, Ing MH, Salazar A, Lassegue B, Griendling K, Navab M, et al. Pulsatile versus oscillatory shear stress regulates NADPH oxidase subunit expression: implication for native LDL oxidation. Circ Res. 2003;93(12):1225-32.

102. Bacabac RG, Smit TH, Van Loon JJ, Doulabi BZ, Helder M, Klein-Nulend J. Bone cell responses to high-frequency vibration stress: does the nucleus oscillate within the cytoplasm? FASEB J. 2006;20(7):858-64.

103. Berson RE, Purcell MR, Sharp MK. Computationally determined shear on cells grown in orbiting culture dishes. Adv Exp Med Biol. 2008;614:189-98.

104. Nerem RM, Levesque MJ, Cornhill JF. Vascular endothelial morphology as an indicator of the pattern of blood flow. J Biomech Eng. 1981;103(3):172-6.

105. Dobson AJ. An introduction to generalized linear models: Chapman \& Hall 1990.

106. Hastie TJaP, D. . Generalized linear models, Chapter 6 of statistical models: Wordsworth \& Brooks/Cole; 1992.

107. McCullagh PN, J.A. Generalized Linear Models. London: Chapman \& Hill; 1089. 
108. Fisher NL, A. Regression models for an angular response. Biometrics. $1992 ; 48: 665-77$.

109. Fisher NL, T.E. . Estimating the common mean direction of several circular or spherical; distributions with different dispersions. Biometrika. 1983;70:9.

110. Green P. Iteratively reweighted least squares for maximum likelyhood estimation and some robust and resistant alternatives. Journal of Royal Statistical Society. $1984 ; 46(\mathrm{~B}): 44$.

111. Jammalamadaka SS, A. Topics in circular statistics. World Scientific Press2001. 112. Sharma YJ, S, editor. Circular regression in statistical science and data analysis. Proceeding of the third Pacific Area Statistical Conference; 1993; Utrecht, Netherlands.

113. Asada H, Paszkowiak J, Teso D, Alvi K, Thorisson A, Frattini JC, et al. Sustained orbital shear stress stimulates smooth muscle cell proliferation via the extracellular signal-regulated protein kinase 1/2 pathway. J Vasc Surg. 2005;42(4):772-80.

114. Malek AM, Izumo S. Mechanism of endothelial cell shape change and cytoskeletal remodeling in response to fluid shear stress. J Cell Sci. 1996;109 ( Pt 4):71326.

115. Chien S, Shyy JY. Effects of hemodynamic forces on gene expression and signal transduction in endothelial cells. Biol Bull. 1998;194(3):390-1; discussion 2-3.

116. Vara DS, Punshon G, Sales KM, Hamilton G, Seifalian AM. The effect of shear stress on human endothelial cells seeded on cylindrical viscoelastic conduits: an investigation of gene expression. Biotechnol Appl Biochem. 2006;45(Pt 3):119-30.

117. Lin K, Shyy JY, Chien S, editors. Effects of shear stress on tissue factor gene expression in endothelial cells. Joint BMES/EMBS Conference; 1999. 
118. DePaola $N$. The effect of shear stress gradient on endothelial cell proliferation and monolayer integrity. Advances in Bioengineering. 1994;28:321-2.

119. Guild JB, Chromiak AB, Sprague RM, Nerem RM, editors. Dyanamic MCP-1 mRNA responses of human aortic endothelial cells (HAEC) to step changes in laminar shear stress. Proceedings of the first joint EMBS/BMES conference; 1999.

120. Chachisvilis M, Zhang YL, Frangos JA. G protein-coupled receptors sense fluid shear stress in endothelial cells. Proc Natl Acad Sci U S A. 2006;103(42):15463-8. PMCID: 1622845.

121. Libby P. Inflammation in atherosclerosis. Nature. 2002;420(6917):868-74.

122. Cunningham KS, Gotlieb AI. The role of shear stress in the pathogenesis of atherosclerosis. Lab Invest. 2005;85(1):9-23.

123. Ferrero-Miliani L, Nielsen $\mathrm{OH}$, Andersen PS, Girardin SE. Chronic inflammation: importance of NOD2 and NALP3 in interleukin-1beta generation. Clin Exp Immunol. 2007;147(2):227-35. PMCID: 1810472.

124. Ridger V, Krams R, Carpi A, Evans PC. Hemodynamic parameters regulating vascular inflammation and atherosclerosis: a brief update. Biomed Pharmacother. 2008;62(8):536-40.

125. Ross R. Atherosclerosis is an inflammatory disease. Am Heart J. 1999;138(5 Pt 2):S419-20.

126. Traub O, Berk BC. Laminar shear stress: mechanisms by which endothelial cells transduce an atheroprotective force. Arterioscler Thromb Vasc Biol. 1998;18(5):677-85. 
127. Sumagin R, Lomakina E, Sarelius IH. Leukocyte-endothelial cell interactions are linked to vascular permeability via ICAM-1-mediated signaling. Am J Physiol Heart Circ Physiol. 2008;295(3):H969-H77. PMCID: 2544502.

128. Nakashima Y, Raines EW, Plump AS, Breslow JL, Ross R. Upregulation of VCAM-1 and ICAM-1 at atherosclerosis-prone sites on the endothelium in the ApoEdeficient mouse. Arterioscler Thromb Vasc Biol. 1998;18(5):842-51.

129. Bourdillon MC, Poston RN, Covacho C, Chignier E, Bricca G, McGregor JL. ICAM-1 deficiency reduces atherosclerotic lesions in double-knockout mice (ApoE(-/)/ICAM-1(-/-)) fed a fat or a chow diet. Arterioscler Thromb Vasc Biol. $2000 ; 20(12): 2630-5$.

130. Frank PG, Lisanti MP. ICAM-1: role in inflammation and in the regulation of vascular permeability. Am J Physiol Heart Circ Physiol. 2008;295(3):H926-H7. PMCID: 2544488 .

131. Schieffer B, Selle T, Hilfiker A, Hilfiker-Kleiner D, Grote K, Tietge UJ, et al. Impact of interleukin-6 on plaque development and morphology in experimental atherosclerosis. Circulation. 2004; 1 10(22):3493-500.

132. Sukovich DA, Kauser K, Shirley FD, DelVecchio V, Halks-Miller M, Rubanyi GM. Expression of interleukin-6 in atherosclerotic lesions of male ApoE-knockout mice: inhibition by 17beta-estradiol. Arterioscler Thromb Vasc Biol. 1998;18(9):1498-505.

133. Steeber DA, Campbell MA, Basit A, Ley K, Tedder TF. Optimal selectinmediated rolling of leukocytes during inflammation in vivo requires intercellular adhesion molecule-1 expression. Proc Natl Acad Sci U S A. 1998;95(13):7562-7. PMCID: 22683. 
134. Galkina E, Ley K. Vascular adhesion molecules in atherosclerosis. Arterioscler Thromb Vasc Biol. 2007;27(11):2292-301.

135. Smith CW. Possible steps involved in the transition to stationary adhesion of rolling neutrophils: a brief review. Microcirculation. 2000;7(6 Pt 1):385-94.

136. Springer TA. Traffic signals for lymphocyte recirculation and leukocyte emigration: the multistep paradigm. Cell. 1994;76(2):301-14.

137. Schuett H, Luchtefeld M, Grothusen C, Grote K, Schieffer B. How much is too much? Interleukin-6 and its signalling in atherosclerosis. Thromb Haemost. 2009;102(2):215-22.

138. Nagel T, Resnick N, Atkinson WJ, Dewey CF, Jr., Gimbrone MA, Jr. Shear stress selectively upregulates intercellular adhesion molecule- 1 expression in cultured human vascular endothelial cells. J Clin Invest. 1994;94(2):885-91. PMCID: 296171.

139. Papadaki M, Eskin SG. Effects of fluid shear stress on gene regulation of vascular cells. Biotechnol Prog. 1997;13(3):209-21.

140. Chiu JJ, Lee PL, Chen CN, Lee CI, Chang SF, Chen LJ, et al. Shear stress increases ICAM-1 and decreases VCAM-1 and E-selectin expressions induced by tumor necrosis factor-[alpha] in endothelial cells. Arterioscler Thromb Vasc Biol. 2004;24(1):73-9.

141. Samuelsson B, Dahlen SE, Lindgren JA, Rouzer CA, Serhan CN. Leukotrienes and lipoxins: structures, biosynthesis, and biological effects. Science. 1987;237(4819):1171-6.

142. Dahlen SE. Leukotriene receptors. Clin Rev Allergy Immunol. 1999;17(1-2):17991. 
143. Dahlen SE. Treatment of asthma with antileukotrienes: first line or last resort therapy? Eur J Pharmacol. 2006;533(1-3):40-56.

144. Back M, Hansson GK. Leukotriene receptors in atherosclerosis. Ann Med. 2006;38(7):493-502.

145. Back M. Studies of receptors and modulatory mechanisms in functional responses to cysteinyl-leukotrienes in smooth muscle. Acta Physiol Scand Suppl. 2002;648:1-55.

146. Dahlen SE, Hedqvist P, Hammarstrom S, Samuelsson B. Leukotrienes are potent constrictors of human bronchi. Nature. 1980;288(5790):484-6.

147. Mathis SP, Jala VR, Lee DM, Haribabu B. Nonredundant roles for leukotriene B4 receptors BLT1 and BLT2 in inflammatory arthritis. J Immunol. 2010;185(5):3049-56.

148. Aiello RJ, Brees D, Bourassa PA, Royer L, Lindsey S, Coskran T, et al. Increased atherosclerosis in hyperlipidemic mice with inactivation of ABCAl in macrophages. Arterioscler Thromb Vasc Biol. 2002;22(4):630-7.

149. Mehrabian M, Allayee H, Wong J, Shi W, Wang XP, Shaposhnik Z, et al. Identification of 5-lipoxygenase as a major gene contributing to atherosclerosis susceptibility in mice. Circ Res. 2002;91(2):120-6.

150. Mehrabian M, Wong J, Wang X, Jiang Z, Shi W, Fogelman AM, et al. Genetic locus in mice that blocks development of atherosclerosis despite extreme hyperlipidemia. Circ Res. 2001;89(2):125-30.

151. Jala VR, Haribabu B. Leukotrienes and atherosclerosis: new roles for old mediators. Trends Immunol. 2004;25(6):315-22. 
152. Fujihara M, Muroi M, Tanamoto K, Suzuki T, Azuma H, Ikeda H. Molecular mechanisms of macrophage activation and deactivation by lipopolysaccharide: roles of the receptor complex. Pharmacol Ther. 2003;100(2):171-94.

153. VanderLaan PA, Reardon CA, Getz GS. Site specificity of atherosclerosis: siteselective responses to atherosclerotic modulators. Arterioscler Thromb Vasc Biol. 2004;24(1):12-22.

154. Walsh M, McGloughlin T, Liepsch DW, O'Brien T, Morris L, Ansari AR. On using experimentally estimated wall shear stresses to validate numerically predicted results. Proc Inst Mech Eng H. 2003;217(2):77-90.

155. Ni CW, Qiu H, Rezvan A, Kwon K, Nam D, Son DJ, et al. Discovery of novel mechanosensitive genes in vivo using mouse carotid artery endothelium exposed to disturbed flow. Blood. 2010.

156. Dahl A, Sultan M, Jung A, Schwartz R, Lange M, Steinwand M, et al. Quantitative PCR based expression analysis on a nanoliter scale using polymer nano-well chips. Biomed Microdevices. 2007;9(3):307-14.

157. Venables WNR, B.D. Modern Applied Statistics with S. New York: Springer; 2002.

158. Sawa Y, Ueki T, Hata M, Iwasawa K, Tsuruga E, Kojima H, et al. LPS-induced IL-6, IL-8, VCAM-1, and ICAM-1 expression in human lymphatic endothelium. J Histochem Cytochem. 2008;56(2):97-109. PMCID: 2324174.

159. Chakraborty A, Chakraborty S, Jala VR, Sharp MK, Haribabu B, Berson RE. Effects of Bidirectional Oscillatory Shear Stress on Endothelial Cell Proliferation and Morphology. Biotechnology \& Bioengineering. 2010:1-15. 
160. Tong WG, Ding XZ, Hennig R, Witt RC, Standop J, Pour PM, et al. Leukotriene B4 receptor antagonist LY293111 inhibits proliferation and induces apoptosis in human pancreatic cancer cells. Clin Cancer Res. 2002;8(10):3232-42.

161. Wada K, Arita M, Nakajima A, Katayama K, Kudo C, Kamisaki Y, et al. Leukotriene B4 and lipoxin A4 are regulatory signals for neural stem cell proliferation and differentiation. FASEB J. 2006;20(11):1785-92.

162. Woo CH, You HJ, Cho SH, Eom YW, Chun JS, Yoo YJ, et al. Leukotriene B(4) stimulates Rac-ERK cascade to generate reactive oxygen species that mediates chemotaxis. J Biol Chem. 2002;277(10):8572-8.

163. Atluru D, Goodwin JS. Leukotriene B4 causes proliferation of interleukin 2dependent $\mathrm{T}$ cells in the presence of suboptimal levels of interleukin 2. Cell Immunol. $1986 ; 99(2): 444-52$.

164. Cheng J, Yuan J, Zheng J. [The effect of Lipopolysacharide (LPS) on morphology and function of human umbilical endothelial cells (HUVECs)]. Zhonghua Shao Shang Za Zhi. 2001;17(3):155-8. 
APPENDIX - A

\section{COMPUTER PROGRAMS FOR FLUENT SIMULATIONS}

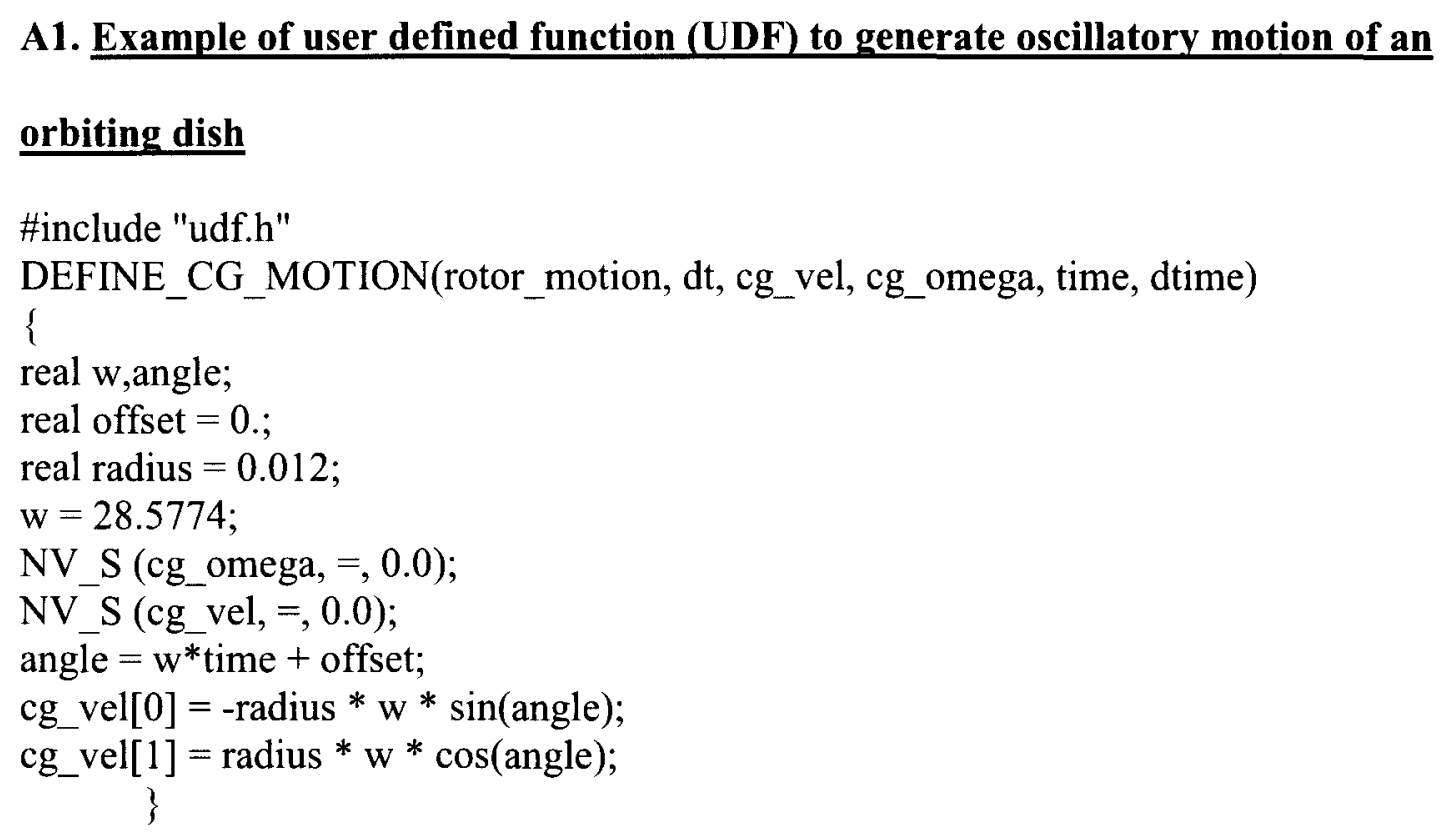




\section{A2. Example of a Batch file to submit the case to a node of Adelie}

\#!/bin/bash

\#PBS -q dualcore2

\#PBS -1 nodes=1:ppn=1

\#PBS -m e

\#PBS -M a0chak02@louisville.edu

INPUT_FILE=/homebackup/scrfluent/Amlan/dimensional_analysis/Set1/slope1/inputfile uss

OUTPUT_FILE=/homebackup/scrfluent/Amlan/dimensional_analysis/Set1/slope1/output file

$\mathrm{DIM}=3 \mathrm{~d}$

PROG="/apps/Fluent.Inc/bin/fluent "

PROGARGS="\$DIM -g -i \$INPUT_FILE"

echo Running on:

cat \$PBS NODEFILE

NPROCS ='wc $-1<$ \$PBS NODEFILE'

echo This job uses \$NPRŌCS processors

hostname

\$PROG \$PROGARGS $>$ \$OUTPUT FILE 2>\&1

\section{A3. Example of an Input File that is input to the Batch file}

$\mathrm{rc} /$ homebackup/scrfluent/Amlan/dimensional_analysis/Set1/slope1/slope 1.cas $\mathrm{rd} /$ homebackup/scrfluent/Amlan/dimensional_analysis/Set1/slope1/slope1.dat solve

d

859

50

wc /homebackup/scrfluent/Amlan/dimensional analysis/Set1/slope1/slopel final.cas wd /homebackup/scrfluent/Amlan/dimensional_analysis/Set1/slopel/slope1_final.dat quit exit yes 


\section{A4. Example of MATLAB code to determine the node values of tangential and}

\section{radial WSS at different radial locations on the bottom surface of a dish}

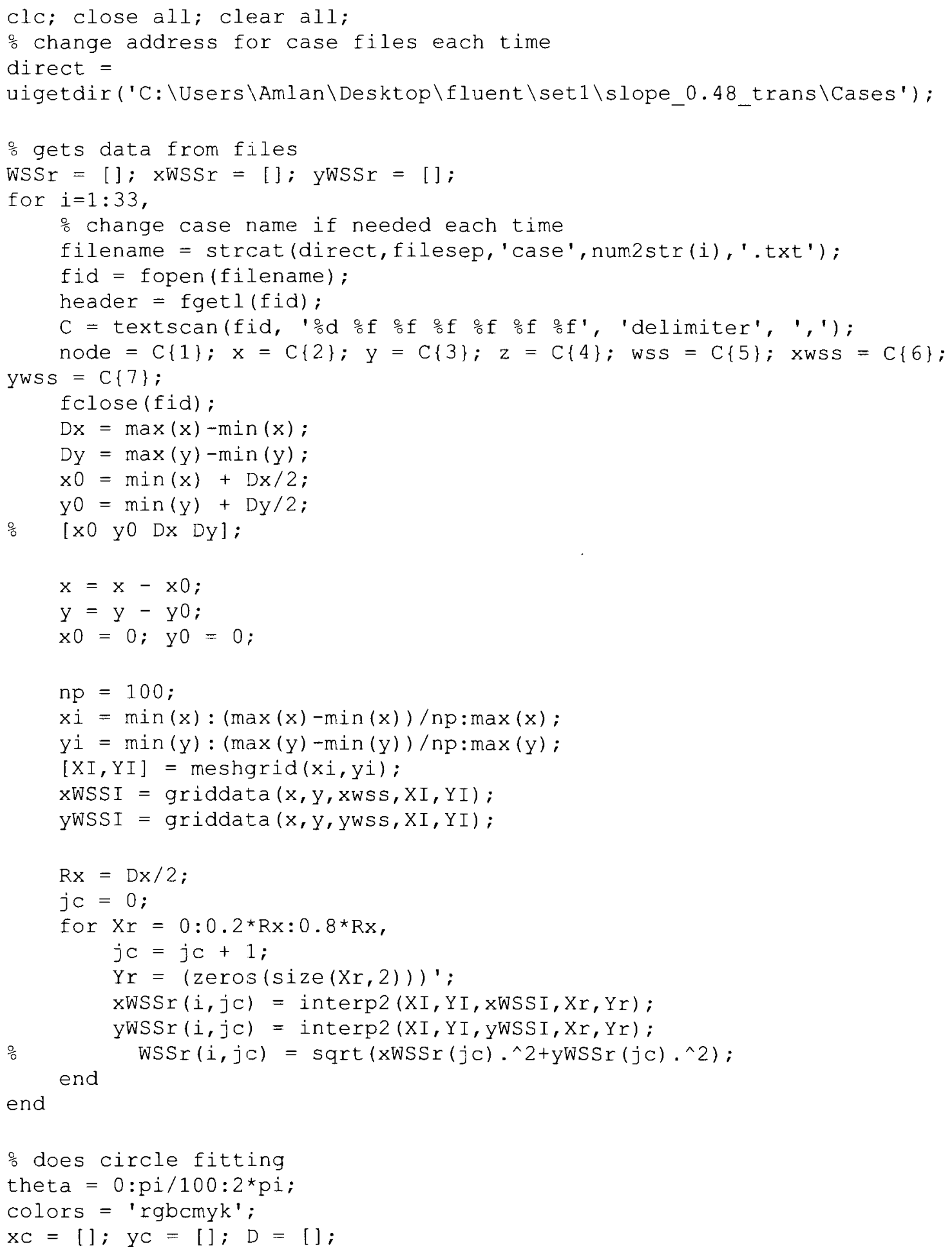




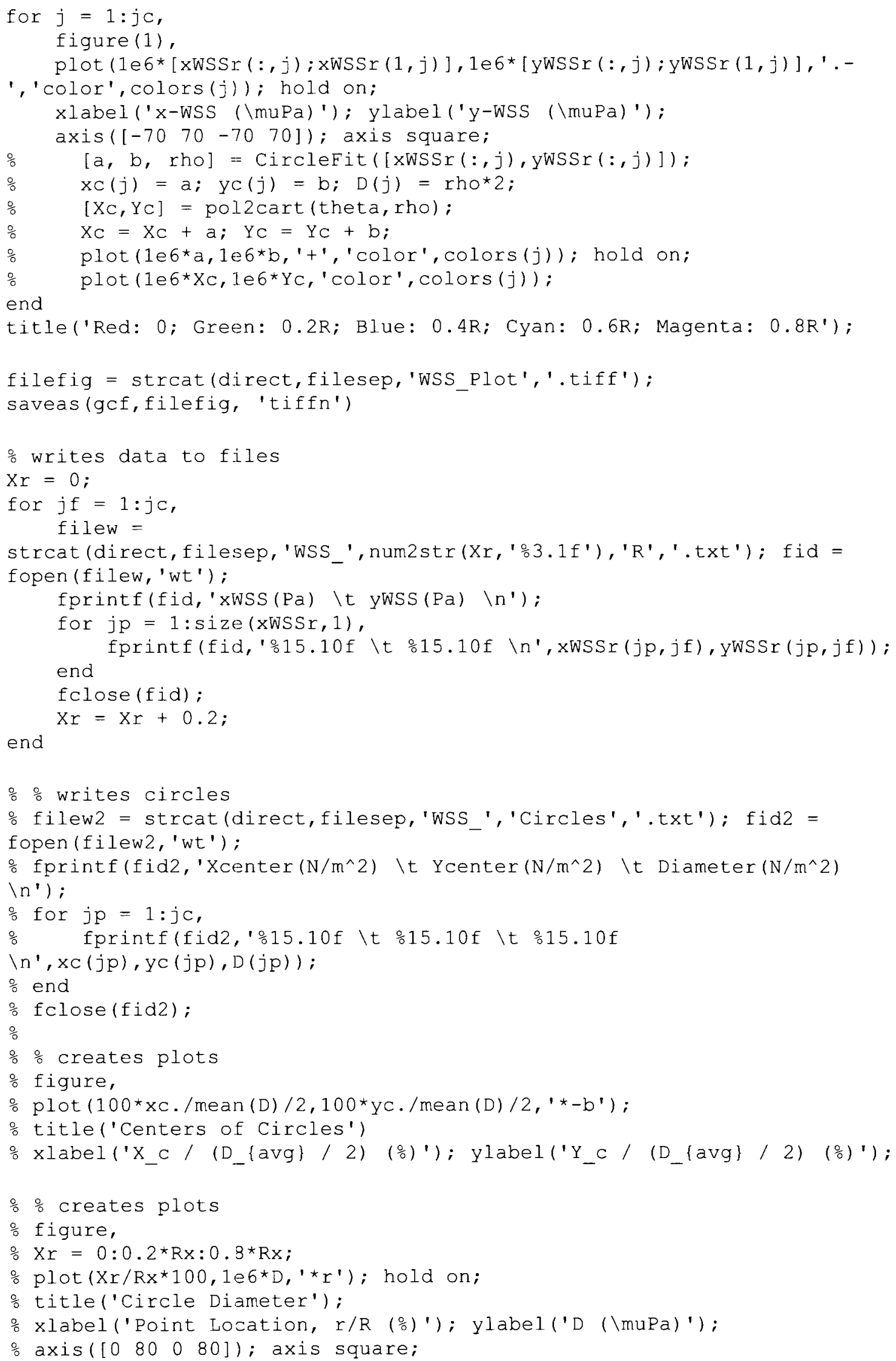




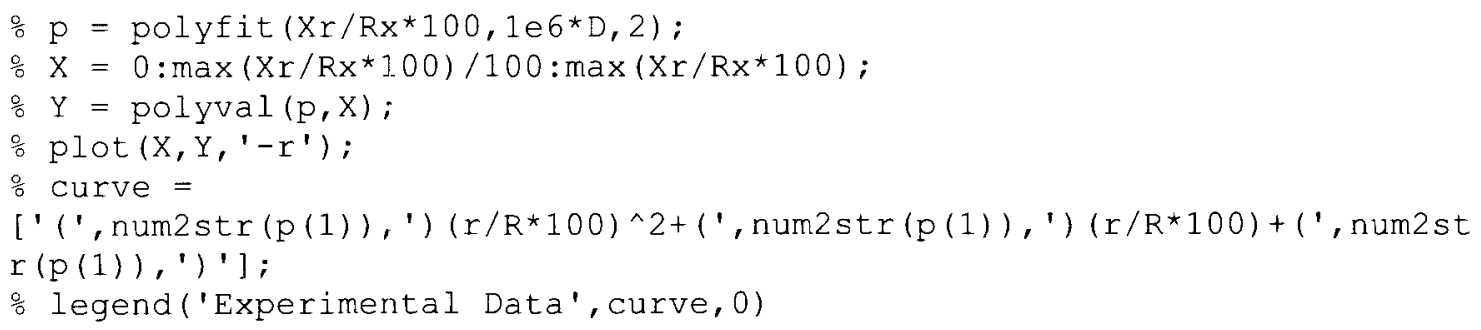




\section{APPENDIX - B}

\section{RADIAL AND TANGENTIAL WSS DATA FOR DIFFERENT COMBINATIONS}

\section{OF DIMENSIONLESS NUMBERS}

\section{B.1. WSS data as a function of Slope ratio at different combinations of constant}

\section{Froude Number and constant Stokes Number}

Table B1. Radial and tangential WSS values at Slope ratio of 0.1, Froude Number of 0.1

and Stokes Number of 1.

\begin{tabular}{|c|c|c|c|c|c|c|c|}
\hline \multicolumn{2}{|c|}{$20 \%$} & \multicolumn{2}{|c|}{$40 \%$} & \multicolumn{2}{|c|}{$60 \%$} & \multicolumn{2}{|c|}{$80 \%$} \\
\hline Radial & Tangential & Radial & Tangential & Radial & Tangential & Radial & Tangential \\
\hline $\mathrm{Pa}$ & $\mathrm{Pa}$ & $\mathrm{Pa}$ & $\mathrm{Pa}$ & $\mathrm{Pa}$ & $\mathbf{P a}$ & $\mathbf{P a}$ & $\mathrm{Pa}$ \\
\hline$-3.4095 \mathrm{E}-05$ & $-1.96741 \mathrm{E}-05$ & $-2.8 \mathrm{E}-05$ & $-1.6 \mathrm{E}-05$ & $-1.8 \mathrm{E}-05$ & $-1.2 \mathrm{E}-05$ & $-9.8 \mathrm{E}-06$ & $-1 E-05$ \\
\hline$-3.055 \mathrm{E}-05$ & $-2.24822 \mathrm{E}-05$ & $-2.5 \mathrm{E}-05$ & $-2.2 \mathrm{E}-05$ & $-1.8 \mathrm{E}-05$ & $-1.7 \mathrm{E}-05$ & $-9.3 \mathrm{E}-06$ & $-1.5 \mathrm{E}-05$ \\
\hline$-2.8509 \mathrm{E}-05$ & -0.000018969 & $-2.3 \mathrm{E}-05$ & $-2.1 \mathrm{E}-05$ & $-1.6 \mathrm{E}-05$ & $-1.7 \mathrm{E}-05$ & $-8.2 \mathrm{E}-06$ & $-1.5 \mathrm{E}-05$ \\
\hline$-2.3288 \mathrm{E}-05$ & $-2.34786 \mathrm{E}-05$ & $-1.7 \mathrm{E}-05$ & $-2.6 \mathrm{E}-05$ & $-1.3 \mathrm{E}-05$ & $-2.3 \mathrm{E}-05$ & $-5.7 \mathrm{E}-06$ & $-2 \mathrm{E}-05$ \\
\hline$-1.1674 \mathrm{E}-05$ & $-3.51235 \mathrm{E}-05$ & $-5.6 \mathrm{E}-06$ & $-3.8 \mathrm{E}-05$ & $-4.1 \mathrm{E}-06$ & $-3.5 \mathrm{E}-05$ & $-1.3 \mathrm{E}-06$ & $-3.1 \mathrm{E}-05$ \\
\hline$-1.0366 \mathrm{E}-06$ & $-3.91362 \mathrm{E}-05$ & $5.4 \mathrm{E}-06$ & $-4.1 \mathrm{E}-05$ & $4.06 \mathrm{E}-06$ & $-3.9 \mathrm{E}-05$ & $2.98 \mathrm{E}-06$ & $-3.5 \mathrm{E}-05$ \\
\hline $1.6595 \mathrm{E}-06$ & $-3.24136 \mathrm{E}-05$ & $9.63 \mathrm{E}-06$ & $-3.3 \mathrm{E}-05$ & $8.03 \mathrm{E}-06$ & $-3.2 \mathrm{E}-05$ & $5.36 \mathrm{E}-06$ & $-2.9 \mathrm{E}-05$ \\
\hline $4.8589 \mathrm{E}-06$ & $-2.58131 \mathrm{E}-05$ & $1.21 \mathrm{E}-05$ & $-2.5 \mathrm{E}-05$ & $8.78 \mathrm{E}-06$ & $-2.4 \mathrm{E}-05$ & $5.27 \mathrm{E}-06$ & $-2.1 \mathrm{E}-05$ \\
\hline 0.000013642 & $-2.49991 \mathrm{E}-05$ & $2.14 \mathrm{E}-05$ & $-2.5 \mathrm{E}-05$ & $1.72 \mathrm{E}-05$ & $-2.4 \mathrm{E}-05$ & $1.08 \mathrm{E}-05$ & $-2.2 \mathrm{E}-05$ \\
\hline $2.42501 \mathrm{E}-05$ & -0.000026129 & $3.2 \mathrm{E}-05$ & $-2.3 \mathrm{E}-05$ & $2.67 \mathrm{E}-05$ & $-2.2 \mathrm{E}-05$ & $1.66 \mathrm{E}-05$ & $-2 \mathrm{E}-05$ \\
\hline $2.72711 \mathrm{E}-05$ & $-2.36395 \mathrm{E}-05$ & $3.3 \mathrm{E}-05$ & $-1.8 \mathrm{E}-05$ & $2.79 \mathrm{E}-05$ & $-1.5 \mathrm{E}-05$ & $1.77 \mathrm{E}-05$ & $-1.3 \mathrm{E}-05$ \\
\hline $2.92746 \mathrm{E}-05$ & $-1.39336 \mathrm{E}-05$ & $3.14 \mathrm{E}-05$ & $-8.5 \mathrm{E}-06$ & $2.54 \mathrm{E}-05$ & $-3.8 \mathrm{E}-06$ & $1.74 \mathrm{E}-05$ & $-8.3 \mathrm{E}-07$ \\
\hline $3.42269 \mathrm{E}-05$ & $-1.6084 \mathrm{E}-06$ & $3.29 \mathrm{E}-05$ & $2.09 \mathrm{E}-06$ & $2.42 \mathrm{E}-05$ & $4.58 \mathrm{E}-06$ & $1.62 \mathrm{E}-05$ & $8.06 \mathrm{E}-06$ \\
\hline $3.72065 \mathrm{E}-05$ & $-1.2868 \mathrm{E}-06$ & $3.51 \mathrm{E}-05$ & $2.36 \mathrm{E}-06$ & $2.4 \mathrm{E}-05$ & $2.85 \mathrm{E}-06$ & $1.57 \mathrm{E}-05$ & $5.52 \mathrm{E}-06$ \\
\hline $3.86595 \mathrm{E}-05$ & $-5.1118 \mathrm{E}-06$ & $3.66 \mathrm{E}-05$ & $-2.2 \mathrm{E}-06$ & $2.52 \mathrm{E}-05$ & $-4.3 \mathrm{E}-06$ & $1.71 \mathrm{E}-05$ & $-3.5 \mathrm{E}-06$ \\
\hline $3.48984 \mathrm{E}-05$ & $8.1534 \mathrm{E}-06$ & $3.59 \mathrm{E}-05$ & $4.36 \mathrm{E}-06$ & $2.8 \mathrm{E}-05$ & $-4.2 \mathrm{E}-09$ & $2.24 \mathrm{E}-05$ & $-4.4 \mathrm{E}-06$ \\
\hline $2.91362 \mathrm{E}-05$ & $2.54333 \mathrm{E}-05$ & $3.59 \mathrm{E}-05$ & $1.36 \mathrm{E}-05$ & $2.99 \mathrm{E}-05$ & $6.67 \mathrm{E}-06$ & $2.69 \mathrm{E}-05$ & $-2.2 \mathrm{E}-08$ \\
\hline $2.71421 \mathrm{E}-05$ & $2.91528 \mathrm{E}-05$ & $3.76 \mathrm{E}-05$ & $1.45 \mathrm{E}-05$ & $3.39 \mathrm{E}-05$ & $1.02 \mathrm{E}-05$ & $3.24 \mathrm{E}-05$ & $-1.5 \mathrm{E}-06$ \\
\hline 0.00002718 & $2.65671 \mathrm{E}-05$ & $3.96 \mathrm{E}-05$ & $1.35 \mathrm{E}-05$ & $3.73 \mathrm{E}-05$ & $1.02 \mathrm{E}-05$ & $3.57 \mathrm{E}-05$ & $-2.9 \mathrm{E}-06$ \\
\hline $2.31082 \mathrm{E}-05$ & $2.86146 \mathrm{E}-05$ & $3.43 \mathrm{E}-05$ & $1.9 \mathrm{E}-05$ & $3.72 \mathrm{E}-05$ & $1.43 \mathrm{E}-05$ & $3.71 \mathrm{E}-05$ & $-2.1 \mathrm{E}-07$ \\
\hline $1.84308 \mathrm{E}-05$ & $3.28332 \mathrm{E}-05$ & $2.71 \mathrm{E}-05$ & $2.93 \mathrm{E}-05$ & $3.39 \mathrm{E}-05$ & $2.44 \mathrm{E}-05$ & $3.52 \mathrm{E}-05$ & $7.57 \mathrm{E}-06$ \\
\hline $1.82657 \mathrm{E}-05$ & $3.65255 \mathrm{E}-05$ & $2.38 \mathrm{E}-05$ & $3.73 \mathrm{E}-05$ & $3.08 \mathrm{E}-05$ & $3.27 \mathrm{E}-05$ & $3.2 \mathrm{E}-05$ & $1.54 \mathrm{E}-05$ \\
\hline $2.14862 \mathrm{E}-05$ & $3.86382 \mathrm{E}-05$ & $2.12 \mathrm{E}-05$ & $3.96 \mathrm{E}-05$ & $2.87 \mathrm{E}-05$ & $3.67 \mathrm{E}-05$ & $3 \mathrm{E}-05$ & $1.99 \mathrm{E}-05$ \\
\hline $1.31388 \mathrm{E}-05$ & $3.47353 \mathrm{E}-05$ & $1.36 \mathrm{E}-05$ & $3.74 \mathrm{E}-05$ & $2.32 \mathrm{E}-05$ & $3.83 \mathrm{E}-05$ & $2.61 \mathrm{E}-05$ & $2.38 \mathrm{E}-05$ \\
\hline$-7.5596 \mathrm{E}-06$ & $3.20852 \mathrm{E}-05$ & $1.23 \mathrm{E}-06$ & $3.98 \mathrm{E}-05$ & $1.37 \mathrm{E}-05$ & $4.15 \mathrm{E}-05$ & $1.71 \mathrm{E}-05$ & $2.99 \mathrm{E}-05$ \\
\hline$-2.624 \mathrm{E}-05$ & $2.69493 \mathrm{E}-05$ & $-4.5 \mathrm{E}-06$ & $4.57 \mathrm{E}-05$ & $1.48 \mathrm{E}-06$ & $4.16 \mathrm{E}-05$ & $6.18 \mathrm{E}-06$ & $3.42 \mathrm{E}-05$ \\
\hline$-3.5234 \mathrm{E}-05$ & 0.000019437 & $-1.7 \mathrm{E}-05$ & $3.87 \mathrm{E}-05$ & $-1.4 \mathrm{E}-05$ & $3.61 \mathrm{E}-05$ & $-1.9 \mathrm{E}-06$ & $3.45 \mathrm{E}-05$ \\
\hline$-3.3242 \mathrm{E}-05$ & 0.000013498 & $-2.4 \mathrm{E}-05$ & $2.64 \mathrm{E}-05$ & $-1.5 \mathrm{E}-05$ & $3.11 \mathrm{E}-05$ & $-5 \mathrm{E}-06$ & $3.13 \mathrm{E}-05$ \\
\hline$-2.9175 \mathrm{E}-05$ & $1.55634 \mathrm{E}-05$ & $-2.3 \mathrm{E}-05$ & $2.29 \mathrm{E}-05$ & $-1.6 \mathrm{E}-05$ & $1.94 \mathrm{E}-05$ & $-1.5 \mathrm{E}-05$ & $1.98 \mathrm{E}-05$ \\
\hline$-3.9157 \mathrm{E}-05$ & $8.4424 \mathrm{E}-06$ & $-2.7 \mathrm{E}-05$ & $1.87 \mathrm{E}-05$ & $-1.6 \mathrm{E}-05$ & $1.8 \mathrm{E}-05$ & $-1.5 \mathrm{E}-05$ & $1.4 \mathrm{E}-05$ \\
\hline$-4.2303 \mathrm{E}-05$ & 0.000003878 & $-3 \mathrm{E}-05$ & $9.83 \mathrm{E}-06$ & $-2.4 \mathrm{E}-05$ & $1.66 \mathrm{E}-05$ & $-1.3 \mathrm{E}-05$ & $1.37 \mathrm{E}-05$ \\
\hline$-3.7409 \mathrm{E}-05$ & $-6.5454 \mathrm{E}-06$ & $-3.1 \mathrm{E}-05$ & $-7.3 \mathrm{E}-06$ & $-2.2 \mathrm{E}-05$ & $3.49 \mathrm{E}-06$ & $-1.4 \mathrm{E}-05$ & $2.39 \mathrm{E}-06$ \\
\hline$-3.0497 \mathrm{E}-05$ & $-2.31608 \mathrm{E}-05$ & $-2.7 \mathrm{E}-05$ & $-1.5 \mathrm{E}-05$ & $-1.8 \mathrm{E}-05$ & $-1.3 \mathrm{E}-05$ & $-1.1 \mathrm{E}-05$ & $-1.1 \mathrm{E}-05$ \\
\hline
\end{tabular}


Table B2. Radial and tangential WSS values at Slope ratio of 0.48 , Froude Number of 0.1 and Stokes Number of 1 .

\begin{tabular}{|c|c|c|c|c|c|c|c|}
\hline \multicolumn{2}{|c|}{$20 \%$} & \multicolumn{2}{|c|}{$40 \%$} & \multicolumn{2}{|c|}{$60 \%$} & \multicolumn{2}{|c|}{$80 \%$} \\
\hline Radial & Tangential & Radial & Tangential & Radial & Tangential & Radial & Tangential \\
\hline $\mathrm{Pa}$ & $\mathrm{Pa}$ & $\mathrm{Pa}$ & $\mathrm{Pa}$ & $\mathrm{Pa}$ & $\mathrm{Pa}$ & $\mathrm{Pa}$ & $\mathrm{Pa}$ \\
\hline $2.80923 \mathrm{E}-05$ & 0.00013976 & $3.04 \mathrm{E}-05$ & 0.000129 & $2.44 \mathrm{E}-05$ & 0.000116 & $1.24 \mathrm{E}-05$ & $9.85 \mathrm{E}-05$ \\
\hline $3.9204 \mathrm{E}-06$ & 0.000142084 & $1.24 \mathrm{E}-05$ & 0.000132 & $1.31 \mathrm{E}-05$ & 0.000119 & $8.22 \mathrm{E}-06$ & 0.0001 \\
\hline$-2.0743 \mathrm{E}-05$ & 0.000140662 & $-6.7 E-06$ & 0.000133 & $1.49 \mathrm{E}-06$ & 0.000121 & $4.34 \mathrm{E}-06$ & 0.000103 \\
\hline-0.00004505 & 0.000134128 & $-2.7 \mathrm{E}-05$ & 0.000129 & $-1.3 \mathrm{E}-05$ & 0.00012 & $-2 \mathrm{E}-06$ & 0.000105 \\
\hline$-6.8466 \mathrm{E}-05$ & 0.00012384 & $-4.7 \mathrm{E}-05$ & 0.000123 & $-2.8 \mathrm{E}-05$ & 0.000118 & $-1 \mathrm{E}-05$ & 0.000106 \\
\hline$-9.0593 E-05$ & 0.000108502 & $-6.7 \mathrm{E}-05$ & 0.000112 & $-4.3 \mathrm{E}-05$ & 0.00011 & $-2.1 \mathrm{E}-05$ & 0.000102 \\
\hline-0.00011025 & $8.74855 \mathrm{E}-05$ & $-8.5 \mathrm{E}-05$ & $9.64 \mathrm{E}-05$ & $-5.9 \mathrm{E}-05$ & $9.8 \mathrm{E}-05$ & $-3.1 \mathrm{E}-05$ & $9.19 \mathrm{E}-05$ \\
\hline-0.00012663 & $6.34903 E-05$ & $-0.000 \mathrm{l}$ & $7.58 \mathrm{E}-05$ & $-7.3 \mathrm{E}-05$ & $8.07 \mathrm{E}-05$ & $-3.8 \mathrm{E}-05$ & $7.64 \mathrm{E}-05$ \\
\hline-0.00013899 & $3.52769 \mathrm{E}-05$ & -0.00012 & $5.06 \mathrm{E}-05$ & $-8.6 \mathrm{E}-05$ & $6.09 \mathrm{E}-05$ & $-5.3 \mathrm{E}-05$ & $8.11 \mathrm{E}-05$ \\
\hline-0.00014513 & $4.8677 \mathrm{E}-06$ & -0.00014 & $3.44 \mathrm{E}-05$ & -0.00015 & $8.94 \mathrm{E}-05$ & -0.00011 & 0.00014 \\
\hline-0.00014443 & $-2.37072 \mathrm{E}-05$ & -0.00019 & $2.39 \mathrm{E}-05$ & -0.00021 & $9.21 \mathrm{E}-05$ & -0.00016 & 0.000132 \\
\hline-0.00014264 & $-5.22881 \mathrm{E}-05$ & -0.00022 & $-5.8 \mathrm{E}-06$ & -0.00024 & $5.72 \mathrm{E}-05$ & -0.00018 & $8.29 \mathrm{E}-05$ \\
\hline-0.00014141 & $-8.38527 \mathrm{E}-05$ & -0.00023 & $-5 \mathrm{E}-05$ & -0.00025 & $-2.6 \mathrm{E}-06$ & -0.00019 & $1.56 \mathrm{E}-05$ \\
\hline-0.00014141 & $-8.38527 \mathrm{E}-05$ & -0.00023 & $-5 \mathrm{E}-05$ & -0.00025 & $-2.6 \mathrm{E}-06$ & -0.00019 & $1.56 \mathrm{E}-05$ \\
\hline-0.00010057 & -0.000148677 & -0.00018 & -0.00016 & -0.00021 & -0.00014 & -0.00015 & -0.00013 \\
\hline$-6.0694 \mathrm{E}-05$ & -0.000167992 & -0.00013 & -0.0002 & -0.00016 & -0.00021 & -0.00012 & -0.00019 \\
\hline$-1.5414 \mathrm{E}-05$ & -0.000172388 & $-5.5 \mathrm{E}-05$ & -0.00023 & $-8.9 \mathrm{E}-05$ & -0.00025 & $-7.2 \mathrm{E}-05$ & -0.00024 \\
\hline 0.00003001 & -0.000164706 & $2.84 \mathrm{E}-05$ & -0.00023 & $3.36 \mathrm{E}-06$ & -0.00027 & $-1.1 \mathrm{E}-05$ & -0.00028 \\
\hline $9.29256 \mathrm{E}-05$ & -0.000106435 & 0.000172 & -0.00013 & 0.000219 & -0.00016 & 0.000174 & -0.00021 \\
\hline 0.000108609 & $-7.63024 \mathrm{E}-05$ & 0.000193 & $-5.1 \mathrm{E}-05$ & 0.000279 & $-1.6 \mathrm{E}-05$ & 0.000271 & $-1.6 \mathrm{E}-05$ \\
\hline 0.000121837 & $-4.76244 \mathrm{E}-05$ & 0.000164 & $6.03 \mathrm{E}-06$ & 0.00024 & 0.000111 & 0.000258 & 0.000237 \\
\hline 0.000136376 & $-2.60194 \mathrm{E}-05$ & 0.000118 & $3.14 \mathrm{E}-05$ & 0.000122 & 0.000104 & 0.000114 & 0.000214 \\
\hline 0.000148264 & $-1.77178 \mathrm{E}-05$ & 0.000132 & $2.96 \mathrm{E}-05$ & 0.000103 & $7.68 \mathrm{E}-05$ & $6.12 \mathrm{E}-05$ & 0.000111 \\
\hline 0.000152815 & $8.1587 \mathrm{E}-06$ & 0.000133 & 4.64E-05 & 0.000104 & $7.48 \mathrm{E}-05$ & $6.14 \mathrm{E}-05$ & $8.93 \mathrm{E}-05$ \\
\hline 0.000147086 & $3.22241 \mathrm{E}-05$ & 0.000127 & $6.05 \mathrm{E}-05$ & $9.82 \mathrm{E}-05$ & 7.52E-05 & $5.85 \mathrm{E}-05$ & 7.74E-05 \\
\hline 0.000137755 & $5.63936 \mathrm{E}-05$ & 0.00012 & $7.08 \mathrm{E}-05$ & 8.94E-05 & $7.94 \mathrm{E}-05$ & $5.52 \mathrm{E}-05$ & 7.44E-05 \\
\hline 0.000126245 & $7.58057 \mathrm{E}-05$ & 0.000108 & $8.2 \mathrm{E}-05$ & $8.15 \mathrm{E}-05$ & $8.47 \mathrm{E}-05$ & $4.92 \mathrm{E}-05$ & $7.75 \mathrm{E}-05$ \\
\hline 0.0001109 & $9.38117 \mathrm{E}-05$ & $9.54 \mathrm{E}-05$ & $9.4 \mathrm{E}-05$ & $7.14 \mathrm{E}-05$ & $9.18 \mathrm{E}-05$ & $4.17 \mathrm{E}-05$ & 8.29E-05 \\
\hline 0.000092727 & 0.000110128 & $8.07 \mathrm{E}-05$ & 0.000106 & $5.98 \mathrm{E}-05$ & $9.92 \mathrm{E}-05$ & $3.3 \mathrm{E}-05$ & $8.86 \mathrm{E}-05$ \\
\hline $7.30741 \mathrm{E}-05$ & 0.000123746 & $6.52 \mathrm{E}-05$ & 0.000116 & 4.83E-05 & 0.000106 & $2.51 \mathrm{E}-05$ & $9.34 \mathrm{E}-05$ \\
\hline $5.16213 \mathrm{E}-05$ & 0.000133172 & $4.91 \mathrm{E}-05$ & 0.000123 & $3.69 \mathrm{E}-05$ & 0.000111 & $1.85 \mathrm{E}-05$ & $9.65 \mathrm{E}-05$ \\
\hline 0.00002982 & 0.000139138 & $3.19 \mathrm{E}-05$ & 0.000129 & $2.54 \mathrm{E}-05$ & 0.000115 & $1.28 \mathrm{E}-05$ & $9.83 \mathrm{E}-05$ \\
\hline 0.00002982 & 0.000139138 & $3.19 \mathrm{E}-05$ & 0.000129 & $2.54 \mathrm{E}-05$ & 0.000115 & $1.28 \mathrm{E}-05$ & $9.83 \mathrm{E}-05$ \\
\hline
\end{tabular}


Table B3. Radial and tangential WSS values at Slope ratio of 1, Froude Number of 0.1 and Stokes Number of 1 .

\begin{tabular}{|c|c|c|c|c|c|c|c|}
\hline \multicolumn{2}{|c|}{$20 \%$} & \multicolumn{2}{|c|}{$40 \%$} & \multicolumn{2}{|c|}{$60 \%$} & \multicolumn{2}{|c|}{$80 \%$} \\
\hline Radial & Tangential & Radial & Tangential & Radial & Tangential & Radial & Tangential \\
\hline $\mathrm{Pa}$ & $\mathrm{Pa}$ & $\mathrm{Pa}$ & $\mathrm{Pa}$ & $\mathrm{Pa}$ & $\mathrm{Pa}$ & $\mathrm{Pa}$ & $\mathbf{P a}$ \\
\hline $3.73535 \mathrm{E}-05$ & $-6.89808 \mathrm{E}-05$ & -0.0001 & $-9.3 \mathrm{E}-05$ & -0.00015 & $-1 \mathrm{E}-04$ & -0.00013 & -0.0001 \\
\hline$-1.6724 \mathrm{E}-05$ & $-8.04557 \mathrm{E}-05$ & $-5.6 \mathrm{E}-05$ & -0.00013 & $-9.4 \mathrm{E}-05$ & -0.00016 & $-8.5 \mathrm{E}-05$ & -0.00017 \\
\hline $5.4419 \mathrm{E}-06$ & $-8.49492 \mathrm{E}-05$ & $9.96 \mathrm{E}-07$ & -0.00014 & $-1.8 \mathrm{E}-05$ & -0.00019 & $-2.5 \mathrm{E}-05$ & -0.00021 \\
\hline $2.73903 \mathrm{E}-05$ & $-7.80727 \mathrm{E}-05$ & $5.71 \mathrm{E}-05$ & -0.00013 & $6.28 \mathrm{E}-05$ & -0.00018 & $4.38 \mathrm{E}-05$ & -0.00021 \\
\hline $4.21682 \mathrm{E}-05$ & $-5.95909 \mathrm{E}-05$ & 0.000102 & $-9.6 \mathrm{E}-05$ & 0.000138 & -0.00013 & 0.000117 & -0.00015 \\
\hline 0.000048839 & $-4.07418 \mathrm{E}-05$ & 0.000129 & $-5 \mathrm{E}-05$ & 0.000186 & $-5.3 \mathrm{E}-05$ & 0.000172 & $-4.9 \mathrm{E}-05$ \\
\hline $4.63051 \mathrm{E}-05$ & $-2.67277 \mathrm{E}-05$ & 0.000122 & $-6.4 \mathrm{E}-06$ & 0.000193 & $3.06 \mathrm{E}-05$ & 0.00019 & $7.36 \mathrm{E}-05$ \\
\hline $5.42176 \mathrm{E}-05$ & $-9.8891 \mathrm{E}-06$ & $8.49 \mathrm{E}-05$ & $2.09 \mathrm{E}-05$ & 0.000146 & $8.18 \mathrm{E}-05$ & 0.000154 & 0.00016 \\
\hline $4.38084 \mathrm{E}-05$ & $-2.38899 \mathrm{E}-05$ & $5.67 \mathrm{E}-05$ & $2.01 \mathrm{E}-05$ & $6.27 \mathrm{E}-05$ & $5.85 \mathrm{E}-05$ & $7.72 \mathrm{E}-05$ & 0.000122 \\
\hline $3.32092 \mathrm{E}-05$ & $-5.1883 \mathrm{E}-06$ & $5.38 \mathrm{E}-05$ & $1.83 \mathrm{E}-05$ & $2.8 \mathrm{E}-05$ & $2.19 \mathrm{E}-05$ & $1.44 \mathrm{E}-05$ & $2.84 \mathrm{E}-05$ \\
\hline $3.52998 \mathrm{E}-05$ & $-4.4876 \mathrm{E}-06$ & $3.91 \mathrm{E}-05$ & $2.92 \mathrm{E}-05$ & $2.6 \mathrm{E}-05$ & $1.18 \mathrm{E}-05$ & $1.48 \mathrm{E}-05$ & $1.54 \mathrm{E}-05$ \\
\hline $3.82151 \mathrm{E}-05$ & $-1.1341 \mathrm{E}-06$ & $2.86 \mathrm{E}-05$ & $2.29 \mathrm{E}-05$ & $2.46 \mathrm{E}-05$ & $1.03 \mathrm{E}-05$ & $1.68 \mathrm{E}-05$ & $5.63 \mathrm{E}-06$ \\
\hline $3.85472 \mathrm{E}-05$ & $4.8796 \mathrm{E}-06$ & $3.54 \mathrm{E}-05$ & $5.26 \mathrm{E}-06$ & $2.84 \mathrm{E}-05$ & $1.26 \mathrm{E}-05$ & $1.73 \mathrm{E}-05$ & $5.63 \mathrm{E}-06$ \\
\hline $3.55474 \mathrm{E}-05$ & $1.08009 \mathrm{E}-05$ & $3.87 \mathrm{E}-05$ & $3.03 \mathrm{E}-06$ & $2.81 \mathrm{E}-05$ & $1.97 \mathrm{E}-05$ & $1.7 \mathrm{E}-05$ & $7.14 \mathrm{E}-06$ \\
\hline $3.39886 \mathrm{E}-05$ & $1.71775 \mathrm{E}-05$ & $3.73 \mathrm{E}-05$ & $9.82 \mathrm{E}-06$ & $2.73 \mathrm{E}-05$ & $2.06 \mathrm{E}-05$ & $1.62 \mathrm{E}-05$ & $1.14 \mathrm{E}-05$ \\
\hline $3.35044 \mathrm{E}-05$ & 0.000023127 & $3.62 \mathrm{E}-05$ & $1.87 \mathrm{E}-05$ & $2.93 \mathrm{E}-05$ & $1.95 \mathrm{E}-05$ & $1.4 \mathrm{E}-05$ & $1.68 \mathrm{E}-05$ \\
\hline $3.17353 \mathrm{E}-05$ & $2.88378 \mathrm{E}-05$ & $3.51 \mathrm{E}-05$ & $2.52 \mathrm{E}-05$ & $2.7 \mathrm{E}-05$ & $2.17 \mathrm{E}-05$ & $1.08 \mathrm{E}-05$ & $2.24 \mathrm{E}-05$ \\
\hline $2.72979 \mathrm{E}-05$ & $3.28265 \mathrm{E}-05$ & $3.1 \mathrm{E}-05$ & $3.02 \mathrm{E}-05$ & $2.28 \mathrm{E}-05$ & $2.47 \mathrm{E}-05$ & $6.41 \mathrm{E}-06$ & $2.68 \mathrm{E}-05$ \\
\hline $2.00446 \mathrm{E}-05$ & $3.71586 \mathrm{E}-05$ & $2.57 \mathrm{E}-05$ & $3.55 \mathrm{E}-05$ & $1.89 \mathrm{E}-05$ & $2.96 \mathrm{E}-05$ & $5.51 \mathrm{E}-06$ & $2.93 \mathrm{E}-05$ \\
\hline $1.36663 \mathrm{E}-05$ & $4.09091 \mathrm{E}-05$ & $2.01 \mathrm{E}-05$ & $3.99 \mathrm{E}-05$ & $1.52 \mathrm{E}-05$ & $3.39 \mathrm{E}-05$ & $6.04 \mathrm{E}-06$ & $3.01 \mathrm{E}-05$ \\
\hline $6.2684 \mathrm{E}-06$ & $4.29071 \mathrm{E}-05$ & $1.36 \mathrm{E}-05$ & $4.35 \mathrm{E}-05$ & $1.19 \mathrm{E}-05$ & $3.85 \mathrm{E}-05$ & $5.65 \mathrm{E}-06$ & $3.26 \mathrm{E}-05$ \\
\hline$-2.367 \mathrm{E}-06$ & $4.26549 \mathrm{E}-05$ & $5.72 \mathrm{E}-06$ & $4.55 \mathrm{E}-05$ & $7.92 \mathrm{E}-06$ & $4.16 \mathrm{E}-05$ & $4.8 \mathrm{E}-06$ & $3.53 \mathrm{E}-05$ \\
\hline$-1.0687 \mathrm{E}-05$ & $4.07305 \mathrm{E}-05$ & $-3 \mathrm{E}-06$ & $4.45 \mathrm{E}-05$ & $1.43 \mathrm{E}-06$ & $4.38 \mathrm{E}-05$ & $2.49 \mathrm{E}-06$ & $3.94 \mathrm{E}-05$ \\
\hline$-1.8433 \mathrm{E}-05$ & $3.63318 \mathrm{E}-05$ & $-1.2 \mathrm{E}-05$ & $4.14 \mathrm{E}-05$ & $-5.7 \mathrm{E}-06$ & $4.35 \mathrm{E}-05$ & $-1.7 \mathrm{E}-06$ & $4.33 \mathrm{E}-05$ \\
\hline$-2.6201 \mathrm{E}-05$ & $2.98476 \mathrm{E}-05$ & $-1.9 \mathrm{E}-05$ & $3.51 \mathrm{E}-05$ & $-1.3 \mathrm{E}-05$ & $3.75 \mathrm{E}-05$ & $-7.5 \mathrm{E}-06$ & $3.9 \mathrm{E}-05$ \\
\hline$-3.1278 \mathrm{E}-05$ & $2.26188 \mathrm{E}-05$ & $-2.5 \mathrm{E}-05$ & $2.52 \mathrm{E}-05$ & $-1.7 \mathrm{E}-05$ & $2.66 \mathrm{E}-05$ & $-9.8 \mathrm{E}-06$ & $3.07 \mathrm{E}-05$ \\
\hline$-3.4986 \mathrm{E}-05$ & $1.25975 \mathrm{E}-05$ & $-2.8 \mathrm{E}-05$ & $1.7 \mathrm{E}-05$ & $-2.2 \mathrm{E}-05$ & $1.53 \mathrm{E}-05$ & $-9.7 \mathrm{E}-06$ & $2.53 \mathrm{E}-05$ \\
\hline$-3.5332 \mathrm{E}-05$ & $2.8234 \mathrm{E}-06$ & $-4.5 \mathrm{E}-05$ & $1.05 \mathrm{E}-05$ & $-6.1 \mathrm{E}-05$ & $5.08 \mathrm{E}-05$ & $-3.2 \mathrm{E}-05$ & 0.000114 \\
\hline$-3.6982 \mathrm{E}-05$ & -0.000005259 & $-8.7 \mathrm{E}-05$ & $1.86 \mathrm{E}-05$ & -0.00011 & $8.49 \mathrm{E}-05$ & $-8.5 \mathrm{E}-05$ & 0.000146 \\
\hline$-4.1844 \mathrm{E}-05$ & $-1.98888 \mathrm{E}-05$ & -0.00011 & $5.37 \mathrm{E}-06$ & -0.00015 & $7.32 \mathrm{E}-05$ & -0.00013 & 0.000108 \\
\hline$-5.0113 \mathrm{E}-0 \mathrm{~S}$ & $-3.52024 \mathrm{E}-05$ & -0.00013 & $-2.4 \mathrm{E}-05$ & -0.00018 & $2.29 \mathrm{E}-05$ & -0.00015 & $3.8 \mathrm{E}-05$ \\
\hline$-4.9425 \mathrm{E}-05$ & $-5.56658 \mathrm{E}-05$ & -0.00012 & $-6.5 E-05$ & -0.00017 & $-4.6 \mathrm{E}-05$ & -0.00015 & $-4 \mathrm{E}-05$ \\
\hline $3.64917 \mathrm{E}-05$ & $-7.05884 \mathrm{E}-05$ & $-9.3 \mathrm{E}-05$ & -0.0001 & -0.00014 & -0.00011 & -0.00012 & -0.00012 \\
\hline
\end{tabular}


Table B4. Radial and tangential WSS values at Slope ratio of 0.1, Froude Number of 1.2 and Stokes Number of 1 .

\begin{tabular}{|c|c|c|c|c|c|c|c|}
\hline \multicolumn{2}{|c|}{$20 \%$} & \multicolumn{2}{|c|}{$40 \%$} & \multicolumn{2}{|c|}{$60 \%$} & \multicolumn{2}{|c|}{$80 \%$} \\
\hline Radial & Tangential & Radial & Tangential & Radial & Tangential & Radial & Tangential \\
\hline $\mathrm{Pa}$ & $\mathrm{Pa}$ & $\mathrm{Pa}$ & $\mathrm{Pa}$ & $\mathrm{Pa}$ & $\mathrm{Pa}$ & $\mathrm{Pa}$ & $\mathrm{Pa}$ \\
\hline-0.02524 & 0.027378 & -0.02049 & 0.023714 & -0.0154 & 0.021769 & -0.00668 & 0.016536 \\
\hline-0.02971 & 0.022149 & -0.02435 & 0.01875 & -0.01766 & 0.017413 & -0.00702 & 0.012292 \\
\hline-0.03306 & 0.016054 & -0.02735 & 0.013069 & -0.01929 & 0.012392 & -0.00709 & 0.00756 \\
\hline-0.03523 & 0.00929 & -0.02945 & 0.006821 & -0.02034 & 0.006818 & -0.007 & 0.002441 \\
\hline-0.03605 & 0.002121 & -0.03047 & 0.000227 & -0.02063 & 0.000868 & -0.00661 & -0.0029 \\
\hline-0.0355 & -0.0052 & -0.03036 & -0.00652 & -0.02017 & -0.00527 & -0.00599 & -0.00832 \\
\hline-0.03358 & -0.01235 & -0.0291 & -0.0131 & -0.01891 & -0.01139 & -0.0051 & -0.01358 \\
\hline-0.03038 & -0.01909 & -0.02669 & -0.01931 & -0.01688 & -0.01725 & -0.00397 & -0.01852 \\
\hline-0.02592 & -0.02516 & -0.02316 & -0.0249 & -0.01408 & -0.0226 & -0.00264 & -0.02289 \\
\hline-0.02038 & -0.03025 & -0.0186 & -0.02957 & -0.01061 & -0.02714 & -0.00111 & -0.02646 \\
\hline-0.01395 & -0.0342 & -0.01315 & -0.03313 & -0.00655 & -0.03069 & 0.000549 & -0.02904 \\
\hline-0.00686 & -0.03677 & -0.00703 & -0.03533 & -0.00207 & -0.03297 & 0.002279 & -0.03039 \\
\hline 0.000573 & -0.03781 & -0.00048 & -0.03599 & 0.002635 & -0.03379 & 0.003962 & -0.03037 \\
\hline 0.007987 & -0.03728 & 0.00613 & -0.03507 & 0.007283 & -0.03305 & 0.005491 & -0.02894 \\
\hline 0.015145 & -0.03512 & 0.012555 & -0.03249 & 0.01168 & -0.03069 & 0.006843 & -0.02603 \\
\hline 0.021625 & -0.03148 & 0.018375 & -0.02844 & 0.015523 & -0.02687 & 0.00785 & -0.02182 \\
\hline 0.027127 & -0.02649 & 0.023306 & -0.02307 & 0.018583 & -0.02176 & 0.008456 & -0.01654 \\
\hline 0.031512 & -0.02045 & 0.027186 & -0.01676 & 0.020769 & -0.01575 & 0.008651 & -0.01061 \\
\hline 0.034527 & -0.0136 & 0.029801 & -0.00981 & 0.021951 & -0.00916 & 0.008406 & -0.00435 \\
\hline 0.036108 & -0.00626 & 0.031124 & -0.00255 & 0.022174 & -0.00234 & 0.007814 & 0.001913 \\
\hline 0.036196 & 0.001271 & 0.031118 & 0.004687 & 0.021471 & 0.004386 & 0.006938 & 0.007899 \\
\hline 0.034816 & 0.008674 & 0.029837 & 0.011579 & 0.019901 & 0.010741 & 0.005816 & 0.013336 \\
\hline 0.032046 & 0.015675 & 0.027368 & 0.017853 & 0.017576 & 0.016457 & 0.004459 & 0.018054 \\
\hline 0.028044 & 0.022011 & 0.023905 & 0.023323 & 0.014679 & 0.021388 & 0.003064 & 0.021907 \\
\hline 0.022983 & 0.027367 & 0.019596 & 0.027725 & 0.011322 & 0.025305 & 0.001588 & 0.024753 \\
\hline 0.017093 & 0.031671 & 0.014643 & 0.031063 & 0.007647 & 0.028246 & $9.17 \mathrm{E}-05$ & 0.026662 \\
\hline 0.010623 & 0.034716 & 0.009258 & 0.033188 & 0.003817 & 0.030091 & -0.00134 & 0.027549 \\
\hline 0.003794 & 0.036439 & 0.003612 & 0.034095 & $-6.6 \mathrm{E}-05$ & 0.030861 & -0.00265 & 0.027465 \\
\hline-0.00312 & 0.036812 & -0.00209 & 0.033815 & -0.00387 & 0.0306 & -0.00384 & 0.026496 \\
\hline-0.00984 & 0.035815 & -0.00765 & 0.032367 & -0.00746 & 0.029312 & -0.00484 & 0.024674 \\
\hline-0.01629 & 0.033538 & -0.01296 & 0.029833 & -0.01082 & 0.0271 & -0.0057 & 0.022088 \\
\hline-0.02209 & 0.030009 & -0.01782 & 0.026269 & -0.0138 & 0.023997 & -0.00636 & 0.018793 \\
\hline-0.0267 & 0.02584 & -0.02174 & 0.022241 & -0.01615 & 0.020479 & -0.00681 & 0.015267 \\
\hline
\end{tabular}


Table B5. Radial and tangential WSS values at Slope ratio of 1, Froude Number of 1.2 and Stokes Number of 1 .

\begin{tabular}{|c|c|c|c|c|c|c|c|}
\hline \multicolumn{2}{|c|}{$20 \%$} & \multicolumn{2}{|c|}{$40 \%$} & \multicolumn{2}{|c|}{$60 \%$} & \multicolumn{2}{|c|}{$80 \%$} \\
\hline Radial & Tangential & Radial & Tangential & Radial & Tangential & Radial & Tangential \\
\hline $\mathrm{Pa}$ & $\mathrm{Pa}$ & $\mathrm{Pa}$ & $\mathrm{Pa}$ & $\mathrm{Pa}$ & $\mathrm{Pa}$ & $\mathrm{Pa}$ & $\mathrm{Pa}$ \\
\hline-0.00226 & 0.001408 & -0.00243 & 0.001683 & -0.00193 & 0.001776 & -0.00071 & 0.00117 \\
\hline-0.00243 & 0.001071 & -0.00256 & 0.001383 & -0.00198 & 0.001478 & -0.00069 & 0.000863 \\
\hline-0.00256 & 0.000694 & -0.00265 & 0.001043 & -0.00199 & 0.001091 & -0.00065 & 0.000523 \\
\hline-0.00265 & 0.000304 & -0.00269 & 0.00068 & -0.00202 & 0.000709 & -0.00059 & 0.000168 \\
\hline-0.00266 & -0.00011 & -0.00264 & 0.00025 & -0.00194 & 0.000323 & -0.00052 & -0.00021 \\
\hline-0.00258 & -0.00052 & -0.00259 & -0.00019 & -0.00177 & $-8.2 \mathrm{E}-05$ & -0.00044 & -0.0006 \\
\hline-0.0024 & -0.00093 & -0.00242 & -0.00069 & -0.00158 & -0.00054 & -0.00038 & -0.00099 \\
\hline-0.00207 & -0.00134 & -0.00217 & -0.00117 & -0.00133 & -0.00106 & -0.00031 & -0.00141 \\
\hline-0.0016 & -0.00179 & -0.00177 & -0.0016 & -0.00105 & -0.0016 & -0.00025 & -0.0018 \\
\hline-0.00124 & -0.00202 & -0.00133 & -0.00196 & -0.00074 & -0.0021 & -0.00017 & -0.00211 \\
\hline-0.00081 & -0.00216 & -0.00086 & -0.00226 & -0.00043 & -0.0025 & $-5.9 \mathrm{E}-05$ & -0.00241 \\
\hline-0.00031 & -0.00225 & -0.00032 & -0.00244 & $3.94 \mathrm{E}-07$ & -0.00267 & 0.000131 & -0.00264 \\
\hline 0.00023 & -0.00218 & 0.000295 & -0.00238 & 0.000532 & -0.00269 & 0.000419 & -0.00263 \\
\hline 0.000723 & -0.00193 & 0.000835 & -0.00205 & 0.001022 & -0.00226 & 0.000725 & -0.00217 \\
\hline 0.001095 & -0.00154 & 0.001186 & -0.00153 & 0.001284 & -0.00157 & 0.000836 & -0.00137 \\
\hline 0.00141 & -0.00109 & 0.001436 & -0.00095 & 0.001414 & -0.00087 & 0.000807 & -0.00056 \\
\hline 0.001629 & -0.00061 & 0.001558 & -0.00035 & 0.001401 & -0.00021 & 0.000692 & 0.000138 \\
\hline 0.0017 & -0.00013 & 0.001495 & 0.000214 & 0.001214 & 0.000342 & 0.000497 & 0.000638 \\
\hline 0.001692 & 0.000373 & 0.001355 & 0.000767 & 0.00098 & 0.000839 & 0.000314 & 0.001032 \\
\hline 0.001565 & 0.000867 & 0.00113 & 0.001265 & 0.000723 & 0.00125 & 0.000154 & 0.001339 \\
\hline 0.001339 & 0.00128 & 0.000838 & 0.001657 & 0.00046 & 0.001557 & $2.25 \mathrm{E}-05$ & 0.001543 \\
\hline 0.001059 & 0.001574 & 0.000526 & 0.001913 & 0.00021 & 0.001756 & $-8.3 \mathrm{E}-05$ & 0.001659 \\
\hline 0.000764 & 0.00181 & 0.000229 & 0.002107 & $-1.2 \mathrm{E}-05$ & 0.001919 & -0.00016 & 0.001751 \\
\hline 0.000454 & 0.002044 & $-6.8 \mathrm{E}-05$ & 0.002297 & -0.00023 & 0.002092 & -0.00024 & 0.001857 \\
\hline 0.000112 & 0.002204 & -0.00038 & 0.002412 & -0.00046 & 0.002203 & -0.00031 & 0.00191 \\
\hline-0.00023 & 0.002252 & -0.00068 & 0.002422 & -0.00068 & 0.002237 & -0.00039 & 0.0019 \\
\hline-0.00057 & 0.002249 & -0.00097 & 0.002401 & -0.00089 & 0.00225 & -0.00045 & 0.001877 \\
\hline-0.00092 & 0.002201 & -0.00127 & 0.002345 & -0.0011 & 0.002232 & -0.00052 & 0.001823 \\
\hline-0.00128 & 0.002102 & -0.00156 & 0.002244 & -0.00131 & 0.002164 & -0.00058 & 0.001724 \\
\hline-0.00161 & 0.001945 & -0.00181 & 0.002098 & -0.00149 & 0.002043 & -0.00063 & 0.001584 \\
\hline-0.00187 & 0.001736 & -0.00202 & 0.001913 & -0.00165 & 0.00189 & -0.00065 & 0.001414 \\
\hline-0.0021 & 0.001454 & -0.00221 & 0.001665 & -0.00177 & 0.001666 & -0.00067 & 0.001196 \\
\hline-0.00222 & 0.0012 & -0.00231 & 0.001432 & -0.00184 & 0.001452 & -0.00066 & 0.00099 \\
\hline
\end{tabular}


Table B6. Radial and tangential WSS values at Slope ratio of 2.4, Froude Number of 1.2 and Stokes Number of 1.

\begin{tabular}{|c|c|c|c|c|c|c|c|}
\hline \multicolumn{2}{|c|}{$20 \%$} & \multicolumn{2}{|c|}{$40 \%$} & \multicolumn{2}{|c|}{$60 \%$} & \multicolumn{2}{|c|}{$80 \%$} \\
\hline Radial & Tangential & Radial & Tangential & Radial & Tangential & Radial & Tangential \\
\hline $\mathrm{Pa}$ & $\mathrm{Pa}$ & $\mathrm{Pa}$ & $\mathrm{Pa}$ & $\mathrm{Pa}$ & $\mathrm{Pa}$ & $\mathrm{Pa}$ & $\mathrm{Pa}$ \\
\hline-0.00021 & 0.002371 & -0.0011 & 0.002827 & -0.00208 & 0.003056 & -0.00169 & 0.002263 \\
\hline-0.001 & 0.002026 & -0.00166 & 0.002434 & -0.00229 & 0.00255 & -0.00157 & 0.001703 \\
\hline-0.00128 & 0.00231 & -0.00184 & 0.002724 & -0.00212 & 0.00269 & -0.00128 & 0.001658 \\
\hline-0.00164 & 0.001998 & -0.00209 & 0.002455 & -0.00201 & 0.002337 & -0.00103 & 0.001184 \\
\hline-0.00217 & 0.001839 & -0.00245 & 0.002346 & -0.00203 & 0.002112 & -0.00087 & 0.000995 \\
\hline-0.00256 & 0.001068 & -0.0028 & 0.001682 & -0.00218 & 0.001497 & -0.00081 & 0.000627 \\
\hline-0.00305 & 0.000989 & -0.00343 & 0.001512 & -0.00292 & 0.001535 & -0.00123 & 0.001034 \\
\hline-0.00273 & 0.000274 & -0.0033 & 0.000532 & -0.00354 & 0.000832 & -0.00191 & 0.000998 \\
\hline-0.00299 & $2.48 \mathrm{E}-05$ & -0.00355 & $7.07 \mathrm{E}-05$ & -0.00402 & $8.82 \mathrm{E}-05$ & -0.00353 & 0.000793 \\
\hline-0.00256 & -0.00081 & -0.00348 & -0.00084 & -0.00407 & -0.00123 & -0.0045 & -0.00044 \\
\hline-0.00245 & -0.00111 & -0.00382 & -0.00159 & -0.0051 & -0.00176 & -0.00345 & -0.00158 \\
\hline .0 .00162 & -0.00102 & -0.00263 & -0.00102 & -0.0039 & -0.0008 & -0.00389 & -0.0018 \\
\hline-0.00136 & -0.00148 & -0.00237 & -0.00122 & -0.00284 & -0.00046 & -0.00061 & 0.000517 \\
\hline-0.0008 & -0.00149 & -0.00184 & -0.00118 & -0.00219 & -0.00059 & -0.00012 & 0.001123 \\
\hline-0.00063 & -0.00107 & -0.0016 & -0.00028 & -0.00213 & 0.000914 & -0.001 & 0.002573 \\
\hline-0.00048 & -0.00098 & -0.00156 & $5.2 \mathrm{E}-05$ & -0.00243 & 0.001324 & -0.00142 & 0.002674 \\
\hline $6.86 \mathrm{E}-05$ & -0.00069 & -0.00125 & 0.000476 & -0.00233 & 0.001918 & -0.00122 & 0.003002 \\
\hline 0.000115 & -0.00054 & -0.00144 & 0.000648 & -0.00272 & 0.002098 & -0.00138 & 0.002929 \\
\hline 0.000241 & -0.00038 & -0.00141 & 0.000702 & -0.0027 & 0.001913 & -0.00147 & 0.002366 \\
\hline 0.00053 & -0.00022 & -0.00118 & 0.000858 & -0.00248 & 0.00203 & -0.00145 & 0.002395 \\
\hline 0.000654 & 0.000192 & -0.00116 & 0.001257 & -0.00252 & 0.002381 & -0.00151 & 0.002695 \\
\hline 0.000938 & 0.000391 & -0.00102 & 0.00142 & -0.0025 & 0.00253 & -0.00147 & 0.002798 \\
\hline 0.001136 & 0.000759 & -0.00085 & 0.001731 & -0.00246 & 0.002863 & -0.0015 & 0.003096 \\
\hline 0.000954 & 0.001277 & -0.00089 & 0.002197 & -0.00251 & 0.003308 & -0.00165 & 0.003336 \\
\hline 0.001005 & 0.001549 & -0.00074 & 0.002504 & -0.00211 & 0.003513 & -0.00191 & 0.003173 \\
\hline 0.000712 & 0.002108 & -0.00084 & 0.002955 & -0.0023 & 0.003751 & -0.00214 & 0.003502 \\
\hline 0.000389 & 0.002664 & -0.00094 & 0.003279 & -0.00222 & 0.003721 & -0.00198 & 0.003287 \\
\hline$-8.4 \mathrm{E}-05$ & 0.00247 & -0.00156 & 0.002904 & -0.0021 & 0.003273 & -0.00175 & 0.002621 \\
\hline-0.00031 & 0.00271 & -0.00141 & 0.003033 & -0.002 & 0.003167 & -0.00142 & 0.002424 \\
\hline-0.00082 & 0.002804 & -0.00153 & 0.002955 & -0.00204 & 0.002858 & -0.00121 & 0.002025 \\
\hline-0.00141 & 0.002656 & -0.00181 & 0.00277 & -0.00207 & 0.002535 & -0.00108 & 0.001649 \\
\hline-0.00173 & 0.002253 & -0.00207 & 0.002485 & -0.00204 & 0.002231 & -0.00099 & 0.001322 \\
\hline-0.00173 & 0.002253 & -0.00207 & 0.002485 & -0.00204 & 0.002231 & -0.00099 & 0.001322 \\
\hline
\end{tabular}


Table B7. Radial and tangential WSS values at Slope ratio of 3, Froude Number of 1.2 and Stokes Number of 1.

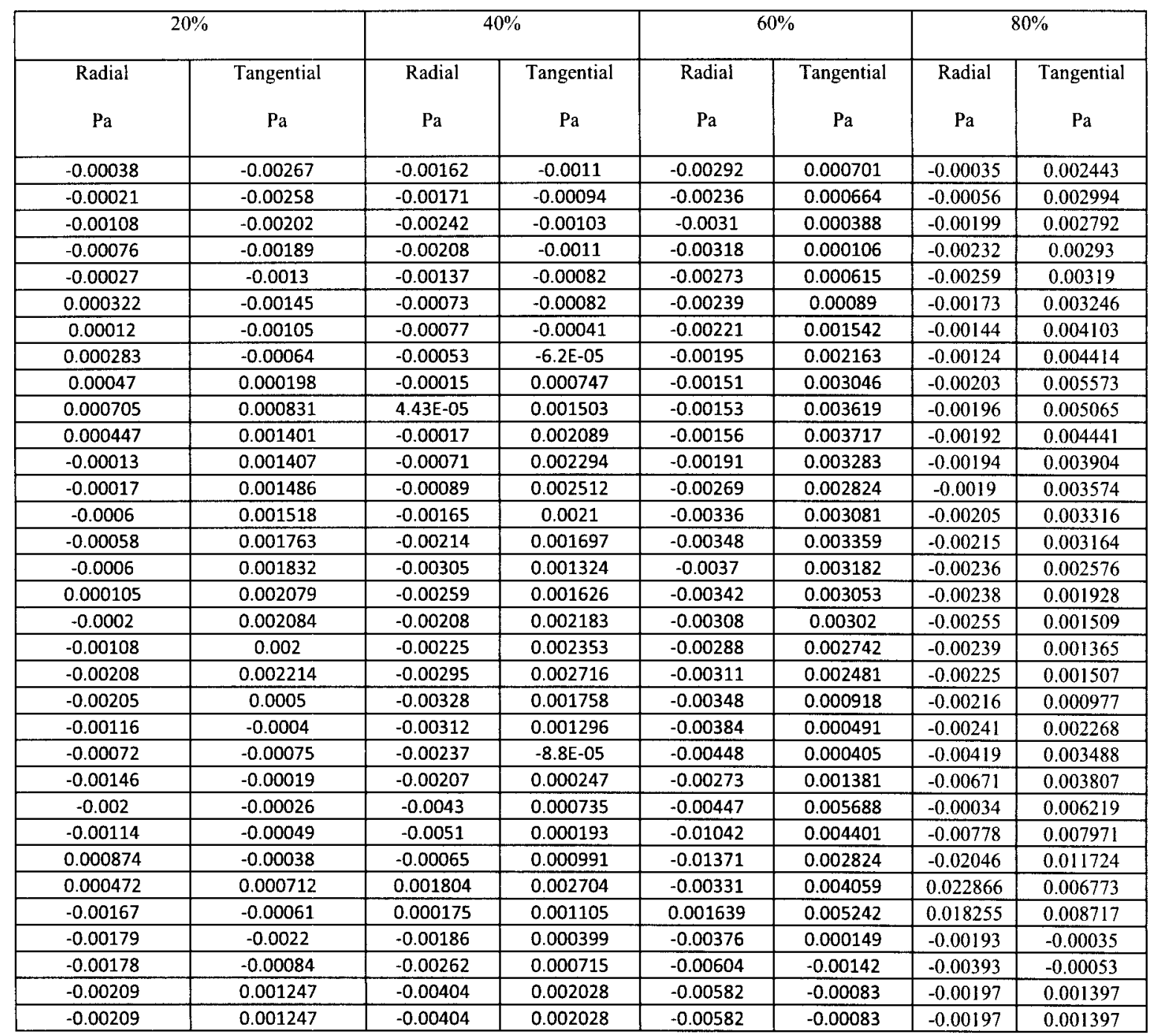




\section{B.2. WSS data as a function of Froude Number at different combinations of}

\section{constant Stokes Number and constant Slope ratio}

Table B8. Radial and tangential WSS values at Froude Number of 0.1, Stokes Number of

1 and Slope ratio of 0.1 .

\begin{tabular}{|c|c|c|c|c|c|c|c|}
\hline \multicolumn{2}{|c|}{$20 \%$} & \multicolumn{2}{|c|}{$40 \%$} & \multicolumn{2}{|c|}{$60 \%$} & \multicolumn{2}{|c|}{$80 \%$} \\
\hline Radial & Tangential & Radial & Tangential & Radial & Tangential & Radial & Tangential \\
\hline $\mathrm{Pa}$ & $\mathrm{Pa}$ & $\mathrm{Pa}$ & $\mathrm{Pa}$ & $\mathrm{Pa}$ & $\mathrm{Pa}$ & $\mathrm{Pa}$ & $\mathrm{Pa}$ \\
\hline$-3.4095 \mathrm{E}-05$ & $-1.96741 \mathrm{E}-05$ & $-2.8 \mathrm{E}-05$ & $-1.6 \mathrm{E}-05$ & $-1.8 \mathrm{E}-05$ & $-1.2 \mathrm{E}-05$ & $-9.8 \mathrm{E}-06$ & $-1 \mathrm{E}-05$ \\
\hline$-3.055 \mathrm{E}-05$ & $-2.24822 \mathrm{E}-05$ & $-2.5 \mathrm{E}-05$ & $-2.2 \mathrm{E}-05$ & $-1.8 \mathrm{E}-05$ & $-1.7 \mathrm{E}-05$ & $-9.3 \mathrm{E}-06$ & $-1.5 \mathrm{E}-05$ \\
\hline$-2.8509 \mathrm{E}-05$ & -0.000018969 & $-2.3 \mathrm{E}-05$ & $-2.1 \mathrm{E}-05$ & $-1.6 \mathrm{E}-05$ & $-1.7 \mathrm{E}-05$ & $-8.2 \mathrm{E}-06$ & $-1.5 \mathrm{E}-05$ \\
\hline$-2.3288 \mathrm{E}-05$ & $-2.34786 \mathrm{E}-05$ & $-1.7 \mathrm{E}-05$ & $-2.6 \mathrm{E}-05$ & $-1.3 \mathrm{E}-05$ & $-2.3 \mathrm{E}-05$ & $-5.7 \mathrm{E}-06$ & $-2 \mathrm{E}-05$ \\
\hline$-1.1674 \mathrm{E}-05$ & $-3.51235 \mathrm{E}-05$ & $-5.6 \mathrm{E}-06$ & $-3.8 \mathrm{E}-05$ & $-4.1 \mathrm{E}-06$ & $-3.5 \mathrm{E}-05$ & $-1.3 \mathrm{E}-06$ & $-3.1 \mathrm{E}-05$ \\
\hline$-1.0366 \mathrm{E}-06$ & $-3.91362 \mathrm{E}-05$ & $5.4 \mathrm{E}-06$ & $-4.1 \mathrm{E}-05$ & $4.06 \mathrm{E}-06$ & $-3.9 \mathrm{E}-05$ & $2.98 \mathrm{E}-06$ & $-3.5 \mathrm{E}-05$ \\
\hline $1.6595 \mathrm{E}-06$ & $-3.24136 \mathrm{E}-05$ & $9.63 \mathrm{E}-06$ & $-3.3 \mathrm{E}-05$ & $8.03 \mathrm{E}-06$ & $-3.2 \mathrm{E}-05$ & $5.36 \mathrm{E}-06$ & $-2.9 \mathrm{E}-05$ \\
\hline $4.8589 \mathrm{E}-06$ & $-2.58131 \mathrm{E}-05$ & $1.21 \mathrm{E}-05$ & $-2.5 \mathrm{E}-05$ & $8.78 \mathrm{E}-06$ & $-2.4 \mathrm{E}-05$ & $5.27 \mathrm{E}-06$ & $-2.1 \mathrm{E}-05$ \\
\hline 0.000013642 & $-2.49991 \mathrm{E}-05$ & $2.14 \mathrm{E}-05$ & $-2.5 \mathrm{E}-05$ & $1.72 \mathrm{E}-05$ & $-2.4 \mathrm{E}-05$ & $1.08 \mathrm{E}-05$ & $-2.2 \mathrm{E}-05$ \\
\hline $2.42501 \mathrm{E}-05$ & -0.000026129 & $3.2 \mathrm{E}-05$ & $-2.3 \mathrm{E}-05$ & $2.67 \mathrm{E}-05$ & $-2.2 \mathrm{E}-05$ & $1.66 \mathrm{E}-05$ & $-2 \mathrm{E}-05$ \\
\hline $2.72711 \mathrm{E}-05$ & $-2.36395 \mathrm{E}-05$ & 3.3E-05 & $-1.8 \mathrm{E}-05$ & 2.79E-05 & $-1.5 \mathrm{E}-05$ & $1.77 \mathrm{E}-05$ & $-1.3 \mathrm{E}-05$ \\
\hline $2.92746 \mathrm{E}-05$ & $-1.39336 \mathrm{E}-05$ & $3.14 \mathrm{E}-05$ & $-8.5 \mathrm{E}-06$ & $2.54 \mathrm{E}-05$ & $-3.8 \mathrm{E}-06$ & $1.74 \mathrm{E}-05$ & $-8.3 \mathrm{E}-07$ \\
\hline $3.42269 \mathrm{E}-05$ & $-1.6084 \mathrm{E}-06$ & $3.29 \mathrm{E}-05$ & $2.09 \mathrm{E}-06$ & $2.42 \mathrm{E}-05$ & $4.58 \mathrm{E}-06$ & $1.62 \mathrm{E}-05$ & $8.06 \mathrm{E}-06$ \\
\hline $3.72065 \mathrm{E}-05$ & $-1.2868 \mathrm{E}-06$ & $3.51 \mathrm{E}-05$ & $2.36 \mathrm{E}-06$ & $2.4 \mathrm{E}-05$ & $2.85 \mathrm{E}-06$ & $1.57 \mathrm{E}-05$ & $5.52 \mathrm{E}-06$ \\
\hline $3.86595 \mathrm{E}-05$ & $-5.1118 \mathrm{E}-06$ & $3.66 \mathrm{E}-05$ & $-2.2 \mathrm{E}-06$ & $2.52 \mathrm{E}-05$ & $-4.3 \mathrm{E}-06$ & $1.71 \mathrm{E}-05$ & $-3.5 \mathrm{E}-06$ \\
\hline $3.48984 \mathrm{E}-05$ & $8.1534 \mathrm{E}-06$ & $3.59 \mathrm{E}-05$ & $4.36 \mathrm{E}-06$ & $2.8 \mathrm{E}-05$ & $-4.2 \mathrm{E}-09$ & $2.24 \mathrm{E}-05$ & $-4.4 \mathrm{E}-06$ \\
\hline $2.91362 \mathrm{E}-05$ & $2.54333 \mathrm{E}-05$ & $3.59 \mathrm{E}-05$ & $1.36 \mathrm{E}-05$ & $2.99 \mathrm{E}-05$ & $6.67 \mathrm{E}-06$ & $2.69 \mathrm{E}-05$ & $-2.2 \mathrm{E}-08$ \\
\hline $2.71421 \mathrm{E}-05$ & $2.91528 \mathrm{E}-05$ & $3.76 \mathrm{E}-05$ & $1.45 \mathrm{E}-05$ & $3.39 \mathrm{E}-05$ & $1.02 \mathrm{E}-05$ & $3.24 \mathrm{E}-05$ & $-1.5 \mathrm{E}-06$ \\
\hline 0.00002718 & $2.65671 \mathrm{E}-05$ & $3.96 \mathrm{E}-05$ & $1.35 \mathrm{E}-05$ & $3.73 \mathrm{E}-05$ & $1.02 \mathrm{E}-05$ & $3.57 \mathrm{E}-05$ & $-2.9 \mathrm{E}-06$ \\
\hline $2.31082 \mathrm{E}-05$ & $2.86146 \mathrm{E}-05$ & $3.43 \mathrm{E}-05$ & $1.9 \mathrm{E}-05$ & $3.72 \mathrm{E}-05$ & $1.43 \mathrm{E}-05$ & $3.71 \mathrm{E}-05$ & $-2.1 \mathrm{E}-07$ \\
\hline $1.84308 \mathrm{E}-05$ & $3.28332 \mathrm{E}-05$ & $2.71 \mathrm{E}-05$ & $2.93 \mathrm{E}-05$ & $3.39 \mathrm{E}-05$ & $2.44 \mathrm{E}-05$ & $3.52 \mathrm{E}-05$ & $7.57 \mathrm{E}-06$ \\
\hline $1.82657 \mathrm{E}-05$ & 3.65255E-05 & $2.38 \mathrm{E}-05$ & $3.73 \mathrm{E}-05$ & $3.08 \mathrm{E}-05$ & $3.27 \mathrm{E}-05$ & $3.2 \mathrm{E}-05$ & $1.54 \mathrm{E}-05$ \\
\hline $2.14862 \mathrm{E}-05$ & $3.86382 \mathrm{E}-05$ & $2.12 \mathrm{E}-05$ & $3.96 \mathrm{E}-05$ & $2.87 \mathrm{E}-05$ & $3.67 \mathrm{E}-05$ & $3 \mathrm{E}-05$ & $1.99 \mathrm{E}-05$ \\
\hline $1.31388 \mathrm{E}-05$ & $3.47353 \mathrm{E}-05$ & $1.36 \mathrm{E}-05$ & $3.74 \mathrm{E}-05$ & $2.32 \mathrm{E}-05$ & $3.83 \mathrm{E}-05$ & $2.61 \mathrm{E}-05$ & $2.38 \mathrm{E}-05$ \\
\hline$-7.5596 \mathrm{E}-06$ & $3.20852 \mathrm{E}-05$ & $1.23 \mathrm{E}-06$ & $3.98 \mathrm{E}-05$ & $1.37 \mathrm{E}-05$ & $4.15 \mathrm{E}-05$ & $1.71 \mathrm{E}-05$ & $2.99 \mathrm{E}-05$ \\
\hline$-2.624 \mathrm{E}-05$ & $2.69493 \mathrm{E}-05$ & $-4.5 \mathrm{E}-06$ & $4.57 \mathrm{E}-05$ & $1.48 \mathrm{E}-06$ & $4.16 \mathrm{E}-05$ & $6.18 \mathrm{E}-06$ & $3.42 \mathrm{E}-05$ \\
\hline$-3.5234 \mathrm{E}-05$ & 0.000019437 & $-1.7 \mathrm{E}-05$ & $3.87 \mathrm{E}-05$ & $-1.4 \mathrm{E}-05$ & $3.61 \mathrm{E}-05$ & $-1.9 \mathrm{E}-06$ & $3.45 \mathrm{E}-05$ \\
\hline$-3.3242 \mathrm{E}-05$ & 0.000013498 & $-2.4 \mathrm{E}-05$ & $2.64 \mathrm{E}-05$ & $-1.5 \mathrm{E}-05$ & $3.11 \mathrm{E}-05$ & $-5 \mathrm{E}-06$ & $3.13 \mathrm{E}-05$ \\
\hline$-2.9175 \mathrm{E}-05$ & $1.55634 \mathrm{E}-05$ & $-2.3 \mathrm{E}-05$ & $2.29 \mathrm{E}-05$ & $-1.6 \mathrm{E}-05$ & $1.94 \mathrm{E}-05$ & $-1.5 \mathrm{E}-05$ & $1.98 \mathrm{E}-05$ \\
\hline$-3.9157 \mathrm{E}-05$ & $8.4424 \mathrm{E}-06$ & $-2.7 \mathrm{E}-05$ & $1.87 \mathrm{E}-05$ & $-1.6 \mathrm{E}-05$ & $1.8 \mathrm{E}-05$ & $-1.5 \mathrm{E}-05$ & $1.4 \mathrm{E}-05$ \\
\hline$-4.2303 \mathrm{E}-05$ & 0.000003878 & $-3 \mathrm{E}-05$ & $9.83 \mathrm{E}-06$ & $-2.4 \mathrm{E}-05$ & $1.66 \mathrm{E}-05$ & $-1.3 \mathrm{E}-05$ & $1.37 \mathrm{E}-05$ \\
\hline$-3.7409 \mathrm{E}-05$ & $-6.5454 \mathrm{E}-06$ & $-3.1 \mathrm{E}-05$ & $-7.3 \mathrm{E}-06$ & $-2.2 \mathrm{E}-05$ & $3.49 \mathrm{E}-06$ & $-1.4 \mathrm{E}-05$ & $2.39 \mathrm{E}-06$ \\
\hline$-3.0497 \mathrm{E}-05$ & $-2.31608 \mathrm{E}-05$ & $-2.7 \mathrm{E}-05$ & $-1.5 \mathrm{E}-05$ & $-1.8 \mathrm{E}-05$ & $-1.3 \mathrm{E}-05$ & $-1.1 \mathrm{E}-05$ & $-1.1 \mathrm{E}-05$ \\
\hline
\end{tabular}


Table B9. Radial and tangential WSS values at Froude Number of 0.64, Stokes Number of 1 and Slope ratio of 0.1 .

\begin{tabular}{|c|c|c|c|c|c|c|c|}
\hline \multicolumn{2}{|c|}{$20 \%$} & \multicolumn{2}{|c|}{$40 \%$} & \multicolumn{2}{|c|}{$60 \%$} & \multicolumn{2}{|c|}{$80 \%$} \\
\hline $\begin{array}{c}\text { Radial } \\
\mathrm{Pa}\end{array}$ & $\begin{array}{c}\text { Tangential } \\
\mathbf{P a}\end{array}$ & $\begin{array}{c}\text { Radial } \\
\mathrm{Pa}\end{array}$ & $\begin{array}{c}\text { Tangential } \\
\mathrm{Pa}\end{array}$ & $\begin{array}{c}\text { Radial } \\
\mathrm{Pa}\end{array}$ & $\begin{array}{c}\text { Tangential } \\
\text { Pa }\end{array}$ & $\begin{array}{c}\text { Radial } \\
\mathrm{Pa}\end{array}$ & $\begin{array}{c}\text { Tangential } \\
\mathrm{Pa}\end{array}$ \\
\hline 0.004724 & 0.001906 & 0.004043 & 0.002173 & 0.002986 & 0.002261 & 0.001677 & 0.002148 \\
\hline 0.004226 & 0.002783 & 0.003538 & 0.002938 & 0.002578 & 0.002908 & 0.001443 & 0.002698 \\
\hline 0.003587 & 0.003534 & 0.002936 & 0.003578 & 0.002107 & 0.003452 & 0.001168 & 0.003167 \\
\hline 0.002837 & 0.004136 & 0.00226 & 0.004078 & 0.001582 & 0.003871 & 0.000862 & 0.003522 \\
\hline 0.002005 & 0.004576 & 0.00153 & 0.004427 & 0.001027 & 0.004154 & 0.000539 & 0.003753 \\
\hline 0.001124 & 0.004847 & 0.000775 & 0.00462 & 0.00046 & 0.004294 & 0.000216 & 0.003857 \\
\hline 0.000223 & 0.004946 & $1.87 \mathrm{E}-05$ & 0.004658 & -0.0001 & 0.004293 & $-9.6 \mathrm{E}-05$ & 0.003835 \\
\hline-0.00067 & 0.004876 & -0.00072 & 0.004547 & -0.00064 & 0.00416 & -0.00039 & 0.003699 \\
\hline-0.00152 & 0.004648 & -0.00141 & 0.004301 & -0.00113 & 0.00391 & -0.00065 & 0.003462 \\
\hline-0.00232 & 0.00427 & -0.00204 & 0.003933 & -0.00158 & 0.003557 & -0.00089 & 0.00314 \\
\hline-0.00303 & 0.003761 & -0.0026 & 0.003462 & -0.00197 & 0.003121 & -0.0011 & 0.002749 \\
\hline-0.00365 & 0.003134 & -0.00308 & 0.002908 & -0.0023 & 0.002625 & -0.00128 & 0.002312 \\
\hline-0.00415 & 0.002408 & -0.00347 & 0.002289 & -0.00256 & 0.002096 & -0.00143 & 0.001856 \\
\hline-0.00453 & 0.001602 & -0.00379 & 0.00162 & -0.00279 & 0.001561 & -0.00155 & 0.001431 \\
\hline-0.00476 & 0.000732 & -0.00403 & 0.000904 & -0.00301 & 0.001026 & -0.00173 & 0.00112 \\
\hline-0.00486 & -0.00018 & -0.0042 & 0.000135 & -0.00325 & 0.000442 & -0.00178 & $7.19 \mathrm{E}-05$ \\
\hline-0.00478 & -0.00115 & -0.00411 & -0.00138 & -0.00307 & -0.00132 & -0.00159 & -0.00118 \\
\hline-0.00429 & -0.00278 & -0.00376 & -0.00259 & -0.0028 & -0.00229 & -0.00144 & -0.002 \\
\hline-0.00363 & -0.0038 & -0.00326 & -0.00346 & -0.00243 & -0.00311 & -0.00124 & -0.00272 \\
\hline-0.00285 & -0.00452 & -0.00261 & -0.0042 & -0.00194 & -0.0038 & -0.00098 & -0.00333 \\
\hline-0.00193 & -0.00507 & -0.00183 & -0.00478 & -0.00136 & -0.00437 & -0.00066 & -0.00384 \\
\hline-0.00091 & -0.00541 & -0.00094 & -0.00518 & -0.0007 & -0.00477 & -0.0003 & -0.00421 \\
\hline 0.000172 & -0.00554 & $2.39 \mathrm{E}-05$ & -0.00538 & $2.96 \mathrm{E}-05$ & -0.00499 & $9.56 \mathrm{E}-05$ & -0.00442 \\
\hline 0.00128 & -0.00544 & 0.001027 & -0.00535 & 0.000795 & -0.00501 & 0.000518 & -0.00445 \\
\hline 0.002359 & -0.00509 & 0.002029 & -0.00507 & 0.001569 & -0.00479 & 0.000949 & -0.00428 \\
\hline 0.003357 & -0.0045 & 0.002982 & -0.00454 & 0.002317 & -0.00433 & 0.001366 & -0.00389 \\
\hline 0.004218 & -0.00369 & 0.00383 & -0.00374 & 0.003001 & -0.00361 & 0.001748 & -0.00327 \\
\hline 0.004892 & -0.00269 & 0.00451 & -0.0027 & 0.003572 & -0.00262 & 0.002071 & -0.00239 \\
\hline 0.005331 & -0.00155 & 0.004952 & -0.00145 & 0.003959 & -0.00134 & 0.0023 & -0.0012 \\
\hline 0.005499 & -0.00035 & 0.005082 & $-5.6 \mathrm{E}-05$ & 0.00404 & 0.000227 & 0.002346 & 0.000446 \\
\hline 0.005303 & 0.000211 & 0.004849 & 0.001298 & 0.003649 & 0.001839 & 0.001938 & 0.001773 \\
\hline 0.005074 & 0.000878 & 0.004466 & 0.001307 & 0.00335 & 0.001542 & 0.001879 & 0.001533 \\
\hline 0.004956 & 0.001295 & 0.004309 & 0.001642 & 0.003211 & 0.001817 & 0.001802 & 0.001781 \\
\hline
\end{tabular}


Table B10. Radial and tangential WSS values at Froude Number of 1.2, Stokes Number of 1 and Slope ratio of 0.1 .

\begin{tabular}{|c|c|c|c|c|c|c|c|}
\hline \multicolumn{2}{|c|}{$20 \%$} & \multicolumn{2}{|c|}{$40 \%$} & \multicolumn{2}{|c|}{$60 \%$} & \multicolumn{2}{|c|}{$80 \%$} \\
\hline Radial & Tangential & Radial & Tangential & Radial & Tangential & Radial & Tangential \\
\hline $\mathrm{Pa}$ & $\mathrm{Pa}$ & $\mathrm{Pa}$ & $\mathrm{Pa}$ & $\mathrm{Pa}$ & $\mathrm{Pa}$ & $\mathrm{Pa}$ & $\mathrm{Pa}$ \\
\hline 0.008801 & 0.013518 & 0.006922 & 0.013685 & 0.004625 & 0.013197 & 0.002309 & 0.012099 \\
\hline 0.006011 & 0.014866 & 0.004385 & 0.01472 & 0.002666 & 0.01399 & 0.001182 & 0.012717 \\
\hline 0.00308 & 0.015622 & 0.001793 & 0.015179 & 0.000705 & 0.014256 & $7.48 \mathrm{E}-05$ & 0.012868 \\
\hline 0.000125 & 0.015782 & -0.00075 & 0.015092 & -0.00119 & 0.014021 & -0.00097 & 0.012572 \\
\hline-0.00275 & 0.015379 & -0.00316 & 0.014481 & -0.00296 & 0.013315 & -0.00193 & 0.011867 \\
\hline-0.00545 & 0.014449 & -0.00537 & 0.013407 & -0.00454 & 0.012201 & -0.00278 & 0.010811 \\
\hline-0.00789 & 0.01305 & -0.0073 & 0.011931 & -0.0059 & 0.010737 & -0.00351 & 0.00946 \\
\hline-0.01001 & 0.011256 & -0.00891 & 0.010147 & -0.00701 & 0.009006 & -0.00411 & 0.007873 \\
\hline-0.01176 & 0.009135 & -0.01017 & 0.008137 & -0.00783 & 0.007093 & -0.00454 & 0.006126 \\
\hline-0.01311 & 0.006773 & -0.01109 & 0.006016 & -0.00835 & 0.005131 & -0.00481 & 0.004311 \\
\hline-0.01405 & 0.004232 & -0.01171 & 0.003867 & -0.00862 & 0.00332 & -0.00487 & 0.002697 \\
\hline-0.01456 & 0.001568 & -0.0121 & 0.001731 & -0.00887 & 0.001842 & -0.00518 & 0.002202 \\
\hline-0.01465 & -0.00116 & -0.01232 & -0.00045 & -0.00935 & 0.000404 & -0.00489 & -0.00012 \\
\hline-0.01429 & -0.00392 & -0.01226 & -0.00307 & -0.00887 & -0.00338 & -0.0045 & -0.00314 \\
\hline-0.01336 & -0.00718 & -0.01117 & -0.00708 & -0.00813 & -0.00636 & -0.00408 & -0.00563 \\
\hline-0.01143 & -0.01075 & -0.00981 & -0.00976 & -0.00704 & -0.00881 & -0.00345 & -0.00781 \\
\hline-0.00921 & -0.01309 & -0.00798 & -0.01199 & -0.00563 & -0.0109 & -0.00264 & -0.00964 \\
\hline-0.0066 & -0.01487 & -0.00578 & -0.01381 & -0.00395 & -0.01258 & -0.0017 & -0.0111 \\
\hline-0.00366 & -0.0161 & -0.00327 & -0.01512 & -0.00206 & -0.01381 & -0.00065 & -0.01216 \\
\hline 0.00049 & -0.01672 & -0.00056 & -0.01588 & $-3.7 \mathrm{E}-05$ & -0.01454 & 0.000454 & -0.01278 \\
\hline 0.002789 & -0.01669 & 0.002279 & -0.01604 & 0.002063 & -0.01473 & 0.001588 & -0.01294 \\
\hline 0.006061 & -0.01596 & 0.005142 & -0.01556 & 0.004168 & -0.01436 & 0.002703 & -0.01262 \\
\hline 0.00918 & -0.01453 & 0.007931 & -0.01441 & 0.006211 & -0.01341 & 0.003761 & -0.01179 \\
\hline 0.012003 & -0.01241 & 0.01054 & -0.01258 & 0.00812 & -0.01185 & 0.004716 & -0.01044 \\
\hline 0.014374 & -0.00965 & 0.012853 & -0.01004 & 0.009836 & -0.00968 & 0.005529 & -0.00858 \\
\hline 0.016133 & -0.00635 & 0.014731 & -0.00677 & 0.011306 & -0.00685 & 0.006193 & -0.00621 \\
\hline 0.017135 & -0.00265 & 0.015942 & -0.00271 & 0.012503 & -0.0031 & 0.006724 & -0.00325 \\
\hline 0.017214 & 0.001039 & 0.016077 & 0.002046 & 0.01302 & 0.002721 & 0.007509 & 0.001972 \\
\hline 0.016331 & 0.003014 & 0.014863 & 0.00487 & 0.011407 & 0.006093 & 0.006315 & 0.007073 \\
\hline 0.015238 & 0.006064 & 0.013414 & 0.00718 & 0.00991 & 0.007906 & 0.005378 & 0.007727 \\
\hline 0.013603 & 0.008986 & 0.011589 & 0.009815 & 0.008347 & 0.010029 & 0.004472 & 0.009469 \\
\hline 0.011469 & 0.011466 & 0.009449 & 0.01198 & 0.006608 & 0.011809 & 0.003469 & 0.010949 \\
\hline 0.010772 & 0.01209 & 0.008783 & 0.012511 & 0.006077 & 0.012244 & 0.003154 & 0.011307 \\
\hline
\end{tabular}


Table B11. Radial and tangential WSS values at Froude Number of 0.06, Stokes Number of 10 and Slope ratio of 0.1 .

\begin{tabular}{|c|c|c|c|c|c|c|c|}
\hline \multicolumn{2}{|c|}{$20 \%$} & \multicolumn{2}{|c|}{$40 \%$} & \multicolumn{2}{|c|}{$60 \%$} & \multicolumn{2}{|c|}{$80 \%$} \\
\hline Radial & Tangential & Radial & Tangential & Radial & Tangential & Radial & Tangential \\
\hline $\mathrm{Pa}$ & $\mathrm{Pa}$ & $\mathrm{Pa}$ & $\mathrm{Pa}$ & $\mathrm{Pa}$ & $\mathrm{Pa}$ & $\mathrm{Pa}$ & $\mathrm{Pa}$ \\
\hline-0.01247 & -0.00126 & -0.01011 & -0.00046 & -0.00716 & -0.00091 & -0.00203 & -0.00236 \\
\hline-0.01159 & -0.00427 & -0.00938 & -0.00333 & -0.00657 & -0.00349 & -0.00164 & -0.00469 \\
\hline-0.01045 & -0.00694 & -0.00845 & -0.00594 & -0.00584 & -0.00584 & -0.00127 & -0.00674 \\
\hline-0.00922 & -0.00888 & -0.00745 & -0.0079 & -0.00504 & -0.00759 & -0.00092 & -0.00818 \\
\hline-0.00781 & -0.01008 & -0.00629 & -0.00917 & -0.00414 & -0.00872 & -0.00055 & -0.00903 \\
\hline-0.0061 & -0.01083 & -0.00484 & -0.01003 & -0.00304 & -0.00947 & $-8.3 \mathrm{E}-05$ & -0.00953 \\
\hline-0.00357 & -0.01215 & -0.00271 & -0.01144 & -0.00144 & -0.01071 & 0.000534 & -0.01039 \\
\hline-0.00091 & -0.01317 & -0.00045 & -0.01257 & 0.00023 & -0.01166 & 0.001137 & -0.01097 \\
\hline 0.001648 & -0.01346 & 0.001738 & -0.01298 & 0.001843 & -0.01192 & 0.001684 & -0.01092 \\
\hline 0.004424 & -0.01286 & 0.004079 & -0.01247 & 0.003555 & -0.01133 & 0.002199 & -0.01003 \\
\hline 0.006455 & -0.0112 & 0.005744 & -0.01094 & 0.004723 & -0.00975 & 0.002412 & -0.00827 \\
\hline 0.008326 & -0.00908 & 0.007258 & -0.00891 & 0.005782 & -0.00774 & 0.00262 & -0.00614 \\
\hline 0.009816 & -0.00707 & 0.008415 & +0.00699 & 0.006527 & -0.00583 & 0.002626 & -0.00415 \\
\hline 0.011215 & -0.00516 & 0.009472 & -0.00515 & 0.007185 & -0.00402 & 0.002663 & -0.00229 \\
\hline 0.012262 & -0.00302 & 0.010201 & -0.00307 & 0.007575 & -0.00203 & 0.002588 & -0.00032 \\
\hline 0.012633 & -0.00037 & 0.01035 & -0.00052 & 0.007539 & 0.000335 & 0.00236 & 0.001897 \\
\hline 0.01225 & 0.002593 & 0.009861 & 0.002291 & 0.007033 & 0.002889 & 0.001947 & 0.004201 \\
\hline 0.011301 & 0.005514 & 0.008928 & 0.005062 & 0.00622 & 0.005333 & 0.001482 & 0.006326 \\
\hline 0.009999 & 0.007707 & 0.007739 & 0.00711 & 0.005262 & 0.007085 & 0.001013 & 0.00776 \\
\hline 0.008362 & 0.009674 & 0.006342 & 0.008956 & 0.004136 & 0.008601 & 0.000568 & 0.008865 \\
\hline 0.006722 & 0.010464 & 0.004952 & 0.009675 & 0.003104 & 0.009111 & 0.000158 & 0.009103 \\
\hline 0.004902 & 0.011028 & 0.003453 & 0.010196 & 0.002013 & 0.009433 & -0.00023 & 0.009163 \\
\hline 0.002647 & 0.012016 & 0.001634 & 0.011128 & 0.00071 & 0.010125 & -0.00067 & 0.009504 \\
\hline 0.000274 & 0.012872 & -0.00026 & 0.01197 & -0.0006 & 0.010728 & -0.00107 & 0.009778 \\
\hline-0.00236 & 0.013167 & -0.00233 & 0.012301 & -0.00205 & 0.010902 & -0.00152 & 0.009639 \\
\hline-0.00505 & 0.012412 & -0.00441 & 0.011666 & -0.00347 & 0.010241 & -0.0019 & 0.008746 \\
\hline-0.00673 & 0.010602 & -0.00568 & 0.010102 & -0.00431 & 0.00873 & -0.00198 & 0.007161 \\
\hline-0.00807 & 0.008536 & -0.00668 & 0.0083 & -0.00497 & 0.007042 & -0.00201 & 0.005429 \\
\hline-0.00951 & 0.006716 & -0.00779 & 0.006724 & -0.00571 & 0.005579 & -0.0021 & 0.003915 \\
\hline-0.01111 & 0.005078 & -0.00903 & 0.005308 & -0.00653 & 0.004289 & -0.00226 & 0.00258 \\
\hline-0.01233 & 0.003145 & -0.01 & 0.003591 & -0.00716 & 0.002742 & -0.00233 & 0.001047 \\
\hline-0.01271 & 0.000593 & -0.01031 & 0.001261 & -0.00733 & 0.000646 & -0.00221 & -0.00093 \\
\hline-0.01249 & -0.0013 & -0.01012 & -0.0005 & -0.00716 & -0.00094 & -0.00203 & -0.00238 \\
\hline
\end{tabular}


Table B12. Radial and tangential WSS values at Froude Number of 0.09, Stokes Number of 10 and Slope ratio of 0.1 .

\begin{tabular}{|c|c|c|c|c|c|c|c|}
\hline \multicolumn{2}{|c|}{$20 \%$} & \multicolumn{2}{|c|}{$40 \%$} & \multicolumn{2}{|c|}{$60 \%$} & \multicolumn{2}{|c|}{$80 \%$} \\
\hline Radial & Tangential & Radial & Tangential & Radial & Tangential & Radial & Tangential \\
\hline $\mathrm{Pa}$ & $\mathrm{Pa}$ & $\mathrm{Pa}_{\mathrm{a}}$ & $\mathrm{Pa}$ & $\mathrm{Pa}$ & $\mathrm{Pa}$ & $\mathrm{Pa}$ & $\mathrm{Pa}$ \\
\hline 0.015527 & -0.02337 & 0.013877 & -0.02299 & 0.011361 & -0.02058 & 0.005673 & -0.01641 \\
\hline 0.022651 & -0.01964 & 0.019801 & -0.01918 & 0.015399 & -0.01699 & 0.0071 & -0.01251 \\
\hline 0.028598 & -0.01392 & 0.024661 & -0.01339 & 0.018463 & -0.01159 & 0.007944 & -0.0072 \\
\hline 0.030438 & -0.00735 & 0.025944 & -0.00684 & 0.018886 & -0.00544 & 0.007479 & -0.0015 \\
\hline 0.028585 & -0.00092 & 0.024082 & -0.00052 & 0.017078 & 0.000449 & 0.0061 & 0.003735 \\
\hline 0.024969 & 0.004392 & 0.020749 & 0.004587 & 0.014315 & 0.005167 & 0.004448 & 0.007738 \\
\hline 0.022178 & 0.009019 & 0.018168 & 0.008968 & 0.012153 & 0.009125 & 0.003281 & 0.010896 \\
\hline 0.020952 & 0.013162 & 0.016966 & 0.012826 & 0.011064 & 0.012571 & 0.002758 & 0.013508 \\
\hline 0.020449 & 0.017384 & 0.016395 & 0.016746 & 0.010442 & 0.01605 & 0.002504 & 0.016083 \\
\hline 0.018931 & 0.021651 & 0.014949 & 0.020677 & 0.009205 & 0.019493 & 0.001945 & 0.01857 \\
\hline 0.015174 & 0.025164 & 0.01165 & 0.023839 & 0.006747 & 0.022177 & 0.000835 & 0.02037 \\
\hline 0.00885 & 0.027787 & 0.006321 & 0.026132 & 0.002921 & 0.024028 & -0.00085 & 0.021362 \\
\hline 0.001604 & 0.028746 & 0.000369 & 0.026903 & -0.00115 & 0.024488 & -0.00246 & 0.02118 \\
\hline-0.00495 & 0.028152 & -0.00492 & 0.026322 & -0.00467 & 0.023754 & -0.00366 & 0.020026 \\
\hline-0.00868 & 0.026297 & -0.00787 & 0.024741 & -0.00652 & 0.022031 & -0.00399 & 0.018217 \\
\hline-0.01147 & 0.024394 & -0.00999 & 0.023198 & -0.00784 & 0.020387 & -0.00405 & 0.016432 \\
\hline-0.01593 & 0.022329 & -0.01346 & 0.021444 & -0.01014 & 0.018675 & -0.00459 & 0.014481 \\
\hline-0.02306 & 0.018611 & -0.01915 & 0.017978 & -0.01393 & 0.01571 & -0.00599 & 0.011329 \\
\hline-0.02845 & 0.013088 & -0.0235 & 0.012833 & -0.01666 & 0.011233 & -0.00676 & 0.007042 \\
\hline-0.02976 & 0.006949 & -0.0246 & 0.007158 & -0.01716 & 0.006184 & -0.00647 & 0.002452 \\
\hline-0.0277 & 0.00117 & -0.02296 & 0.001788 & -0.0158 & 0.001327 & -0.00536 & -0.00181 \\
\hline-0.02455 & -0.00396 & -0.02042 & -0.00306 & -0.01381 & -0.0031 & -0.00415 & -0.00562 \\
\hline-0.02234 & -0.00853 & -0.0186 & -0.00744 & -0.01233 & -0.00716 & .0 .00331 & -0.00899 \\
\hline-0.02164 & -0.01284 & -0.01803 & -0.01162 & -0.01175 & -0.01103 & -0.00301 & -0.01211 \\
\hline-0.02142 & -0.0173 & -0.01788 & -0.01595 & -0.01149 & -0.01504 & -0.00287 & -0.01527 \\
\hline-0.01981 & -0.0219 & -0.01658 & -0.02049 & -0.01041 & -0.01925 & -0.00232 & -0.01856 \\
\hline-0.01573 & -0.02571 & -0.01311 & -0.02434 & -0.00784 & -0.02284 & -0.00111 & -0.0213 \\
\hline-0.00883 & -0.02863 & -0.00718 & -0.02738 & -0.00349 & -0.0257 & 0.000873 & -0.0233 \\
\hline-0.0011 & -0.02964 & -0.00047 & -0.02858 & 0.001341 & -0.02679 & 0.002926 & -0.02375 \\
\hline 0.005378 & -0.02884 & 0.005195 & -0.02799 & 0.005343 & -0.0261 & 0.004367 & -0.02263 \\
\hline 0.00887 & -0.0268 & 0.008278 & -0.02621 & 0.007438 & -0.02407 & 0.004745 & -0.02045 \\
\hline 0.012577 & -0.02478 & 0.011416 & -0.02435 & 0.009649 & -0.02202 & 0.005223 & -0.01807 \\
\hline 0.012577 & -0.02478 & 0.011416 & -0.02435 & 0.009649 & -0.02202 & 0.005223 & -0.01807 \\
\hline
\end{tabular}


Table B13. Radial and tangential WSS values at Froude Number of 1, Stokes Number of 10 and Slope ratio of 0.1.

\begin{tabular}{|c|c|c|c|c|c|c|c|}
\hline \multicolumn{2}{|c|}{$20 \%$} & \multicolumn{2}{|c|}{$40 \%$} & \multicolumn{2}{|c|}{$60 \%$} & \multicolumn{2}{|c|}{$80 \%$} \\
\hline $\begin{array}{c}\text { Radial } \\
\text { Pa }\end{array}$ & $\begin{array}{c}\text { Tangential } \\
\mathrm{Pa}\end{array}$ & $\begin{array}{c}\text { Radial } \\
\mathbf{P a}\end{array}$ & $\begin{array}{c}\text { Tangential } \\
\mathrm{Pa}\end{array}$ & $\begin{array}{c}\text { Radial } \\
\mathbf{P a}\end{array}$ & $\begin{array}{c}\text { Tangential } \\
\mathbf{P a}\end{array}$ & $\begin{array}{c}\text { Radial } \\
\mathbf{P a}\end{array}$ & $\begin{array}{c}\text { Tangential } \\
\mathrm{Pa}\end{array}$ \\
\hline-0.06882 & -0.20969 & -0.08642 & -0.21169 & -0.07781 & -0.22485 & -0.05691 & -0.2239 \\
\hline-0.01321 & -0.23523 & -0.03665 & -0.2411 & -0.04062 & -0.25794 & -0.03609 & -0.25543 \\
\hline 0.048491 & -0.24125 & 0.01992 & -0.24954 & 0.003539 & -0.27048 & -0.01111 & -0.2681 \\
\hline 0.112041 & -0.22559 & 0.078728 & -0.23406 & 0.050744 & -0.25891 & 0.015338 & -0.25849 \\
\hline 0.172676 & -0.18781 & 0.134647 & -0.19397 & 0.096379 & -0.22193 & 0.040561 & -0.22525 \\
\hline 0.225711 & -0.12913 & 0.18271 & -0.13084 & 0.135991 & -0.16097 & 0.061906 & -0.16981 \\
\hline 0.2668 & -0.05233 & 0.218474 & -0.04833 & 0.165669 & -0.08008 & 0.077315 & -0.09601 \\
\hline 0.292224 & 0.038294 & 0.238484 & 0.048018 & 0.182517 & 0.014529 & 0.085404 & -0.00987 \\
\hline 0.299003 & 0.137313 & 0.240552 & 0.151555 & 0.184785 & 0.115571 & 0.085419 & 0.081544 \\
\hline 0.285044 & 0.239232 & 0.223459 & 0.255543 & 0.171874 & 0.215602 & 0.077424 & 0.171229 \\
\hline 0.249483 & 0.337487 & 0.187443 & 0.352782 & 0.144419 & 0.307455 & 0.062032 & 0.25259 \\
\hline 0.193004 & 0.425161 & 0.134247 & 0.436467 & 0.104301 & 0.384552 & 0.040514 & 0.320106 \\
\hline 0.118165 & 0.495476 & 0.067023 & 0.500533 & 0.054407 & 0.441448 & 0.014297 & 0.369361 \\
\hline 0.029742 & 0.542512 & -0.00971 & 0.539964 & -0.00207 & 0.474595 & -0.01496 & 0.397487 \\
\hline-0.06637 & 0.562291 & -0.09105 & 0.551861 & -0.06157 & 0.482421 & -0.04555 & 0.403274 \\
\hline-0.16442 & 0.553199 & -0.17178 & 0.535649 & -0.12024 & 0.465428 & -0.07573 & 0.387405 \\
\hline-0.25769 & 0.51616 & -0.24697 & 0.493197 & -0.17477 & 0.42565 & -0.10396 & 0.351883 \\
\hline-0.34011 & 0.454033 & -0.31212 & 0.427802 & -0.22232 & 0.366092 & -0.12873 & 0.299305 \\
\hline-0.45427 & 0.271424 & -0.39941 & 0.245361 & -0.28779 & 0.201627 & -0.16308 & 0.154812 \\
\hline-0.47994 & 0.16056 & -0.41743 & 0.13741 & -0.30276 & 0.104399 & -0.17091 & 0.069473 \\
\hline-0.48259 & 0.043472 & -0.41701 & 0.024535 & -0.30484 & 0.002586 & -0.17188 & -0.01997 \\
\hline-0.46175 & -0.07431 & -0.39777 & -0.08818 & -0.2937 & -0.0996 & -0.16575 & -0.10985 \\
\hline-0.41782 & -0.18683 & -0.35995 & -0.19532 & -0.26946 & -0.19734 & -0.15244 & -0.19596 \\
\hline-0.35211 & -0.28768 & -0.30442 & -0.29107 & -0.2324 & -0.2855 & -0.13215 & -0.27355 \\
\hline-0.26706 & -0.37026 & -0.23298 & -0.36945 & -0.18372 & -0.35855 & -0.10532 & -0.33748 \\
\hline-0.16663 & -0.42823 & -0.14867 & -0.42455 & -0.12536 & -0.41101 & -0.07299 & -0.38309 \\
\hline-0.05629 & -0.45645 & -0.05597 & -0.45135 & -0.06013 & -0.43814 & -0.0368 & -0.4065 \\
\hline 0.056876 & -0.45173 & 0.039315 & -0.44648 & 0.008116 & -0.4367 & 0.000561 & -0.40494 \\
\hline 0.056876 & -0.45173 & 0.039315 & -0.44648 & 0.008116 & -0.4367 & 0.000561 & -0.40494 \\
\hline 0.165052 & -0.41369 & 0.130475 & -0.40937 & 0.074826 & -0.40584 & 0.036585 & -0.37738 \\
\hline 0.260581 & -0.34512 & 0.211039 & -0.34237 & 0.135177 & -0.34714 & 0.068666 & -0.32516 \\
\hline 0.337071 & -0.25145 & 0.275243 & -0.25047 & 0.184907 & -0.26454 & 0.094512 & -0.25224 \\
\hline 0.364237 & -0.20248 & 0.297814 & -0.20228 & 0.203051 & -0.22069 & 0.103813 & -0.21359 \\
\hline
\end{tabular}


Table B14. Radial and tangential WSS values at Froude Number of 2.8, Stokes Number of 10 and Slope ratio of 0.1 .

\begin{tabular}{|c|c|c|c|c|c|c|c|}
\hline \multicolumn{2}{|c|}{$20 \%$} & \multicolumn{2}{|c|}{$40 \%$} & \multicolumn{2}{|c|}{$60 \%$} & \multicolumn{2}{|c|}{$80 \%$} \\
\hline Radial & Tangential & Radial & Tangential & Radial & Tangential & Radial & Tangential \\
\hline $\mathrm{Pa}$ & $\mathrm{Pa}$ & $\mathrm{Pa}$ & $\mathrm{Pa}$ & $\mathrm{Pa}$ & $\mathrm{Pa}$ & $\mathrm{Pa}$ & $\mathrm{Pa}$ \\
\hline 1.958803 & 1.799803 & 1.568941 & 1.731196 & 0.978079 & 1.640386 & 0.228816 & 1.590798 \\
\hline 1.576536 & 2.165424 & 1.240585 & 2.066087 & 0.734734 & 1.936071 & 0.11448 & 1.799096 \\
\hline 1.135458 & 2.443255 & 0.868589 & 2.316847 & 0.467413 & 2.15295 & -0.00177 & 1.934604 \\
\hline 0.652344 & 2.627908 & 0.466651 & 2.47988 & 0.184946 & 2.288563 & -0.11786 & 1.997326 \\
\hline 0.146017 & 2.709883 & 0.050512 & 2.547845 & -0.10062 & 2.337663 & -0.22705 & 1.984814 \\
\hline-0.36403 & 2.688589 & -0.36468 & 2.522062 & -0.38044 & 2.302435 & -0.32741 & 1.902019 \\
\hline-0.861 & 2.56741 & -0.76678 & 2.405231 & -0.64653 & 2.185667 & -0.41669 & 1.752466 \\
\hline-1.32168 & 2.348431 & -1.13603 & 2.20045 & -0.88289 & 1.990525 & -0.48325 & 1.540793 \\
\hline-1.73528 & 2.042888 & -1.46725 & 1.917809 & -1.09149 & 1.726802 & -0.53727 & 1.277249 \\
\hline-2.08135 & 1.661197 & -1.74256 & 1.566033 & -1.25694 & 1.401797 & -0.56649 & 0.968938 \\
\hline-2.35377 & 1.212226 & -1.96001 & 1.151906 & -1.38304 & 1.020959 & -0.58164 & 0.621169 \\
\hline-2.53542 & 0.717962 & -2.10362 & 0.694787 & -1.45534 & 0.601576 & -0.56995 & 0.249499 \\
\hline-2.62297 & 0.195024 & -2.17201 & 0.208844 & -1.47647 & 0.155995 & -0.53968 & -0.1354 \\
\hline-2.60859 & -0.34057 & -2.15659 & -0.29223 & -1.43868 & -0.30367 & -0.48397 & -0.52348 \\
\hline-2.49681 & -0.86575 & -2.06286 & -0.78792 & -1.35036 & -0.75961 & -0.41494 & -0.89925 \\
\hline-2.28465 & -1.36041 & -1.88491 & -1.25903 & -1.20408 & -1.19362 & -0.32474 & -1.24694 \\
\hline-1.98246 & -1.80428 & -1.63133 & -1.68607 & -1.00835 & -1.58766 & -0.22096 & -1.55263 \\
\hline-1.5989 & -2.1802 & -1.30851 & -2.05136 & -0.76776 & -1.92424 & -0.10537 & -1.80159 \\
\hline-1.1494 & -2.46975 & -0.92905 & -2.33616 & -0.49214 & -2.18585 & 0.015978 & -1.98032 \\
\hline-0.65164 & -2.66358 & -0.50767 & -2.52962 & -0.19196 & -2.36138 & 0.139331 & -2.08053 \\
\hline-0.12295 & -2.74743 & -0.0591 & -2.61651 & 0.122411 & -2.43629 & 0.261761 & -2.09111 \\
\hline 0.409531 & -2.72108 & 0.391526 & -2.59511 & 0.431338 & -2.40824 & 0.370985 & -2.01253 \\
\hline 0.926557 & -2.58207 & 0.827619 & -2.46235 & 0.723813 & -2.27449 & 0.465566 & -1.84462 \\
\hline 1.407097 & -2.33778 & 1.230435 & -2.22543 & 0.987531 & -2.04288 & 0.543394 & -1.59633 \\
\hline 1.827858 & -1.99727 & 1.578482 & -1.89374 & 1.206389 & -1.72281 & 0.595681 & -1.27833 \\
\hline 2.174878 & -1.57177 & 1.860965 & -1.47944 & 1.376242 & -1.32698 & 0.626851 & -0.90376 \\
\hline 2.430296 & -1.08579 & 2.061798 & -1.00747 & 1.484916 & -0.87969 & 0.629383 & -0.49521 \\
\hline 2.586018 & -0.55689 & 2.175815 & -0.49634 & 1.531993 & -0.39932 & 0.607936 & -0.06955 \\
\hline 2.633891 & -0.00759 & 2.196339 & 0.030938 & 1.512217 & 0.091776 & 0.558057 & 0.353152 \\
\hline 2.578044 & 0.53683 & 2.130706 & 0.549111 & 1.435986 & 0.569407 & 0.491248 & 0.751812 \\
\hline 2.419696 & 1.05876 & 1.97959 & 1.041589 & 1.302786 & 1.019741 & 0.405646 & 1.116074 \\
\hline 2.164476 & 1.535438 & 1.749803 & 1.486646 & 1.118231 & 1.421745 & 0.302226 & 1.428964 \\
\hline 1.956702 & 1.807056 & 1.567576 & 1.737817 & 0.97784 & 1.646331 & 0.229376 & 1.596344 \\
\hline
\end{tabular}


Table B15. Radial and tangential WSS values at Froude Number of 5, Stokes Number of 10 and Slope ratio of 0.1 .

\begin{tabular}{|c|c|c|c|c|c|c|c|}
\hline \multicolumn{2}{|c|}{$20 \%$} & \multicolumn{2}{|c|}{$40 \%$} & \multicolumn{2}{|c|}{$60 \%$} & \multicolumn{2}{|c|}{$80 \%$} \\
\hline Radial & Tangential & Radial & Tangential & Radial & Tangential & Radial & Tangential \\
\hline $\mathrm{Pa}$ & $\mathrm{Pa}$ & $\mathrm{Pa}$ & $\mathrm{Pa}$ & $\mathrm{Pa}$ & $\mathrm{Pa}$ & $\mathrm{Pa}$ & $\mathrm{Pa}$ \\
\hline $5.3 \mathrm{E}-08$ & $-1.2 \mathrm{E}-07$ & $5.89 \mathrm{E}-08$ & $4.38 \mathrm{E}-08$ & $1.1 \mathrm{E}-07$ & $-1.2 \mathrm{E}-07$ & $9.01 \mathrm{E}-07$ & $1.25 \mathrm{E}-06$ \\
\hline $3.8 \mathrm{E}-09$ & $-1.4 \mathrm{E}-07$ & $6.68 \mathrm{E}-08$ & $6.26 \mathrm{E}-08$ & $8.73 \mathrm{E}-08$ & $-1.5 \mathrm{E}-07$ & $7.2 \mathrm{E}-07$ & $1.48 \mathrm{E}-06$ \\
\hline $2 \mathrm{E}-10$ & $-8.8 \mathrm{E}-08$ & $1.27 \mathrm{E}-07$ & $1.4 \mathrm{E}-07$ & $1.2 \mathrm{E}-07$ & $-1.2 \mathrm{E}-07$ & $5.87 \mathrm{E}-07$ & $1.71 \mathrm{E}-06$ \\
\hline$-6.9 \mathrm{E}-08$ & $-1.1 \mathrm{E}-07$ & $1.2 \mathrm{E}-07$ & $1.37 \mathrm{E}-07$ & 7.97E-08 & $-1.4 \mathrm{E}-07$ & $3.65 \mathrm{E}-07$ & $1.83 \mathrm{E}-06$ \\
\hline$-5.1 E-08$ & $-1.7 \mathrm{E}-07$ & $2.03 \mathrm{E}-07$ & $9.51 \mathrm{E}-08$ & $1.4 \mathrm{E}-07$ & $-1.7 \mathrm{E}-07$ & $2.55 \mathrm{E}-07$ & $1.87 \mathrm{E}-06$ \\
\hline$-9.7 \mathrm{E}-08$ & $-1.3 \mathrm{E}-07$ & $2.16 \mathrm{E}-07$ & $1.32 \mathrm{E}-07$ & $1.27 \mathrm{E}-07$ & $-1.3 \mathrm{E}-07$ & $6.98 \mathrm{E}-08$ & $1.9 \mathrm{E}-06$ \\
\hline$-1.2 \mathrm{E}-07$ & $-1.7 \mathrm{E}-07$ & $2.51 \mathrm{E}-07$ & $8.23 \mathrm{E}-08$ & $1.44 \mathrm{E}-07$ & $-1.4 \mathrm{E}-07$ & $-6.6 \mathrm{E}-08$ & $1.82 \mathrm{E}-06$ \\
\hline$-1.4 \mathrm{E}-07$ & $-1.3 \mathrm{E}-07$ & $2.72 \mathrm{E}-07$ & $1.1 \mathrm{E}-07$ & $1.4 \mathrm{E}-07$ & $-7.1 \mathrm{E}-08$ & $-2.2 \mathrm{E}-07$ & $1.75 \mathrm{E}-06$ \\
\hline$-2 E-07$ & $-1.4 \mathrm{E}-07$ & $2.48 \mathrm{E}-07$ & $7.2 \mathrm{E}-08$ & $1.01 \mathrm{E}-07$ & $-4.7 \mathrm{E}-08$ & $-3.8 \mathrm{E}-07$ & $1.57 \mathrm{E}-06$ \\
\hline$-1.7 \mathrm{E}-07$ & $-1.3 \mathrm{E}-07$ & $2.97 \mathrm{E}-07$ & $5.11 \mathrm{E}-08$ & $1.4 \mathrm{E}-07$ & $8.5 \mathrm{E}-09$ & $-4.3 \mathrm{E}-07$ & $1.38 \mathrm{E}-06$ \\
\hline$-2.2 \mathrm{E}-07$ & $-1.2 \mathrm{E}-07$ & $2.53 \mathrm{E}-07$ & $1.63 \mathrm{E}-08$ & $9 \mathrm{E}-08$ & $5.12 \mathrm{E}-08$ & $-5.5 \mathrm{E}-07$ & $1.11 \mathrm{E}-06$ \\
\hline$-2 \mathrm{E}-07$ & $-1.5 \mathrm{E}-07$ & $2.68 \mathrm{E}-07$ & $-5.3 E-08$ & $1.06 \mathrm{E}-07$ & $7.28 \mathrm{E}-08$ & $-5.6 \mathrm{E}-07$ & $8.02 \mathrm{E}-07$ \\
\hline$-2.3 \mathrm{E}-07$ & $-1.4 \mathrm{E}-07$ & $2.31 \mathrm{E}-07$ & $-8.9 E-08$ & $7.46 \mathrm{E}-08$ & $1.29 \mathrm{E}-07$ & $-5.8 \mathrm{E}-07$ & $5.02 \mathrm{E}-07$ \\
\hline$-2.2 \mathrm{E}-07$ & $-7.6 \mathrm{E}-08$ & $2.11 \mathrm{E}-07$ & $-7.5 \mathrm{E}-08$ & $6.16 \mathrm{E}-08$ & $2.2 \mathrm{E}-07$ & $-5.5 E-07$ & $2.2 \mathrm{E}-07$ \\
\hline$-2 \mathrm{E}-07$ & $-7.3 \mathrm{E}-08$ & $1.93 \mathrm{E}-07$ & $-1.2 \mathrm{E}-07$ & $6.4 \mathrm{E}-08$ & $2.56 \mathrm{E}-07$ & $-4.6 \mathrm{E}-07$ & $-1 \mathrm{E}-07$ \\
\hline$-1.7 \mathrm{E}-07$ & $-9 \mathrm{E}-08$ & $1.75 \mathrm{E}-07$ & $-1.8 \mathrm{E}-07$ & $6.63 \mathrm{E}-08$ & $2.75 \mathrm{E}-07$ & $-3.5 \mathrm{E}-07$ & $-4.1 E-07$ \\
\hline$-9.9 \mathrm{E}-08$ & $-1.9 \mathrm{E}-08$ & $1.89 \mathrm{E}-07$ & $-1.5 \mathrm{E}-07$ & $1.1 \mathrm{E}-07$ & $3.59 \mathrm{E}-07$ & $-1.6 \mathrm{E}-07$ & $-6.3 E-07$ \\
\hline$-7.3 \mathrm{E}-08$ & $-4.3 \mathrm{E}-08$ & $1.53 \mathrm{E}-07$ & $-2 \mathrm{E}-07$ & $1.01 \mathrm{E}-07$ & $3.58 \mathrm{E}-07$ & $-1.2 \mathrm{E}-08$ & $-8.7 \mathrm{E}-07$ \\
\hline$-5.1 \mathrm{E}-08$ & $3.5 \mathrm{E}-09$ & $1.1 \mathrm{E}-07$ & $-1.9 \mathrm{E}-07$ & $8.66 \mathrm{E}-08$ & $4.09 \mathrm{E}-07$ & $1.45 \mathrm{E}-07$ & $-1 E-06$ \\
\hline$-6.6 E-08$ & $1.7 \mathrm{E}-08$ & $2.64 \mathrm{E}-08$ & $-1.9 \mathrm{E}-07$ & $2.7 \mathrm{E}-08$ & $4.13 \mathrm{E}-07$ & $2.59 \mathrm{E}-07$ & $-1.1 \mathrm{E}-06$ \\
\hline$-3.7 \mathrm{E}-08$ & $-4.1 \mathrm{E}-08$ & $-9.8 \mathrm{E}-09$ & $-2.5 \mathrm{E}-07$ & $1.47 \mathrm{E}-08$ & $3.61 \mathrm{E}-07$ & $4.21 \mathrm{E}-07$ & $-1.2 \mathrm{E}-06$ \\
\hline $1.37 \mathrm{E}-08$ & $8.8 \mathrm{E}-09$ & $-1.5 \mathrm{E}-08$ & $-2.1 \mathrm{E}-07$ & $4.08 \mathrm{E}-08$ & $3.84 \mathrm{E}-07$ & $6.27 \mathrm{E}-07$ & $-1.2 \mathrm{E}-06$ \\
\hline $6.22 \mathrm{E}-08$ & $5.9 \mathrm{E}-09$ & $-1.5 \mathrm{E}-08$ & $-2 \mathrm{E}-07$ & $6.85 \mathrm{E}-08$ & $3.55 \mathrm{E}-07$ & $8.22 \mathrm{E}-07$ & $-1.1 \mathrm{E}-06$ \\
\hline $5.35 \mathrm{E}-08$ & $-2.3 \mathrm{E}-08$ & $-7 \mathrm{E}-08$ & $-2.1 \mathrm{E}-07$ & $2.83 \mathrm{E}-08$ & $2.96 \mathrm{E}-07$ & $9.15 \mathrm{E}-07$ & $-1 E-06$ \\
\hline $9.76 \mathrm{E}-08$ & $-3.7 \mathrm{E}-08$ & $-5.5 \mathrm{E}-08$ & $-2 \mathrm{E}-07$ & $6.14 \mathrm{E}-08$ & $2.46 \mathrm{E}-07$ & $1.07 \mathrm{E}-06$ & $-8.6 \mathrm{E}-07$ \\
\hline $1.22 \mathrm{E}-07$ & $-3.9 \mathrm{E}-08$ & $-5.1 \mathrm{E}-08$ & $-1.6 \mathrm{E}-07$ & $7.53 \mathrm{E}-08$ & $2.07 \mathrm{E}-07$ & $1.17 \mathrm{E}-06$ & $-6.3 E-07$ \\
\hline $1.28 \mathrm{E}-07$ & $-4.6 \mathrm{E}-08$ & $-5.3 \mathrm{E}-08$ & $-1.3 \mathrm{E}-07$ & $7.6 \mathrm{E}-08$ & $1.55 \mathrm{E}-07$ & $1.22 \mathrm{E}-06$ & $-3.6 \mathrm{E}-07$ \\
\hline $1.05 \mathrm{E}-07$ & $-1.9 \mathrm{E}-08$ & $-7.4 \mathrm{E}-08$ & $-6.1 \mathrm{E}-08$ & $4.76 \mathrm{E}-08$ & $1.33 \mathrm{E}-07$ & $1.2 \mathrm{E}-06$ & $-4.5 \mathrm{E}-08$ \\
\hline $1.22 \mathrm{E}-07$ & $-7.8 \mathrm{E}-08$ & $-3.7 \mathrm{E}-08$ & $-7 \mathrm{E}-08$ & $8.24 \mathrm{E}-08$ & $3.99 \mathrm{E}-08$ & $1.22 \mathrm{E}-06$ & $2.3 \mathrm{E}-07$ \\
\hline $9.5 \mathrm{E}-08$ & $-7.7 \mathrm{E}-08$ & $-3.7 \mathrm{E}-08$ & $-2.3 \mathrm{E}-08$ & $6.52 \mathrm{E}-08$ & $-4.2 \mathrm{E}-09$ & $1.14 \mathrm{E}-06$ & 5.47E-07 \\
\hline $1.01 \mathrm{E}-07$ & $-5.2 E-08$ & $1.06 \mathrm{E}-08$ & $4.57 \mathrm{E}-08$ & $9.57 \mathrm{E}-08$ & $-2.1 \mathrm{E}-08$ & $1.08 \mathrm{E}-06$ & $8.79 \mathrm{E}-07$ \\
\hline $2.42 \mathrm{E}-08$ & $-8.8 \mathrm{E}-08$ & $-2 \mathrm{E}-08$ & $5.61 \mathrm{E}-08$ & $3.85 \mathrm{E}-08$ & $-7.8 \mathrm{E}-08$ & $9.01 \mathrm{E}-07$ & $1.15 \mathrm{E}-06$ \\
\hline $7 \mathrm{E}-08$ & $-7.2 \mathrm{E}-08$ & $6.73 \mathrm{E}-08$ & $9.68 \mathrm{E}-08$ & $1.22 \mathrm{E}-07$ & $-7.8 \mathrm{E}-08$ & $9.11 \mathrm{E}-07$ & $1.34 \mathrm{E}-06$ \\
\hline
\end{tabular}




\section{B.3. WSS data as a function of Stokes Number at different combinations of constant}

\section{Froude Number and constant Slope ratio}

Table B16. Radial and tangential WSS values at Stokes Number of 1, Froude Number of

0.1 and Slope ratio of 0.1 .

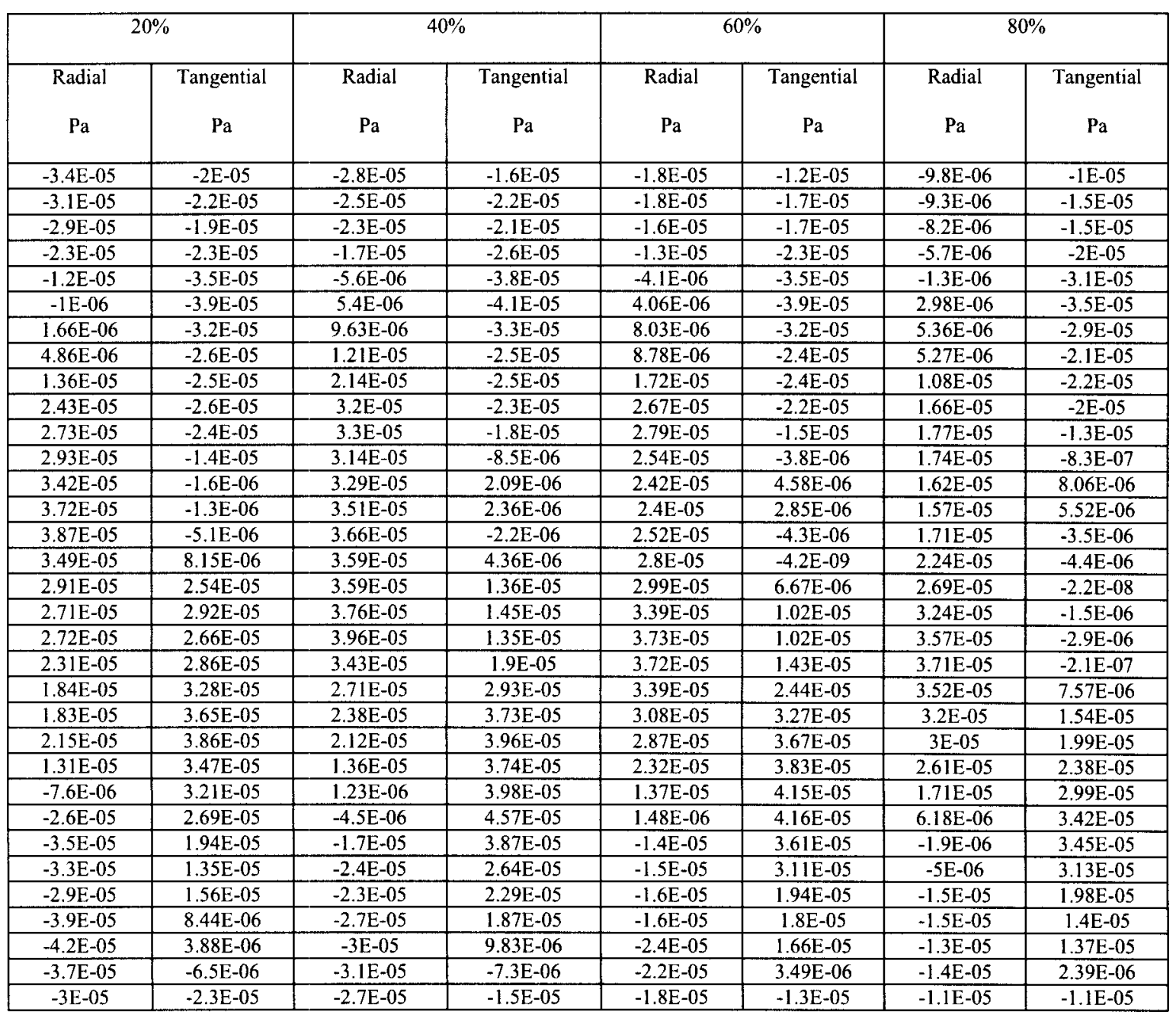


Table B17. Radial and tangential WSS values at Stokes Number of 5.2, Froude Number of 0.1 and Slope ratio of 0.1 .

\begin{tabular}{|c|c|c|c|c|c|c|c|}
\hline \multicolumn{2}{|c|}{$20 \%$} & \multicolumn{2}{|c|}{$40 \%$} & \multicolumn{2}{|c|}{$60 \%$} & \multicolumn{2}{|c|}{$80 \%$} \\
\hline $\begin{array}{c}\text { Radial } \\
\mathrm{Pa}\end{array}$ & $\begin{array}{c}\text { Tangential } \\
\mathrm{Pa}\end{array}$ & $\begin{array}{c}\text { Radial } \\
\mathrm{Pa}\end{array}$ & $\begin{array}{c}\text { Tangential } \\
\mathrm{Pa}\end{array}$ & $\begin{array}{c}\text { Radial } \\
\mathrm{Pa}\end{array}$ & $\begin{array}{c}\text { Tangential } \\
\mathrm{Pa}\end{array}$ & $\begin{array}{l}\text { Radial } \\
\mathrm{Pa}\end{array}$ & $\begin{array}{c}\text { Tangential } \\
\mathrm{Pa}\end{array}$ \\
\hline 0.000767 & 0.003678 & -0.00207 & 0.004543 & -0.00515 & 0.00501 & -0.00489 & 0.005109 \\
\hline 0.00049 & 0.002607 & -0.00249 & 0.003328 & -0.00562 & 0.003573 & -0.00548 & 0.003784 \\
\hline $5.85 \mathrm{E}-06$ & 0.002347 & -0.0029 & 0.002753 & -0.00594 & 0.00262 & -0.00611 & 0.002589 \\
\hline-0.00135 & 0.002446 & -0.00408 & 0.002601 & -0.00668 & 0.002067 & -0.00671 & 0.001535 \\
\hline-0.00224 & 0.002204 & -0.00453 & 0.002308 & -0.00663 & 0.001591 & -0.0065 & 0.000699 \\
\hline-0.00245 & 0.001194 & -0.00432 & 0.001491 & -0.00592 & 0.000905 & -0.00566 & $-4.7 \mathrm{E}-05$ \\
\hline-0.00272 & -0.00058 & -0.00447 & $4.08 \mathrm{E}-05$ & -0.00565 & $-5.6 \mathrm{E}-05$ & -0.00506 & -0.00063 \\
\hline-0.00243 & -0.00239 & -0.00422 & -0.0017 & -0.00532 & -0.00138 & -0.0046 & -0.00139 \\
\hline-0.00194 & -0.0037 & -0.00353 & -0.00345 & -0.00459 & -0.00338 & -0.00404 & -0.00352 \\
\hline-0.00173 & -0.00492 & -0.00319 & -0.0052 & -0.00409 & -0.00533 & -0.00343 & -0.00546 \\
\hline-0.00089 & -0.00631 & -0.00226 & -0.00677 & -0.00328 & -0.00654 & -0.0028 & -0.00612 \\
\hline 0.000874 & -0.00787 & -0.0004 & -0.00808 & -0.0019 & -0.00724 & -0.00214 & -0.00622 \\
\hline 0.002895 & -0.00922 & 0.001074 & -0.00895 & -0.00123 & -0.00754 & -0.00214 & -0.00603 \\
\hline 0.004953 & -0.0097 & 0.002375 & -0.00905 & -0.0008 & -0.0074 & -0.00239 & -0.0058 \\
\hline 0.006998 & -0.00915 & 0.003809 & -0.00832 & -0.00017 & -0.00673 & -0.00224 & -0.00544 \\
\hline 0.008427 & -0.00788 & 0.004538 & -0.0071 & $-3.8 \mathrm{E}-05$ & -0.00579 & -0.00212 & -0.00477 \\
\hline 0.00961 & -0.00639 & 0.005112 & -0.00582 & $1.42 \mathrm{E}-05$ & -0.00492 & -0.00219 & -0.004 \\
\hline 0.011034 & -0.00498 & 0.006276 & -0.00474 & 0.000721 & -0.00435 & -0.00222 & -0.00359 \\
\hline 0.012205 & -0.00352 & 0.007326 & -0.0036 & 0.001502 & -0.00374 & -0.0024 & -0.00351 \\
\hline 0.012916 & -0.00153 & 0.008032 & -0.00187 & 0.002216 & -0.00256 & -0.00223 & -0.00312 \\
\hline 0.013097 & 0.00089 & 0.008648 & 0.000515 & 0.003236 & -0.00054 & -0.00138 & -0.00174 \\
\hline 0.012587 & 0.003361 & 0.008554 & 0.003127 & 0.003721 & 0.002005 & -0.00059 & 0.000478 \\
\hline 0.011581 & 0.005358 & 0.007799 & 0.00532 & 0.003623 & 0.004469 & $-9.2 \mathrm{E}-05$ & 0.003069 \\
\hline 0.010609 & 0.006484 & 0.007145 & 0.006466 & 0.003372 & 0.00585 & $3.45 \mathrm{E}-05$ & 0.004829 \\
\hline 0.009618 & 0.006967 & 0.006414 & 0.006534 & 0.002944 & 0.005587 & $-4.5 \mathrm{E}-05$ & 0.00436 \\
\hline 0.00837 & 0.007116 & 0.005643 & 0.006215 & 0.002603 & 0.005031 & $3.96 \mathrm{E}-05$ & 0.003793 \\
\hline 0.007198 & 0.0071 & 0.005061 & 0.006145 & 0.00232 & 0.00526 & $3.66 \mathrm{E}-05$ & 0.004441 \\
\hline 0.005945 & 0.006942 & 0.004226 & 0.006332 & 0.001574 & 0.006002 & -0.00046 & 0.005652 \\
\hline 0.004606 & 0.00653 & 0.002972 & 0.006431 & 0.00025 & 0.006619 & -0.00148 & 0.006621 \\
\hline 0.003582 & 0.005765 & 0.001781 & 0.006192 & -0.00111 & 0.006741 & -0.00255 & 0.00687 \\
\hline 0.00274 & 0.004944 & 0.00064 & 0.005712 & -0.00241 & 0.006393 & -0.0034 & 0.006459 \\
\hline 0.001757 & 0.004282 & -0.00073 & 0.005163 & -0.00383 & 0.005795 & -0.00416 & 0.00585 \\
\hline 0.001325 & 0.004746 & -0.00115 & 0.005613 & -0.00415 & 0.006151 & -0.00424 & 0.00609 \\
\hline
\end{tabular}


Table B18. Radial and tangential WSS values at Stokes Number of 10, Froude Number of

\section{1 and Slope ratio of 0.1 .}

\begin{tabular}{|c|c|c|c|c|c|c|c|}
\hline \multicolumn{2}{|c|}{$20 \%$} & \multicolumn{2}{|c|}{$40 \%$} & \multicolumn{2}{|c|}{$60 \%$} & \multicolumn{2}{|c|}{$80 \%$} \\
\hline $\begin{array}{c}\text { Radial } \\
\mathrm{Pa}\end{array}$ & $\begin{array}{c}\text { Tangential } \\
\mathbf{P a}\end{array}$ & $\begin{array}{l}\text { Radial } \\
\qquad \mathrm{Pa}\end{array}$ & $\begin{array}{c}\text { Tangential } \\
\mathrm{Pa}\end{array}$ & $\begin{array}{l}\text { Radial } \\
\qquad \mathrm{Pa}\end{array}$ & $\begin{array}{c}\text { Tangential } \\
\text { Pa }\end{array}$ & $\begin{array}{l}\text { Radial } \\
\qquad \mathbf{P a}\end{array}$ & $\begin{array}{c}\text { Tangential } \\
\mathrm{Pa}\end{array}$ \\
\hline 0.01369 & 0.033598 & 0.010024 & 0.033265 & 0.00556 & 0.029057 & -0.00102 & 0.0268 \\
\hline 0.007219 & 0.035645 & 0.004517 & 0.034855 & 0.001764 & 0.030375 & -0.00238 & 0.027221 \\
\hline 0.000511 & 0.036345 & -0.00109 & 0.035179 & -0.00203 & 0.030634 & -0.00362 & 0.026706 \\
\hline-0.00618 & 0.035691 & -0.00662 & 0.034241 & -0.00572 & 0.029853 & -0.00471 & 0.025339 \\
\hline-0.01265 & 0.03379 & -0.01188 & 0.032193 & -0.00918 & 0.028143 & -0.00563 & 0.023196 \\
\hline-0.01868 & 0.030666 & -0.01675 & 0.029029 & -0.01233 & 0.025508 & -0.00635 & 0.020303 \\
\hline-0.02406 & 0.026461 & -0.02107 & 0.024914 & -0.01508 & 0.022062 & -0.00687 & 0.01678 \\
\hline-0.02858 & 0.02121 & -0.02469 & 0.019854 & -0.01735 & 0.017805 & -0.00715 & 0.012639 \\
\hline-0.03203 & 0.015117 & -0.02745 & 0.014028 & -0.01901 & 0.012863 & -0.00715 & 0.008011 \\
\hline-0.03433 & 0.008379 & -0.0293 & 0.007601 & -0.0201 & 0.007359 & -0.007 & 0.002997 \\
\hline-0.03531 & 0.001244 & -0.03006 & 0.000781 & -0.02044 & 0.001441 & -0.00655 & -0.00228 \\
\hline-0.03496 & -0.00599 & -0.02973 & -0.00617 & -0.02004 & -0.00468 & -0.00589 & -0.00762 \\
\hline-0.03323 & -0.01304 & -0.02824 & -0.01302 & -0.01886 & -0.01079 & -0.00495 & -0.01286 \\
\hline-0.03023 & -0.01967 & -0.02564 & -0.01947 & -0.01689 & -0.01664 & -0.00378 & -0.01779 \\
\hline-0.02602 & -0.02557 & -0.02199 & -0.02529 & -0.0142 & -0.02202 & -0.00241 & -0.02217 \\
\hline-0.02069 & -0.03048 & -0.01734 & -0.03016 & -0.01081 & -0.0266 & -0.00087 & -0.02577 \\
\hline-0.01449 & -0.03424 & -0.01187 & -0.03392 & -0.00686 & -0.03019 & 0.000789 & -0.0284 \\
\hline-0.0076 & -0.03667 & -0.00576 & -0.03632 & -0.00246 & -0.03256 & 0.002505 & -0.02985 \\
\hline-0.00032 & -0.03755 & 0.000717 & -0.03716 & 0.00217 & -0.03347 & 0.004181 & -0.0299 \\
\hline 0.006956 & -0.03688 & 0.007173 & -0.03636 & 0.006717 & -0.03285 & 0.005632 & -0.0286 \\
\hline 0.013991 & -0.0346 & 0.013351 & -0.0339 & 0.011019 & -0.03066 & 0.006906 & -0.02589 \\
\hline 0.02039 & -0.03086 & 0.018917 & -0.02992 & 0.014805 & -0.02706 & 0.007852 & -0.02196 \\
\hline 0.025894 & -0.02581 & 0.023619 & -0.02461 & 0.017916 & -0.0222 & 0.008442 & -0.01698 \\
\hline 0.03031 & -0.01971 & 0.02725 & -0.01825 & 0.02018 & -0.01637 & 0.008623 & -0.01126 \\
\hline 0.033409 & -0.01286 & 0.029609 & -0.01118 & 0.021463 & -0.00993 & 0.008369 & -0.00516 \\
\hline 0.035126 & -0.00554 & 0.030678 & -0.00375 & 0.021796 & -0.00322 & 0.00776 & 0.000992 \\
\hline 0.035387 & 0.001926 & 0.030435 & 0.003706 & 0.021207 & 0.00346 & 0.006884 & 0.006914 \\
\hline 0.034243 & 0.009215 & 0.028966 & 0.010876 & 0.019794 & 0.009817 & 0.005739 & 0.012366 \\
\hline 0.031694 & 0.016095 & 0.026322 & 0.01745 & 0.01756 & 0.015557 & 0.004359 & 0.017115 \\
\hline 0.027933 & 0.022293 & 0.022715 & 0.023237 & 0.014753 & 0.020564 & 0.002908 & 0.021036 \\
\hline 0.023119 & 0.027496 & 0.01831 & 0.027974 & 0.011476 & 0.024593 & 0.001398 & 0.02398 \\
\hline 0.017468 & 0.031647 & 0.013301 & 0.031615 & 0.007868 & 0.027673 & -0.00012 & 0.026018 \\
\hline 0.011229 & 0.034523 & 0.007916 & 0.034006 & 0.004099 & 0.029672 & -0.00156 & 0.027052 \\
\hline
\end{tabular}


Table B19. Radial and tangential WSS values at Stokes Number of 1, Froude Number of

\section{2 and Slope ratio of 0.1 .}

\begin{tabular}{|c|c|c|c|c|c|c|c|}
\hline \multicolumn{2}{|c|}{$20 \%$} & \multicolumn{2}{|c|}{$40 \%$} & \multicolumn{2}{|c|}{$60 \%$} & \multicolumn{2}{|c|}{$80 \%$} \\
\hline Radial & Tangential & Radial & Tangential & Radial & Tangential & Radial & Tangential \\
\hline $\mathrm{Pa}$ & $\mathrm{Pa}$ & $\mathrm{Pa}$ & $\mathrm{Pa}$ & $\mathrm{Pa}$ & $\mathrm{Pa}$ & $\mathrm{Pa}$ & $\mathrm{Pa}$ \\
\hline 0.010308 & 0.008905 & 0.008469 & 0.00998 & 0.005826 & 0.010156 & 0.00303 & 0.009515 \\
\hline 0.008189 & 0.010591 & 0.006328 & 0.011154 & 0.004177 & 0.010873 & 0.002145 & 0.00997 \\
\hline 0.005881 & 0.011767 & 0.004186 & 0.011866 & 0.002577 & 0.011272 & 0.001279 & 0.010212 \\
\hline 0.003492 & 0.012444 & 0.002077 & 0.012143 & 0.001026 & 0.011343 & 0.000424 & 0.010197 \\
\hline 0.001104 & 0.012643 & $5.4 \mathrm{E}-05$ & 0.012019 & -0.00046 & 0.011083 & -0.0004 & 0.009908 \\
\hline-0.0012 & 0.012394 & -0.00184 & 0.01152 & -0.00184 & 0.010503 & -0.00118 & 0.009342 \\
\hline-0.00335 & 0.011746 & -0.00356 & 0.010687 & -0.00308 & 0.009626 & -0.00188 & 0.008511 \\
\hline-0.00529 & 0.01075 & -0.00505 & 0.009574 & -0.00414 & 0.008494 & -0.00248 & 0.007446 \\
\hline-0.00698 & 0.009466 & -0.0063 & 0.00824 & -0.00499 & 0.007168 & -0.00296 & 0.006201 \\
\hline-0.0084 & 0.007949 & -0.00726 & 0.006763 & -0.00561 & 0.005719 & -0.00327 & 0.004829 \\
\hline-0.00953 & 0.006263 & -0.00796 & 0.005232 & -0.00597 & 0.004264 & -0.0034 & 0.003439 \\
\hline-0.01038 & 0.004457 & -0.00844 & 0.003731 & -0.00612 & 0.002975 & -0.00335 & 0.002262 \\
\hline-0.01095 & 0.002572 & -0.00877 & 0.002303 & -0.00625 & 0.002015 & -0.00337 & 0.001893 \\
\hline-0.01125 & 0.000624 & -0.00904 & 0.000928 & -0.0066 & 0.001279 & -0.00417 & 0.001966 \\
\hline-0.01128 & -0.00136 & -0.00927 & -0.00049 & -0.00719 & 0.000438 & -0.00529 & 0.001507 \\
\hline-0.01102 & -0.00339 & -0.00943 & -0.00203 & -0.00783 & -0.0007 & -0.00464 & -0.00059 \\
\hline-0.01044 & -0.00542 & -0.00942 & -0.00374 & -0.00781 & -0.00269 & -0.00413 & -0.00269 \\
\hline-0.0095 & -0.00742 & -0.00913 & -0.00565 & -0.00693 & -0.00521 & -0.00349 & -0.00478 \\
\hline-0.00818 & -0.00933 & -0.00804 & -0.00799 & -0.0058 & -0.00744 & -0.00274 & -0.00669 \\
\hline-0.00644 & -0.01106 & -0.00636 & -0.01014 & -0.00436 & -0.00938 & -0.00187 & -0.00833 \\
\hline-0.00428 & -0.01249 & -0.0043 & -0.01189 & -0.00274 & -0.01094 & -0.0009 & -0.00965 \\
\hline-0.00173 & -0.01349 & -0.00198 & -0.01312 & -0.00095 & -0.01207 & 0.000113 & -0.01061 \\
\hline 0.001123 & -0.01395 & 0.000545 & -0.01382 & 0.000931 & -0.01274 & 0.001151 & -0.01118 \\
\hline 0.004141 & -0.01371 & 0.003208 & -0.01396 & 0.002864 & -0.01294 & 0.002187 & -0.01135 \\
\hline 0.007123 & -0.01271 & 0.005941 & -0.01346 & 0.004797 & -0.01263 & 0.003189 & -0.01109 \\
\hline 0.009835 & -0.01094 & 0.008707 & -0.01223 & 0.006701 & -0.01179 & 0.004136 & -0.01043 \\
\hline 0.012049 & -0.00849 & 0.011478 & -0.01006 & 0.008584 & -0.01038 & 0.005012 & -0.00932 \\
\hline 0.013601 & -0.00553 & 0.014167 & -0.00631 & 0.010602 & -0.00814 & 0.005839 & -0.00776 \\
\hline 0.014358 & -0.00232 & 0.015194 & -0.0012 & 0.01395 & -0.001 & 0.006833 & -0.00544 \\
\hline 0.01432 & 0.000907 & 0.014283 & 0.002766 & 0.012 & 0.005195 & 0.00706 & 0.00866 \\
\hline 0.013563 & 0.003924 & 0.01264 & 0.005833 & 0.00965 & 0.007602 & 0.005137 & 0.008402 \\
\hline 0.012217 & 0.006582 & 0.010679 & 0.008172 & 0.007672 & 0.00906 & 0.004017 & 0.008922 \\
\hline 0.012188 & 0.006626 & 0.010641 & 0.008209 & 0.007638 & 0.009082 & 0.003999 & 0.00893 \\
\hline
\end{tabular}


Table B20. Radial and tangential WSS values at Stokes Number of 3.2, Froude Number of 1.2 and Slope ratio of 0.1 .

\begin{tabular}{|c|c|c|c|c|c|c|c|}
\hline \multicolumn{2}{|c|}{$20 \%$} & \multicolumn{2}{|c|}{$40 \%$} & \multicolumn{2}{|c|}{$60 \%$} & \multicolumn{2}{|c|}{$80 \%$} \\
\hline Radial & Tangential & Radial & Tangential & Radial & Tangential & Radial & Tangential \\
\hline $\mathrm{Pa}$ & $\mathrm{Pa}$ & $\mathrm{Pa}$ & $\mathrm{Pa}$ & $\mathrm{Pa}$ & $\mathrm{Pa}$ & $\mathrm{Pa}$ & $\mathrm{Pa}$ \\
\hline-0.06216 & 0.414674 & -0.09729 & 0.391686 & -0.10146 & 0.353023 & -0.03885 & 0.290887 \\
\hline 0.475729 & -0.17534 & 0.415942 & -0.19594 & 0.360088 & -0.21376 & 0.237 & -0.20766 \\
\hline 0.502674 & -0.05823 & 0.450777 & -0.05581 & 0.378687 & -0.05128 & 0.261374 & -0.01744 \\
\hline 0.50238 & 0.061275 & 0.444857 & 0.09186 & 0.358058 & 0.121669 & 0.218477 & 0.160004 \\
\hline 0.473524 & 0.173964 & 0.401369 & 0.225924 & 0.2952 & 0.27484 & 0.164941 & 0.304921 \\
\hline 0.422275 & 0.267132 & 0.330768 & 0.318549 & 0.211923 & 0.356269 & 0.111893 & 0.36303 \\
\hline 0.357434 & 0.334659 & 0.260709 & 0.354501 & 0.149788 & 0.338943 & 0.062396 & 0.313864 \\
\hline 0.287356 & 0.375743 & 0.200035 & 0.376476 & 0.11435 & 0.346557 & 0.044614 & 0.288362 \\
\hline 0.214568 & 0.404933 & 0.136757 & 0.396497 & 0.075889 & 0.367184 & 0.045605 & 0.322173 \\
\hline 0.137776 & 0.421902 & 0.072315 & 0.409595 & 0.027465 & 0.388107 & 0.00596 & 0.370072 \\
\hline 0.059237 & 0.428777 & 0.004552 & 0.412588 & -0.03457 & 0.395753 & -0.03114 & 0.360515 \\
\hline-0.01806 & 0.424164 & -0.06129 & 0.402921 & -0.08269 & 0.368193 & -0.0364 & 0.301578 \\
\hline-0.08836 & 0.408443 & -0.11818 & 0.384336 & -0.11247 & 0.348504 & -0.04023 & 0.294111 \\
\hline-0.15905 & 0.383353 & -0.17422 & 0.357989 & -0.15028 & 0.329296 & -0.05421 & 0.289341 \\
\hline-0.22353 & 0.345925 & -0.2245 & 0.321894 & -0.18528 & 0.294947 & -0.07515 & 0.26289 \\
\hline-0.2797 & 0.300452 & -0.26841 & 0.278306 & -0.21967 & 0.256559 & -0.08384 & 0.230087 \\
\hline-0.2797 & 0.300452 & -0.26841 & 0.278306 & -0.21967 & 0.256559 & -0.08384 & 0.230087 \\
\hline-0.32687 & 0.24653 & -0.30349 & 0.227815 & -0.2452 & 0.20895 & -0.09565 & 0.201616 \\
\hline-0.36322 & 0.184623 & -0.32965 & 0.172214 & -0.2649 & 0.162319 & -0.12801 & 0.163047 \\
\hline-0.38889 & 0.118596 & -0.34702 & 0.113263 & -0.27934 & 0.104218 & -0.15246 & 0.10252 \\
\hline-0.4038 & 0.048279 & -0.35374 & 0.053573 & -0.27832 & 0.04671 & -0.15532 & 0.034886 \\
\hline-0.40778 & -0.0261 & -0.35262 & -0.00374 & -0.26735 & -0.00263 & -0.14329 & -0.01153 \\
\hline-0.40138 & -0.10823 & -0.34832 & -0.06284 & -0.25675 & -0.03477 & -0.13481 & -0.02167 \\
\hline-0.38049 & -0.19982 & -0.34243 & -0.14588 & -0.26058 & -0.08652 & -0.13905 & -0.04411 \\
\hline-0.28089 & -0.36985 & -0.27203 & -0.32154 & -0.22981 & -0.2821 & -0.15156 & -0.25067 \\
\hline-0.20721 & -0.42959 & -0.21624 & -0.38616 & -0.18344 & -0.33816 & -0.11604 & -0.30792 \\
\hline-0.12309 & -0.47189 & -0.14771 & -0.4305 & -0.1342 & -0.38065 & -0.08597 & -0.32545 \\
\hline-0.03077 & -0.49421 & -0.07532 & -0.45435 & -0.08821 & -0.38937 & -0.05932 & -0.33945 \\
\hline 0.066812 & -0.49644 & -0.00069 & -0.4693 & -0.04537 & -0.4093 & -0.00827 & -0.38771 \\
\hline 0.166305 & -0.47658 & 0.082858 & -0.46937 & 0.028374 & -0.44947 & 0.025619 & -0.42389 \\
\hline 0.261588 & -0.43348 & 0.174974 & -0.44701 & 0.130894 & -0.45398 & 0.065652 & -0.43365 \\
\hline 0.348593 & -0.36809 & 0.267778 & -0.39468 & 0.222865 & -0.40474 & 0.122077 & -0.40745 \\
\hline-0.06715 & 0.415131 & -0.10093 & 0.391499 & -0.10324 & 0.354465 & -0.03927 & 0.295717 \\
\hline
\end{tabular}


Table B21. Radial and tangential WSS values at Stokes Number of 10, Froude Number of

\section{2 and Slope ratio of 0.1 .}

\begin{tabular}{|c|c|c|c|c|c|c|c|}
\hline \multicolumn{2}{|c|}{$20 \%$} & \multicolumn{2}{|c|}{$40 \%$} & \multicolumn{2}{|c|}{$60 \%$} & \multicolumn{2}{|c|}{$80 \%$} \\
\hline $\begin{array}{c}\text { Radial } \\
\mathrm{Pa}\end{array}$ & $\begin{array}{c}\text { Tangential } \\
\mathrm{Pa}\end{array}$ & $\begin{array}{c}\text { Radial } \\
\mathrm{Pa}\end{array}$ & $\begin{array}{c}\text { Tangential } \\
\mathrm{Pa}\end{array}$ & $\begin{array}{c}\text { Radial } \\
\mathrm{Pa}\end{array}$ & $\begin{array}{c}\text { Tangential } \\
\mathrm{Pa}\end{array}$ & $\begin{array}{c}\text { Radial } \\
\mathrm{Pa}\end{array}$ & $\begin{array}{c}\text { Tangential } \\
\mathrm{Pa}\end{array}$ \\
\hline 0.506326 & 10.91451 & -0.06423 & 10.27289 & -0.59523 & 9.435495 & -0.97628 & 8.006132 \\
\hline-1.51988 & 10.80252 & -1.70167 & 10.0839 & -1.69475 & 9.174437 & -1.35392 & 7.538684 \\
\hline-3.47262 & 10.29858 & -3.25105 & 9.558379 & -2.70695 & 8.625292 & -1.6717 & 6.844497 \\
\hline-5.28867 & 9.425603 & -4.66815 & 8.718564 & -3.60268 & 7.811168 & -1.91411 & 5.959479 \\
\hline-6.90803 & 8.228089 & -5.91736 & 7.611007 & -4.36818 & 6.775373 & -2.08709 & 4.920258 \\
\hline-6.90803 & 8.228089 & -5.91736 & 7.611007 & -4.36818 & 6.775373 & -2.08709 & 4.920258 \\
\hline-9.36302 & 5.035386 & -7.79826 & 4.732988 & -5.45051 & 4.151641 & -2.22158 & 2.491082 \\
\hline-10.1151 & 3.135339 & -8.37442 & 3.030899 & -5.73556 & 2.616264 & -2.17199 & 1.14375 \\
\hline-10.5206 & 1.116742 & -8.6909 & 1.215652 & -5.84946 & 0.981039 & -2.06222 & -0.2511 \\
\hline-10.5535 & -0.9591 & -8.72293 & -0.66839 & -5.7726 & -0.7196 & -1.88078 & -1.66599 \\
\hline-10.2141 & -3.01637 & -8.46958 & -2.56357 & -5.51274 & -2.43781 & -1.65168 & -3.06163 \\
\hline-9.4923 & -4.98788 & -7.91078 & -4.40896 & -5.05443 & -4.12305 & -1.34564 & $-4,40439$ \\
\hline-8.40475 & -6.80397 & -7.05617 & -6.1528 & -4.40468 & -5.72682 & -0.9955 & -5.64905 \\
\hline-6.98045 & -8.38355 & -5.91432 & -7.70644 & -3.57071 & -7.17015 & -0.5918 & -6.73742 \\
\hline-5.26641 & -9.6653 & -4.51493 & -9.01165 & -2.56912 & -8.39779 & -0.14781 & -7.62504 \\
\hline-3.33068 & -10.5748 & -2.90869 & -9.98326 & -1.43847 & -9.32473 & 0.313866 & -8.24576 \\
\hline-1.23283 & -11.069 & -1.13661 & -10.5662 & -0.1982 & -9.89494 & 0.798743 & -8.55333 \\
\hline 0.945325 & -11.1051 & 0.729913 & -10.7004 & 1.097071 & -10.0467 & 1.27778 & -8.49573 \\
\hline 3.10096 & -10.6784 & 2.595762 & -10.3685 & 2.383911 & -9.74713 & 1.726654 & -8.05631 \\
\hline 5.148582 & -9.79359 & 4.384305 & -9.56162 & 3.612575 & -8.98563 & 2.132176 & -7.22053 \\
\hline 6.981501 & -8.48259 & 5.989537 & -8.30221 & 4.696211 & -7.78012 & 2.459266 & -6.01165 \\
\hline 8.515936 & -6.80301 & 7.325743 & -6.64523 & 5.575972 & -6.17787 & 2.68763 & -4.47417 \\
\hline 9.681489 & -4.82091 & 8.324647 & -4.66646 & 6.20246 & -4.26043 & 2.800108 & -2.68797 \\
\hline 10.42239 & -2.62884 & 8.928847 & -2.47146 & 6.53071 & -2.13635 & 2.779189 & -0.75797 \\
\hline 10.70446 & -0.32803 & 9.10523 & -0.17104 & 6.534903 & 0.08191 & 2.617803 & 1.210887 \\
\hline 10.5272 & 1.963974 & 8.860597 & 2.099016 & 6.227155 & 2.246674 & 2.323196 & 3.082889 \\
\hline 9.905475 & 4.149556 & 8.217701 & 4.223618 & 5.636469 & 4.242382 & 1.927992 & 4.739663 \\
\hline 8.864768 & 6.141297 & 7.210559 & 6.126118 & 4.785147 & 5.995629 & 1.430845 & 6.131568 \\
\hline 7.463931 & 7.859957 & 5.911291 & 7.721568 & 3.748992 & 7.425345 & 0.890856 & 7.1858 \\
\hline 5.778123 & 9.235003 & 4.399592 & 8.950724 & 2.591612 & 8.48192 & 0.334692 & 7.872923 \\
\hline 3.887076 & 10.22581 & 2.757108 & 9.78972 & 1.382063 & 9.157147 & -0.19967 & 8.202507 \\
\hline 1.880102 & 10.81261 & 1.065275 & 10.23978 & 0.18174 & 9.468566 & -0.68242 & 8.209214 \\
\hline 1.134367 & 10.92633 & 0.447494 & 10.31084 & -0.24755 & 9.497421 & -0.84884 & 8.138956 \\
\hline
\end{tabular}


APPENDIX - C

\section{RELATIVE GENE EXPRESSION DATA FOR ATHEROGENIC GENES IN HUVECS UNDER SHEAR EXPOSURE AT 100 RPM AT THE MRNA LEVEL OBTAINED BY REAL TIME PCR ANALYSIS}

Table C1. Relative fold change of E-Selectin expression in HUVECs in and without the presence of $\mathrm{LTB}_{4}$ and LPS with or without shear exposure.

\begin{tabular}{|c|c|c|c|c|c|c|c|}
\hline \multicolumn{2}{|c|}{ HUVECs condition } & \multicolumn{3}{|c|}{ At $20 \%$ radial location } & \multicolumn{3}{|c|}{ At $85 \%$ radial location } \\
\hline \multirow{4}{*}{$\begin{array}{l}\text { Without } \\
\text { shear } \\
\text { exposure }\end{array}$} & Control & 1 & 1 & 1 & 1 & 1 & 1 \\
\hline & with $\mathrm{LTB}_{4}$ & 1.11161 & 1.556598 & 0.286454 & 1.11161 & 1.556598478 & 0.286454 \\
\hline & with LPS & 803.4586 & 1172.373 & 975.1682 & 803.4586 & 1172.372964 & 975.1682 \\
\hline & $\begin{array}{l}\text { with } \mathrm{LTB}_{4} \\
\text { and LPS }\end{array}$ & 698.463 & 938.7846 & 358.0735 & 698.463 & 938.7846 & 358.0735 \\
\hline \multirow{4}{*}{ 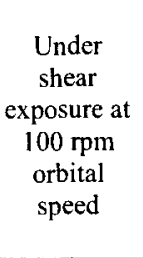 } & No treatment & 0.668896 & 1.259258 & 1.281959 & 0.417794 & 0.763395861 & 0.329412 \\
\hline & with $\mathrm{LTB}_{4}$ & 7.97447 & 8.359546 & 3.159121 & 3.519575 & 6.935233701 & 0.636438 \\
\hline & with LPS & 972.077 & 848.721 & 855.9929 & 656.8271 & 776.5932185 & 648.2202 \\
\hline & $\begin{array}{l}\text { with } \mathrm{LTB}_{4} \\
\text { and LPS }\end{array}$ & 720.3818 & 643.9803 & 974.6987 & 468.9765 & 562.63 & 563.1741 \\
\hline
\end{tabular}


Table C2. Relative fold change of I-CAM1 expression in HUVECs in and without the presence of $\mathrm{LTB}_{4}$ and LPS with or without shear exposure.

\begin{tabular}{|c|c|c|c|c|c|c|c|}
\hline \multicolumn{2}{|c|}{ HUVECs condition } & \multicolumn{3}{|c|}{ At $20 \%$ radial location } & \multicolumn{3}{|c|}{ At $85 \%$ radial location } \\
\hline \multirow{4}{*}{$\begin{array}{l}\text { Without } \\
\text { shear } \\
\text { exposure }\end{array}$} & Control & 1 & 1 & 1 & 1 & 1 & 1 \\
\hline & with $\mathrm{LTB}_{4}$ & 0.658019 & 1.685024 & 0.623226 & 0.658019 & 1.685024 & 0.623226 \\
\hline & with LPS & 62.35809 & 57.25539 & 53.99908 & 62.35809 & 57.25539 & 53.99908 \\
\hline & $\begin{array}{l}\text { with } \mathrm{LTB}_{4} \\
\text { and LPS }\end{array}$ & 37.63018 & 25.38928 & 29.62873 & 37.63018 & 25.38928 & 29.62873 \\
\hline \multirow{4}{*}{$\begin{array}{l}\text { Under } \\
\text { shear } \\
\text { exposure } \\
\text { at } 100 \mathrm{rpm} \\
\text { orbital } \\
\text { speed }\end{array}$} & $\begin{array}{c}\text { No } \\
\text { treatment }\end{array}$ & 2.518517 & 3.870795 & 2.122961 & 4.049661148 & 3.577702 & 3.959126 \\
\hline & with $\mathrm{LTB}_{4}$ & 1.758669 & 0.884871 & 2.379852 & 1.016511839 & 1.50771 & 0.450712 \\
\hline & with LPS & 38.32384 & 59.01279 & 48.85959 & 25.93774672 & 25.20518 & 21.68668 \\
\hline & $\begin{array}{c}\text { with } \mathrm{LTB}_{4} \\
\text { and LPS }\end{array}$ & 34.56104 & 39.32541 & 45.51139 & 19.26342205 & 18.93015 & 18.14253 \\
\hline
\end{tabular}

Table C3. Relative fold change of IL-6 expression in HUVECs in and without the presence of $\mathrm{LTB}_{4}$ and LPS with or without shear exposure.

\begin{tabular}{|c|c|c|c|c|c|c|c|}
\hline \multicolumn{2}{|c|}{ HUVECs condition } & \multicolumn{3}{|c|}{ At $20 \%$ radial location } & \multicolumn{3}{|c|}{ At $85 \%$ radial location } \\
\hline \multirow{4}{*}{$\begin{array}{l}\text { Without } \\
\text { shear } \\
\text { exposure }\end{array}$} & Control & 1 & 1 & 1 & 1 & 1 & 1 \\
\hline & with $\mathrm{LTB}_{4}$ & 3.660221 & 1.615337 & 2.959641 & 3.660221 & 1.615337 & 2.959641 \\
\hline & with LPS & 11.84971 & 9.408293 & 20.99083 & 11.84971 & 9.408293 & 20.99083 \\
\hline & $\begin{array}{l}\text { with } \mathrm{LTB}_{4} \\
\text { and LPS }\end{array}$ & 15.67928 & 13.44231 & 19.09798 & 15.67928 & 13.44231 & 19.09798 \\
\hline \multirow{4}{*}{$\begin{array}{c}\text { Under } \\
\text { shear } \\
\text { exposure } \\
\text { at } 100 \mathrm{rpm} \\
\text { orbital } \\
\text { speed }\end{array}$} & $\begin{array}{c}\text { No } \\
\text { treatment }\end{array}$ & 6.185467 & 3.004064 & 3.94433 & 1.787345834 & 3.117498 & 0.342108 \\
\hline & with $\mathrm{LTB}_{4}$ & 4.045904 & 7.560395 & 6.42365 & 3.719439423 & 3.090653 & 1.103295 \\
\hline & with LPS & 45.24271 & 34.78652 & 33.98032 & 26.84572481 & 22.05327 & 16.38023 \\
\hline & $\begin{array}{c}\text { with } \mathrm{LTB}_{4} \\
\text { and LPS }\end{array}$ & 59.09772 & 47.37037 & 53.68796 & 16.19965606 & 17.04474 & 10.66216 \\
\hline
\end{tabular}




\section{APPENDIX - D}

Table D1. System properties and related dimensionless parameters for Figure 2.10

\begin{tabular}{|c|c|c|c|c|c|c|c|c|c|}
\hline $\begin{array}{l}\text { Orbital } \\
\text { speed }\end{array}$ & Gravity & $\begin{array}{c}\text { Radius } \\
\text { of } \\
\text { orbit }\end{array}$ & $\begin{array}{l}\text { Radius } \\
\text { of dish }\end{array}$ & $\begin{array}{l}\text { Mean } \\
\text { height } \\
\text { of } \\
\text { fluid } \\
\mathrm{cm}\end{array}$ & viscosity & $\begin{array}{l}\text { Slope } \\
\text { ratio }\end{array}$ & $\begin{array}{c}\text { Froude } \\
\text { No. }\end{array}$ & $\begin{array}{c}\text { Stokes } \\
\text { No. }\end{array}$ & $\begin{array}{c}\text { Reynolds } \\
\text { No. }\end{array}$ \\
\hline 60 & 9.8 & 0.95 & 1.75 & 0.2 & $1.0100 \mathrm{E}-03$ & 0.335 & 0.785 & 4.982 & 218 \\
\hline 90 & 9.8 & 0.95 & 1.75 & 0.2 & $1.0100 \mathrm{E}-03$ & 0.753 & 1.178 & 6.102 & 326 \\
\hline 120 & 9.8 & 0.95 & 1.75 & 0.2 & $1.0100 \mathrm{E}-03$ & 1.338 & 1.570 & 7.056 & 435 \\
\hline 150 & 9.8 & 0.95 & 1.75 & 0.2 & $1.0100 \mathrm{E}-03$ & 2.091 & 1.963 & 7.877 & 544 \\
\hline 180 & 9.8 & 0.95 & 1.75 & 0.2 & $1.0100 \mathrm{E}-03$ & 3.010 & 2.355 & 8.629 & 653 \\
\hline 210 & 9.8 & 0.95 & 1.75 & 0.2 & $1.0100 \mathrm{E}-03$ & 4.098 & 2.747 & 9.321 & 762 \\
\hline
\end{tabular}

Table D2. System properties and related dimensionless parameters corresponding to DOSI analysis cases in Chapter Four.

\begin{tabular}{|c|c|c|c|c|c|c|c|c|c|c|}
\hline $\begin{array}{l}\text { Orbital } \\
\text { speed }\end{array}$ & Cases & Gravity & $\begin{array}{c}\text { Radius } \\
\text { of } \\
\text { orbit } \\
\text { cm }\end{array}$ & $\begin{array}{l}\text { Radius } \\
\text { of dish }\end{array}$ & $\begin{array}{l}\text { Mean } \\
\text { height } \\
\text { of } \\
\text { fluid } \\
\mathrm{cm}\end{array}$ & viscosity & $\begin{array}{c}\text { Slope } \\
\text { ratio }\end{array}$ & $\begin{array}{c}\text { Froude } \\
\text { No. }\end{array}$ & $\begin{array}{c}\text { Stokes } \\
\text { No. }\end{array}$ & $\begin{array}{c}\text { Reynolds } \\
\text { No. }\end{array}$ \\
\hline 50 & 1 & 9.8 & 0.95 & 2.5 & 0.2 & 0.00072 & 0.332 & 0.934 & 5.387 & 363 \\
\hline 75 & 2 & 9.8 & 0.95 & 2.5 & 0.2 & 0.00072 & 0.747 & 1.402 & 6.597 & 544 \\
\hline 100 & 3 & 9.8 & 0.95 & 2.5 & 0.2 & 0.00072 & 1.327 & 1.869 & 7.618 & 725 \\
\hline 150 & 4 & 9.8 & 0.95 & 2.5 & 0.2 & 0.00072 & 2.987 & 2.803 & 9.330 & 1090 \\
\hline 200 & 5 & 9.8 & 0.95 & 2.5 & 0.2 & 0.00072 & 5.309 & 3.738 & 10.773 & 1450 \\
\hline
\end{tabular}




\section{APPENDIX - E}

\section{NOMENCLATURE}

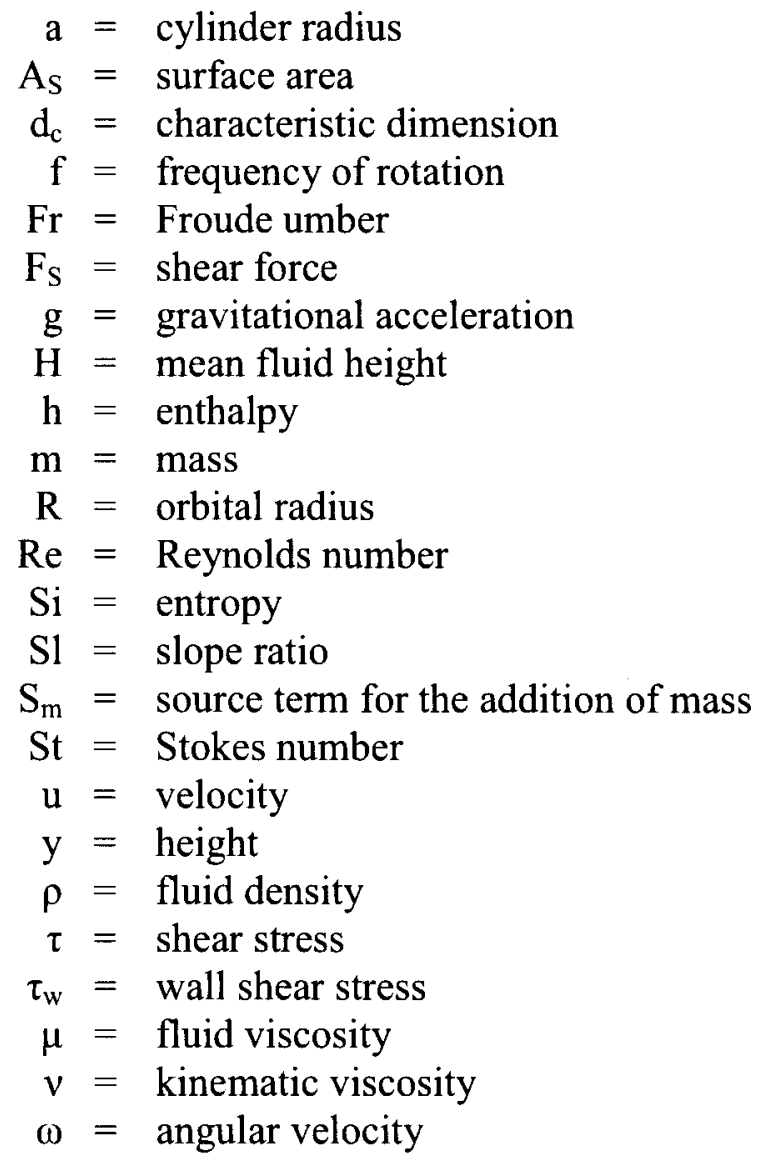


CURRICULUM VITAE

NAME:

ADDRESS:

DOB:

EDUCATION:

New Mexico State University, Las Cruces, NM, USA

2006-2011
M.S., Chemical Engineering

$2004-2006$

Ph.D., Chemical Engineering

University of Louisville, Louisville, KY, USA

\section{AMLAN CHAKRABORTY}

J.B. Speed School of Engineering

Chemical Engineering Department

University of Louisville, Louisville, KY, USA

West Bengal, India - November, 1976

B. S., Chemical Engineering

Haldia Institute of Technology, West Bengal, India $1996-2000$ 


\section{AWARDS}

- Outstanding Doctoral Dissertation Completion Award - University of Louisville, January, 2011.

- Student Travel Award from Graduate Student Council (GSC) for attending Summer Bioengineering Conference and presenting a paper in Naples, Florida, June 16-19, 2010.

- Teaching Assistant, University of Louisville

Aug 2008 - Aug 2010

- Engineering Graduate Fellowship, University of Louisville

Aug 2006 - July 2008

- Best Poster Presentation Award at 2006 New Mexico State University Research Council Fair, NM

- Research Assistant, New Mexico State University Jan 2004 - July 2006

\section{AFFILIATIONS}

- American Institute of Chemical Engineers (AICHE)

\section{PUBLICATIONS}

- A. Chakraborty, J. Thomas, M. K. Sharp, R.E. Berson. Fluid Dynamic Analysis Of Oscillating Flow In An Orbiting Dish And Effects Of Dimensionless Parameters (Slope Ratio, Stokes Number And Froude Number) On Fluid Flow”. Manuscript on process. 
- Amlan Chakraborty, Venkatakrishna Jala, M. Keith Sharp, R. Eric. Berson, Haribabu Bodduluri. "Bi-directional Oscillatory Fluidic Shear Stress Increases Pro-Atherogenic Gene Expressions (I-CAMI, E-Selectin, IL-6) in Endothelial”. To be submitted.

- Amlan Chakraborty, Sutirtha Chakraborty, Venkatakrishna Jala, Haribabu Bodduluri, M. Keith Sharp, R.Eric. Berson. "Effects of Bidirectional Oscillatory Shear Stress on Endothelial Cell Proliferation and Morphology". In revision, Biotechnology \& Bioengineering, 2010.

- J. Thomas; A. Chakraborty; M. K. Sharp, R.E. Berson. "Comparison of Computational, Analytical And Experimental Resolution Of Wall Shear Stress In An Orbiting Petri-Dish", Biotechnology Progress, Accepted - Aug 2010.

\section{SELECTED NATIONAL MEETING PRESENTATIONS}

- Amlan Chakraborty, M. Keith Sharp, Venkatakrishna Jala, Haribabu Bodduluri, R. Eric Berson, "Directional Oscillatory Shear Index in Orbital Petri-dishes", AICHE 2010, Salt Lake City, UT, Nov 2010.

- Amlan Chakraborty, Venkatakrishna Jala, Haribabu Bodduluri, M. Keith Sharp, R. Eric Berson. "Effects of Directional Oscillatory Shear Index on Endothelial Cell Proliferation and Morphology". Summer Bioengineering Conference, Naples, Florida, June 2010

- Amlan Chakraborty, Venkatakrishna Jala, Haribabu Bodduluri, R. Eric Berson, “Effects Of Oscillatory Fluid Shear Stress On Endothelial Cellular Responses In The Presence Of LTB4 And LPS", AICHE 2009, Nashville, TN, Nov 2009. 
- Amlan Chakraborty, Venkatakrishna Jala, Haribabu Bodduluri, R Eric Berson. "In Vitro Study Of The Effects Of Oscillating Fluidic Shear Stress On Endothelial Cellular Responses Including Cell Proliferation And Atherogenic Gene Expression At Varying Locations Of The Dish." Research Louisville, Louisville, KY, Oct 2009.

- Amlan Chakraborty, M. Keith Sharp and R. Eric Berson, "Complete Spatial and Temporal Resolution of Shear on Cells Grown In Rotary Orbital Shakers", AICHE 2007, Salt Lake City, UTAH, Nov 2007

- Amlan Chakraborty, R Eric Berson. "Measurement Of Wall Shear Stress Of Liquid In Orbital Cylindrical Dish By Computational Fluid Dynamics: Comparison of Generated Shear by Simulation and Previous Experimentation" Research Louisville - Oct - 2007

- Amlan Chakraborty, S.H. Valluri, S. Deng. "Direct Contact Membrane Distillation For Brackish Water Desalination: Comparison of Flat Sheet Membrane and Hollow Fiber Membrane Modules", New Mexico Water Research Symposium 2006, Soccoro, NM, Aug 2006 